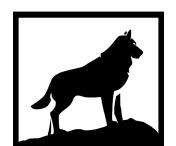

Michigan Technological

1 в 8 5 University
Michigan Technological University Digital Commons @ Michigan Tech

RISK-BASED ASSESSMENT AND STRENGTHENING OF ELECTRIC POWER SYSTEMS SUBJECTED TO NATURAL HAZARDS

Abdullahi Salman

Michigan Technological University, amsalman@mtu.edu

Copyright 2016 Abdullahi Salman

Recommended Citation

Salman, Abdullahi, "RISK-BASED ASSESSMENT AND STRENGTHENING OF ELECTRIC POWER SYSTEMS SUBJECTED TO NATURAL HAZARDS", Open Access Dissertation, Michigan Technological University, 2016.

https://doi.org/10.37099/mtu.dc.etdr/207

Follow this and additional works at: https://digitalcommons.mtu.edu/etdr

Part of the Civil Engineering Commons, and the Structural Engineering Commons 


\section{RISK-BASED ASSESSMENT AND STRENGTHENING OF ELECTRIC POWER SYSTEMS SUBJECTED TO NATURAL HAZARDS}

By

Abdullahi M. Salman

\section{A DISSERTATION}

Submitted in partial fulfillment of the requirements for the degree of DOCTOR OF PHILOSOPHY

In Civil Engineering

MICHIGAN TECHNOLOGICAL UNIVERSITY

2016

(C) 2016 Abdullahi M. Salman 
This dissertation has been approved in partial fulfillment of the requirements for the Degree of DOCTOR OF PHILOSOPHY in Civil Engineering.

Department of Civil and Environmental Engineering

Dissertation Advisor: $\quad$ Dr. Yue $\mathrm{Li}$

Committee Member: $\quad$ Dr. William M. Bulleit

Committee Member: $\quad$ Dr. Raymond A. Swartz

Committee Member: Dr. Chee-Wooi Ten

Department Chair: $\quad$ Dr. David Hand 
Ta Engr. Laval A. Audi. 


\section{Table of Contents}

List of Tables ............................................................................................................................. viii

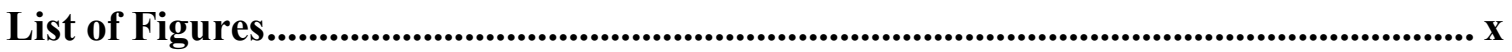

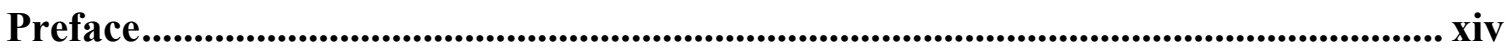

Acknowledgement ............................................................................................................... $\mathrm{xV}$

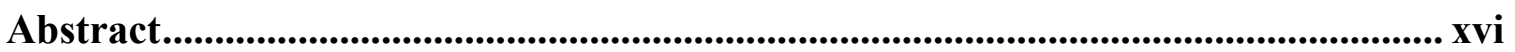

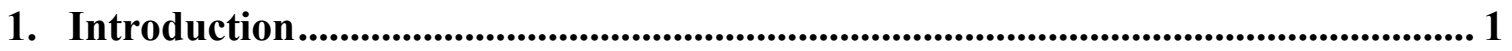

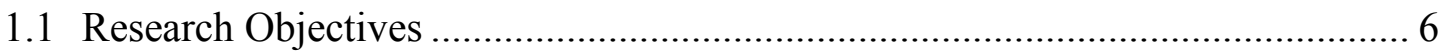

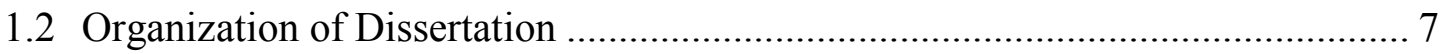

2. Background ................................................................................................................... 10

2.1 Electric Power Systems ……………………………….................................... 10

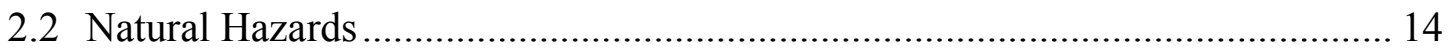

2.3 Infrastructure Risk Assessment and Management …………………………..... 20

3. System Reliability Model.................................................................................................... 23

3.1 Review of Existing Topological-Based Methods................................................. 24

3.2 Accessibility of System Components ................................................................. 26

3.3 Topological-Based System Reliability Formulation ............................................. 34

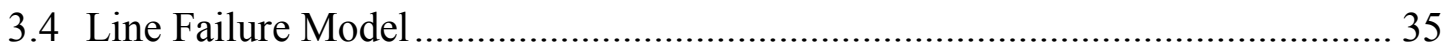

4. Targeted Hardening of Distribution Systems Subjected to Hurricanes ................ 39

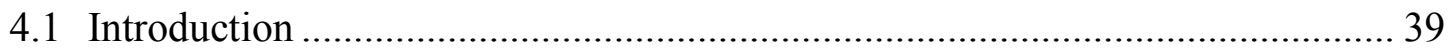

4.2 Power Distribution System Model ................................................................... 42

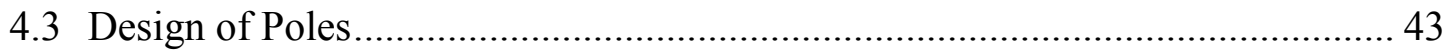

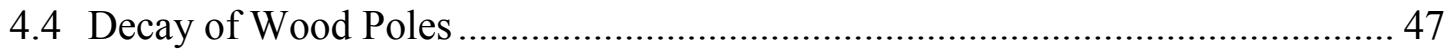

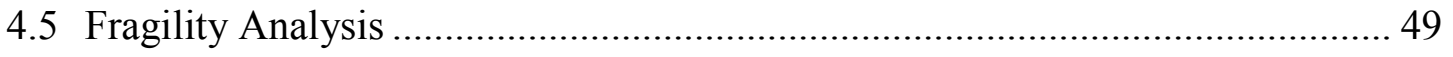


4.6 Hurricane Risk Assessment ..................................................................... 53

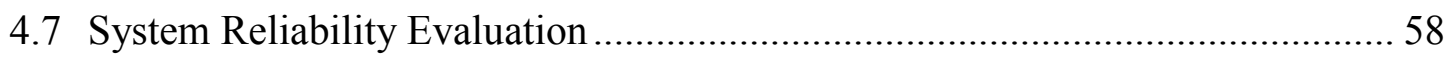

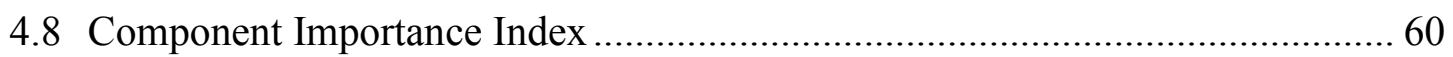

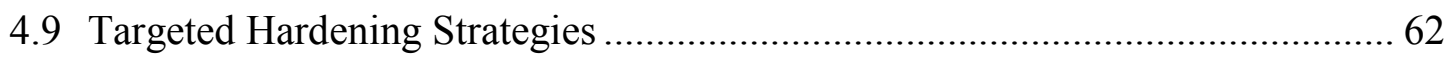

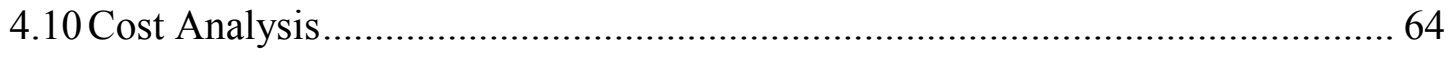

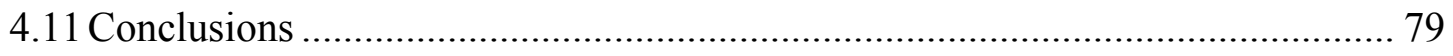

5. Potential Impact of Climate Change on Distribution Systems Subjected to

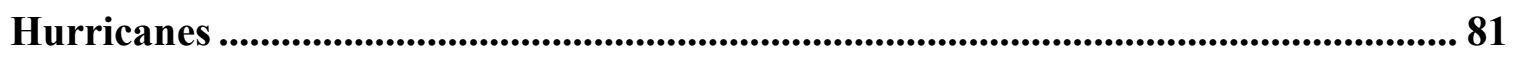

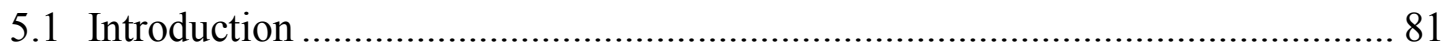

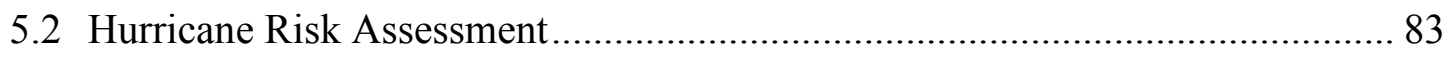

5.3 Non-stationary Hurricane Wind Model due to Climate Change ........................ 96

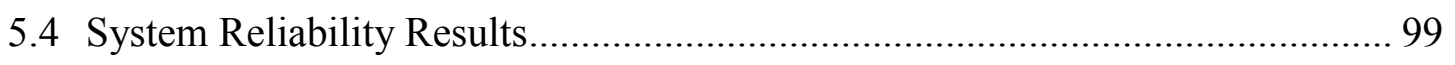

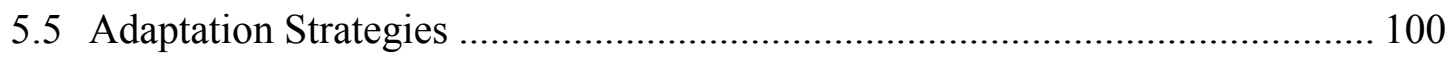

5.6 Life Cycle Cost Analysis Result ............................................................ 102

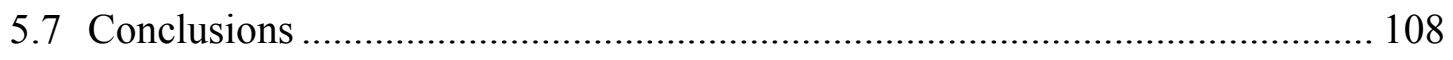

6. Reconfigurability Enhancement for Distribution Systems Subjected to

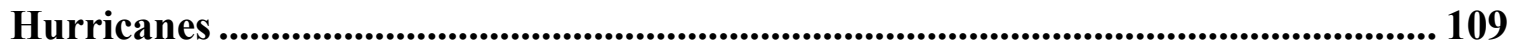

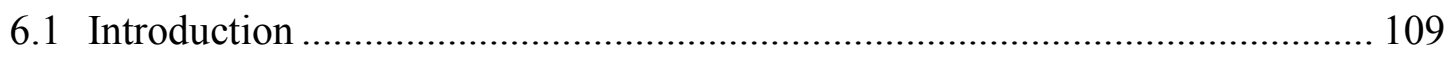

6.2 Power Distribution System Model ......................................................... 111

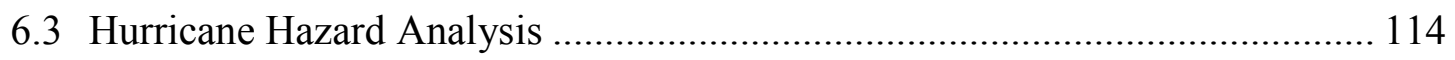

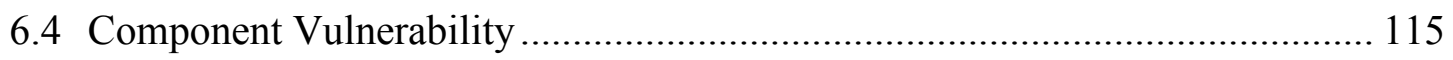

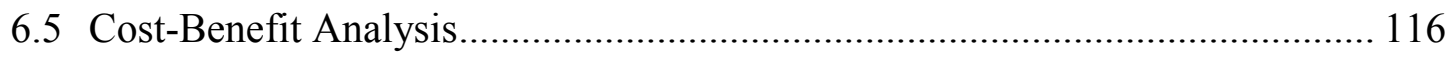

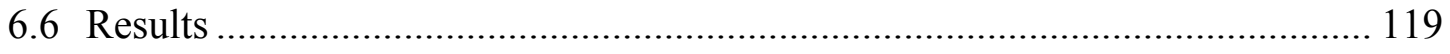

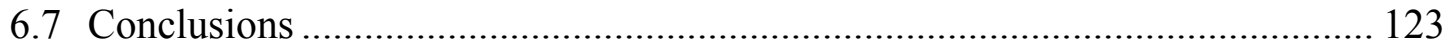

7. Multi-Hazard Risk Assessment Framework ................................................. 125 


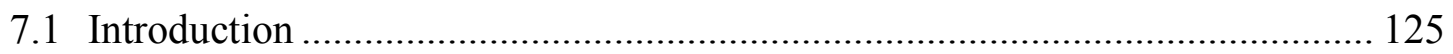

7.2 Electric Power System.................................................................................. 129

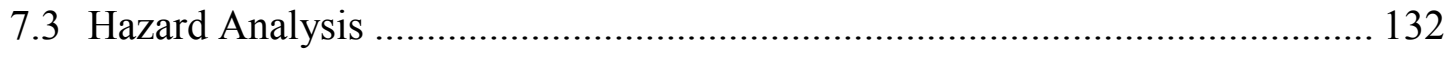

7.4 Component Vulnerability Analysis ............................................................... 137

7.5 Component Risk Assessment ......................................................................... 141

7.6 System Reliability Results.......................................................................... 145

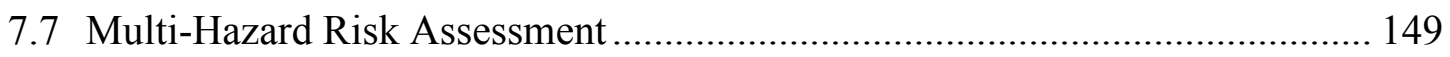

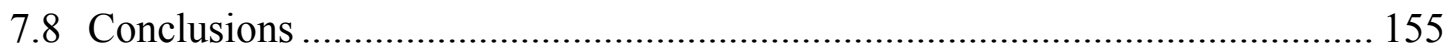

8. Multi-Hazard Risk Mitigation Framework............................................................. 156

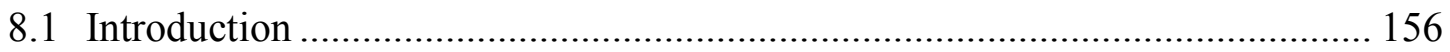

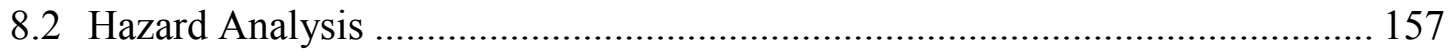

8.3 Component Vulnerability ………………………………………………..... 165

8.4 Multi-Hazard Risk Assessment .................................................................... 166

8.5 Component Importance Measure ……………………................................... 172

8.6 Multi-Hazard Mitigation Strategies.................................................................. 177

8.7 Evaluation of Cost-Effectiveness through Life-cycle Cost Analysis ................. 180

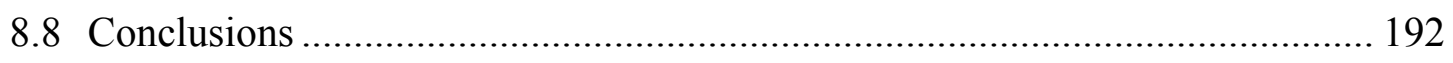

9. Summary, Conclusions, Applications, and Future Work ........................................ 194

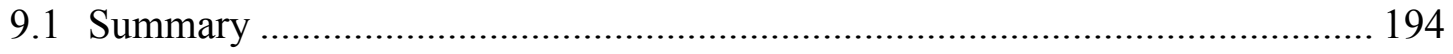

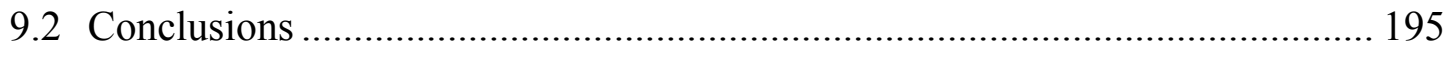

9.3 Applications and Recommendations for Future Study.................................... 197

10. References..................................................................................................................... 200

Appendix A: Document showing that Fig. 2.2 is in the public domain..................... 216

Appendix B: Permission to publish Chapter 4.............................................................. 220

Appendix C: Permission to use Fig. 4.2 \& 4.3 .................................................................... 227 
Appendix D: Permission to publish Chapter 5................................................................... 229

Appendix E: Permission to use base map of Figure 6.2 .......................................... 231 


\section{List of Tables}

Table 2.1 General information on the U.S. electric grid.......................................... 14

Table 2.2 Summary of causes of major power outages in the U.S............................ 15

Table 2.3: Saffir-Simpson hurricane scale and summary of hurricanes that hit the U.S.. 16

Table 2.4 Summary of damage to power systems caused by hurricanes ........................ 17

Table 2.5 Summary of damage to power systems caused by earthquakes ..................... 20

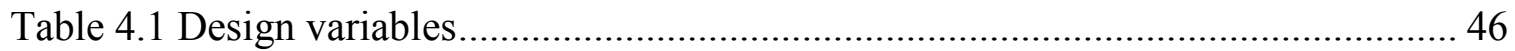

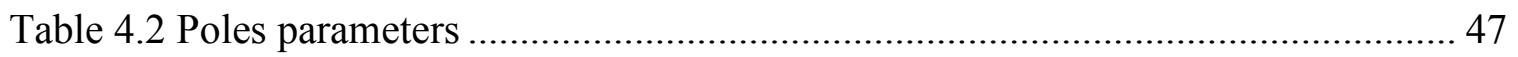

Table 4.3 Strength and load parameters and their statistics...................................... 51

Table 4.4 Load profiles for consumers across Micropolis............................................59

Table 4.5 System reliability comparison for scenario-based analysis ........................... 63

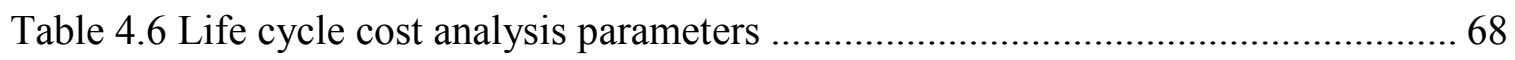

Table 4.7 LCCA results for probabilistic hurricane analysis - Case $1(\$ 1,000 \mathrm{~s}) \ldots \ldots \ldots \ldots . . . .70$

Table 4.8 LCCA results with reductions in revenue and economic losses $(\$ 1,000 \mathrm{~s}) \ldots \ldots .71$

Table 4.9 LCCA results for probabilistic hurricane analysis - Case 2 $(\$ 1,000 \mathrm{~s}) \ldots \ldots \ldots \ldots . . . .72$

Table 4.10 LCCA results with reductions in revenue and economic losses $(\$ 1,000 \mathrm{~s}) \ldots . .72$

Table 4.11 Cost analysis results for scenario hurricane - Case $1(\$ 1,000$ s) ................... 74

Table 4.12 Cost analysis results with reductions in revenue and economic losses $(\$ 1,000 \mathrm{~s})$

Table 4.13 Cost analysis results for scenario hurricane - Case $2(\$ 1,000$ s) ................... 77

Table 4.14 Cost analysis results with reductions in revenue and economic losses $(\$ 1,000 \mathrm{~s})$

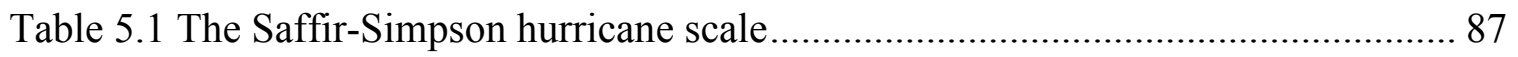


Table 5.2 Hurricane occurrence in different parts of Florida from $1851-2014$.

Table 5.3 Statistics of hurricane simulation parameters for different regions of Florida. 91

Table 5.4 Comparison of wind speeds corresponding to different return periods 95

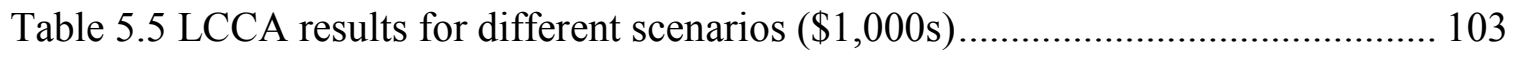

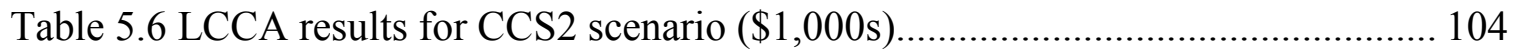

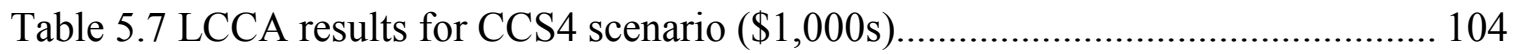

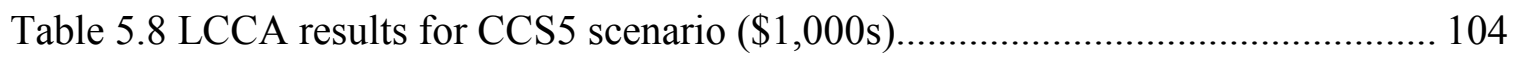

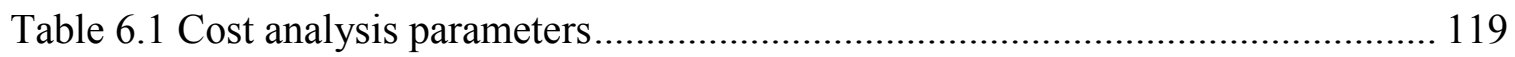

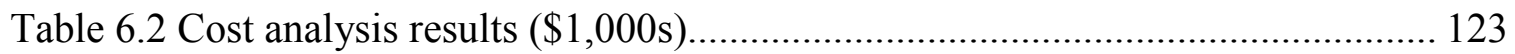

Table 7.1 Notional power system information ...................................................... 132

Table 7.2 Lognormal parameters for seismic fragility of substations with anchored

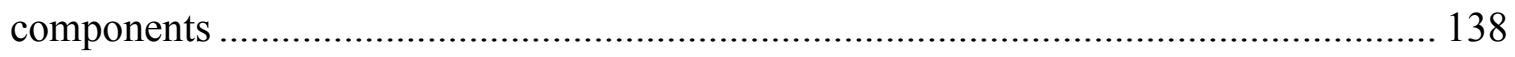

Table 7.3 Electric power system reliability results for Charleston ............................. 148

Table 7.4 Multi-hazard risk comparison based on stipulated design hazard level ......... 153

Table 7.5 Multi-hazard risk comparison based on annual probability of damage of components 154

Table 8.1 Selected scenario earthquakes and their annual probabilities of occurrence.. 160

Table 8.2 Statistics of hurricane simulation parameters ......................................... 164

Table 8.3 Lognormal parameters for seismic fragility of substations with standard

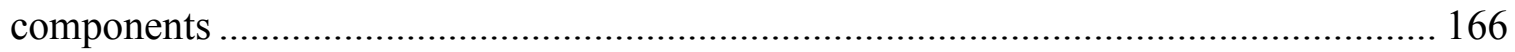

Table 8.4 Lognormal parameters for seismic fragility of substations with anchored

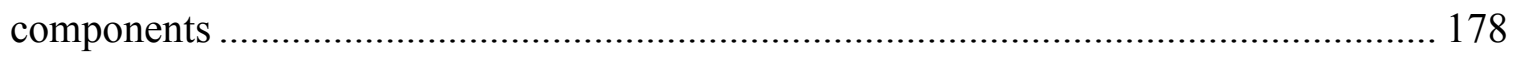

Table 8.5 Life cycle cost analysis parameters .................................................... 186

Table 8.6 Cost analysis results for Charleston, SC (\$1000s) .................................... 188

Table 8.7 Cost analysis results for New York, NY (\$1000s) ................................... 188 


\section{List of Figures}

Figure 2.1 Schematic of an electric power system ……….......................................... 12

Figure 2.2 U.S. seismic hazard map (PGA, 2\% in 50 years) ........................................ 18

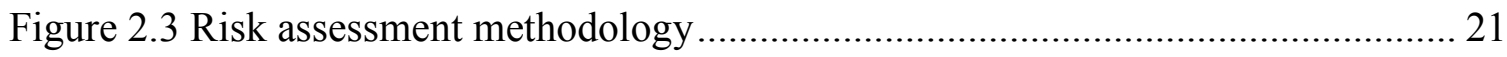

Figure 2.4 Risk management framework ............................................................... 22

Figure 3.1 Topology of electric power sub-systems .................................................... 26

Figure 3.2 Schematic of a radial distribution system..................................................... 28

Figure 3.3 Reliability block diagram for power delivery to line 7 ................................ 28

Figure 3.4 Simple schematic of distribution system with redundancy ........................... 29

Figure 3.5 Fault tree diagram for failure of power delivery to Line 4 ............................. 29

Figure 3.6 Schematic of a networked power system ………........................................... 30

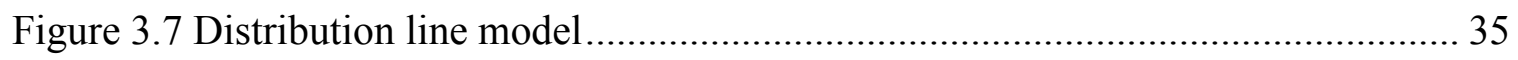

Figure 4.1 Flowchart of proposed framework ………………........................................ 41

Figure 4.2 Micropolis power distribution system ......................................................... 43

Figure 4.3 Micropolis power distribution system line diagram ....................................... 43

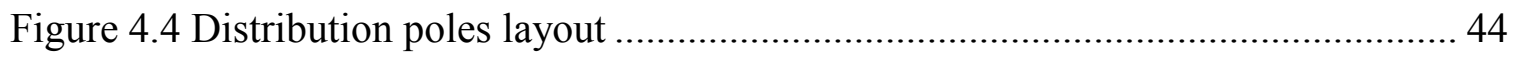

Figure 4.5 Residual strength of poles as a function of time............................................. 48

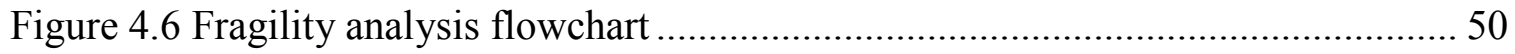

Figure 4.7 Fragility curves of three-phase line poles at $0,20,40$, and 60 years............... 52

Figure 4.8 Fragility curves of single-phase line poles at $0,20,40$, and 60 years ............ 52

Figure 4.9 Annual probability of failure with age …………………………………..... 55

Figure 4.10 Surface wind speed variation at the center of study area .............................. 57

Figure 4.11 Reliability of line 33 with age using probabilistic hurricane analysis .......... 58 
Figure 4.12 System reliability with age using probabilistic hurricane analysis 60

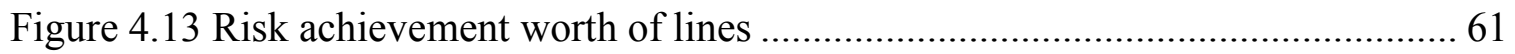

Figure 4.14 System reliability improvement after targeted hardening .......................... 63

Figure 4.15 Impact of repair time on net benefit of mitigation strategies (Case 1) ......... 75

Figure 4.16 Impact of number of repair crew units on net benefit (Case 1)................... 76

Figure 4.17 Impact of repair time on net benefit of mitigation strategies (Case 2)......... 78

Figure 4.18 Impact of number of repair crew units on net benefit (Case 2)................... 79

Figure 5.1 Hurricane simulation model flowchart.................................................. 90

Figure 5.2 Surface wind speed variation for a simulated hurricane ........................... 92

Figure 5.3 CDF of max annual hurricane wind speed with fitted distributions............... 94

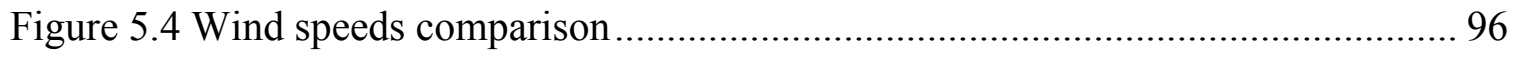

Figure 5.5 Hazard curves for different climate scenarios ........................................ 99

Figure 5.6 System reliability results for different climate scenarios ......................... 100

Figure 5.7 System reliability improvement for CCS5 ........................................ 102

Figure 5.8 LCC results for different climate scenarios.......................................... 103

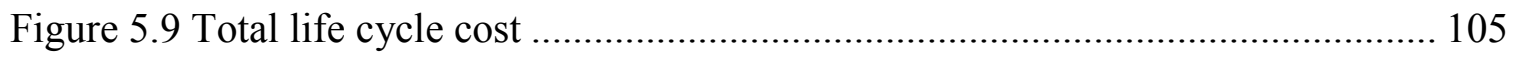

Figure 5.10 Net benefit of adaptation strategies .................................................. 106

Figure 5.11 Net benefit considering reduction in revenue and economic losses........... 107

Figure 6.1 Schematic of proposed storm hardening strategy.................................. 110

Figure 6.2 Notional power distribution system of parts of Florida (3-phase feeder lines)

Figure 6.3 Vortex shape of gradient wind speed for the 3 historical hurricanes at landfall 115

Figure 6.4 Fragility curve of main feeder line poles 116 
Figure 6.5 System reliability results for Hurricane Jeanne........................................ 121

Figure 6.6 System reliability results for Hurricane Wilma...................................... 121

Figure 6.7 System reliability results for Hurricane Charley ..................................... 122

Figure 7.1 Flowchart of proposed multi-hazard risk assessment framework ............... 128

Figure 7.2 Notional electric power system ......................................................... 130

Figure 7.3 Seismic hazard curve for Charleston, SC (32.8oN 79.9oW)..................... 135

Figure 7.4 Probabilistic seismic hazard analysis .................................................. 135

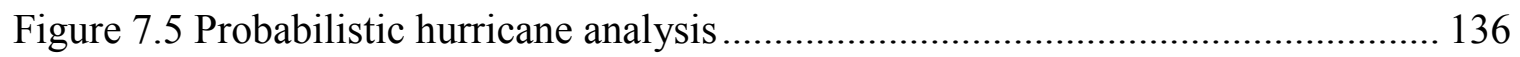

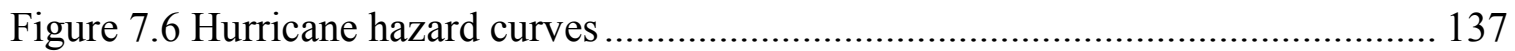

Figure 7.7 Seismic fragility curves of substations for extensive damage state ............. 139

Figure 7.8 Fragility curve of transmission support structures ................................. 141

Figure 7.9 Component risk results for Charleston .............................................. 144

Figure 7.10 Accessibility of demand substations in Charleston ............................... 147

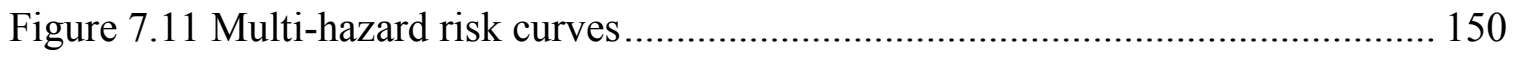

Figure 7.12 Annual probability of system failure .................................................. 154

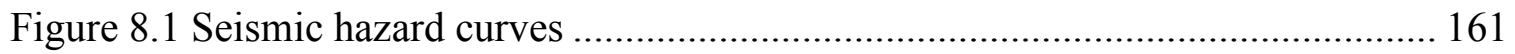

Figure 8.2 Hurricane simulation model flow chart .............................................. 163

Figure 8.3 Hurricane hazard curves .................................................................. 164

Figure 8.4 Multi-hazard risk curves ................................................................... 167

Figure 8.5 Comparison between probabilistically weighted deterministic scenarios method

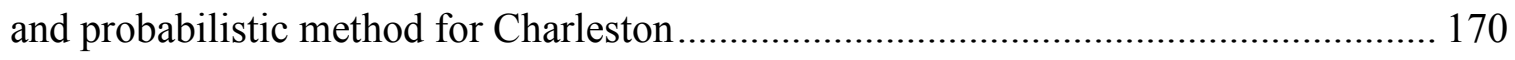

Figure 8.6 Comparison between probabilistically weighted deterministic scenarios method and probabilistic method for New York .......................................................... 171

Figure 8.7 Risk achievement worth of components in Charleston ............................. 173 
Figure 8.8 Importance index of components in Charleston ..................................... 176

Figure 8.9 Fragilities curves of existing and hardened transmission structures ............ 179

Figure 8.10 Net benefit of mitigation strategies (Charleston, SC) ............................. 189

Figure 8.11 Net benefit of mitigation strategies (New York, NY) ............................. 189

Figure 8.12 Net benefit of mitigation strategies with $2 \%$ discount rate (Charleston, SC) 191

Figure 8.13 Net benefit of mitigation strategies with 2\% discount rate (New York, NY) 192 


\section{Preface}

A version of Chapter 4 has been published as Evaluating System Reliability and Targeted Hardening Strategies of Power Distribution Systems Subjected to Hurricanes in Reliability Engineering \& System Safety (144 (2015), pp 319-333). The author developed the framework and relevant equations as well as performed the case study and analysis. Dr. Yue Li and Dr. Mark Stewart reviewed the paper and offered valuable suggestions.

A version of Chapter 5 has been published as Assessing Climate Change Impact on system reliability of Power Distribution Systems Subjected to Hurricanes in ASCE Journal of Infrastructure Systems (10.1061/(ASCE)IS.1943-555X.0000316). The author developed the framework and relevant equations as well as performed the case study and analysis. Dr. Yue Li reviewed the paper and offered valuable suggestions.

A version of Chapter 6 has been submitted to ASCE Journal of Infrastructure Systems with the title: Reconfigurability Enhancement for Power Distribution Systems under Hurricane Hazard. The author developed the framework and relevant equations as well as performed the case study and analysis. Dr. Chee-Wooi Ten and Dr. Yue Li reviewed the paper and offered valuable suggestions.

A version of Chapter 7 has been submitted to ASCE Journal of Structural Engineering with the title: Multi-hazard Risk Assessment of Electric Power Systems. The author developed the framework and relevant equations as well as performed the case study and analysis. Dr. Yue Li reviewed the paper and offered valuable suggestions.

A version of Chapter 8 has been submitted to Reliability Engineering \& System Safety with the title: A probabilistic Framework for Multi-Hazard Mitigation Strategies for Electric Power systems. The author developed the framework and relevant equations as well as performed the case study and analysis. Dr. Yue Li reviewed the paper and offered valuable suggestions. 


\section{Acknowledgement}

This dissertation would not have been possible without the guidance and help of several individuals who in one way or another contributed and extended their valuable assistance in the preparation and completion of this study. First of all, I would like to express my heartfelt gratitude to my supervisor and mentor, Dr. Yue Li for his guidance, advice, encouragement, and friendship during the course of my stay at Michigan Tech.

I am also grateful to Dr. William Bulleit and Dr. Andrew Swartz for the awesome classes, their valuable time serving as committee members, and overall support during my stay at Michigan Tech. Their passion for teaching is something I aspire to emulate. I will also like to thank Dr. Chee-Wooi Ten for all the time he spent teaching me about electric power systems, providing valuable resources to help with my research, and providing guidance and advice on graduate research and life in general.

I owe my deepest gratitude to my parents: Engr. Lawal A. Audi, Dr. Amina Odidi, Dr. M.D. Audi, Hajiya Hadiza Audi, and Safiya Mukhtar. Their love, support, and guidance made me the person I am today. Finally, I will like to thank my siblings, cousins, uncles, aunties, and all my relatives for their unwavering love, encouragement, and an awesome life. 


\begin{abstract}
Modern economic and social activities are dependent on a complex network of infrastructure systems that are highly interdependent. Electric power systems form the backbone of such complex network as most civil infrastructure systems cannot function properly without reliable power supply. Electric power systems are vulnerable to extensive damage due to natural hazards, as evident in recent hazard events. Hurricanes, earthquakes, floods, tornados and other natural hazards have caused billions of dollars in direct losses due to damage to power systems and indirect losses due to power outages, as well as social disruption. There is, therefore, a need for a comprehensive framework to assess and mitigate the risk posed by natural hazards to electric power systems. Electric power systems rely on various components that work together to deliver power from generating units to customers. Consequently, any reliable risk assessment methodology needs to take into account how the different components interact. This requires a system-level risk assessment approach. This research presents a framework for system-level risk assessment and management for electric power systems subjected to natural hazards. Specifically, risk due to hurricanes and earthquakes, as well as the combined effect of both is considered. The framework incorporates a topological-based system reliability model, probabilistic and scenario-based hazard analysis, climate change modeling, component vulnerability, component importance measure, multi-hazard risk assessment, and cost analysis. Several risk mitigation strategies are proposed; their efficiency and cost-effectiveness are studied. The developed framework is intended to assist utility companies and other stakeholders in making a risk-informed decision regarding short- and long-term investment in natural hazard risk mitigation for electric power systems. The framework can be used to identify certain parts of the system to strengthen, compare the efficiency and cost-effectiveness of various risk mitigation strategies using life-cycle cost analysis, compare risks posed by different natural hazards, and prioritize investment in the face of limited resources.
\end{abstract}




\section{Introduction}

Civil infrastructure systems such as electric power systems, water distribution systems, transportation networks, gas supply systems, etc. are the backbone of modern economies and essential for public wellbeing. For safety, security, prosperity, and social welfare, such critical infrastructure systems need to be reliable, robust, and resilient. Most civil infrastructure systems cannot function properly without reliable power supply. Emergency response units, telecommunication networks, traffic control systems, healthcare facilities, etc., all depend on the electric power system to function. As such, the electric power system is among the most critical of all the civil infrastructure systems. Thus, the loss of electricity can cause billions of dollars in direct and indirect economic losses.

Electric power systems are subjected to numerous disturbances ranging from small disturbances caused by common cause failures to major disturbances caused by natural hazards. Natural hazards that threaten power systems include hurricanes, earthquakes, floods, severe thunderstorms, and tornadoes. In the event of natural disasters, continuous supply of electricity is essential not only to critical buildings such as hospitals and fire stations but to the public as a whole. Hurricanes and earthquakes are among the most devastating natural hazards that can cause extensive damage and prolonged power outages (Stewart, 2004; Pasch et al., 2005; Blake et al., 2013; Romero et al., 2015; Noda, 2001b).

The extensive damage potential of hurricanes on power systems has been well documented historically. From the four major hurricanes that hit Florida in 2004 to the 2008 hurricane season in Texas, the cost of hurricane damage to power systems is in the billions (USDOE, 2005; Hoffman et al., 2009). In 2004, four major hurricanes struck Florida causing a combined economic loss of over $\$ 20$ billion and damaging every segment of Florida's electricity infrastructure which resulted in a power outage to over 9.6 million customers combined (USDOE, 2005). In 2005, hurricanes Katrina, Rita, and Wilma struck the U.S. causing extensive damage to power systems across several states (Knabb et al., 2005; NOAA, 2005; Pasch et al., 2006). Recently in 2012, hurricane Sandy caused severe damage 
to the power system of several coastal states causing over 8.5 million customers to lose power for weeks and even months in some areas (Blake et al., 2013). When it comes to damage due to hurricanes, the distribution part of the power system is the most vulnerable (Davidson et al., 2003). For example, between 1998 and 2009, electric utility companies in Texas incurred about $\$ 1.8$ billion in restoration costs due to hurricanes with $80 \%$ of the costs attributed to the distribution system (Brown, 2009).

Investment in the power sector is considered a long-term investment due to the service life of the assets, which can be as long as 100 years (Mendiluce, 2014). It is, therefore, imperative for utility companies to consider the uncertainties inherent in such long-term investments that will impact return on investment as well as customer satisfaction. One such uncertainty is the potential impact of climate change on hurricane hazard. In a National Climate Assessment report which summarizes the impacts of climate change on the United States, it is stated that "the intensity, frequency, and duration of North Atlantic hurricanes, as well as the frequency of the strongest (Category 4 and 5) hurricanes, have all increased since the early 1980s... Hurricane-associated storm intensity and rainfall rates are projected to increase as the climate continues to warm" (Walsh et al., 2014). With the potential increase in the intensity of hurricanes in the long-term due to climate change, the cost of damage to power systems is expected to rise.

Electric power systems are also vulnerable to damage due to earthquakes. For example, the 1994 Northridge earthquake caused over 2.5 million customers to lose power and resulted in direct losses of about $\$ 138$ million to Los Angeles Department of Water and Power (Dong et al., 2004; Schiff et al., 1995). Similarly, the 1995 Great Hanshin earthquake, 2008 Wenchuan earthquake, as well as the 2010 Chile earthquake caused various levels of damage to electric power systems (Noda, 2001a; Eidinger, 2009; Romero et al., 2015). When it comes to damage due to earthquakes, brittle substation components with considerable weight are the most vulnerable (Vanzi, 1996; Eidinger \& Kempner, 2012). 
The extent of damage to electric power systems due to natural hazards depends not only on the frequency and intensity of such hazards but also on the number of different types of hazards that the systems are exposed to. Most parts of the world are vulnerable to multiple hazards that can be concurrent/non-concurrent and dependent/independent (Li et al., 2012). Over the lifespan of electric power systems located in regions affected by more than one hazard, such systems can be affected by multiple hazards that differ in nature and can occur simultaneously or otherwise. For example, hurricanes and earthquakes have a very low probability of occurring simultaneously and are considered independent non-concurrent hazards. However, the life cycle cost of systems located in areas vulnerable to both hurricanes and earthquakes depends on the combined effect of both hazards. Therefore, long-term risk mitigation strategies need to consider the effect of multiple independent hazards as certain mitigation strategies for one hazard might be ineffective or even increase the risk for other hazards (Bell \& Glade, 2004).

Aging infrastructure has also been determined to be one of the main issues facing the power system (ASCE, 2013). Aging of components increases the vulnerability of the system in cases of natural disasters such as hurricanes. Wood distribution poles, for example, are susceptible to decay as they age which causes a reduction in strength. This is of particular concern as most of the distribution poles in the U.S. are wood poles (Gustavsen \& Rolfseng, 2000).

Damage to infrastructure such as electric power systems due to natural hazards needs to be reduced as recovery period is longer when the system is severely damaged which in turn can exponentially increase losses (Tierney et al., 1999). As such, cost-effective risk mitigation strategies need to be identified. This requires a comprehensive risk assessment framework that incorporates hazard identification and characterization, consequence analysis, risk evaluation, and risk mitigation. Such framework can be used for pre-disaster preparation, mitigation, and post-disaster response planning. Additionally, such framework is required to guide decision makers to prioritize investment on risk mitigation strategies in the face of limited resources. 
Studies have been conducted towards risk assessment for electric power systems subjected to natural hazards. The effectiveness of various mitigation strategies such as targeted hardening has also been studied (e.g. Han et al. (2013), Shafieezadeh et al. (2014a), Bjarnadottir et al. (2013), Ryan et al. (2014b)). Brown (2009) studied the hardening of 10\% of distribution poles in Texas and estimated the net benefit derived from it. Bjarnadottir et al. (2014) studied targeted hardening of distribution poles in Florida using four strategies: (i) replacement of poles that reach threshold of strength; (ii) replacing failed poles with poles that are one class stronger; (iii) replacing poles that fail with stronger poles plus replacing poles that reach strength threshold; and (iv) proactive measures for foreshore locations by using stronger poles. The above studies, however, focused on componentlevel risk assessment.

Similarly, studies on the potential impact of climate change on risk of power system subjected to hurricanes are limited to component-level performance (e.g. Francis et al. (2011), Bjarnadottir et al. (2013), Bjarnadottir et al. (2014)). Francis et al. (2011) presented a hybrid economic input-output life cycle cost analysis method for evaluating the costeffectiveness of climate change adaptation strategies of distribution systems. Failure of distribution poles, spans, and pad-mounted transformers was considered in the study. The effect of climate change was modeled through the use of count regression analysis and data mining techniques that describe the relationship between climate variability and North Atlantic tropical cyclone counts in the U.S.

Multi-hazard assessment and mitigation were investigated for residential construction ( $\mathrm{Li}$ \& Ellingwood, 2009), bridges (Kameshwar \& Padgett, 2014), and commercial buildings (Wen \& Kang, 2001). While development of multi-hazard risk analysis framework for buildings and bridges have been ongoing in recent years, risk analysis of spatiallydistributed civil infrastructure systems such as electric power and water systems have so far been limited to mostly single-hazard considerations (e.g. Adachi and Ellingwood 
(2010), Winkler et al. (2010), Song and Ok (2010), Duenas-Osorio and Hernandez-Fajardo (2008), Kim and Kang (2013), Ryan et al. (2014a)).

Davis and Clemmer (2014) suggested shifting to renewable energy sources to diversify the electric system and make it more resilient. This is because the use of renewable energy can turn the power system to smaller-scale and more distributed system. Renewable energy sources can be used for smart grid systems where generation and transportation of electricity can be decentralized and failure in one part of the system will not affect other parts. However, though the use of renewable sources of energy can help in improving the resilience of power systems, the current extensive system needs upgrading and strengthening as it is likely to stay in place for years to come before the gradual adaptation of smart grid systems.

Based on the review of existing literature, the following observations can be made:

1. While component-level risk assessment methodologies have been well established, efficient utilization of limited risk mitigation resources requires identifying critical parts or components of a system that when strengthened, will have a greater impact on overall system reliability. To determine the critical parts or components of a system, some form of component importance measure is required. This, in turn, requires system reliability modeling that relates structural components failure and power delivery.

2. Previous research on climate change impact did not couple climate change modeling and system reliability. Decision making regarding appropriate climate change adaptation strategies based on component-level risk analysis might not be accurate.

3. Damage to electric power systems can lead to significant indirect monetary loss to residential, commercial, and industrial customers. However, most existing studies 
did not consider indirect losses in the form of societal economic losses due to power outages.

4. Risk analysis of electric power systems has so far been limited to mostly singlehazard considerations. As these systems usually cover large areas and can be subjected to multiple hazards within their lifetime, there is a need to develop a framework to study the impact of multiple hazards on such systems. This is essential for decision making regarding investment in long-term mitigation of risks due to all possible hazards that can affect the system over its entire lifespan. There is also a need to investigate the cost-effectiveness of mitigation strategies in reducing the overall risks to infrastructure that are vulnerable to multiple hazards. This requires a comprehensive multi-hazard risk-based approach.

\subsection{Research Objectives}

The main objective of this dissertation is to develop a system-level risk-based framework for assessment and strengthening of electric power systems subjected to natural hazards. The focus of the framework is on the distribution as well as the transmission parts of the electric power system. The natural hazards considered are hurricanes and earthquakes, as well as the combined impact of both on the life cycle cost of systems located in areas vulnerable to both hazards. Specific objectives include:

1. Develop a probabilistic system reliability approach that relates structural components failure with power delivery for radially operated distribution systems as well as networked transmission systems.

2. Develop and demonstrate a framework for risk-based assessment and strengthening of distribution systems subjected to hurricanes. 
3. Incorporate climate change model to risk assessment of power systems subjected to hurricanes to study the impact of variation in both intensity and frequency of future hurricanes.

4. Develop and demonstrate a framework for multi-hazard risk assessment of electric power systems subjected to independent non-concurrent hazards through their lifespan.

5. Propose and investigate the cost-effectiveness of various risk mitigation strategies for distribution and transmission systems considering both direct and indirect costs.

The developed framework can be utilized in decision making regarding the design of new electric power systems, investigating the effectiveness of various mitigation in terms of system reliability improvement and monetary benefit, comparing various mitigation strategies, as well as prioritizing investment in instances of competing hazards and limited resources.

\subsection{Organization of Dissertation}

This dissertation has been organized into nine chapters. The content of each chapter is outlined below:

Chapter 1 introduces the subject, discusses the need for risk assessment of electric power systems subjected to natural hazards, reviews existing literature on the subject, and highlights the motivation for the research. Finally, the objectives of the research are presented.

Chapter 2 gives a general background on electric power systems and discusses the different sub-systems that work together to deliver power to customers. Natural 
hazards, specifically hurricanes and earthquakes, are introduced together with a discussion of different regions of the U.S. vulnerable to such hazards. Finally, the concept of infrastructure risk assessment is introduced.

Chapter 3 develops a topological-based system reliability approach for distribution and transmission systems. This includes discussion of system reliability theory, modeling accessibility of system components in radial and networked systems, and line failure models.

Chapter 4 presents a framework for studying the effectiveness of targeted hardening strategies for distribution systems subjected to hurricanes. The framework includes probabilistic and scenario-based hazard modeling, time-dependent component fragility model, component importance evaluation, and cost analysis. The framework is demonstrated using a notional distribution system assumed to be located in Florida.

Chapter 5 extends the framework from chapter 4 to integrate the potential impact of climate change on hurricane risk. Several climate change scenarios are integrated into a hurricane simulation model to investigate the impact of variation in both intensity and frequency of hurricanes on risk. Cost-effectiveness of adaptation strategies is also evaluated using a notional distribution system.

Chapter 6 evaluates the effectiveness of adding redundancy to distribution systems to reduce hurricane risk by constructing additional distribution lines with normally open (NO) switches to connect various independent substations. A system consisting of 20 independent distribution systems in central Florida is used.

Chapter 7 presents a framework for multi-hazard risk assessment of electric power systems subjected to hurricanes and earthquakes. The framework includes probabilistic and scenario-based hazard modeling and different methods of multi-hazard risk 
assessment. A notional electric power system assumed to be located in Charleston, SC, New York, NY, and Seattle, WA, is used to demonstrate the proposed framework.

Chapter 8 extends the framework in Chapter 7 to investigate the effectiveness of various multi-hazard risk mitigation strategies. Probabilistically weighted deterministic hazard scenarios approach is also introduced. Finally, a new component importance measure appropriate for networked systems such as transmission systems is developed. A notional electric power system is used to investigate the cost-effectiveness of the proposed mitigation strategies.

Chapter 9 summarizes findings of the previous chapters, states conclusions from this study, and suggests future avenues for research in the area of risk assessment of infrastructure systems subjected to natural hazards. 


\section{Background}

\subsection{Electric Power Systems}

The electric power system can be broadly divided into three subsystems: generation, transmission, and distribution. The basic structure of a power system showing these subsystems is shown in Figure 2.1. Electric power is produced by generating units which convert mechanical energy into electricity. The generation plants produce electricity by using fossil fuels, nuclear energy, wind, hydro, or solar energy. Power plants produce electricity at a line-to-line voltage of between $11 \mathrm{kV}$ and $30 \mathrm{kV}$ (Brown, 2008). However, this range of voltage is not sufficiently high to transport electricity over long distances. Hence, generation substations are used to step up the voltage to transmission levels.

Electric power from generating units is carried by the transmission system over long distances to the distribution system. The voltage levels for the transmission system ranges from $69 \mathrm{kV}$ to as high as $1100 \mathrm{kV}$ in the transmission and sub-transmission lines in the US (Brown, 2008). The sub-transmission system carries electric power at lower voltages and over shorter distances than the transmission system and is used to connect the transmission system to multiple distribution systems (Kassakian et al., 2011). The transmission system is composed of power lines and substations. The conductors in the power lines are supported by structures that can be lattice steel towers or $\mathrm{H}$-frames for transmission systems and single pole structures for sub-transmission systems.

Topologically, the transmission and sub-transmission systems have mesh-like designs to provide multiple paths from one node (substation) to another. This increases flexibility and improves reliability so that power can be delivered to loads even when a transmission line, substation, or generating unit goes offline. Transmission security assessment is routinely carried out to determine whether a system can deliver peak demand after one or more pieces

of equipment or components are disconnected (Brown, 2008). After removing a piece of 
equipment, power flow is run to check if all voltage levels are within limits and equipment are not loaded above emergency ratings. This ensures that the transmission system is at least $N-1$ secure at all times. 


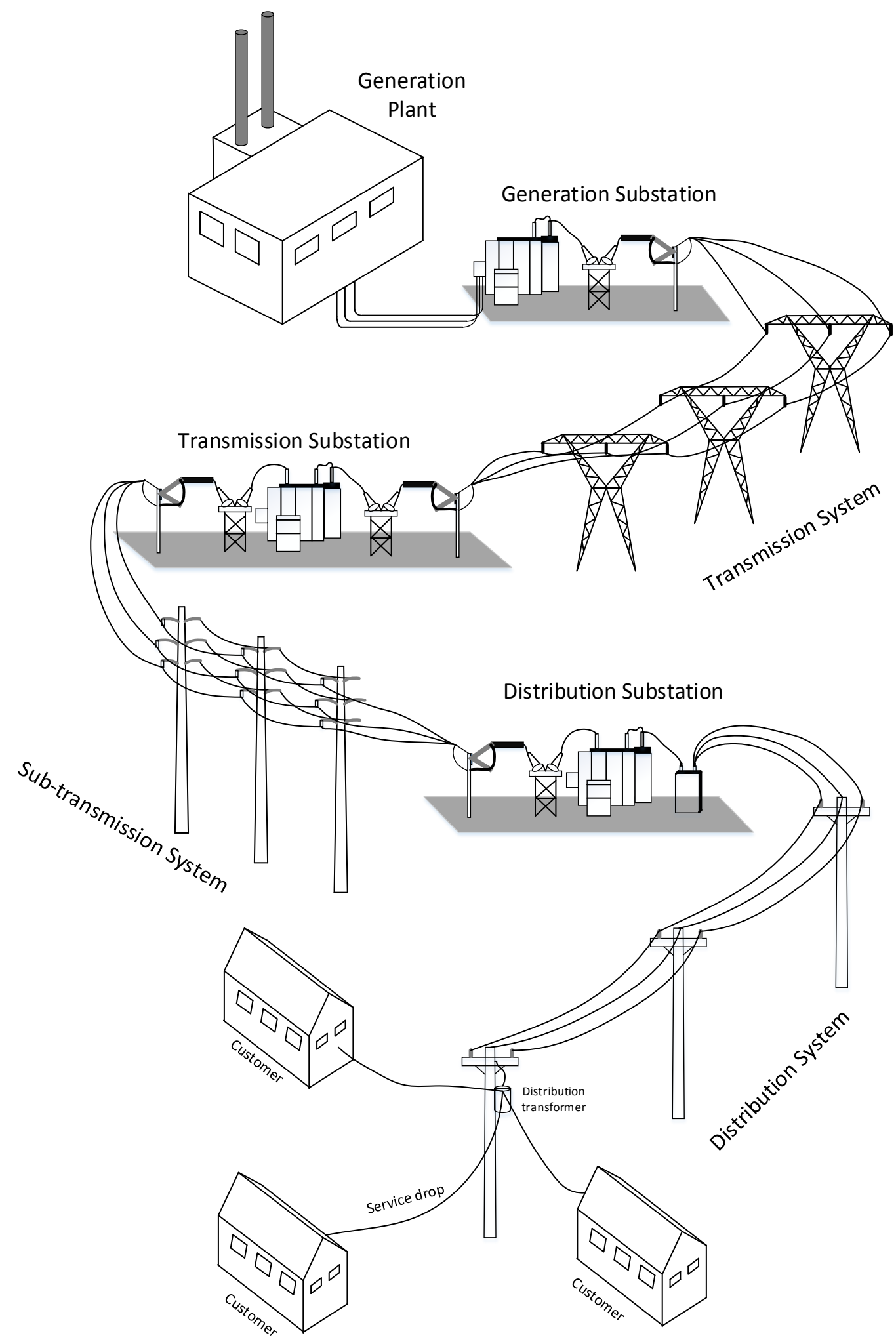

Figure 2.1 Schematic of an electric power system 
The distribution system starts from a distribution substation that is fed by one or more subtransmission lines (Kersting, 2012). The main function of the distribution substation is to step down the voltage to distribution level by utilizing a transformer. The stepped down power leaves the substation through feeders that can be either overhead or underground system. The feeders eventually branched off to smaller lateral lines that deliver power directly to customers. Overhead distribution systems utilize wires that are carried by wood, steel or concrete poles that are 30 to $40 \mathrm{ft}$ high and spaced 100 to $150 \mathrm{ft}$ in the suburbs and 300 to $400 \mathrm{ft}$ in rural areas (Short, 2006). The voltage is usually between $4.16 \mathrm{kV}$ to 34.5 $\mathrm{kV}$ in the distribution system and is mostly carried by Aluminum Conductor Steel Reinforced (ACSR) and All Aluminum Conductor (AAC) wires (Brown, 2008).

Unlike the transmission and sub-transmission systems, the topology of the distribution system is mostly radial or radially operated in the U.S. (Kersting, 2012; Brown, 2008). Radial implies there is a unique path for power flow from a substation to each customer. Radially operated distribution systems have ring (or loop) topology where there is more than one path from the power source to customers. The radial nature of the system is maintained by using normally open (NO) switches which can be closed to provide an alternative path in the case of a fault. This increases reliability by allowing customers downstream of a fault to receive power using a combination of NO switches and sectionalizing switches.

Electric power grids are among the most extensive and complex engineering systems in modern societies. The generation, transmission, and distribution systems work together to supply electricity to millions of different types of customers ranging from residential, commercial, and industrial customers. Table 2.1 gives a summary of some of the characteristics of the U.S. electric grid. 
Table 2.1 General information on the U.S. electric grid

\begin{tabular}{lll}
\hline \hline Description & Approximate value & Source \\
\hline Total number of customers & 147 million & EIA (2016b) \\
Number of residential customers & 128 million (37\% of consumption) & EIA (2016b) \\
Number of commercial customers & 17.8 million (36\% of consumption) & EIA (2016b) \\
Number of industrial customers & $839,000(27 \%$ of consumption) & EIA (2016b) \\
Number of transportation customers & $79(0.2 \%$ of consumption) & EIA (2016b) \\
Total revenue (2014) & $\$ 393$ million & EIA (2016b) \\
Revenue from residential customers & $45 \%$ of total & EIA (2016b) \\
Revenue from commercial customers & $37 \%$ of total & EIA (2016b) \\
Revenue from industrial customers & $18 \%$ of total & EIA (2016b) \\
Revenue from transport customers & $0.2 \%$ of total & EIA (2016b) \\
High voltage transmission lines & 200,000 miles & NERC (2015) \\
Low voltage distribution lines & 6 million miles & Kassakian et \\
Primary energy consumed & $40 \%$ of total & al. (2011) \\
\hline \hline
\end{tabular}

\subsection{Natural Hazards}

Natural hazards that threaten the power system include hurricanes, earthquakes, floods, ice storms, severe thunderstorms, tornadoes etc. Each of these hazards causes failure in a different way and to different parts of the power system. The duration of the resulting interruption also usually depends on the type of hazard with some causing considerably long interruptions. Utility companies are required to submit reports to both U.S. Department of Energy (DOE) and the North American Electric Reliability Council (NERC) when there is a sufficiently large disturbance in their territories. Between 1984 and 2006, there were 438 events that resulted in interruptions of more than $300 \mathrm{MW}$ or affected at least 50,000 customers. A summary of the causes of such interruptions is given in Table 2.2 (Hines et al., 2009). 
Table 2.2 Summary of causes of major power outages in the U.S.

$(1984-2006)$

\begin{tabular}{lccc}
\hline Cause of outage & \% of events & $\begin{array}{c}\text { Mean MW } \\
\text { lost }\end{array}$ & $\begin{array}{c}\text { Mean number of } \\
\text { customers affected }\end{array}$ \\
\hline Wind/rain & 31.4 & 679 & 235,840 \\
Equipment failure & 19.9 & 767 & 248,643 \\
Ice storm & 11.1 & 1664 & 431,184 \\
Hurricane/tropical storm & 10.1 & 2684 & 912,870 \\
Other cold weather events & 8.8 & 1045 & 271,924 \\
Lighting & 8.8 & 794 & 200,617 \\
Operator error & 8.5 & 1226 & 358,440 \\
Fire & 5.6 & 972 & 294,994 \\
Other external cause & 3.6 & 1518 & 823,691 \\
Tornado & 3.6 & 721 & 227,073 \\
Supply shortage & 2.3 & 600 & 896,432 \\
Earthquake & 1.6 & 1124 & 526,260 \\
Intentional attack & 0.7 & 2154 & 165,000 \\
\hline \hline
\end{tabular}

It can be seen from Table 2.2 that even though hurricanes and tropical storms constitutes only about $10 \%$ of the total number of events, they resulted in the highest damage in terms of mean megawatts (MW) lost and mean number of customers affected. While earthquakes constitute only about $1.6 \%$ of events, which is second to last, they are $4^{\text {th }}$ in terms of average number of customers affected and $6^{\text {th }}$ in terms of mean MW lost. Considering only natural hazards, earthquakes are only second to hurricanes/tropical storms in terms of number of customers affected and third in terms of MW lost. Generally, natural hazards result in relatively widespread damage to electric power systems and longer repair times. 


\subsubsection{Hurricanes}

All circulating weather systems over tropical waters are generally referred to as tropical cyclones. There are three classes of tropical cyclones: tropical depressions, tropical storms, and hurricanes. Tropical depressions have maximum sustained winds $\leq 38 \mathrm{mph}$ while tropical storms have maximum sustained winds of 39 to $73 \mathrm{mph}$. Hurricanes are intense systems with a well-defined circulation and maximum sustained winds $\geq 74 \mathrm{mph}$ (NOAA, 2016a). In western pacific, hurricanes are referred to as "typhoons". All Atlantic and Gulf coastal areas of the US are prone to hurricanes. On average, the US coastline is hit by five hurricanes in a typical 3-year period, of which two will be major ( $\geq$ category 3 ) (NOAA, 2016a).

The Saffir-Simpson Hurricane Scale is used to categorize hurricanes into five categories based on their 1-minute sustained wind speeds. Table 2.3 shows the five categories of hurricanes and their range of wind speeds as well as the total number of hurricanes that directly hit the U.S. between 1851 and 2015 (NOAA, 2016c, 2016b; Jarrell et al., 2001).

Table 2.3: Saffir-Simpson hurricane scale and summary of hurricanes that hit the U.S.

\begin{tabular}{|c|c|c|c|}
\hline \multirow{2}{*}{ Category } & \multicolumn{2}{|c|}{ Sustained wind speed } & \multirow{2}{*}{$\begin{array}{c}\text { Number of hurricanes } \\
(1851-2015)\end{array}$} \\
\hline & mph & $\mathbf{m} / \mathbf{s}$ & \\
\hline 1 & $74-95$ & $33-42$ & 117 \\
\hline 2 & $96-110$ & $43-49$ & 76 \\
\hline 3 & $111-129$ & $50-58$ & 76 \\
\hline 4 & $130-156$ & $58-70$ & 18 \\
\hline 5 & $\geq 157$ & $>70$ & 3 \\
\hline
\end{tabular}


Hurricanes are among the major causes of power outages in the U.S. Since 2002, 58\% of power outages and $87 \%$ of outages affecting 50,000 or more customers were caused by severe weather such as hurricanes, thunderstorms, and blizzards (DOE, 2016). Table 2.4 summarizes hurricanes that damaged the U.S. electric power system and the number of customers affected (DOE, 2016). Hurricanes rarely affect power generation stations and cause little to moderate damage to the transmission system. This is because generation and transmission systems are designed to withstand high wind loads. The distribution system, however, can be significantly affected by hurricanes. Much of the damage to the distribution system is done by high winds which can uproot distribution poles and damage distribution lines due to flying debris or falling trees. For example during the 1989 Hurricane Hugo, falling trees knocked out thousands of distribution poles and lines cutting power to over 1 million customers (NOAA, 1990).

Table 2.4 Summary of damage to power systems caused by hurricanes

\begin{tabular}{llll}
\hline Year & Hurricane & $\begin{array}{l}\text { Approximate number } \\
\text { of customers affected }\end{array}$ & $\begin{array}{l}\text { Approximate MW } \\
\text { lost }\end{array}$ \\
\hline 2002 & Hurricane Lily & 242,000 & Not Reported (NR) \\
2003 & Hurricane Isabel & 3.9 million & 15,000 \\
2004 & Hurricane Charley & 2 million & 4,200 \\
2004 & Hurricane Frances & 4.2 million & 14,700 \\
2004 & Hurricane Ivan & 1.3 million & 2,000 \\
2004 & Hurricane Jeanne & 1.2 million & 4,300 \\
2005 & Hurricane Dennis & 278,000 & 96 \\
2005 & Hurricane Katrina & 2 million & NR \\
2005 & Hurricane Ophelia & 60,000 & 215 \\
2005 & Hurricane Rita & 2 million & 3,200 \\
2005 & Hurricane Wilma & 3.7 million & 10,800 \\
2008 & Hurricane Gustav & 1.3 million & NR \\
2008 & Hurricane Ike & 5.9 million & NR \\
2012 & Hurricane Isaac & 1.4 million & NR \\
2012 & Hurricane Sandy & 5.9 million & NR \\
\hline \hline
\end{tabular}




\subsubsection{Earthquakes}

Earthquakes are among the most devastating natural hazards. The extent of damage from earthquakes depends on population density and level of development of infrastructure such as power systems in an area. Most of the US has some seismic risk with some areas being more prone than others. Figure 2.2 shows the U.S. seismic hazard map with PGAs having a return period of 2475 years (USGS, 2016). As discussed in the previous section, the entire east coast of the U.S. is vulnerable to hurricane hazard. It can be seen from Figure 2.2 that some areas on the east coast such as South Carolina are also vulnerable to seismic hazard. Multi-hazard risk analysis approach is therefore required for such areas.

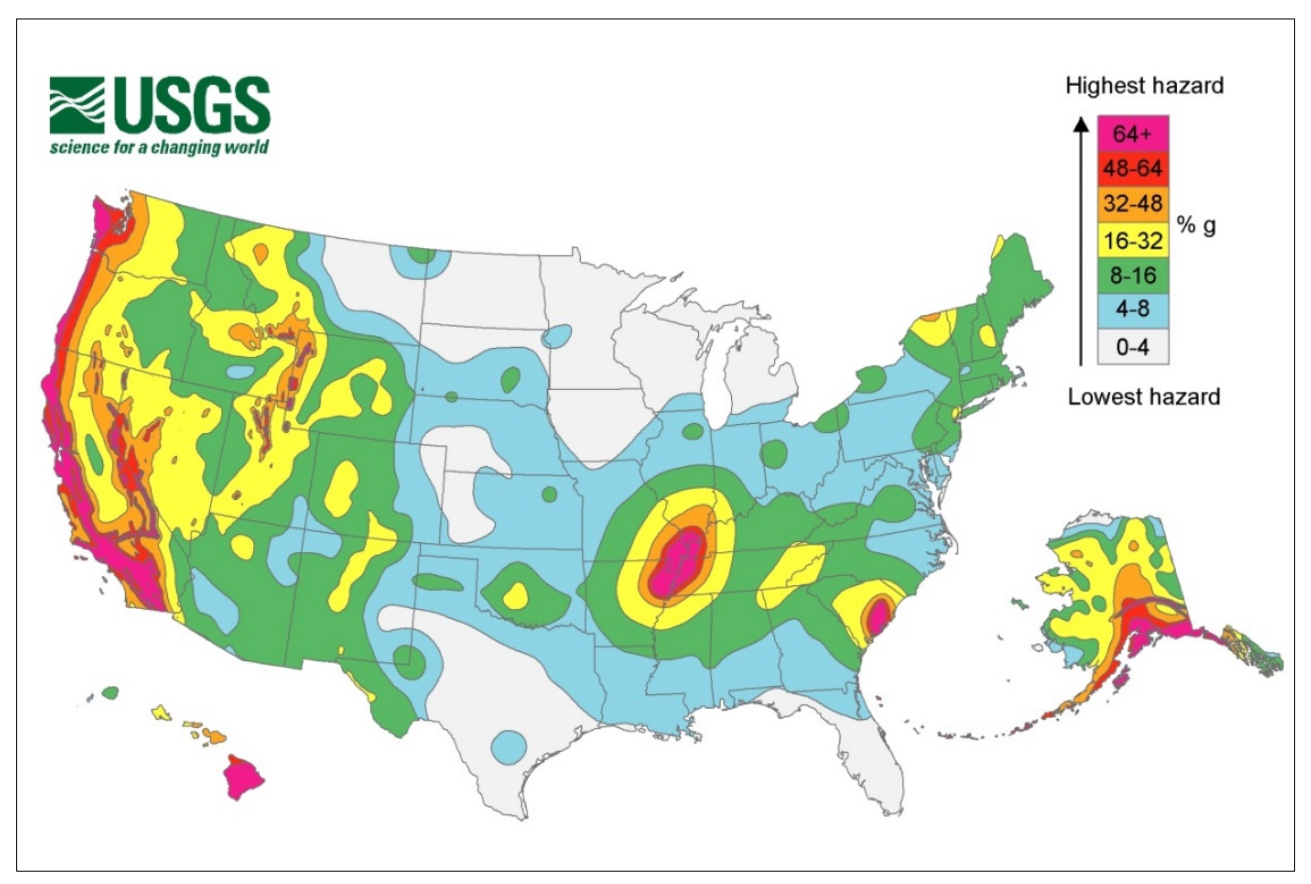

Figure 2.2 U.S. seismic hazard map (PGA, $2 \%$ in 50 years)

[Image courtesy of USGS (2016). See Appendix A]

Earthquakes can cause damage to power generation facilities depending on the intensity of the earthquake and size of the power plant. Most power plants are usually designed to have 
good seismic resistance. The 1989 San Francisco earthquake caused damage to the Moss Landing Facility which is a large generating plant located about 20 miles from the epicenter (U.S. Congress, 1990). Transmission towers are rarely damaged by the actual shaking of the ground during earthquakes. This is because the towers are designed for severe loads such as combined wind and ice, extra loads due to the collapse of adjacent towers and so on. Instead, the damage is mostly due to foundation failures caused by landslides, ground fracture and liquefaction (Shinozuka et al., 2005).

Substations have several brittle components that have considerable mass which makes them prone to earthquake damage (Vanzi, 1996; Eidinger \& Kempner, 2012). Unanchored rail-supported transformers in substations can fall from elevated platforms which can result in severe damage. Lack of adequate slack in conductors connecting equipment is another source of damage in substations. Current design guidelines require anchoring of substation equipment to the foundation or first-support, either by welding or bolting. There are, however, a large number of existing substation equipment across the US that are not retrofitted and anchored (Knight \& Kempner Jr, 2009b). The 1971 San Fernando earthquake, for example, damaged many high-voltage substations causing power outages (U.S. Congress, 1990). The 2011 Christchurch earthquake in New Zealand also caused severe damage to substations due to ground shaking and liquefaction causing a power outage for several days (Massie \& Watson, 2011). Earthquakes usually cause little damage to distribution lines because of their sizes and nature. A summary of some earthquakes and resulting damage to electric power systems is shown in Table 2.5. 
Table 2.5 Summary of damage to power systems caused by earthquakes

\begin{tabular}{llll}
\hline \hline Year & Earthquake & $\begin{array}{l}\text { Approximate number of } \\
\text { customers affected }\end{array}$ & Source \\
\hline 1989 & Loma Prieta earthquake & 1.4 million & NRC (1994) \\
1994 & Northridge earthquake & 2.5 million & Dong et al. (2004) \\
2001 & Nisqually earthquake & 217,000 & Creager et al. (2001) \\
2003 & San Simeon earthquake & 109,000 & DOE (2016) \\
2010 & Eureka earthquake & 28,000 & Valencia et al. (2010) \\
\hline \hline
\end{tabular}

\subsection{Infrastructure Risk Assessment and Management}

In general terms, risk is defined as a measure of probability and severity of harm or adverse effects (Lowrance, 1976). In the context of infrastructure risk assessment, risk can be defined as the potential for loss or damage to infrastructure due to exposure to uncertain hazards. Uncertainties are inherent in both the occurrence of future hazard events as well as the consequent losses. Prediction of occurrence of hazard events is usually based on available historical data. Therefore risk analysis is prospective, anticipating scientifically credible future scenarios (Cardona et al., 2012). Due to uncertainties in both hazard occurrence and consequent losses, risk analysis of infrastructure is usually based on probabilistic formulations that incorporate the uncertainties into the risk analysis. In simple mathematical form, risk can be expressed as (Ayyub et al., 2009):

$$
\text { Risk }=\text { probability of event } \times \text { vulnerability } \times \text { consequence }
$$

The probability of event is a measure of the likelihood of occurrence of hazard event of a given intensity and in a given area and time period. It is, therefore, a function of hazard source, location, and intensity. Vulnerability is the susceptibility of exposed components or systems to damage. For structures and infrastructure system components, this can be quantified through fragility analysis. Consequence is the potential loss due to damage 
caused by the hazard. It can be measured in monetary terms, casualties, downtime, or power outage and duration in the case of electric power systems.

Assessment of risk involves asking three basic questions (Kaplan, 1997):

1. What can go wrong?

2. How likely is it to go wrong (probability/frequency)

3. What are the consequences?

To answer the above three questions in the context of civil infrastructure risk assessment, the framework in Figure 2.3 is developed. The first stage of any risk assessment involves identification of any or all hazards that can impact a system and quantifying the hazard in terms of intensity and frequency. The second stage is exposure analysis which involves identifying assets that are exposed to the identified hazards. The likelihood of damage and the consequence of such damage is then quantified. The final stage of the framework involves evaluating whether the level of risk is within acceptable/tolerable limits. This last stage of the risk assessment framework leads to the decisions regarding mitigation and management of risk.

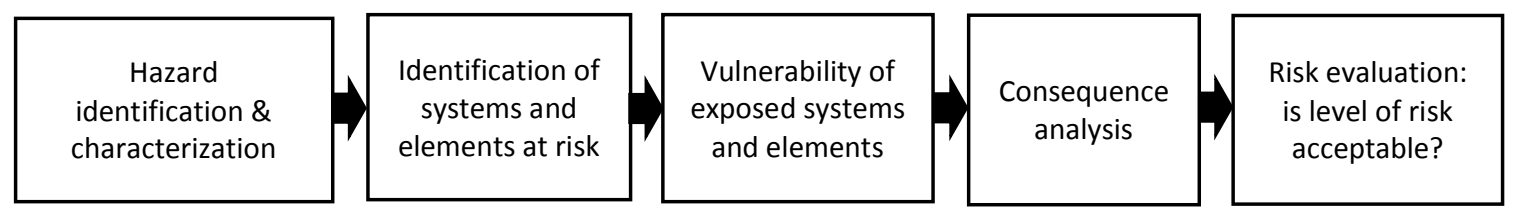

Figure 2.3 Risk assessment methodology

Risk mitigation involves reducing one or more of the three components of risk in Equation (1). For infrastructure subjected to natural hazards, reducing the probability of occurrence of the hazard events is not feasible. Hence, mitigation strategies usually involve reducing vulnerability and/or consequence. Vulnerability can be reduced by improving the 
reliability of infrastructure systems through hardening of existing components, introducing redundancy in the system etc. One way to reduce consequence is to provide alternative to the exposed infrastructure. For example, microgrids and distributed generation can reduce power outage during or after natural disasters in case of damage to the main power grid.

Risk management can be summarized in three questions (Haimes, 2015; Ezell et al., 2000):

1. What can be done?

2. What options are available and what are their corresponding cost, risks, and benefits?

3. What will be the impact of current risk management decisions on future options?

Risk management involves four main steps as shown in Figure 2.4: (1) estimating the level of risk under the status quo (without mitigation); (2) identifying possible risk mitigation strategies and their associated cost; (3) re-evaluating risk to estimate reduction in losses due to mitigation strategies; and (4) calculating the cost-effectiveness of mitigation measures. The risk management framework shown in Figure 2.4 forms the basis for all the frameworks developed in this dissertation for different sub-systems of the electric power system subjected to different natural hazards.

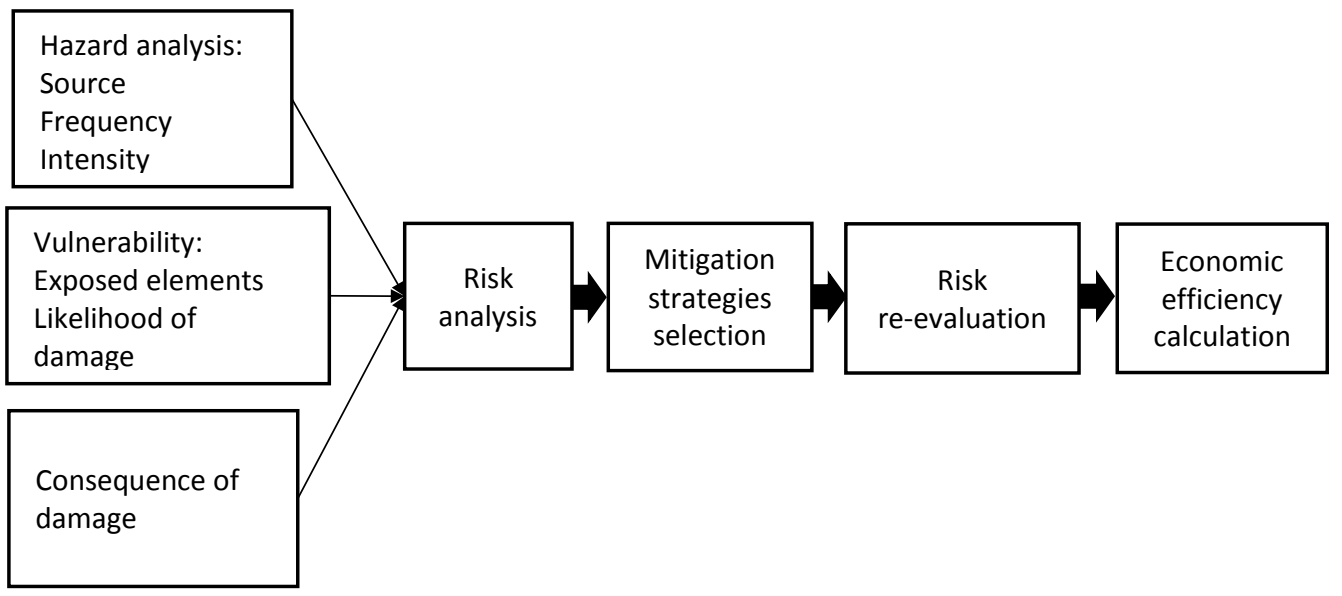

Figure 2.4 Risk management framework 


\section{System Reliability Model}

Civil infrastructure systems are made up of components that work together to serve a specific purpose. Electric power systems, for example, rely on components in the generation, transmission, and distribution sub-systems to deliver electricity to customers. The functionality of the system at any particular time depends on the state of the components. Therefore, risk analysis of such a system requires consideration of the interaction among its components.

A critical part of any system-level risk analysis process is quantifying the consequence of damage to the system. This can be done using various performance measures such as system reliability. System reliability is defined as the ability of a system to perform a required function for a stated period of time under given environmental and operating conditions (Rausand \& Høyland, 2004). System reliability theory can be used to assess the risk to infrastructure systems subjected to natural hazards, evaluate the efficiency of disaster mitigation methods, design maintenance plans, and design layout of system components.

For electric power systems, models of performance measure can range from purely topological-based models to complex alternating current (AC) power flow models. Topological- or connectivity-based models only consider the manner in which system components are arranged (topology) to describe the behavior of the system. Physical constraints that govern power flow within the system is ignored. Power flow-based models, on the other hand, take into account the physics of power flow, power capacity limits of components and other engineering details of the system (LaRocca et al., 2014).

Topological-based models have two main advantages: (i) they are computationally efficient especially for complex systems or in a case where system performance under various scenarios is desired, and (ii) significantly less data about a system is required to evaluate reliability. While power flow-based models provide more accurate description of 
system performance, they are computationally complex and often impractical (LaRocca et al., 2014; Duenas-Osorio \& Hernandez-Fajardo, 2008; Kim \& Kang, 2013; Cavalieri et al., 2014; Albert et al., 2004). Furthermore, detail information about engineering properties of system components is required for such analysis. As this study focuses mainly on structural components of the power system, which define the topology, the topological-based method will be used.

\subsection{Review of Existing Topological-Based Methods}

While topological-based system performance models have not been applied to distribution systems, few models for transmission (networked) systems have been proposed. Albert et al. (2004) proposed a measure of system performance termed connectivity loss $\left(C_{L}\right)$ for a grid-like system. The method was developed to investigate the impact of removing nodes (generators and substations) from the North American power grid and to determine whether the grid is reliant on a small set of hubs whose removal will cause large-scale breakdown of the power grid. Nodes are modeled with binary functions, i.e., either functioning or failed. The connectivity loss is given by Equation (3.1). The connectivity loss measures the decrease in the ability of a distribution substation to receive power from the generators.

$$
C_{L}=1-\frac{1}{N_{D}} \sum_{i}^{N_{D}} \frac{N_{G}^{i}}{N_{G}}
$$

where $N_{G}$ is the total number of generators; $N_{D}$ is the total number of distribution substations; and $N_{G}^{i}$ is the number of generators connected to substation $i$.

LaRocca et al. (2014) introduced a topological-based system performance measure termed efficiency, which is calculated based on the shortest path between a pair of nodes in a network. The method was demonstrated by randomly removing nodes and edges from the 
network and studying the impact on efficiency. The efficiency of a network is given by Equation (3.2).

$$
E=\frac{1}{N(N-1)} \sum_{i \in N_{F}, j \in N_{L}} \frac{1}{d_{i j}}
$$

where $N$ is the total number of nodes in the network; $N_{F}$ is the set of in-feed nodes; $N_{L}$ is the set of load nodes; and $d_{i j}$ is the length of the shortest path between node $i$ and node $j$. In another approach, Johansson et al. (2007) used power connection loss (PCL) to evaluate system performance. PCL, given by Equation (3.3), is defined as the aggregate load at nodes that do not have any connection to a power source.

$$
P C L=\sum_{i \in N C} \operatorname{load}_{i}
$$

where $N C$ is the set of nodes that do not have any connection to a power source and load $i$ is the load at node $i$.

The vulnerability of structural components subjected to natural hazards is usually evaluated using fragility curves. Fragility curves give the probability of failure between 0 and 1 for a component given a specific hazard level. The probability of failure is, therefore, a continuous random variable. However, the above methods assigned a probability of failure or probability that power will not be supplied to customers of either 0 or 1 . As such, the above topological methods fail to capture the true stochastic nature of risk assessment under natural hazards. In the following two sections, a topological-based method that overcomes this limitation is presented. 


\subsection{Accessibility of System Components}

The ability of a component in a system to perform its function depends invariably on its reliability as well as the reliability of other components. Even if a system component is not physically damaged after a hazard event, damage to other components can prevent it from performing its intended function. Accessibility of a component is therefore defined here as the probability that commodity (power) will be supplied to the component.

As mentioned in Chapter 2, the topology of distribution and transmission systems differ significantly. Most distribution systems have radial topology such as the one shown in Figure 3.1(a). Power delivery starts from the substation through main feeder lines. The feeders eventually branched off to smaller lateral lines that deliver power directly to customers. Transmission systems, on the other hand, have a networked topology with several paths from one node to another. The accessibilities of the two different topologies are formulated below.

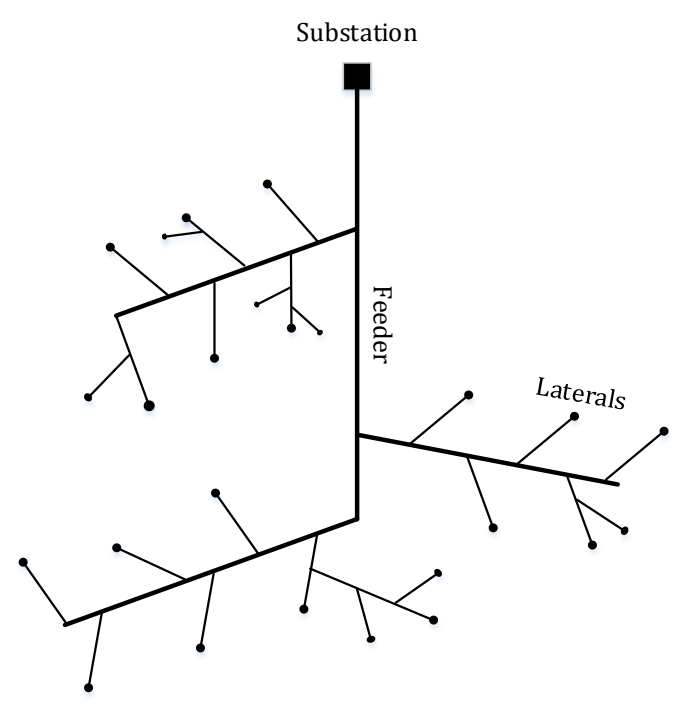

(a)

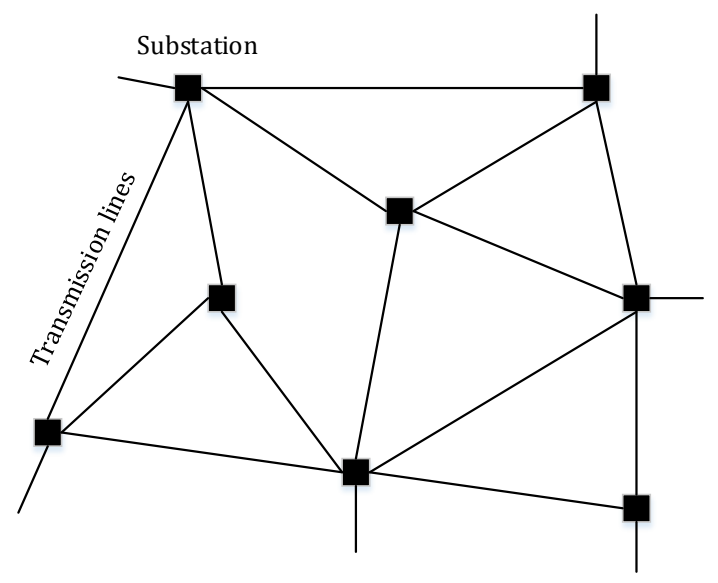

(b)

Figure 3.1 Topology of electric power sub-systems

(a) Distribution system (radial or tree-like) (b) Transmission system (networked or gridlike) 


\subsubsection{Radial Systems}

In radial distribution systems, a line is defined as a switchable section with one or more isolator elements at its ends. Isolator elements, commonly known as sectionalizers, are usually installed at several points within a system so as to allow parts of the system to be isolated in case of any disturbance at any point along a line (Brown, 2008). The presence of isolator elements within a distribution system allows each line to be considered individually as a 'switchable section'. All components in a switchable section have the same reliability characteristics and failure of any component have the same impact regardless of the location of the failed component. Consequently, switchable sections can be reduced to single component equivalent. A failed line is assumed to be isolated from the rest of the system by activating the isolator element upstream of the line.

Due to the radial nature of most distribution systems, the accessibility of lines in the system can be modeled as a series system in which the failure of any line or component along a path can lead to failure of power delivery to lines downstream of the failed line. Consider the simple radial system shown in Figure 3.2. The failure probability of power delivery to each lateral line is calculated by considering power flow. For example, considering line 7, power will be cut off to the line if line 1, 3, 5, 6 or 7 itself fails. The accessibility of line 7 can, therefore, be represented with the reliability block diagram in Figure 3.3. 


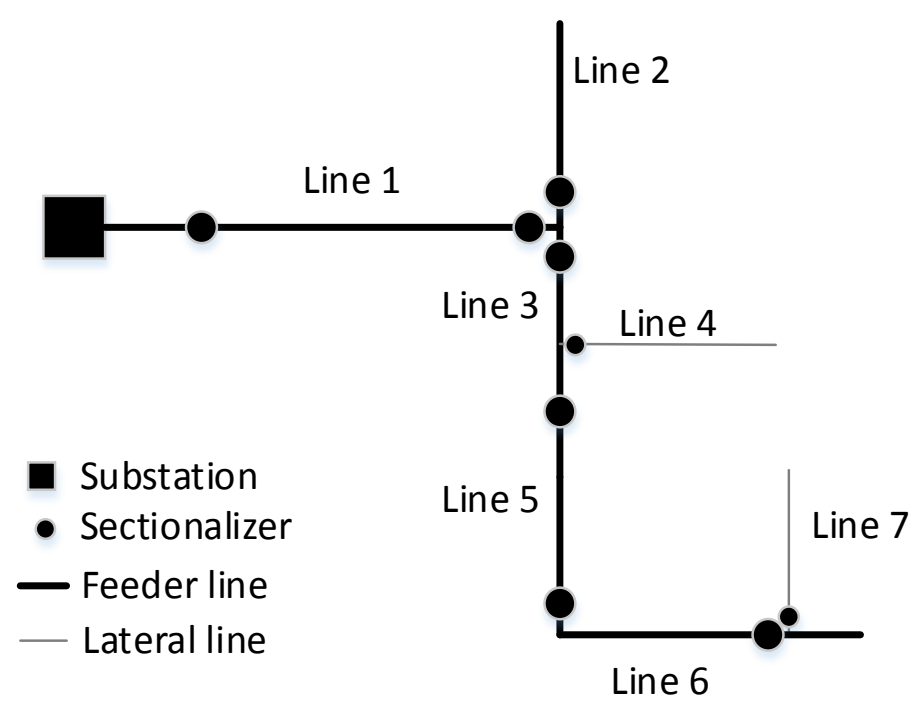

Figure 3.2 Schematic of a radial distribution system

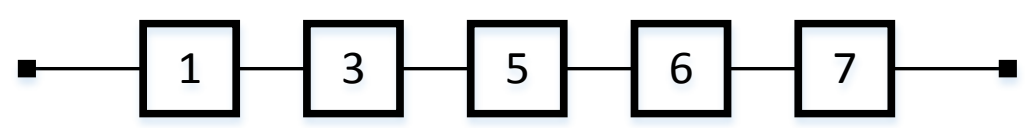

Figure 3.3 Reliability block diagram for power delivery to line 7

The probability that power is not delivered to line 7 (complement of accessibility) is therefore given by Equation (3.4).

$$
Q_{L_{7}}=1-\left[\left(1-P_{f_{L 1}}\right)\left(1-P_{f_{L 3}}\right)\left(1-P_{f_{L 5}}\right)\left(1-P_{f_{L 6}}\right)\left(1-P_{f_{L 7}}\right)\right]
$$

where $P_{f_{L 1}}, P_{f_{L 3}}, P_{f_{L 5}}, P_{f_{L 6}}$, and $P_{f_{L 7}}$ are probabilities of failure of lines $1,3,5,6$, and 7 respectively. Note that in Equation (3.4), the failures of the lines are independent due to the isolator elements.

In a situation whereby a line can be supplied by more than one substation as will be seen in Chapter 6, all paths from source to the line are considered in formulating the accessibility. In systems with more than one power source, normally open (NO) switches 
are used to maintain the radial nature of the system. If one path of power delivery fails, the NO switch can be closed to initiate the flow of electricity through alternative paths. For example, considering the simple schematic in Figure 3.4, power will be cut off to lateral line 4 if line 3 fails, or both lines 1 and 2 fail, or if line 4 itself fails. Failure of power delivery to line 4 can be represented with the fault tree diagram in Figure 3.5.

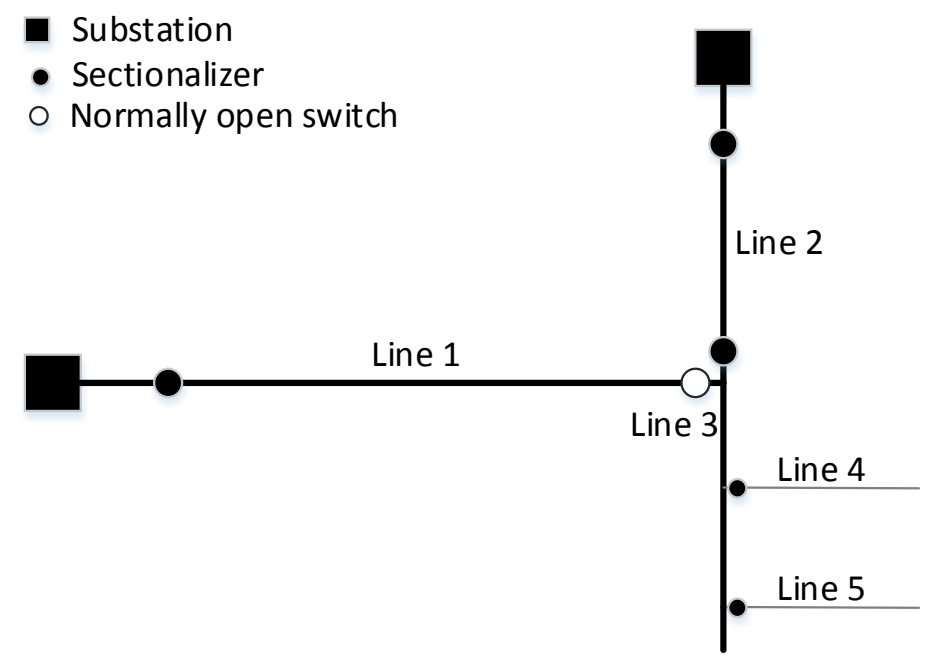

Figure 3.4 Simple schematic of distribution system with redundancy

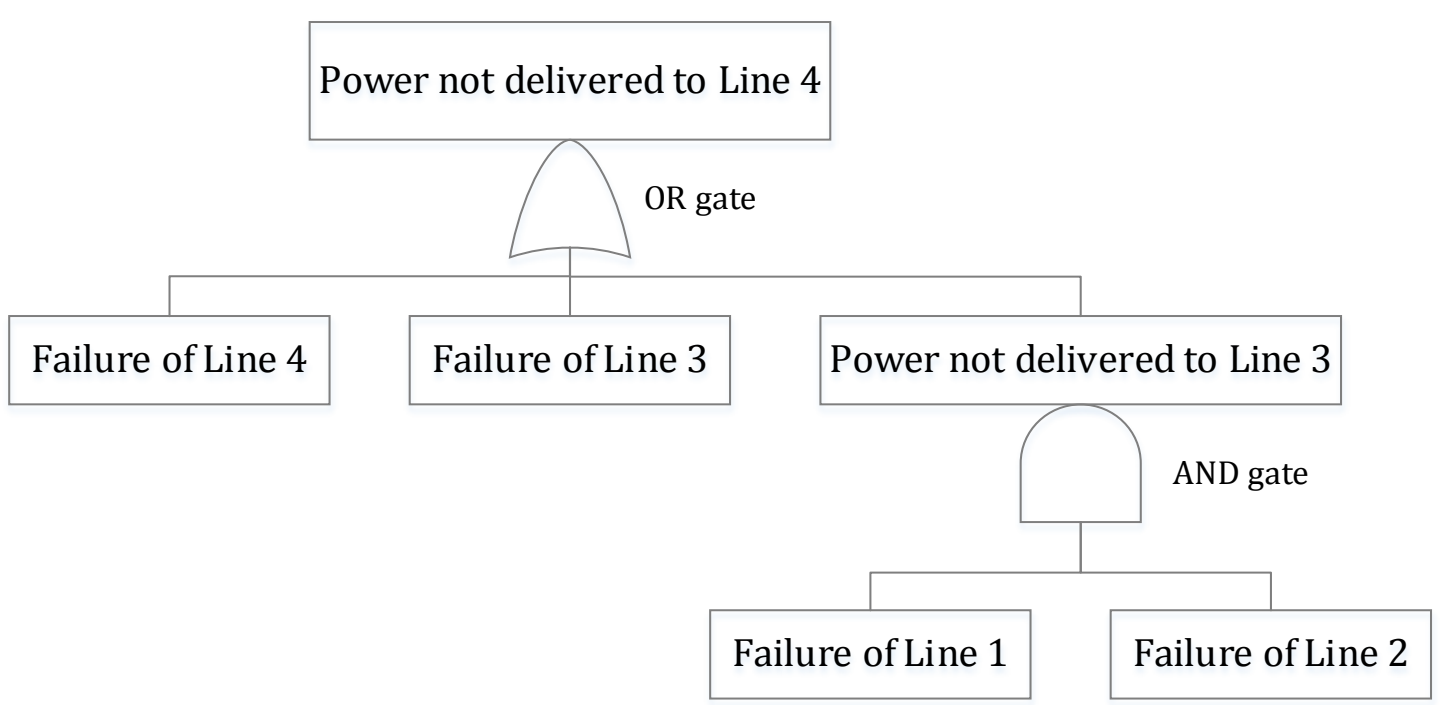

Figure 3.5 Fault tree diagram for failure of power delivery to Line 4 
The probability that power is not delivered to Line 4 is then calculate using Equation (3.5).

$$
Q_{L_{4}}=1-\left[\left(1-P_{f_{L 4}}\right)\left(1-P_{f_{L 3}}\right)\left(1-P_{f_{L 1}} \cdot P_{f_{L 2}}\right)\right]
$$

where $P_{f_{L 1}}, P_{L 2}, P_{f_{L 3}}$, and $P_{f_{L 4}}$ are probabilities of failure of lines 1, 2, 3, and 4 respectively.

\subsubsection{Networked Systems}

Consider the simple schematic in Figure 3.6 with 2 supply stations (gate stations) and 3 demand substations (low voltage substations). Whether substation S1 remains functional depends not only on its own reliability but on the reliability of gate stations G1 and G2, substation S2, as well as transmission lines T1, T3, T4, and T5.

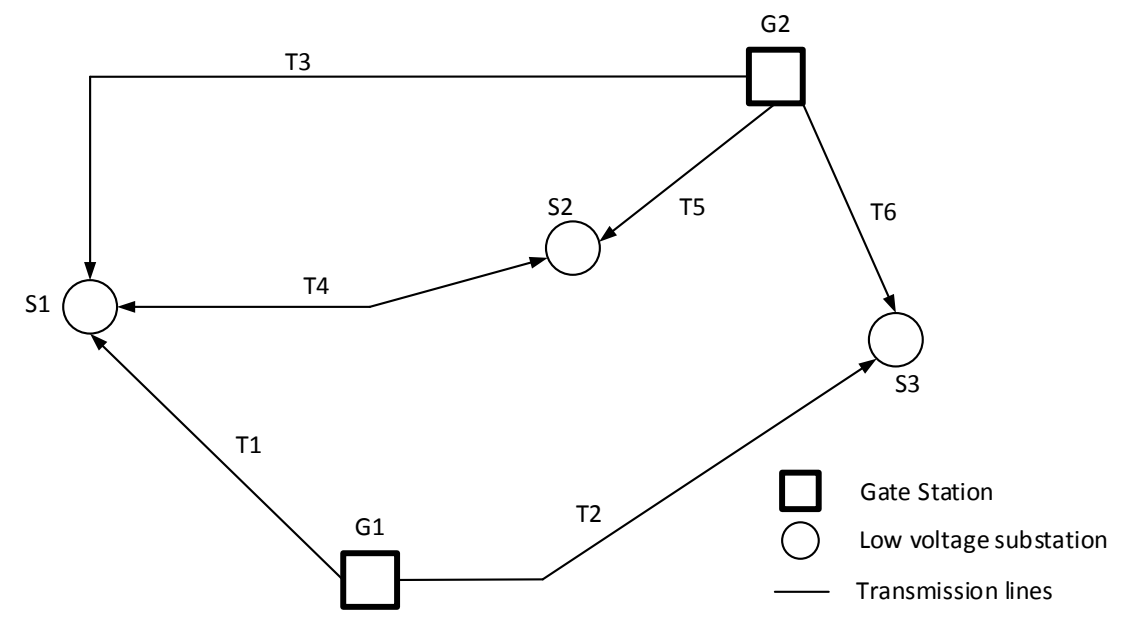

Figure 3.6 Schematic of a networked power system

Now consider a situation where only the edges (transmission lines) are assumed to be vulnerable to failure. The problem of calculating the probability that power is not delivered to any node (substation) reduces to a terminal-pair reliability problem. A terminal-pair 
reliability problem aims to determine the probability of successful communication between any pair of nodes in a network, given the reliability of each edge in the network. In such a case, the accessibility or terminal-pair reliability of any node (which is the complement of the probability that power is not delivered to that node) is the union of the reliability of all the minimal cut sets from supply nodes to the node in question.

A cut set here is defined as the set of components such that if these components fail, the system fails (i.e., power is not delivered to the intended node). A cut set is minimal if, when any component is removed from the set, the remaining components collectively are no longer a cut set.

Considering power delivery to S1 and only edge failure, there are three paths for power flow to S1: (i) through T1, (ii) through T3, and (iii) through T5 and T4. Failure of a combination of one element from each path will result in power not being delivered to S1. The minimal cut sets are, therefore:

1. $\mathrm{T} 1, \mathrm{~T} 3$, and $\mathrm{T} 4$

2. $\mathrm{T} 1, \mathrm{~T} 3$, and $\mathrm{T} 5$

In other words, the system will fail if T1, T3, and T4 fail OR if T1, T3, and T5 fail.

Defining the probabilities of failure of T1, T3, T4, and T5 as $P_{f T 1}, P_{f T 3}, P_{f T 4}$, and $P_{f T 5}$, respectively, and assuming failure of the lines to be independent, the probability that power will not be delivered to $\mathrm{S} 1$ is then:

$$
Q_{S 1}=\left(P_{f T 1} P_{f T 3} P_{f T 4}\right) \cup\left(P_{f T 1} P_{f T 3} P_{f T 5}\right)
$$

Denoting $\left(P_{f T 1} P_{f T 3} P_{f T 4}\right)$ as $P(A)$ and $\left(P_{f T 1} P_{f T 3} P_{f T 5}\right)$ as $P(B)$, 


$$
Q_{S 1}=P(A \cup B)=1-P(\overline{A \cup B})
$$

From de Morgan's rule,

$$
Q_{S 1}=1-P(\bar{A} \cap \bar{B})=1-[1-P(A)] \cdot[1-P(B)]
$$

$A$ and $B$ (minimal cut sets) in the above equation are assumed to be independent. This will be clarified later. Substituting $A$ and $B$ with the original probabilities,

$$
Q_{S 1}=1-\left[1-\left(P_{f T 1} P_{f T 3} P_{f T 4}\right)\right] \cdot\left[1-\left(P_{f T 1} P_{f T 3} P_{f T 5}\right)\right]
$$

The terminal-pair reliability of $S 1$, which is the complement of $Q_{S 1}$ is then

$$
R_{T}=1-Q_{S 1}=\left[\left(1-P_{f T 1} P_{f T 3} P_{f T 4}\right)\left(1-P_{f T 1} P_{f T 3} P_{f T 5}\right)\right]
$$

Considering a situation where only the nodes (substations) are assumed to be vulnerable to failure, power will not be delivered to S1 when S1 itself fails or when both G1 and G2 fail. The probability that power is not delivered to S1 is then given by Equation (3.11) based on the minimal cut sets from source nodes to S1.

$$
Q_{S 1 \_s}=1-\left[\left(1-P_{f S 1}\right)\left(1-P_{f G 1} P_{f G 2}\right)\right]
$$

In a situation where both the nodes and edges are vulnerable to failure, the minimal cut sets for power delivery to $\mathrm{S} 1$ are:

1. $\mathrm{S} 1$

2. G1 and G2

3. G1, T3, and T5

4. G1, T3, and S2 
5. G1, T3, and T4

6. T1 and G2

7. T1, T3, and T5

8. T1, T3, and S2

9. $\mathrm{T} 1, \mathrm{~T} 3$, and $\mathrm{T} 4$

The probability that power will not be delivered to $\mathrm{S} 1$ is then given by:

$$
\begin{aligned}
Q_{S 1}=\left(P_{f S 1}\right) \cup & \left(P_{f G 1} P_{f G 2}\right) \cup\left(P_{f G 1} P_{f T 3} P_{f T 5}\right) \cup\left(P_{f G 1} P_{f T 3} P_{f S 2}\right) \\
& \cup\left(P_{f G 1} P_{f T 3} P_{f T 4}\right) \cup\left(P_{f G 2} P_{f T 1}\right) \cup\left(P_{f T 1} P_{f T 3} P_{f T 5}\right) \\
& \cup\left(P_{f T 1} P_{f T 3} P_{f S 2}\right) \cup\left(P_{f T 1} P_{f T 3} P_{f T 4}\right)
\end{aligned}
$$

where $P_{f S 1}$ and $P_{f S 2}$ are the probabilities of failure of S1 and S2, respectively; $P_{f G 1}$ and $P_{f G 2}$ are the probabilities of failure of G1 and G2, respectively. Using the same steps as before, it can be shown that $Q_{S 1}$ is given by Equation (3.13) considering both node and edge failure.

$$
\begin{aligned}
Q_{S 1}=1-[(1 & \left.-P_{f S 1}\right)\left(1-P_{f G 1} P_{f G 2}\right)\left(1-P_{f G 1} P_{f T 3} P_{f T 5}\right)(1 \\
& \left.-P_{f G 1} P_{f T 3} P_{f S 2}\right)\left(1-P_{f G 1} P_{f T 3} P_{f T 4}\right)\left(1-P_{f G 2} P_{f T 1}\right)(1 \\
& \left.\left.-P_{f T 1} P_{f T 3} P_{f T 5}\right)\left(1-P_{f T 1} P_{f T 3} P_{f S 2}\right)\left(1-P_{f T 1} P_{f T 3} P_{f T 4}\right)\right]
\end{aligned}
$$

Note that in formulating Equations (3.10), (3.11) \& (3.13), it was assumed that the failure of minimal cut sets occurs independently. This is not always true as one component may appear in several minimal cuts. Therefore, system reliability evaluated using the formulated accessibilities (probability of power being delivered) in Equations (3.10), (3.11) \& (3.13), will be the lower bound of the actual reliability. It has been shown that for coherent systems with components that have small probabilities of failure, which is often the case in practice, the lower bound of the reliability is very close to the actual reliability (Esary \& Proschan, 1963). Coherent systems are defined as systems in which (i) if all components are 33 
functioning, the system is functioning, (ii) if all components are in a failed state, then the system failed, and (iii) higher components' reliability implies higher system reliability.

\subsection{Topological-Based System Reliability Formulation}

Given the accessibilities of system components, a single measure of system reliability is required. A simple topological-based approach is to use the weighted reliabilities of system components (Volkanovski et al., 2009). The system reliability is thus given by Equation (3.14).

$$
R_{S}=1-\sum_{i=1}^{N} Q_{i} \frac{C_{i}}{C}
$$

where $Q_{i}$ is the probability that power is not delivered to the $i$ th component (lateral lines or demand substations); $C_{i}$ is the load served by $i$ th component ( $\mathrm{kVA}, \mathrm{kW}$, or number of customers); $C$ is the total load served by the system (kVA, $\mathrm{kW}$, or number of customers); and $N$ is the total number of demand components in the system. $Q_{i}$ as evaluated in the previous section explicitly considers the actual probability of failure of each component in a system.

If detailed information about customer power consumption is available, then $C_{i}$ and $C$ can be defined as the average power supplied by $i$ th component and the average total power supplied by the system, respectively. Doing this will ensure that a line that supplies one customer for example with high power consumption will have a relatively similar effect on system reliability with a line that serves several customers with low power demand. However, due to the varying nature of power consumption, the reliability can only be evaluated using average power consumptions or at a particular time instant. 


\subsection{Line Failure Model}

Failure of distribution and transmission lines here is defined as service failure which is the inability of a line to deliver power to the intended target. Service failure is assumed to occur when the conductor wires are dropped to the ground. For distribution lines, the failure model proposed by Taras et al. (2004) is adopted and is explained here. A distribution line is defined as a switchable section with isolator elements at its ends.

Consider the distribution line shown in Figure 3.7, service failure is defined as the failure of two consecutive poles in a system of three poles. Failure of 2 consecutive poles in any line constitutes service failure regardless of the total number of poles in the line. In the model, a 'system of 3 poles' in a line of $n$ poles is considered because the failure of a central pole, $i$, can cause the failure of either of the adjacent poles $(i+1$ or $i-1)$. The failure of the adjacent poles is conditional on the failure of the central pole.

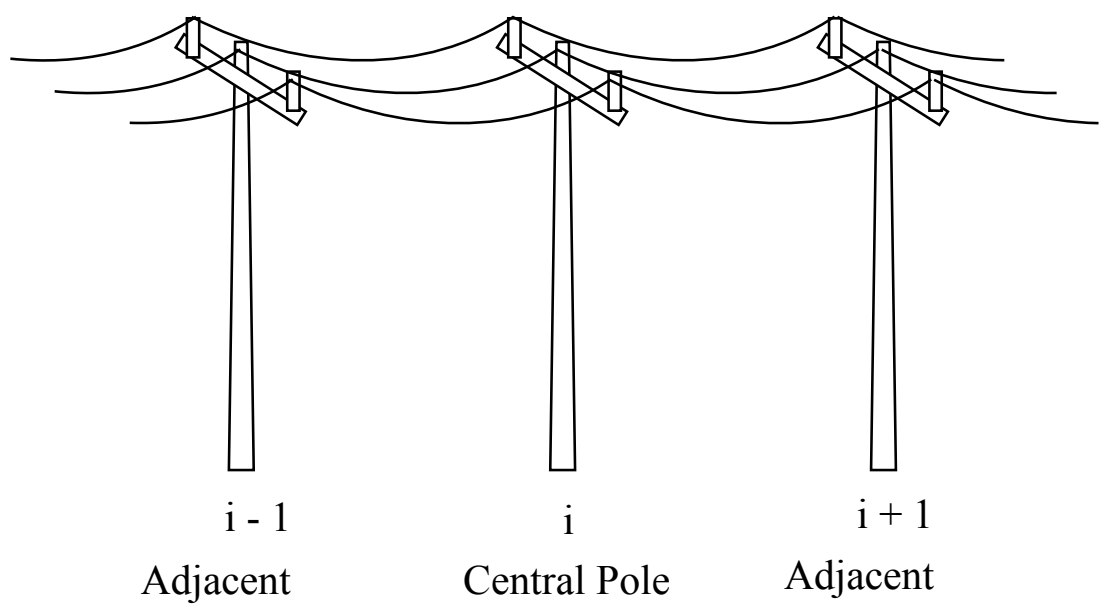

Figure 3.7 Distribution line model 
To determine the probability of service failure in a system of three poles, the following are defined Taras et al. (2004):

- Probability of service failure of a system with three poles is defined as $P_{I}$

- Probability of failure of the central pole " $i$ " is defined by $P\left(F_{i}\right) \equiv P_{i}$

- Probability of failure of an adjacent pole conditional on the failure of the central pole is defined by $P\left(F_{i-1} \mid F_{i}\right)=P\left(F_{i+1} \mid F_{i}\right) \equiv P_{a}$

- The conditional probability of failure of the adjacent poles is evaluated by increasing their applied load by $50 \%$ to account for load sharing after the failure of the central pole. Note that increasing the load means increasing the wind pressure area or the ground line moment, rather than increasing the wind speed.

The probability of service failure in the system with three poles is:

$$
\begin{gathered}
P_{I}=P\left(F_{i}\right) \cdot P\left[\left(F_{i-1} \cup F_{i+1}\right) \mid F_{i}\right] \\
P_{I}=P\left(F_{i}\right) \cdot\left\{P\left[F_{i-1} \mid F_{i}\right]+P\left[F_{i+1} \mid F_{i}\right]-P\left[\left(F_{i-1} \cap F_{i+1}\right) \mid F_{i}\right]\right\}
\end{gathered}
$$

Assuming that the failures of the adjacent poles are independent of each other, then

$$
P_{I}=P\left(F_{i}\right) \cdot\left\{P\left[F_{i-1} \mid F_{i}\right]+P\left[F_{i+1} \mid F_{i}\right]-P\left[\left(F_{i-1} \mid F_{i}\right)\right] \cdot P\left[\left(F_{i+1} \mid F_{i}\right)\right]\right\}
$$

The above equation can be rewritten as:

$$
P_{I}=P_{i} \cdot\left\{2 P_{a}-P_{a}^{2}\right\}
$$

Equation (3.18) is applied to each pole along a line. The number of all possible failure modes in a line is the same as the number of poles in the line. Assuming all failure modes are fully independent, the overall failure probability of an entire line is given by Equation 
(3.19). This is also the upper bound of the probability of failure. If all the failure modes are fully dependent, the probability of failure is the lower bound given by Equation (3.20). Note that the exact failure probability of a line lies between the given bounds. Determination of the exact failure probability requires a knowledge of the correlation coefficient between poles in a line which can vary depending on the location of each pole relative to a failed pole. In subsequent life cycle cost analysis in this study, the upper bound of the probability of failure of the lines is used so as to be conservative.

$$
\begin{gathered}
\text { Upper bound: } \quad P_{f L}=1-\prod_{i=1}^{m}\left[1-P_{I}\right] \\
\text { Lower bound: } \quad P_{f L}=\max \left[P_{I}\right]
\end{gathered}
$$

where $P_{f L}$ is the probability of failure of an entire line and $m$ is the total number of poles in the line. Using the above concept, the probabilities of failure of the lines in a power system can be calculated. The reliability of a line is the complement of the probability of failure.

Transmission line ends are assumed to be between two substations, between a substation and a branching point, or between any two branching points. The span between support structures of transmission lines is usually long enough that the failure of one support structure will lead to service failure of the line. This is unlike in distribution system where the spans are relatively short and the failure of one pole may not necessarily lead to service failure of the line. If a transmission line is modeled as a series system, the lower bound of the probability of failure of the line is obtained by assuming that the failure modes are fully dependent. The upper bound, on the other hand, is obtained by assuming that the failure modes are fully independent. The lower and upper bounds of the probability of failure are given by Equation (3.21). 


$$
\max \left[P_{S}\right] \leq P_{f L} \leq 1-\prod_{i=1}^{N}\left[1-P_{S}\right]
$$

where $P_{f L}$ is the probability of failure of the line; $P_{S}$ is the probability of failure of a single support structure; and $N$ is the total number of support structures in the line. In this research, full independence is assumed between the modes and hence the upper limit in Equation (3.21) is used for evaluating transmission line failure. This is reasonable due to the long span between structures. 


\section{Targeted Hardening of Distribution Systems Subjected to Hurricanes $^{1}$}

\subsection{Introduction}

Over the years, several methods of hardening the distribution system have been studied. One of the methods studied extensively is undergrounding the system (e.g. Francis et al. (2011), Brown (2009), Xu and Brown (2008b), CVSCC (2004), FPSC (2005), LIPA (2005), TDA (2006)). However, most of these studies concluded that undergrounding is not cost-effective. Another method being currently studied is targeted hardening of current overhead distribution systems. Targeted hardening involves strengthening important support structures as well as structures with very high probability of failure. Important structures include distribution poles that serve a large number of customers, poles that serve critical customers (hospitals, fire stations, police stations, economic centers) and poles that are difficult to access. Brown (2009) studied the hardening of $10 \%$ of distribution poles in Texas and estimated the net benefit derived from it. Bjarnadottir et al. (2014) studied targeted hardening of distribution poles in Florida by replacing poles that fail with poles that are one class higher.

The above studies did not attempt to identify risk-critical parts of the system to be strengthened or evaluate the effect of the targeted hardening on overall system reliability. To determine the critical parts or components of a system, some form of component importance measure is required. This, in turn, requires evaluating the reliability of the whole system. However, previous studies (e.g. Han et al. (2013), Shafieezadeh et al. (2014a), Bjarnadottir et al. (2013), Ryan et al. (2014b)) conducted on the vulnerability of distribution systems to hurricane and extreme wind damage focuses on evaluating the reliabilities of individual poles rather than the whole system.

\footnotetext{
${ }^{1}$ A version of this chapter was previously published in Reliability Engineering \& System Safety and is reused herein with permission from Elsevier. The permission is presented in Appendix B.
} 
This chapter presents and demonstrates a framework that can be used to evaluate the effectiveness of targeted hardening measures. The framework includes fragility analysis considering decay of poles, hurricane hazard analysis, system reliability evaluation, component importance measure, and cost analysis. The flow chart of the general framework is shown in Figure 4.1. The framework is explained and demonstrated at the same time using a notional power distribution system. 


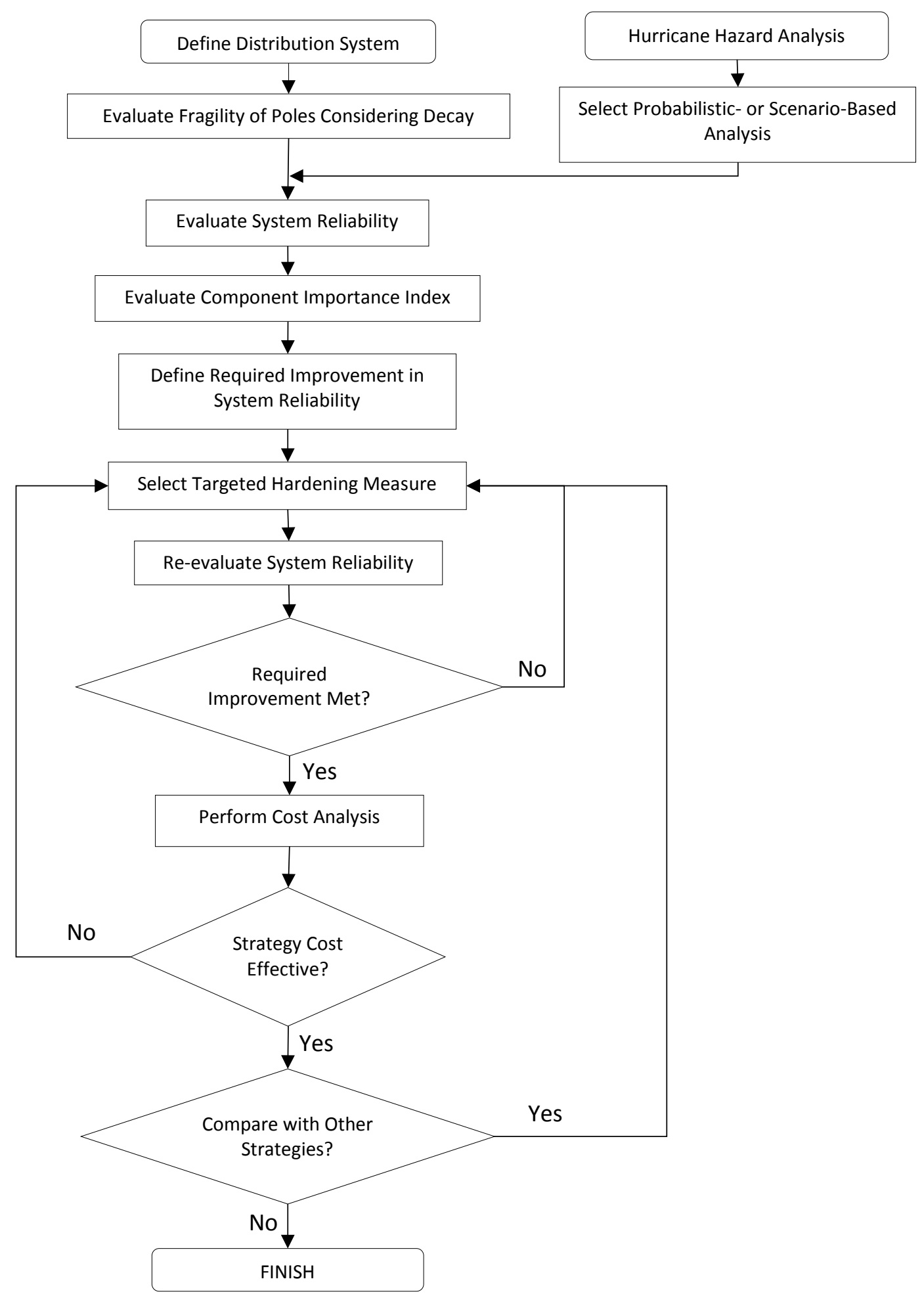

Figure 4.1 Flowchart of proposed framework 


\subsection{Power Distribution System Model}

The power system model adopted for demonstrating the framework is shown in Figure 4.2. It is the power system of a virtual city called "Micropolis" developed at Texas A\&M University for use in infrastructure risk research and planning (Brumbelow et al., 2007; Bagchi, 2009; Bagchi et al., 2009). The city has approximately 5000 residents in a historically rural region. The city is assumed to be located on the east coast of Florida, with the middle of the city located at $27.6^{\circ} \mathrm{N}$ and $80.4^{\circ} \mathrm{W}$. The city has one substation supplied by a sub-transmission line (138 $\mathrm{kV}$ rating) running through the city. Two three-phase feeders emanate from the substation to deliver power to the entire city by branching off to smaller three-phase sub-branches and single-phase laterals. The dots in Figure 4.2 represent the poles while the conductor wires are represented by solid lines. Most of the left side of the city is served by an underground system. However, in this research, the underground system is transformed to an overhead system so that the entire system can be considered.

Figure 4.2 only shows poles carrying distribution transformers and directly serving customers. However, the city is approximately 2 miles by 1 mile. Therefore, there are a lot more poles in the city than shown in Figure 4.2. The number of poles in each line is found based on the span of the poles which is taken as $46 \mathrm{~m}$ as will be discussed subsequently. The total number of poles in the city is approximately 661. The total circuit line is approximately $30.3 \mathrm{~km}$. There are an estimated 434 residential, 15 industrial, and 9 commercial/institutional customers in the city including 3 schools and 3 churches (Brumbelow et al., 2007). Figure 4.3 shows the line diagram of the power distribution system. The system is assumed to be radially operated at all times. Radially operated implies there is a unique path from the source of power to each component or customer. 


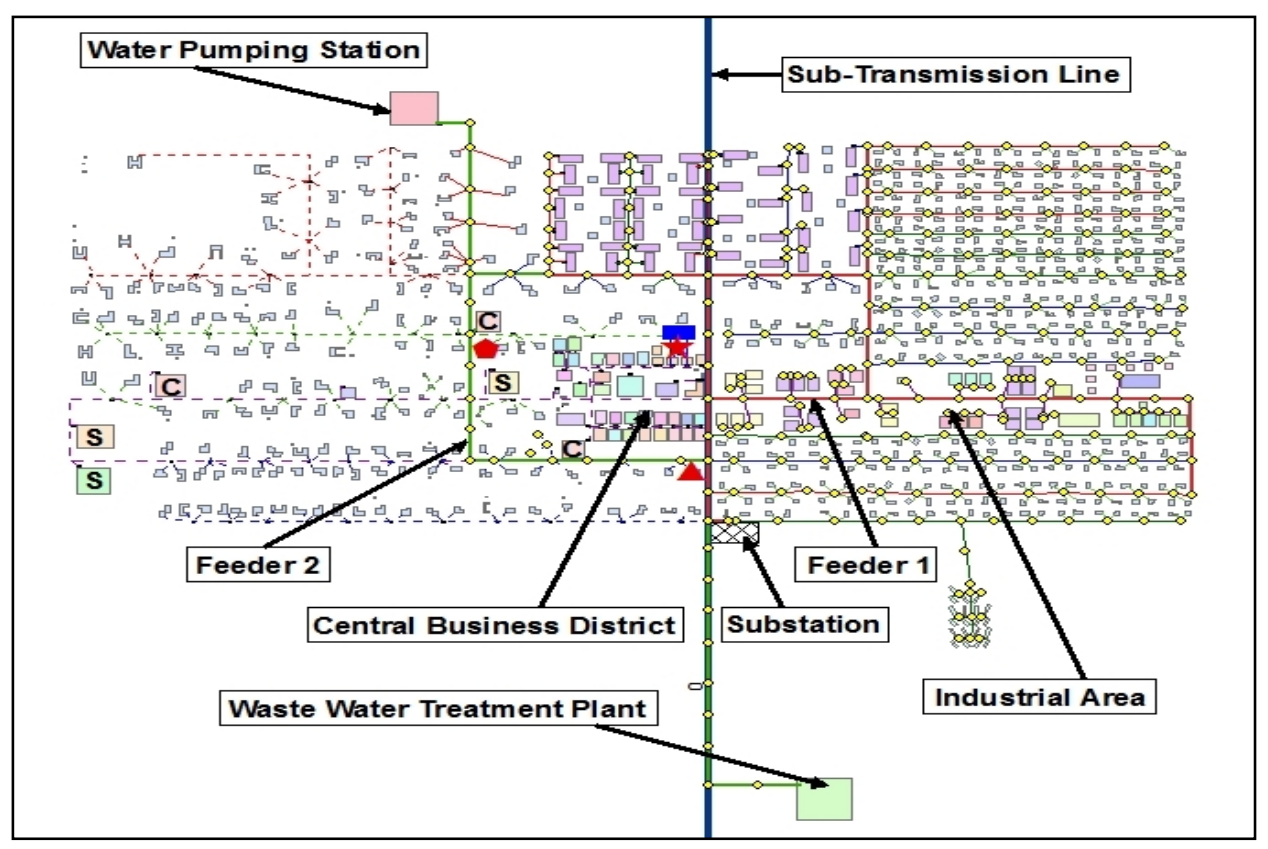

Figure 4.2 Micropolis power distribution system

[C IEEE. Reprinted, with permission, from Bagchi et al. (2009). Modeling the impact of fire spread on the electrical distribution network of a virtual city. Paper presented at the North American Power Symposium (NAPS), 2009. See Appendix C]

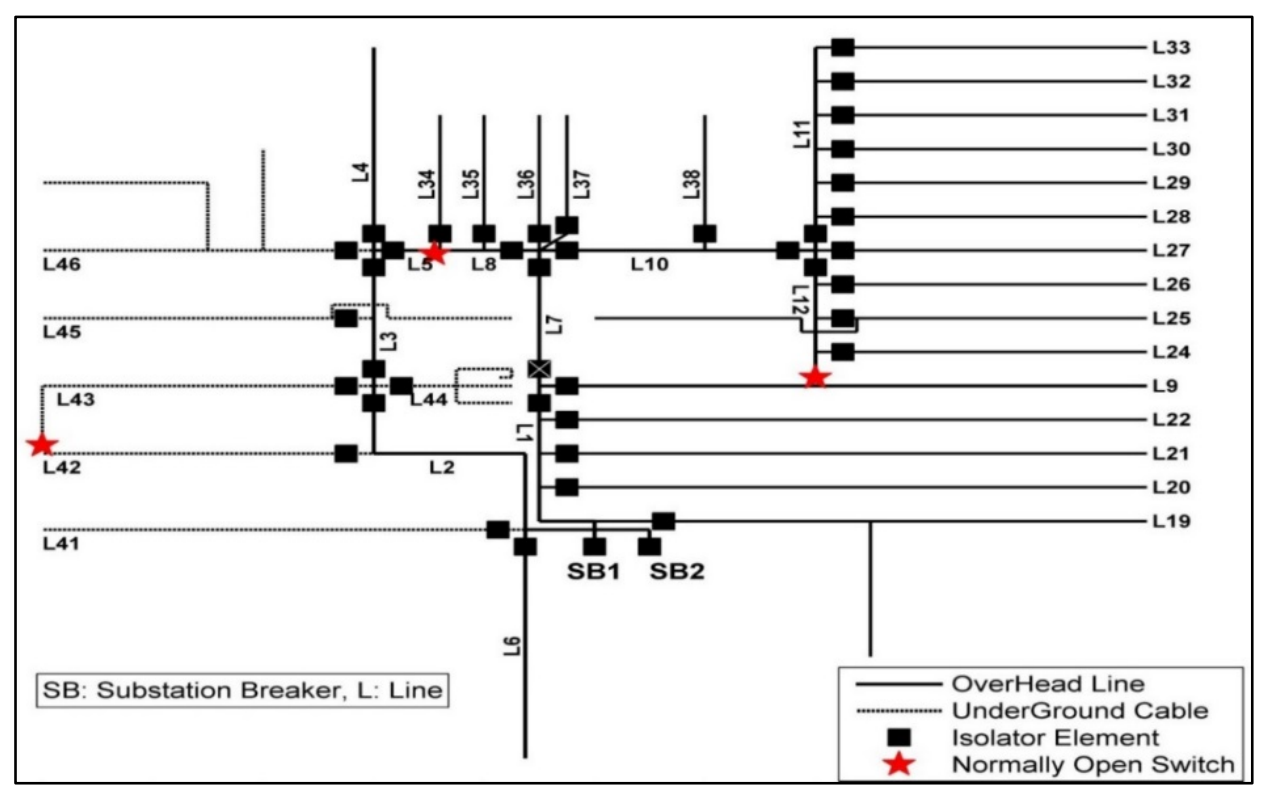

Figure 4.3 Micropolis power distribution system line diagram

[C IEEE. Reprinted, with permission, from Bagchi et al. (2009). Modeling the impact of fire spread on the electrical distribution network of a virtual city. Paper presented at the North American Power Symposium (NAPS), 2009. See Appendix C] 


\subsection{Design of Poles}

The poles supporting the distribution lines are assumed to be southern pine wood poles as they are the dominant used in the U.S. (Gustavsen \& Rolfseng, 2000; Wolfe \& Moody, 1997). The poles are designed using the reliability-based method recommended by ASCE111 (2006). Figure 4.4 shows the distribution poles layout. A typical distribution pole that is $13.7 \mathrm{~m}$ high is considered. The three-phase main feeder poles are assumed to support three Aluminum Conductor Steel Reinforced (ACSR) conductor wires with diameters of $18.3 \mathrm{~mm}$. They are also assumed to support one all-aluminum conductor (AAC) neutral wire with a diameter of $11.8 \mathrm{~mm}$. The single-phase laterals are assumed to support two ACSR conductor wires and one AAC neutral wire. All the poles are assumed to have a span of $46 \mathrm{~m}$ for wind pressure calculations (Short, 2006).

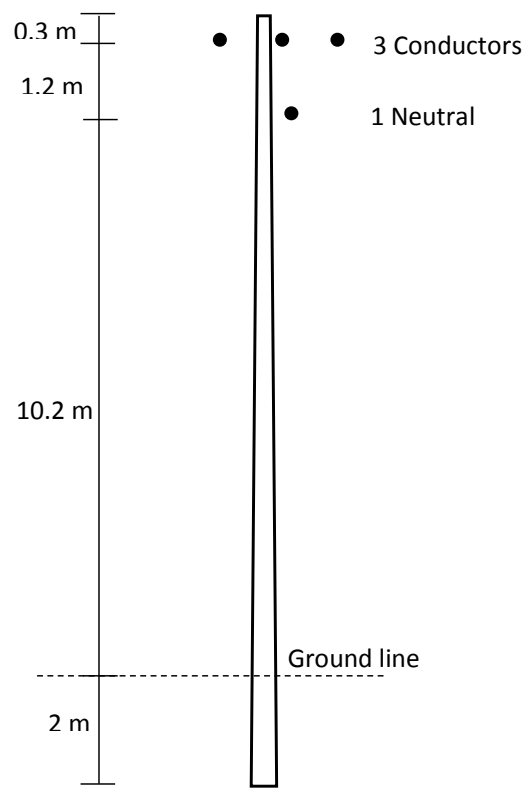

(a)

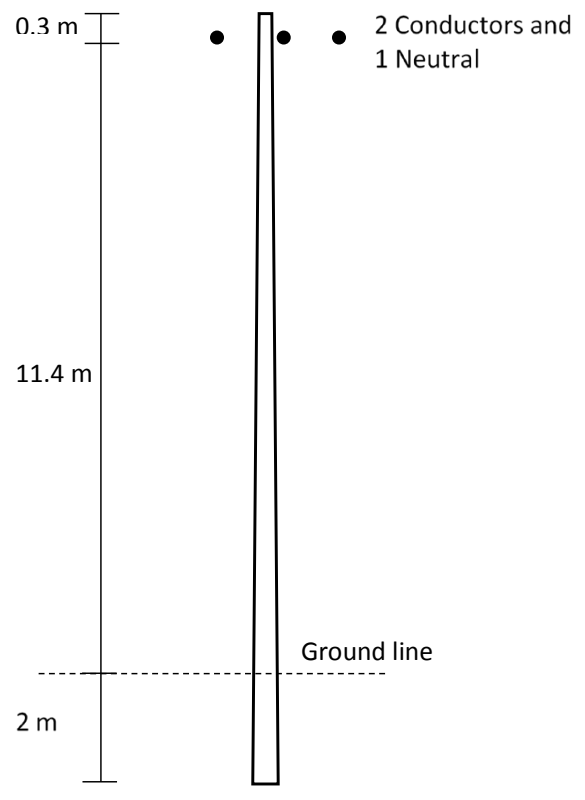

(b)

Figure 4.4 Distribution poles layout

(a) three-phase line poles (b) single-phase line poles 
The poles are designed for NESC (2002) grade C construction for weather-related loads which are often the controlling conditions (ASCE-111, 2006). The design equation that controls reliability of weather-related events is given by (ASCE-111, 2006):

$$
\phi R_{n}>\text { effect of }\left[1.1 D L \text { and } \gamma Q_{50}\right]
$$

where $\phi$ is strength factor, $R_{n}$ is the nominal strength, $D L$ is dead loads, $\gamma$ is load factor, and $Q_{50}$ is 50-year return period wind load. The design 50-year return period 3-sec gust wind speed for the chosen location is about $52 \mathrm{~m} / \mathrm{s}$ based on ASCE-7 (2010). Based on the wind speed, the wind force acting on the pole and the wires can be calculated using Equation (4.2) from ASCE-74 (1991). ASCE-111 (2006) recommends the use of this equation for both transmission and distribution support structures.

$$
F=Q K_{z} K_{z t}(V)^{2} G C_{f} A
$$

where $F$ is force $(\mathrm{N}), Q$ is air density factor, $K_{z}$ is exposure coefficient, $V$ is basic 3 -sec gust wind speed, $G$ is gust response factor, $C_{f}$ is force or drag coefficient, $K_{z t}$ is topographic factor, and $A$ is the area projected on a plane normal to the wind direction $\left(\mathrm{m}^{2}\right)$. To account for P- $\Delta$ effect, ASCE-111 (2006) recommends using the method developed by Gere and Carter (1962). The method involves determining an amplification factor to account for the P- $\Delta$ effect. The amplification factor is given by Equation (4.3).

$$
\text { Amplication factor }=\frac{1}{1-\left(\frac{V L}{P_{c r}}\right)}
$$

where $V L$ is total factored vertical load, $P_{c r}$ is buckling load given by Equation (4.4).

$$
P_{c r}=\left(\frac{\pi^{2} \times M O E \times I_{\text {top }}}{[2 L]^{2} \times 144}\right)\left(\frac{D_{\text {bottom }}}{D_{\text {top }}}\right)^{2.7}
$$


where $M O E$ is the mean modulus of elasticity, $I_{t o p}$ is the moment of inertia at top of pole, $L$ is the distance from the ground line to centroid of horizontal loads, $D_{b o t t o m}$ and $D_{t o p}$ are the bottom and top diameters, respectively. Class 4 and class 5 southern pine poles are initially assumed to be sufficient for the three-phase and single-phase lines respectively. The values of the variables in Equation 4.2 are given in Table 4.1 while the parameters related to the poles are given in Table 4.2. Note that the initial strength and geometry of the poles are found in ANSI-O5.1 (2002). Comparing the required and actually ground line circumferences from Table 4.2, it can be seen that the class 4 and class 5 southern pine poles are adequate for the three-phase and single-phase lines, respectively as assumed.

Table 4.1 Design variables

\begin{tabular}{lll}
\hline \hline variable & Mean values for pole & Mean values for wires \\
\hline $\mathrm{G}$ & 0.948 & 0.85 \\
$\mathrm{C}_{\mathrm{f}}$ & 0.9 & 1.0 \\
$\mathrm{~K}_{\mathrm{z}}$ & 0.951 & 1.029 \\
$\mathrm{Q}$ & 0.613 & 0.613 \\
$\mathrm{~K}_{\mathrm{zt}}$ & 1.0 & 1.0 \\
$\mathrm{~A}\left(\mathrm{~m}^{2}\right)$ & 2.644 for class 4 and 2.439 for 0.842 per conductor \\
& class 5 & and 0.543 for neutral \\
\hline \hline
\end{tabular}


Table 4.2 Poles parameters

\begin{tabular}{lll}
\hline \hline Parameter & $\begin{array}{l}\text { Three-phase } \\
\text { poles }\end{array}$ & $\begin{array}{l}\text { Single-phase } \\
\text { poles }\end{array}$ \\
\hline Strength, $R_{n}(\mathrm{MPa})$ & 55.2 & 55.2 \\
Strength factor, $\phi$ & 0.79 & 0.79 \\
Top circumference $(\mathrm{m})$ & 0.53 & 0.48 \\
Circumference at $1.8 \mathrm{~m}$ from butt $(\mathrm{m})$ & 0.89 & 0.83 \\
Total ground line moment $(\mathrm{N}-\mathrm{m})$ & 69,212 & 55,760 \\
Amplification factor & 1.1 & 1.1 \\
Design ground line moment $(\mathrm{N}-\mathrm{m})(\mathrm{Amp}$ factor * total GLM) & 76,133 & 61,336 \\
Required section modulus $\left(\mathrm{m}^{3}\right)$ & 0.00175 & 0.00141 \\
Required circumference at ground line $(\mathrm{m})$ & 0.82 & 0.763 \\
Actual circumference at ground line $(\mathrm{m})$ & 0.884 & 0.824 \\
\hline \hline
\end{tabular}

\subsection{Decay of Wood Poles}

Wood poles are susceptible to decay due to fungal attack and are also vulnerable to attack by insects and woodpeckers. Decay usually occurs at the ground level or just below the ground. The rate of decay of wood depends on several factors such as timber species, climatic conditions (temperature, rainfall, and humidity), initial preservative treatment, and nature of fungal/insect attack. This means that any decay model can only be an approximation. The decay model from Li et al. (2005) and Shafieezadeh et al. (2014b) is adopted in this research. The strength of poles as a function of time is given by Equation (4.5).

$$
R(t)=R_{o}\left[1-\min \left(\max \left(a_{1} t-a_{2}, 0\right), 1\right) \times \min \left(\max \left(b_{1} t^{b_{2}}, 0\right), 1\right)\right]
$$

where $R(t)$ is the strength at time $t, R_{o}$ is the initial strength. The values of $a_{1}, a_{2}, b_{1}$, and $b_{2}$ were found from regression analysis as $0.014418,0.10683,1.3 \times 10^{-4}$, and 1.846 
respectively by Li et al. (2005) and Shafieezadeh et al. (2014b). Figure 4.5 shows the residual strength remaining as a function of time for southern pine poles calculated using Equation (4.5). According to the NESC (2002), wood poles should be replaced or reinforced when their strength falls below $67 \%$ of the initial strength. From Figure 4.5 , this will happen when the poles are around 73 years old. The long service life of the poles from the decay model might be because the poles used to develop the model are in-service poles that have undergone periodic maintenance.

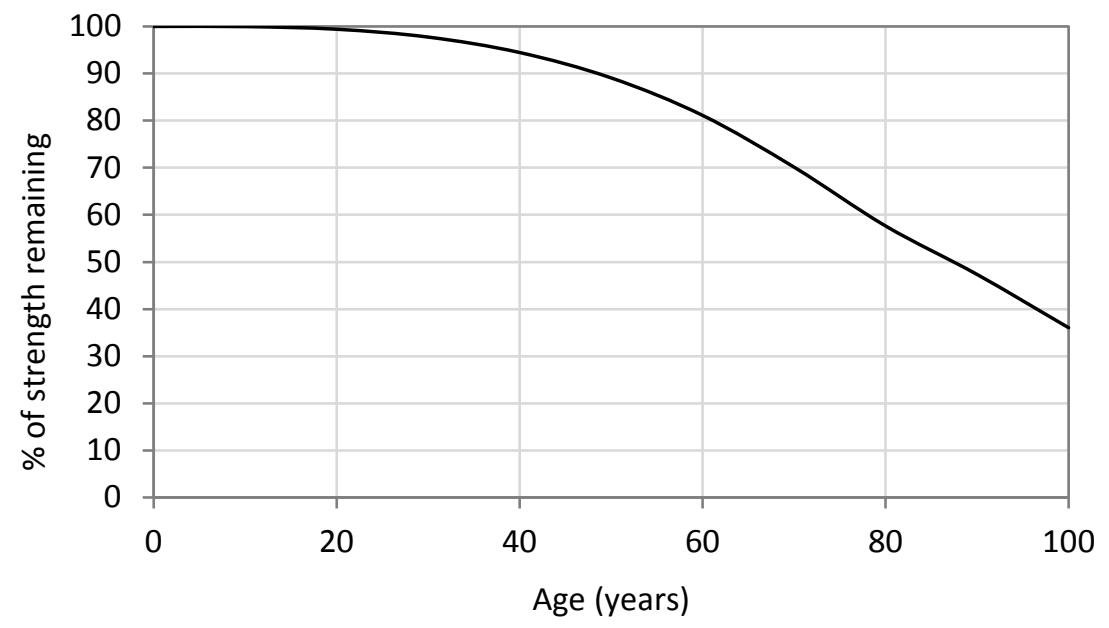

Figure 4.5 Residual strength of poles as a function of time

Shafieezadeh et al. (2014b) also used the data from Li et al. (2005) to plot the variation of c.o.v of southern pine poles with age. The plot showed that as the poles age, the uncertainty in the strength increases. The figure developed by Shafieezadeh et al. (2014b) is used to obtain the c.o.v of the pole strength at different ages in this study. The above decay model has shortcomings because it does not incorporate variation in soil properties as well as variation in decay rate due to wood specie. Due to these shortcomings, location-specific models can be developed by utility companies to obtain more accurate results. 


\subsection{Fragility Analysis}

Fragility analysis is required to calculate the probability of failure of the power lines. Fragility is the probability of failure of a structure subjected to a given load. Monte Carlo simulation is used to calculate the probabilities of failure of the poles while varying the basic 3-sec gust wind speed. For each random variable with uncertainty, 1,000,000 random values were generated. Only flexural failure due to wind load is considered in the analysis. It is acknowledged that other failure mechanisms such as foundation failure and failure caused by falling trees and flying debris are also important in practice. However, there is a lack of data to include these failure mechanisms in the structural reliability formulation. If such data become available, these failure mechanisms can be easily included.

The probabilities of failure were calculated by counting the number of cases where the stress demand at the ground line exceeds the corresponding stress capacity. Even though the poles are tapered, the critical stress for short poles, such as the ones normally used in

distribution systems, is commonly at the ground line (ASCE-111, 2006). The limit state function for the fragility analysis is given by:

$$
G(t)=R(t)-S
$$

where $R(t)$ is the strength of the poles at any time $t$ and $S$ is the stress demand at the ground line caused by wind speed. The steps of the fragility analysis are summarized in the flow chart of Figure 4.6. 


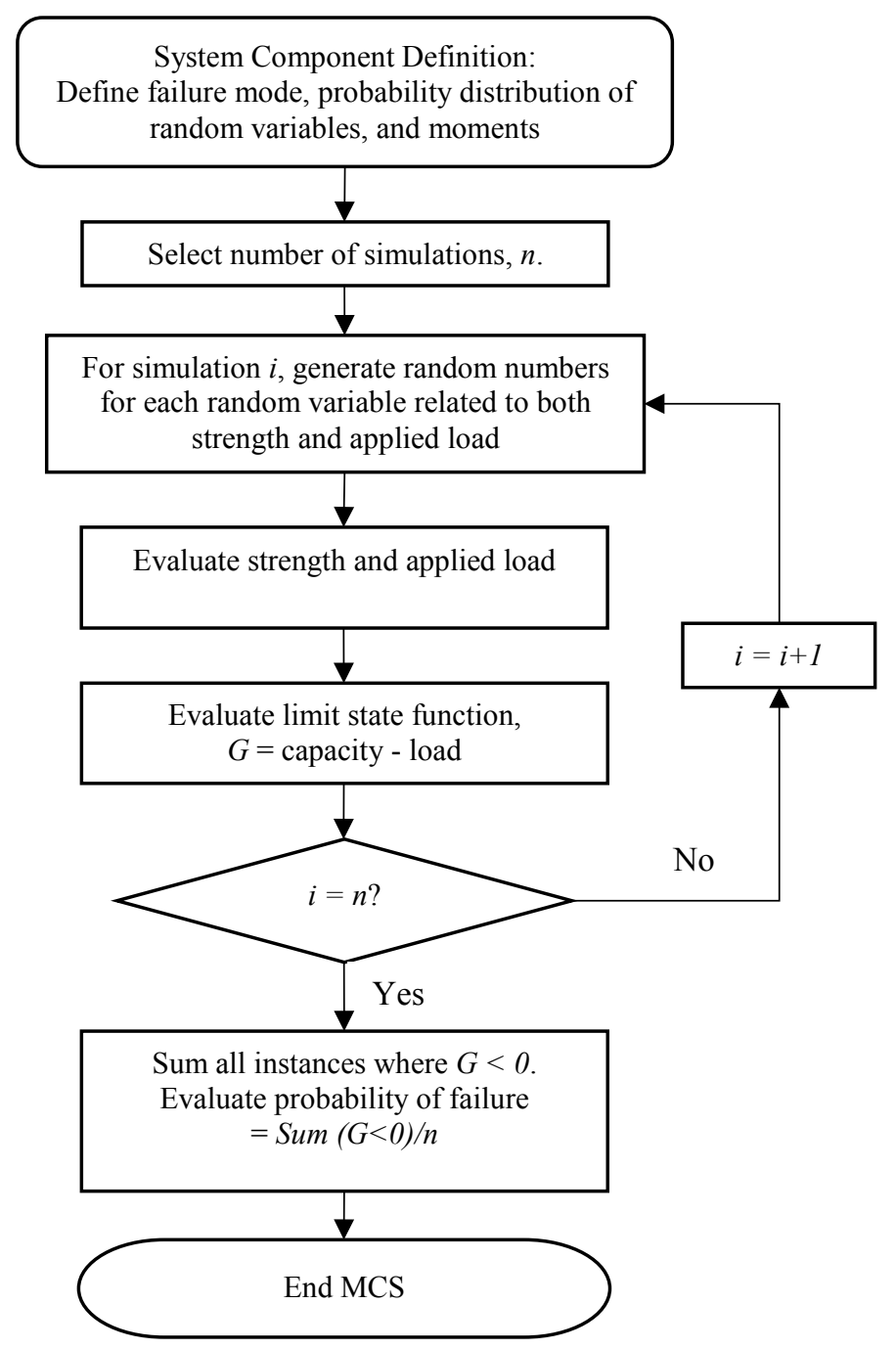

Figure 4.6 Fragility analysis flowchart

The initial strength of the poles is determined from ANSI-O5.1 (2002) while the strength as the system ages is determined by considering decay of the poles as described previously. The stress due to applied wind load is determined using the force from Equation ((4.2). The uncertain parameters related to the pole strength and applied stress and their c.o.v are summarized in Table 4.3 . 
Table 4.3 Strength and load parameters and their statistics

\begin{tabular}{llll}
\hline Random variable & $\begin{array}{l}\text { Probability } \\
\text { distribution }\end{array}$ & c.o.v & Source \\
\hline Fiber Strength $(\mathrm{kPa})$ & Lognormal & Varies with time & Shafieezadeh et al. (2014b) \\
Pole Height above ground $(\mathrm{m})$ & Normal & 0.03 & Assumed \\
Wind Area $\left(\mathrm{m}^{2}\right)$ & Normal & 0.06 & Wolfe and Moody (1997) \\
$\mathrm{G}$ & Normal & 0.11 & Ellingwood and Tekie (1999) \\
$\mathrm{C}_{\mathrm{f}}$ & Normal & 0.12 & Ellingwood and Tekie (1999) \\
$\mathrm{K}_{\mathrm{z}}$ & Normal & 0.06 & ASCE-111 (2006) \\
\hline \hline
\end{tabular}

The fragility curves of the poles at different ages are shown in Figure 4.7 and Figure 4.8. It can be seen that because of decay, the probabilities of failure given wind speed increases as the poles age. It can also be seen that compared to the three-phase poles, the singlephase poles have slightly lower probabilities of failure even though they have a slightly smaller ground line diameter. This is because the single-phase poles carry fewer conductors as shown in Figure 4.4.

Overhead lateral lines use pole-mounted distribution transformers (DTs) to serve customers. Typical 25kVA rated DTs are assumed to serve most of the customers in Micropolis and each DT is assumed to serve an average of four customers (Gonen, 2014). It is acknowledged that higher rated transformers are required for few of the customers in the city such as churches and industrial buildings. For a lateral line like line 20 in Figure 4.2 and Figure 4.3 which serves 48 residential customers and is about $1.4 \mathrm{~km}$ long, it has 12 poles carrying DTs out of a total of about 30 poles. Based on information from commercially available pole mounted DTs, the DTs on the poles are assumed to be $0.39 \mathrm{~m}$ in diameter, $0.61 \mathrm{~m}$ in cylinder height, and weighing about $160 \mathrm{~kg}$.

Fragility curves of new and 60-year-old single-phase poles carrying DTs are shown in Figure 4.8. It can be seen that the presence of DTs slightly increases the probabilities of failure at different wind speeds. For example, at a wind speed of $60 \mathrm{~m} / \mathrm{s}$, new lateral line poles without DTs have a probability of failure of 0.19 compared to 0.22 for new poles 
with DTs. For poles that are 60 years old, the probability of failure at $60 \mathrm{~m} / \mathrm{s}$ with and without DTs is 0.56 and 0.60 , respectively.

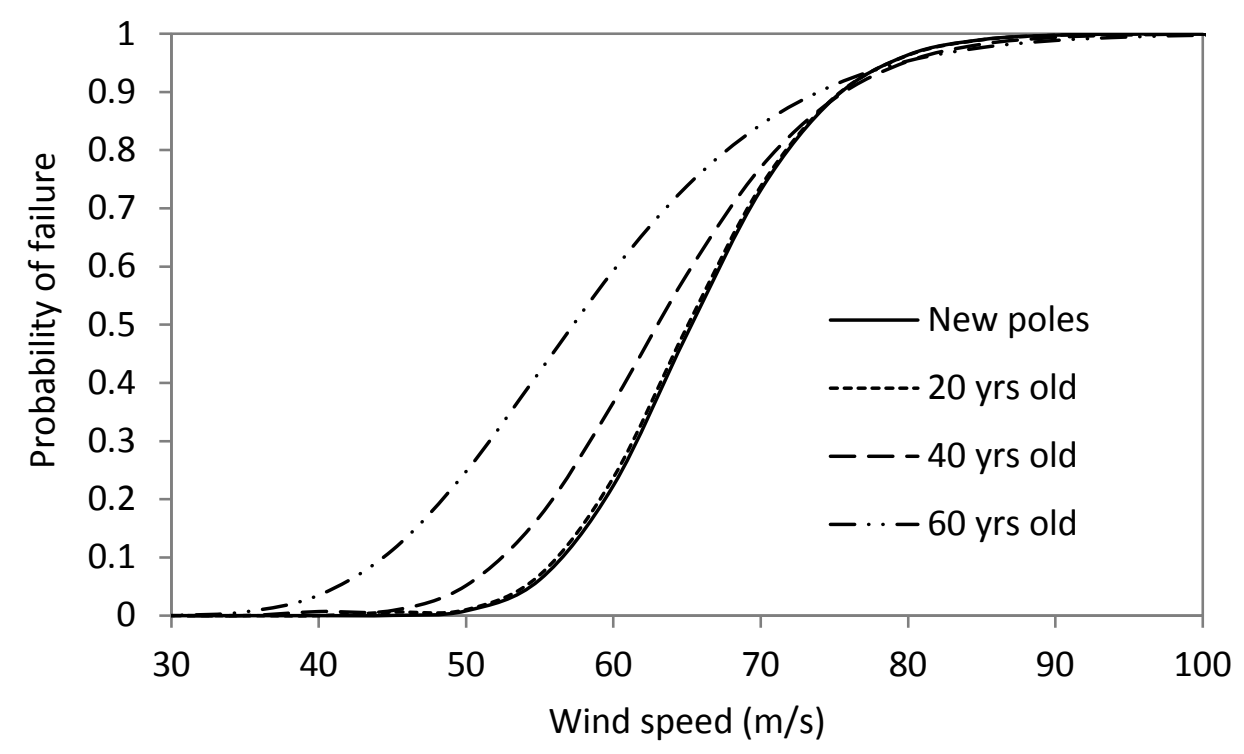

Figure 4.7 Fragility curves of three-phase line poles at 0, 20, 40, and 60 years

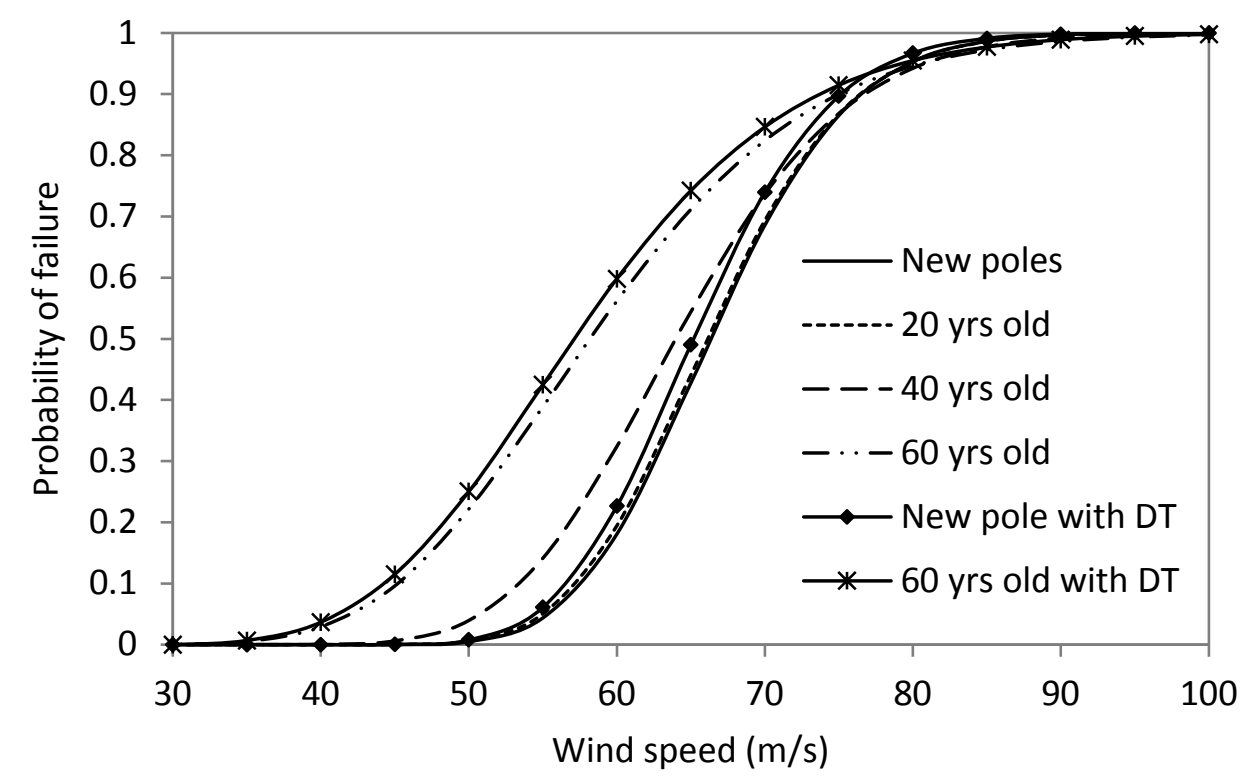

Figure 4.8 Fragility curves of single-phase line poles at 0, 20, 40, and 60 years 
The lognormal distribution is assumed to describe the fragility models as recommended by Bjarnadottir et al. (2013). A Kolmogorov-Smirnov test was carried out to confirm this assumption. The lognormal CDF is given by:

$$
F_{R}(v)=\Phi\left[\frac{\ln (v / m)}{\zeta}\right]
$$

where $m$ is the median of the fragility function, and $\zeta$ is the logarithmic standard deviation of intensity measure.

\subsection{Hurricane Risk Assessment}

There are two ways to assess hurricane risk (Li, 2012). The first method is probabilistic hurricane analysis where historical hurricane records are used to develop probability density function for key hurricane parameters such as the location of origin, translation speed, heading angle, central pressure, and radius to maximum wind location. Monte Carlo simulation is then performed to simulate future hurricanes which can be used to estimate maximum wind speeds as demonstrated by Vickery et al. (2000b). The wind speed from the above simulation is then modeled as a random variable and convolved with fragility model to assess the risk of structures or components as demonstrated by Li and Ellingwood (2006).

The second method is a scenario-based approach where the effect of a specific simulated or historical hurricane is studied rather than the aggregated effect of all possible hurricanes as in the previous approach. Another difference between the two methods is that probabilistic analysis considers the probability of occurrence of different levels of hurricanes while scenario-based analysis assumes a selected level of hurricane (e.g. 200year return period hurricane or category 4 hurricane). 


\subsubsection{Probabilistic Analysis}

Using the probabilistic analysis, the annual probability of failure of the poles is estimated by convolving the structural fragility with a hurricane wind speed model as proposed by $\mathrm{Li}$ and Ellingwood (2006). The annual probability of failure is given by:

$$
P_{f}=\int_{0}^{\infty} F_{R}(v) f_{v}(v) d v
$$

where $F_{R}(v)$ is the cumulative distribution function (CDF) of the structural fragility and $f_{v}(v)$ is the probability density function (PDF) of the hurricane wind speed. Vickery et al. (2000b) conducted simulations of hurricanes and proposed that the Weibull distribution is appropriate for hurricane wind speed prediction. The PDF of the Weibull equation is given by:

$$
f_{v}(v)=\frac{\alpha}{u}\left(\frac{v}{u}\right)^{\alpha-1} \exp \left[-\left(\frac{v}{u}\right)^{\alpha}\right]
$$

where $v$ is the wind speed, and $u$ and $\alpha$ are the parameters of the Weibull distribution. The wind speed $v$, is related to the return period $(T)$ of the hurricane by:

$$
v=u\left[-\ln \left(\frac{1}{T}\right)\right]^{\frac{1}{\alpha}}
$$

The 50, 100, 250, and 500-year return period 3-s gust wind speeds for the chosen location are estimated to be 56, 60, 67, and $72 \mathrm{~m} / \mathrm{s}$, respectively from Vickery et al. (2009b). The Weibull parameters are then calculated using Equation (4.10) as $u=26.7$ and $\alpha=1.85$. The annual probabilities of failure of the poles considering decay over time are calculated and plotted in Figure 4.9. It can be seen from the figure that the annual probabilities of failure increase with age due to decay of the poles. It can also be seen that the presence of 
distribution transformers (DTs) has little effect on the annual probability of failure of the single-phase poles. For subsequent analysis, the fragility results of lateral line poles without DTs will be used as they are the dominant network vulnerability.

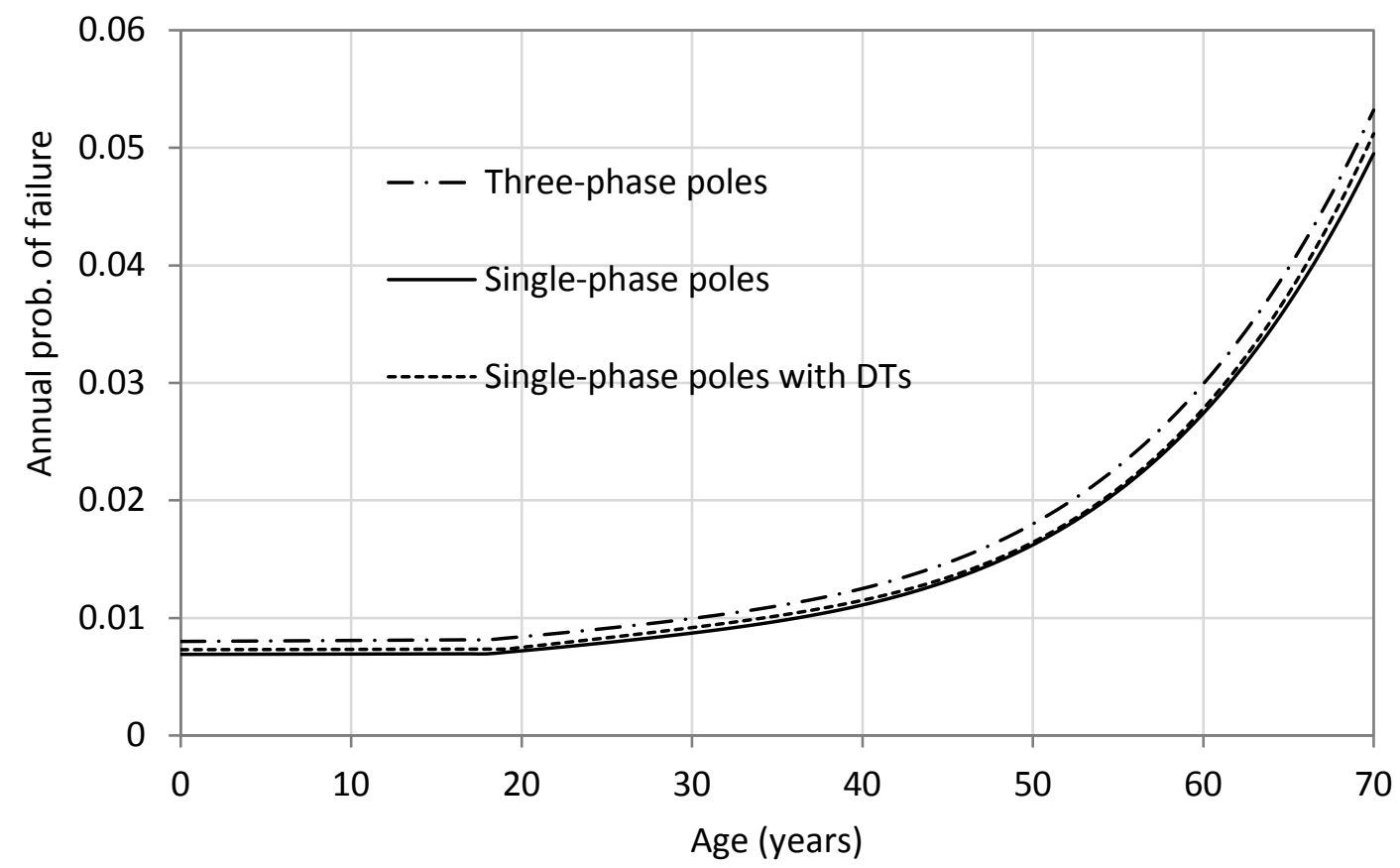

Figure 4.9 Annual probability of failure with age

\subsubsection{Scenario-Based Analysis}

Hurricane Jeanne has been selected for demonstrating the proposed framework. Hurricane Jeanne became a tropical storm on 14 September 2004 and made landfall on the east coast of Florida on 26 September as a category 3 hurricane (Lawrence \& Cobb, 2005). Hurricane track data and recorded wind speeds are obtained from the North Atlantic Hurricane Database (HURDAT) from the National Oceanic and Atmospheric Administration (NOAA, 2015b) website. The database provides the location (latitude and longitude) as well as the maximum wind speed and central pressure of the hurricane mostly at 6-hour 
intervals. However, accurate analysis of a relatively small area such as the study area, in this case, cannot be done considering 6-hour intervals as the hurricane can cover a long distance within this period.

To obtain finer records of the hurricane wind speeds and central pressure, the 6-hour records are interpolated linearly to obtain 30-minute interval records. Linear interpolation has been shown to produce accurate results (Jayaram \& Baker, 2010). At every time instant, the wind speed at any location from the hurricane eye can be determined using the windfield model developed by Holland (1980) as:

$$
V_{G}=\left[\left(\frac{R_{\max }}{r}\right)^{B}\left(\frac{B \Delta p \exp \left[-\left(\frac{R_{\max }}{r}\right)^{B}\right]}{\rho}\right)+\frac{r^{2} f^{2}}{4}\right]^{1 / 2}-\frac{r f}{2}
$$

where $R_{\max }$ is the radius to maximum wind speed, $r$ is the distance from hurricane eye to point of interest, determined using the Haversine formula, $B$ is the Holland parameter, $\Delta p$ is the central pressure difference, $\rho$ is air density $\left(1.15 \mathrm{~kg} / \mathrm{m}^{3}\right)$, and $f$ is the Coriolis parameter. $R_{\max }, B$, and $f$ are given by (FEMA, 2011; Powell et al., 2005):

$$
\begin{gathered}
\ln R_{\text {max. }}=2.556-0.000050255 \Delta p^{2}+0.042243032 \psi \\
B=1.881-0.00557 R_{\text {max }}-0.010917 \psi, \quad \sigma_{B}=0.286 \\
f=2 \Omega \sin \varphi
\end{gathered}
$$

where $\psi$ is the storm latitude, $\Delta p$ is the central pressure difference, $\Omega$ is the earth's angular velocity $\left(7.292 \times 10^{-5} \mathrm{rad} / \mathrm{s}\right)$, and $\varphi$ is the local latitude. The HURDAT data provides the central pressure for the hurricanes at each time step. The central pressure difference, $\Delta p$, is found by subtracting the central pressure from atmospheric pressure at a distance beyond the effect of the hurricane which is typically 1,013 millibars (Xu \& Brown, 2008b; Wang \& Rosowsky, 2012). 
The gradient wind speed above is converted to surface wind speed using a factor of 0.8 (Vickery et al., 2009a; Vickery et al., 2000a). However, structural damage is more related to peak gust wind speed which is the maximum instantaneous wind speed. Therefore, a gust factor is needed to convert the surface wind speed. The gust model developed by Engineering Sciences Data Unit (ESDU, 1982, 1983) has been shown to be adequate for modeling gust factors (Xu \& Brown, 2008b). Xu and Brown (2008b) conducted a 1000year simulation to estimate the 3-sec gust factor using the ESDU model and found that the distribution of the calculated values of the factor is highly concentrated around 1.287 with a standard deviation of 0.002 . This value has been adopted for use in this research.

The study area, in this case, is relatively small compared to the size of the hurricane. Consequently, the variation of wind speed across the study area is very minimal. Therefore, only the maximum wind speed at the middle of the power distribution system is considered and all the poles are assumed to be subjected to this particular wind speed. Figure 4.10 shows the variation of the surface wind speed at the middle of the distribution system at various time intervals as the hurricane travels along its track.

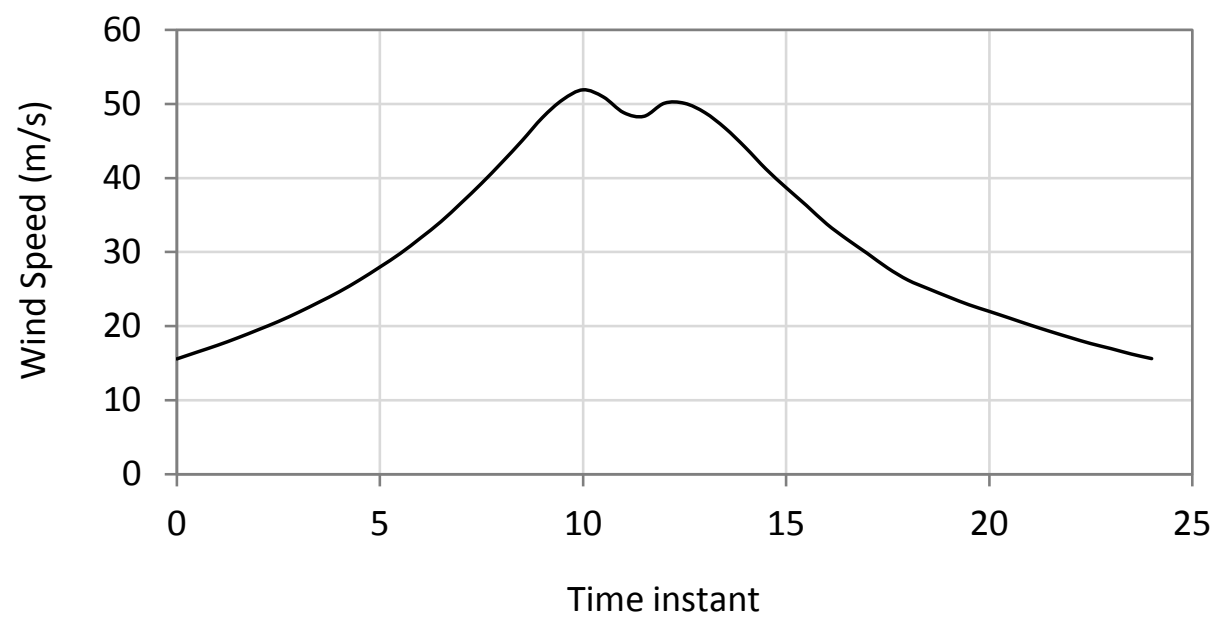

Figure 4.10 Surface wind speed variation at the center of study area 


\subsection{System Reliability Evaluation}

The system reliability model presented in Chapter 3 is used here. The reliability of line 33 (which has 19 poles) in Figure 4.3 over time is plotted in Figure 4.11 as an example using the lower and upper bounds of the probabilities of failure of the line from probabilistic hurricane analysis. As expected, the reliability decreases over time as the poles in the line decay. The lower and upper bounds of the probabilities of failure of line 33 for the scenariobased hurricane analysis are 0.53 and 1.00 respectively, calculated assuming the hurricane strikes when the poles are new. It should be noted that failure of cross arms is neglected in the above analysis. The probabilities of failure of end poles are also ignored since they are an order of magnitude smaller than the probabilities of failure of intermediate poles.

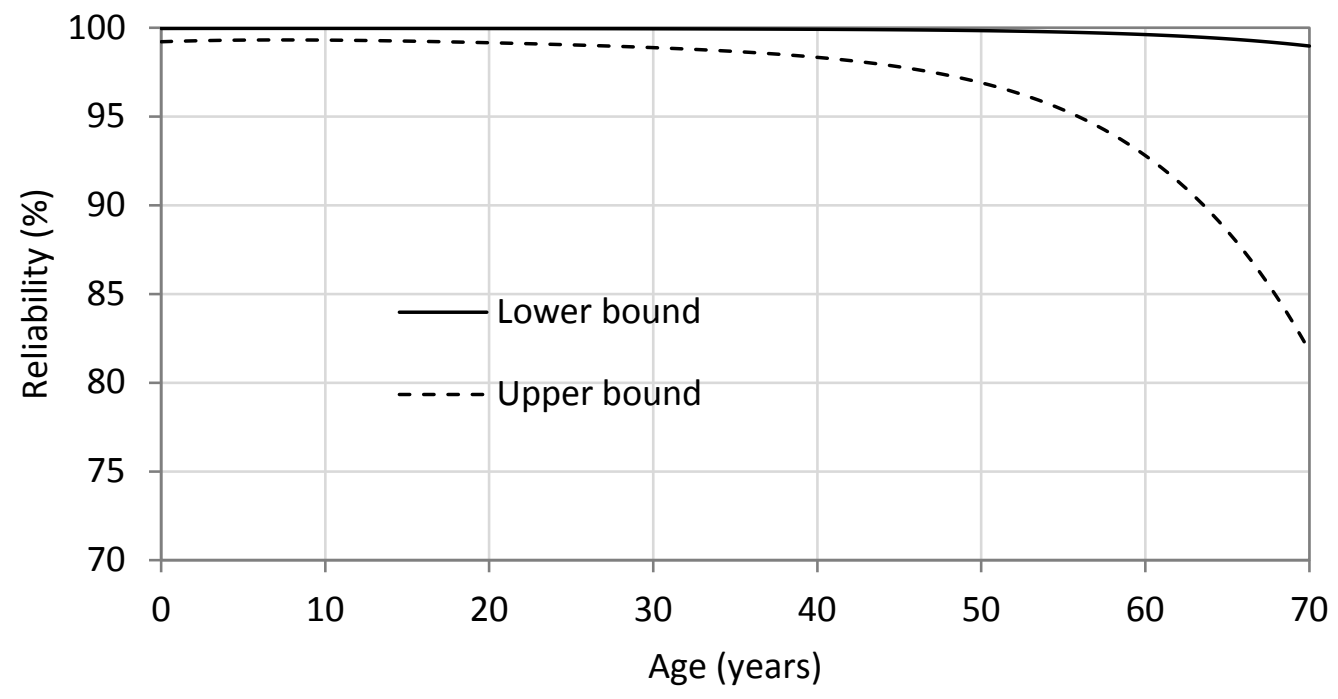

Figure 4.11 Reliability of line 33 with age using probabilistic hurricane analysis

As stated earlier, there are an estimated 434 residential, 15 industrial, and 9 commercial/institutional customers in the Micropolis including 3 schools and 3 churches. Industrial customers are served by line 9 while commercial centers are concentrated around 
line 44 as can be seen from Figure 4.2. Three-phase feeder lines branched off to singlephase laterals to deliver power to the customers through 'service drops' from distribution transformers. For simplicity sake, Bagchi et al. (2009) categorized the amount of load consumed by customers in Micropolis into few distinct profiles with all customers belonging to each profile having a fixed load demand (kVA) all through the year. However, in this research, new average demand has been assigned to each customer group based on the average power consumption of customers of a utility company in Florida obtained from EIA (2013). It is assumed that all customers have a fixed demand shown in Table 4.4 throughout the year. Based on the fixed consumption, the load served by each line is the sum of all loads downstream of the line.

Table 4.4 Load profiles for consumers across Micropolis

\begin{tabular}{ll}
\hline \hline Customer type & Consumption \\
\hline Residential & $1.5 \mathrm{~kW} / \mathrm{h}$ \\
City churches & $5 \mathrm{~kW} / \mathrm{h}$ \\
City schools & $10 \mathrm{~kW} / \mathrm{h}$ \\
Industrial (Feeder 1) & $39.4 \mathrm{~kW} / \mathrm{h}$ \\
Central business district (Feeder 2) & $10.1 \mathrm{~kW} / \mathrm{h}$ \\
\hline Feeder 1 total & $\mathbf{1 , 3 3 4 k W / h}$ \\
Feeder 2 total & $\mathbf{3 9 4 k W / h}$ \\
System total & $\mathbf{1 , 7 2 8 k W / h}$ \\
\hline \hline
\end{tabular}

The system reliability is plotted in Figure 4.12 using the annual probabilities of failure calculated using probabilistic hurricane analysis. The system reliability is calculated using both the upper and lower bounds of probabilities of failure of the lines evaluated previously. The system reliability using the upper bounds is $97.8 \%$ and $60.2 \%$ at 0 and 70 years respectively. Using the lower bounds, the reliability is $99.6 \%$ and $96.8 \%$ at 0 and 70 years respectively. The system reliability for the event-based analysis was calculated to be $15.3 \%$ and $3.2 \%$ for the lower and upper bounds of line failures respectively. 


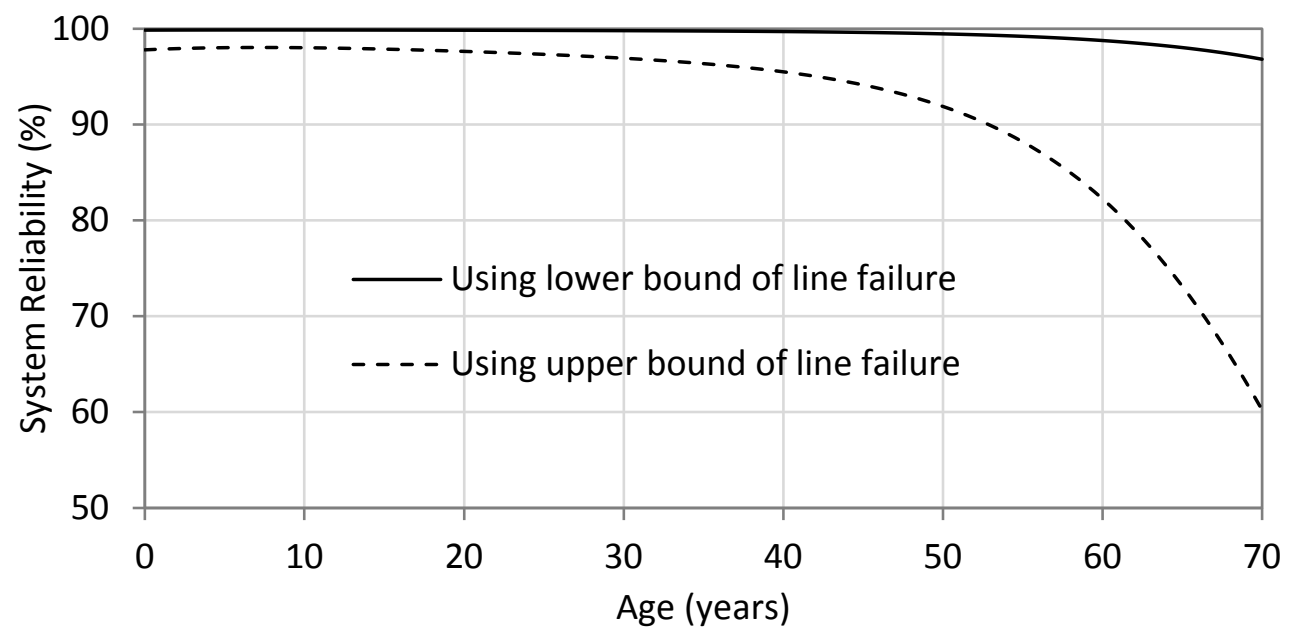

Figure 4.12 System reliability with age using probabilistic hurricane analysis

\subsection{Component Importance Index}

In a given system, some components are more important for the reliability of the system than others. Determining the relative importance of all components/lines in the distribution system is essential for targeted hardening. The component importance index is usually used in probabilistic risk assessment to identify components and subsystems whose reliability need to be improved to reduce risk to the whole system (Rausand \& Høyland, 2004). One of the methods of evaluating the relative importance of system components is risk achievement worth (RAW). RAW is a measure of the "worth" of component $i$ in achieving system reliability. It is defined as the ratio of the conditional system unreliability if component $i$ has failed to the actual system unreliability. RAW of component $i$ is defined as (Rausand \& Høyland, 2004):

$$
R A W(i)=\frac{1-R_{S}\left(Q_{i}=1\right)}{1-R_{S}} \quad \text { for } i=1,2, \ldots, n
$$


where $R_{S}\left(Q_{i}=1\right)$ is the system reliability when component (line) $i$ has failed while $R_{S}$ is the reliability of the original system. The risk achievement worth of all the lines in the system is calculated using the upper bounds of line failures when the poles are new and presented in Figure 4.13.

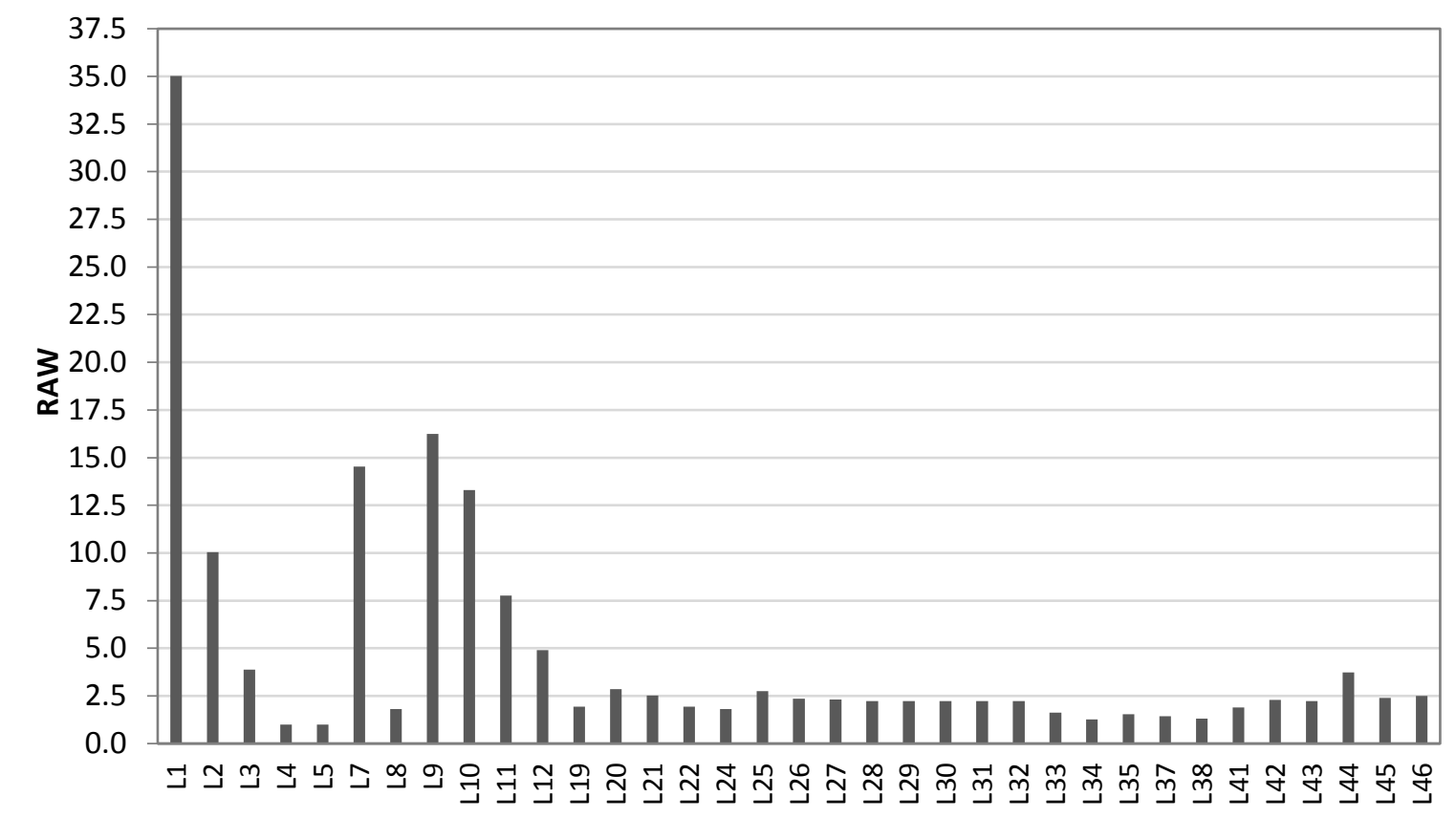

Figure 4.13 Risk achievement worth of lines

It can be seen from Figure 4.13 that as expected, lines with higher loads have higher risk achievement worth. The main feeder lines have higher RAW than the laterals because the delivery of power to the laterals depends on the reliability of the feeder lines. For example, line 1 has the highest RAW because the delivery of power to all the laterals on feeder 1 depends on the reliability of line 1. Line 9 on the other hand, have a relatively high RAW because it serves an industrial area with high power consumption. This will guide decisionmaking when selecting the part of the system to be strengthened. 


\subsection{Targeted Hardening Strategies}

Three strategies will be considered to demonstrate the proposed framework. These are:

i. Strategy 1: Hardening only the main feeder lines

ii. Strategy 2: Hardening all lines with RAW $\geq 2.5$

iii. Strategy 3: Hardening all lines

In all the above three cases, hardening means using a pole that is one class higher than that designed previously. This means using class 3 and 4 poles instead of class 4 and 5 for the three-phase main feeder lines and single-phase laterals, respectively. Both strategies 1 and 2 are considered as targeted hardening measures. Strategy 1 is chosen because power delivery to all the laterals lines depends on the reliability of the main feeder lines. However, some lateral lines serving heavy loads have higher RAW than some feeder lines as can be seen from Figure 4.13. Hence, strategy 2 is chosen and RAW set at $\geq 2.5$ so as to harden both feeder and lateral lines that have a significant effect on system reliability. Strategy 3 is chosen to investigate the difference between targeted hardening and hardening the whole system.

Figure 4.14 shows the improvement in system reliability using all three strategies for probabilistic hurricane analysis. The system reliability is evaluated using the upper bound of line failure probability. System reliability at 0 years is improved from $97.8 \%$ to $99.1 \%$, $99.2 \%$, and $99.5 \%$ using strategies 1,2 , and 3 respectively. At 70 years, i.e. with poles that are 70 years old, the system reliability is improved from $60.2 \%$ to $73.4 \%, 75.2 \%$, and $79.6 \%$ using strategies 1,2 , and 3 respectively. 


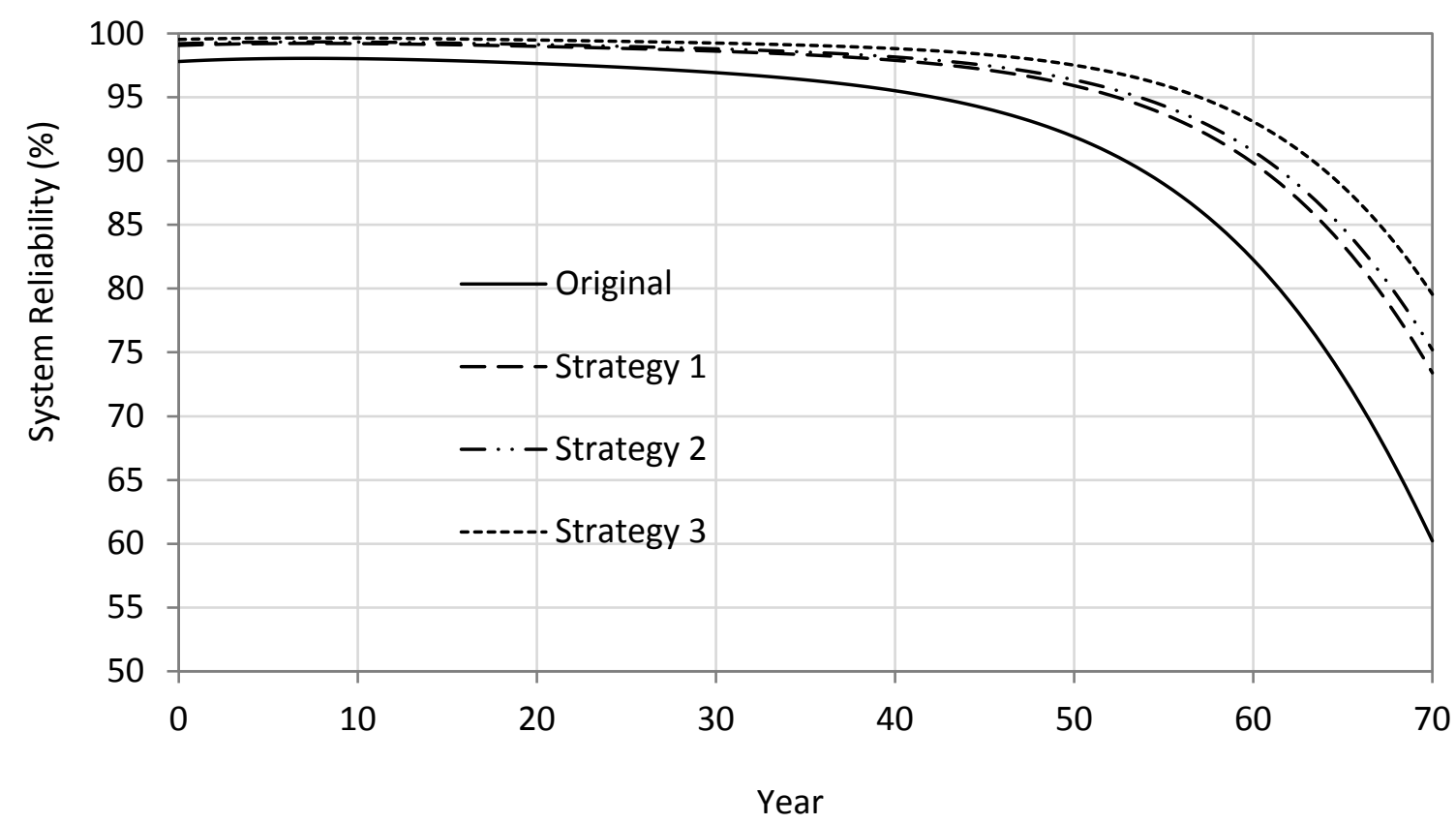

Figure 4.14 System reliability improvement after targeted hardening

For the scenario analysis, the system reliability improvements are shown in Table 4.5. There is no specific requirement on the level of reliability required for a power distribution system. Each utility company can assign a required level of reliability based on which the level of hardening can be selected.

Table 4.5 System reliability comparison for scenario-based analysis

\begin{tabular}{l|l|l}
\hline \hline State of System & Reliability using lower bounds & Reliability using upper bound \\
\hline Original system & $15.3 \%$ & $3.2 \%$ \\
Strategy 1 & $36.8 \%$ & $3.2 \%$ \\
Strategy 2 & $40.3 \%$ & $3.2 \%$ \\
Strategy 3 & $47.3 \%$ & $3.2 \%$ \\
\hline \hline
\end{tabular}




\subsection{Cost Analysis}

Utility companies and governments are concerned about the monetary costs and benefits of any hardening or disaster mitigation strategy. Decisions are usually made by considering the cost effectiveness of hardening methods. To evaluate the cost-effectiveness of the three proposed strategies above, cost analysis is performed for both probabilistic and scenariobased hurricane analysis. The net benefit, defined as benefit minus cost, is evaluated for each hardening strategy. The benefit of a hardening strategy is the reduction in damages associated with the hardening strategy while the cost is the cost of implementing the hardening strategy.

\subsubsection{Probabilistic Hurricane Analysis}

Life cycle cost analysis is performed for the probabilistic hurricane analysis. The method proposed by Chang (2003) has been adopted for the cost analysis as it considers indirect costs. The various costs considered are explained below.

i. Mitigation $\operatorname{cost}\left(\mathrm{C}_{\mathrm{M}}\right)$ : this is the cost of hardening the system using the 3 proposed strategies. It includes the cost of buying new stronger poles and installation cost.

ii. Maintenance cost: this is the cost of periodic maintenance carried out by utility companies. This includes cost of preventive maintenance, tree trimming, wildlife protection, and so on. The maintenance cost for the entire life span of a system is given by:

$$
C_{m}=\sum_{t} \sum_{i} m_{i}\left(x_{i}, t\right) \cdot(1+y)^{-t}
$$


where $m_{i}$ is the annual maintenance cost for element $i$ which depends on material properties $x_{i}$ and the year of analysis $t ; z(t)$ is a discount factor; and $y$ is the discount rate.

iii. Repair cost: this is the cost of repairing the damage to the system due to hurricanes. In this case, it is the cost of replacing failed poles. Failed poles are replaced with new poles of the same class. Consequently, the age distribution of poles in the system changes every year. The repair cost for the entire lifespan of the system is given by:

$$
C_{r}=\sum_{t} \sum_{i}\left[F_{i}\left(x_{i}, t\right) \cdot r_{i}\right] \cdot z(t)
$$

where $F_{i}$ is the annual probability of failure of element $i$, and $r_{i}$ is the unit repair cost of element $i$.

iv. Revenue loss: this is the cost incurred by the utility company due to interruptions in power supply to the customers caused by damage due to hurricanes. The total loss in revenue is given by:

$$
C_{v}=\sum_{t} V \cdot p \cdot z(t)
$$

where $V$ is the annual demand not met; and $p$ is the unit price of electricity. $V$ is a function of time to completely restore the system which in turn depends on the amount of damage (number of failed poles) and the number of repair crew units. Ouyang and Dueñas-Osorio (2014) assumed the restoration time for each failed pole to follow a normal distribution with a mean of 5 hours and standard deviation of 2.5 hours. Brown (2009) estimated the mean time to restoration for overhead line failure to be 4 hours. Here, it is assumed that the mean time to restore a failed line due to pole failure is 4 hours. It is also assumed that on average, there will be 
1 repair crew unit allocated to the city throughout a year. It is acknowledged that the number of repair crews varies between disaster and non-disaster periods. This will be better reflected in the scenario-based hurricane analysis.

The average annual outage hours for each line is found by multiplying the number of failed poles by the time to restore each pole. The number of failed poles is the product of the annual probability of failure for each pole group and the total number of poles for that age group. Finally, the annual unmet demand for each line is the product of average annual outage hours and total hourly consumption in the line.

v. Societal economic loss: this is the direct economic loss resulting from the interruption in power supply. The total economic loss over the lifespan of the system is given by:

$$
C_{e}=\sum_{t} E \cdot z(t)
$$

where $E$ is the expected annual direct economic loss. $E$ for each line is the product of average annual outage hours and monetary loss per hour. The monetary loss per hour for each customer category is adopted from LaCommare and Eto (2006) and shown in Table 6.

The total life cycle cost is then calculated using Equation (4.20).

$$
L C C=C_{M}+C_{m}+C_{r}+C_{v}+C_{e}
$$

To perform the life-cycle cost analysis, the lifespan of the system is required. In this case, the lifespan of the system is assumed to be the same as the life span of the distribution poles. The service life of wood poles has been a subject of much discussion. According to the NESC (2002), wood poles should be replaced or reinforced when their strength falls 
below $67 \%$ of the initial strength. Most utilities use 30 to 40 years as an estimated service life (Mankowski et al., 2002). However, research and several surveys show that the service life of wood poles can range from 60 to 80 years depending on specie, location, and maintenance (Morrell, 2008; Stewart, 1996). Datla and Pandey (2006) determined the approximate life expectancy of wood poles to be 69 years based on a study of 100,000 distribution poles.

The time scale considered for the life-cycle cost analysis is 70 years based on the amount of time it takes for the poles to degrade to approximately $67 \%$ of their original strength as plotted in Figure 4.5. In carrying out the life-cycle cost analysis, the following assumptions are made:

i. Maintenance cost remains constant and is the same for original and hardened system. Hardening the system might reduce the annual maintenance cost but there is a lack of data to quantify this potential reduction.

ii. Demand remains constant over the years

iii. Cost per unit of electricity remains constant

All the information needed for the life-cycle cost analysis is given in Table 4.6. 
Table 4.6 Life cycle cost analysis parameters

\begin{tabular}{lll}
\hline \hline Parameters & Value & Source \\
\hline Class 3 SP poles $(13.7 \mathrm{~m})$ & $\$ 544 /$ pole & ATS (2014) \\
Class 4 SP poles $(13.7 \mathrm{~m})$ & $\$ 479 /$ pole & ATS (2014) \\
Class 5 SP poles $(13.7 \mathrm{~m})$ & $\$ 441 / \mathrm{pole}$ & ATS (2014) \\
Cost of pole replacement under normal condition & $\$ 2,500 /$ pole & Taras et al. (2004) \\
Cost of pole replacement under storm condition & $\$ 4,000 /$ pole & Xu and Brown (2008b) \\
Annual maintenance cost, $m_{i}$ & $\$ 4,000 /$ circuit mile & Francis et al. (2011) \\
Discount rate, $y$ & & Bastidas-Arteaga \\
& $4 \%$ & Stewart (2015) \\
Unit price, $p$ & $\$ 0.11 / \mathrm{kWh}$ & Xu and Brown (2008b) \\
Economic loss (residential) & $\$ 2.70 / \mathrm{h}$ & LaCommare and Eto (2006) \\
Economic loss (commercial) & $\$ 886 / \mathrm{h}$ & LaCommare and Eto (2006) \\
Economic loss (industrial) & $\$ 3,253 / \mathrm{h}$ & LaCommare and Eto (2006) \\
Average consumption (residential) & $1.5 \mathrm{~kW} / \mathrm{h}$ & EIA (2013) \\
Average consumption (commercial) & $10.1 \mathrm{~kW} / \mathrm{h}$ & EIA (2013) \\
Average consumption (industrial) & $39.4 \mathrm{~kW} / \mathrm{h}$ & EIA (2013) \\
Average consumption (schools) & $10 \mathrm{~kW} / \mathrm{h}$ & Assumed \\
Average consumption (churches) & $5 \mathrm{~kW} / \mathrm{h}$ & Assumed \\
\hline \hline
\end{tabular}

Two cases are considered for the life-cycle cost analysis. Case 1 is when the hardening strategies are carried out at the construction stage of a new system, i.e. the utility company decided to build a stronger system from the beginning. In this case, the mitigation cost is the additional cost needed to use stronger poles instead of the poles that have been shown to be sufficient during the design.

In Case 2, it is assumed the system exist as it is and proactive hardening strategies are employed. In this case, however, it will illogical to assume the hardening measures will be carried out few years after the distribution system is constructed. According to Bjarnadottir et al. (2014), the age of distribution poles in Florida ranges from 0 to 50 years. For Case 2, it is assumed that the system and all the poles are 20 years old when the hardening measures are carried out. In this case, the mitigation cost includes the cost of buying new stronger 
poles and the cost of removing and replacing existing poles. The life cycle cost analysis is then performed considering a life span of 50 years which is how much longer the unhardened system is expected to last.

\subsubsection{Case 1: hardening at construction stage}

The results of the LCC analysis for Case 1 of the probabilistic hurricane analysis are shown in Table 4.7. It can be seen from the table that as expected, strengthening measures lowers the repair cost, revenue loss, and economic loss. It can be seen that all three strengthening strategies result in lower life-cycle cost compared to the un-hardened system with strategy 3 providing the highest net benefit. The savings as a result of hardening comes mostly from a reduction in repair cost and societal economic losses which more than made up for the initial hardening investment. Strategy 3, which entails hardening the entire system, has the lowest life cycle cost because the difference in the costs of the three classes of poles is small. For example, a class 3 pole cost about $\$ 65$ more than a class 4 pole. The effect of this can be seen in Table 4.7 where it costs an additional $\$ 29,000$ to use stronger poles to construct the whole system from the beginning.

Table 4.7 also demonstrates the importance of various components of the life cycle cost. For the unhardened system, about $48 \%$ of total cost are economic losses suffered by the customers due to power outages. This dropped to an average of $31 \%$ with the 3 hardening strategies. It can also be noted that routine maintenance costs outweigh repair costs and revenue losses combined. For example, for the unhardened system, the maintenance cost constitutes about $39 \%$ of the total cost compared to repair cost and revenue loss which combined constitutes about $13 \%$ of the total cost.

From a utility company's perspective, the total cost excluding the societal economic losses may be of more interest. All three hardening measures have lower life cycle cost than the unhardened system even if economic losses are excluded with strategy 3 having the lowest 
cost. Again, this is due to the low additional cost needed to construct a system with stronger poles.

Table 4.7 LCCA results for probabilistic hurricane analysis - Case $1(\$ 1,000 \mathrm{~s})$

\begin{tabular}{lllll}
\hline \hline Cost category & $\begin{array}{l}\text { Unhardened } \\
\text { system }\end{array}$ & Strategy 1 & Strategy 2 & Strategy 3 \\
\hline Mitigation cost, $C_{M}$ & - & 9 & 14 & 29 \\
Maintenance cost, $C_{m}$ & 1,762 & 1,762 & 1,762 & 1,762 \\
Repair cost, $C_{r}$ & 588 & 514 & 480 & 268 \\
Revenue loss, $C_{v}$ & 8 & 5 & 4 & 4 \\
Societal Economic loss, $C_{e}$ & 2,155 & 1,050 & 992 & 978 \\
\hline Total LCC & $\mathbf{4 , 5 1 3}$ & $\mathbf{3 , 3 3 9}$ & $\mathbf{3 , 2 5 3}$ & $\mathbf{3 , 0 4 1}$ \\
Total LCC w/o $C_{\boldsymbol{e}}$ & $\mathbf{2 , 3 5 8}$ & $\mathbf{2 , 2 9 0}$ & $\mathbf{2 , 2 6 0}$ & $\mathbf{2 , 0 6 2}$ \\
\hline Net benefit & - & $\mathbf{1 , 1 7 4}$ & $\mathbf{1 , 2 6 0}$ & $\mathbf{1 , 4 7 2}$ \\
Net benefit w/o $C_{\boldsymbol{e}}$ & - & $\mathbf{6 8}$ & $\mathbf{9 8}$ & $\mathbf{2 9 6}$ \\
\hline \hline
\end{tabular}

During disasters such as hurricanes, considerable damage can occur to buildings and other infrastructure systems other than power systems. This usually reduces economic activities even if the power system is not damaged. It also reduces electricity consumption as businesses close and residents evacuate. To account for this reduction, the revenue and economic losses in Table 4.7 are reduced. However, due to lack of literature on the estimate of the reduction in economic activities and power consumption, four levels of reduction, $10 \%, 20 \%, 30 \%$, and $50 \%$, are considered as shown in Table 4.8. It can be seen from Table 4.8 that the pattern of the total life cycle cost after reductions of economic and revenue losses remains the same as that in Table 4.7. All three strategies resulted in positive net benefit with strategy 3 having the highest. However, the contribution of the economic loss to the total life cycle cost reduces as expected. 
Table 4.8 LCCA results with reductions in revenue and economic losses $(\$ 1,000 \mathrm{~s})$

\begin{tabular}{llll}
\hline \hline Cost & Strategy 1 & Strategy 2 & Strategy 3 \\
\hline Net benefit with 10\% reduction in $C_{v}$ and $C_{e}$ & 1,063 & 1,144 & 1,354 \\
Net benefit with 20\% reduction in $C_{v}$ and $C_{e}$ & 952 & 1,027 & 1,236 \\
Net benefit with 30\% reduction in $C_{v}$ and $C_{e}$ & 841 & 910 & 1,118 \\
Net benefit with 50\% reduction in $C_{v}$ and $C_{e}$ & 620 & 677 & 882 \\
\hline \hline
\end{tabular}

\subsubsection{Case 2: hardening 20 year-old system}

The results of the LCC analysis for Case 2 are shown in Table 4.9. Considering the total life cycle cost, all three hardening measures have lower total costs than the unhardened system. Among the 3 strategies, strategy 1 gives the highest net benefit followed by strategy 2. Strategy 3 gives the lowest net benefit among the three. This shows the importance of targeted hardening measures for existing systems because it implies that unlike in Case 1, it is more economical to employ targeted hardening than hardening the whole system. If societal economic losses are excluded, it can be seen that none of the hardening strategies is cost effective as implied by the negative net benefit. This is due to the high cost of carrying out the mitigation strategies because existing poles need to be removed, and stronger poles need to be purchased and installed. 
Table 4.9 LCCA results for probabilistic hurricane analysis - Case $2(\$ 1,000 \mathrm{~s})$

\begin{tabular}{lllll}
\hline \hline Cost category & $\begin{array}{l}\text { Unhardened } \\
\text { system }\end{array}$ & Strategy 1 & Strategy 2 & Strategy 3 \\
\hline Mitigation cost, $C_{M}$ & - & & & \\
Maintenance cost, $C_{m}$ & 1,614 & 424 & 652 & 1,978 \\
Repair cost, $C_{r}$ & 689 & 1,614 & 1,614 & 1,614 \\
Revenue loss, $C_{v}$ & 9 & 580 & 526 & 199 \\
Societal Economic loss, $C_{e}$ & 2,524 & 5 & 4 & 3 \\
\hline Total LCC & $\mathbf{4 , 8 3 6}$ & 821 & 729 & 707 \\
Total LCC w/o $C_{\boldsymbol{e}}$ & $\mathbf{2 , 3 1 2}$ & $\mathbf{3 , 4 4 3}$ & $\mathbf{3 , 5 2 5}$ & $\mathbf{4 , 5 0 1}$ \\
\hline Net benefit & $\mathbf{-}$ & $\mathbf{2 , 6 2 2}$ & $\mathbf{2 , 7 9 5}$ & $\mathbf{3 , 7 9 4}$ \\
Net benefit w/o $C_{\boldsymbol{e}}$ & - & $\mathbf{1 , 3 9 2}$ & $\mathbf{1 , 3 1 1}$ & $\mathbf{3 3 5}$ \\
\hline \hline
\end{tabular}

When a reduction in overall economic activities and electricity demand due to damage to buildings and other infrastructure systems is taken into account, the total life cycle costs with four levels of reductions are shown in Table 4.10. It can be seen that with a reduction of $10 \%$ in both economic and revenue losses, the pattern is the same as in Table 9 with all three strategies having positive net benefits. However, with a reduction of $20 \%$ and higher, only strategies 1 and 2 are cost effective.

Table 4.10 LCCA results with reductions in revenue and economic losses $(\$ 1,000 \mathrm{~s})$

\begin{tabular}{l|l|l|l}
\hline \hline Cost & Strategy 1 & Strategy 2 & Strategy 3 \\
\hline Net benefit with $10 \%$ reduction in $C_{v}$ and $C_{e}$ & 1,221 & 1,131 & 152 \\
Net benefit with $20 \%$ reduction in $C_{v}$ and $C_{e}$ & 1,051 & 951 & -30 \\
Net benefit with 30\% reduction in $C_{v}$ and $C_{e}$ & 880 & 771 & -212 \\
Net benefit with 50\% reduction in $C_{v}$ and $C_{e}$ & 539 & 411 & -577 \\
\hline \hline
\end{tabular}




\subsubsection{Scenario-based analysis}

For the scenario-based hurricane analysis, the total incurred costs due to hurricane damage as well as costs of mitigation are computed. The incurred costs due to hurricane damage include repair cost, revenue loss, and economic losses. The repair cost is calculated using the number of poles that fail which is found by multiplying the probability of failure by the number of poles. The revenue and economic losses are calculated by assuming that the average time to repair a failed pole is 4 hours. For a category 3 hurricane such as the one considered here, utility companies drastically increases the number of repair crew units for affected areas including hiring external crews from other utility companies (Xu \& Brown, $2008 \mathrm{~b}$ ). Here, it is assumed that $6 \mathrm{crew}$ units are assigned to the city for speedy restoration.

As in the case of probabilistic hurricane analysis, two cases have been considered here as well. Case 1 is when the system is constructed initially with stronger poles based on the 3 hardening measures and the hurricane strikes the city in the year the system was constructed. Case 2 is hardening an existing 20-year old system and the hurricane strikes the city immediately following the hardening measures.

\subsubsection{Case 1: hardening at construction stage}

The results of the cost analysis for Case 1 are shown in Table 4.11. It can be seen that the pattern is similar to the results for the probabilistic hurricane analysis. All the three hardening strategies results in lower incurred cost compared with the unhardened system with strategy 3 providing the highest benefit followed by strategy 2 . However, in this case, repair costs are considerably higher than economic losses. The repair costs constitute $64 \%$, $75 \%, 75 \%$, and $60 \%$ for the unhardened system, and strategy 1,2 , and 3 , respectively. 
Table 4.11 Cost analysis results for scenario hurricane - Case 1 (\$1,000s)

\begin{tabular}{lllll}
\hline \hline Cost category & $\begin{array}{l}\text { Unhardened } \\
\text { system }\end{array}$ & Strategy 1 & Strategy 2 & Strategy 3 \\
\hline Mitigation cost, $C_{M}$ & - & & & \\
Repair cost, $C_{r}$ & 1,594 & 1,400 & 1,291 & 663 \\
Revenue loss, $C_{v}$ & 3 & 2 & 2 & 1 \\
Societal Economic loss, $C_{e}$ & 906 & 448 & 421 & 414 \\
\hline Total Cost & $\mathbf{2 , 5 0 3}$ & $\mathbf{1 , 8 5 9}$ & $\mathbf{1 , 7 2 7}$ & $\mathbf{1 , 1 0 8}$ \\
Total Cost w/o $C_{\boldsymbol{e}}$ & $\mathbf{1 , 5 9 7}$ & $\mathbf{1 , 4 1 1}$ & $\mathbf{1 , 3 0 7}$ & $\mathbf{6 9 3}$ \\
\hline Net benefit & - & $\mathbf{6 4 5}$ & $\mathbf{7 7 6}$ & $\mathbf{1 , 3 9 6}$ \\
Net benefit w/o $C_{\boldsymbol{e}}$ & $\mathbf{-}$ & $\mathbf{1 8 6}$ & $\mathbf{2 9 0}$ & $\mathbf{9 0 4}$ \\
\hline \hline
\end{tabular}

Table 4.12 shows the results of the total life cycle costs with reductions in economic and revenue losses. It can be seen that the pattern is the same as in Table 4.11 where all the 3 hardening strategies result in savings compared with the unhardened system with strategy 3 giving the lowest cost saving.

Table 4.12 Cost analysis results with reductions in revenue and economic losses $(\$ 1,000 \mathrm{~s})$

\begin{tabular}{llll}
\hline \hline Cost & Strategy 1 & Strategy 2 & Strategy 3 \\
\hline Net benefit with 10\% reduction in $C_{v}$ and $C_{e}$ & 599 & 727 & 1,346 \\
Net benefit with 20\% reduction in $C_{v}$ and $C_{e}$ & 553 & 679 & 1,297 \\
Net benefit with 30\% reduction in $C_{v}$ and $C_{e}$ & 507 & 630 & 1,248 \\
Net benefit with 50\% reduction in $C_{v}$ and $C_{e}$ & 415 & 532 & 1,149 \\
\hline
\end{tabular}

The implication of the uncertainties in the assumed values of repair time and number of repair crew units on net benefit of mitigation strategies has also been investigated. Figure 4.15 shows a plot of the net benefit when repair time is varied while the number of repair crew units is kept constant at 6 as initially assumed. It can be seen that the net benefit 
increases significantly as the repair time increases for all mitigation strategies. This implies that mitigation strategies are more cost-effective in cases where it takes utility companies longer to repair failed poles. This is important for decision making especially for areas where access is usually hindered after hurricanes. In such cases, adopting measures to reduce the level of damage can be highly cost-effective.

Figure 4.16 shows a plot of the net benefit with varying number of repair crew units while repair time is kept constant at 4 hours as initially assumed. It can be seen that the net benefit decreases as the number of repair crew units increases. However, the impact becomes insignificant as the number of crew units exceeds about 15 . This implies that for utility companies with few repair crew units or for distribution systems with few allocated repair crew units, deploying mitigation strategies can result in higher monetary benefit.

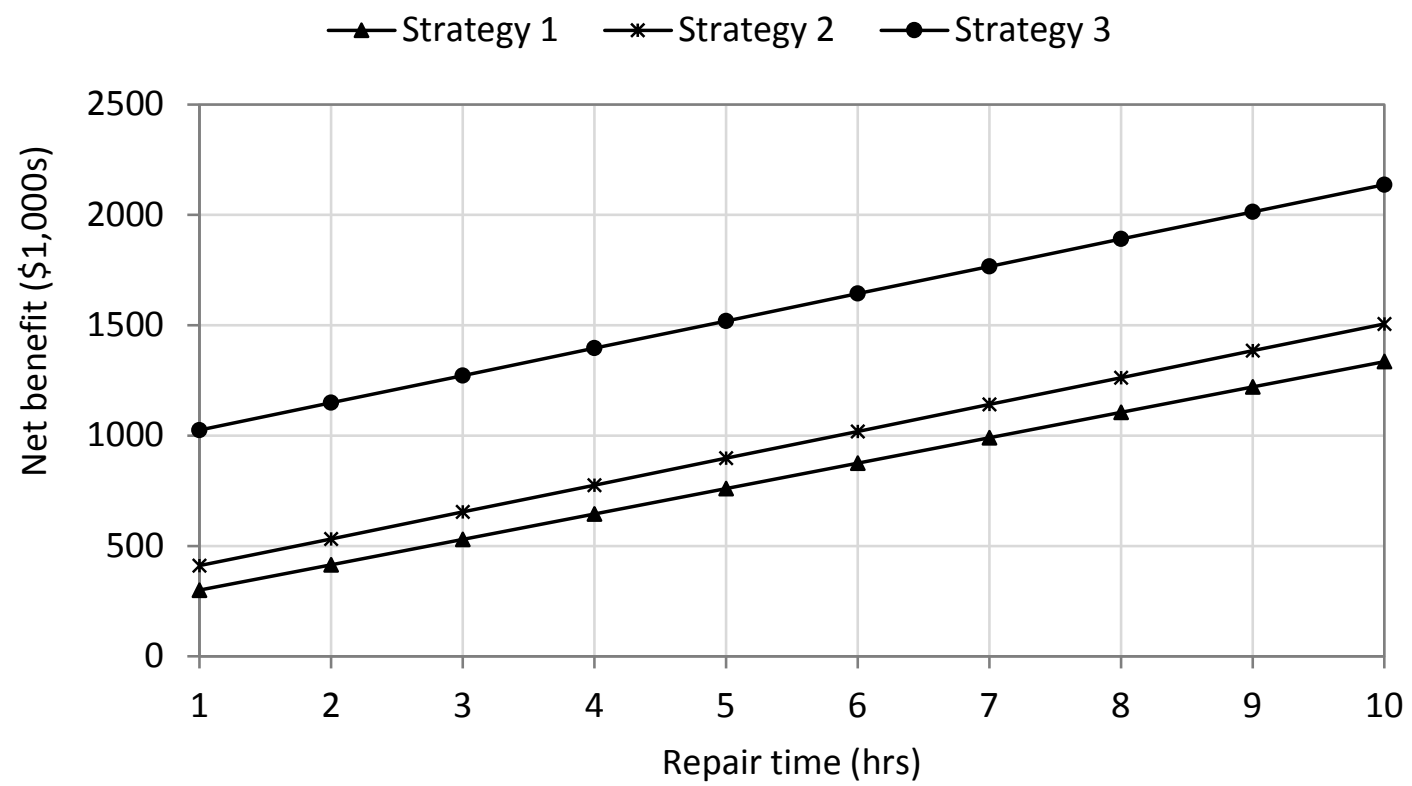

Figure 4.15 Impact of repair time on net benefit of mitigation strategies (Case 1) 


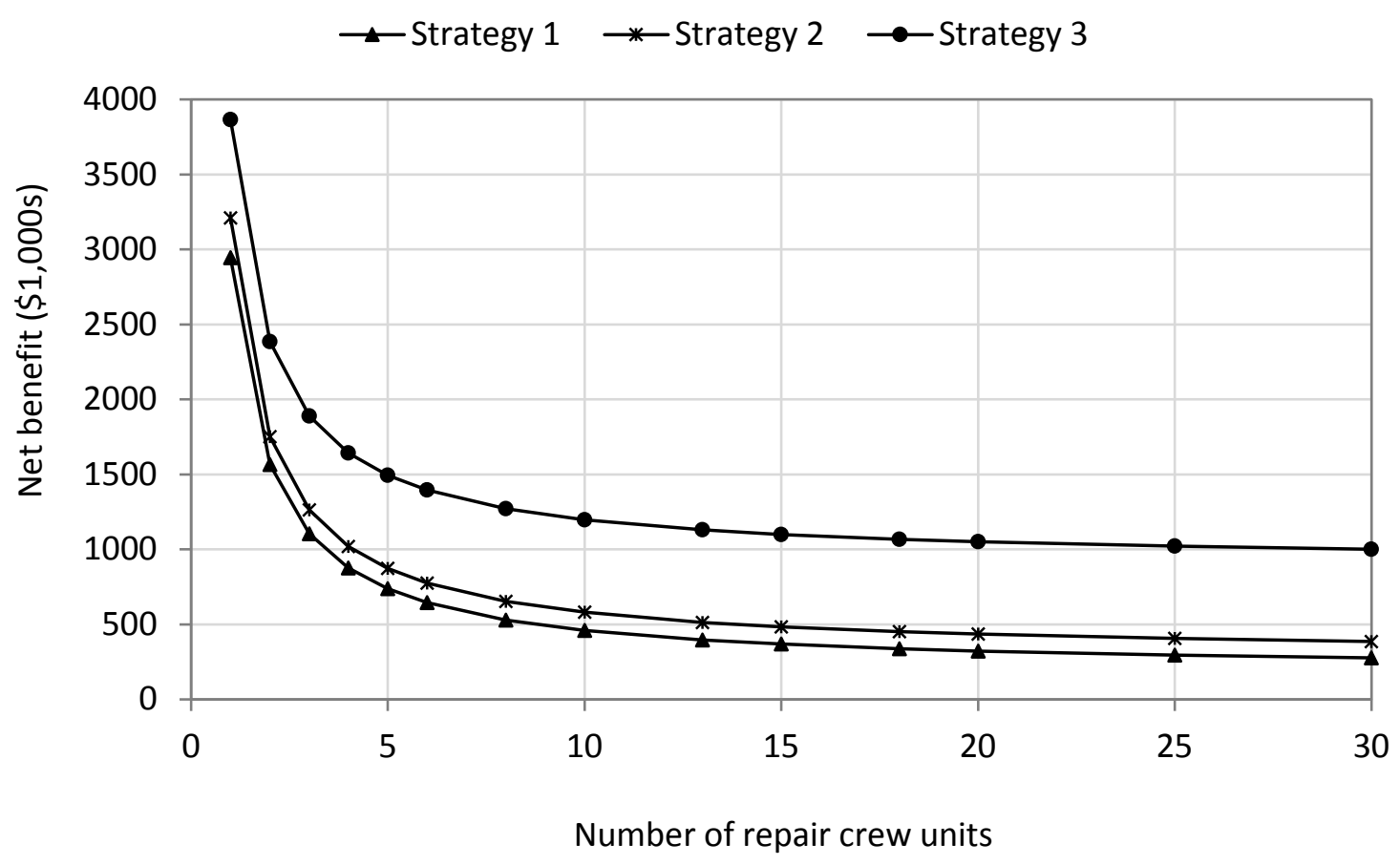

Figure 4.16 Impact of number of repair crew units on net benefit (Case 1)

\subsubsection{Case 2: hardening 20 year-old system}

The results for Case 2 are shown in Table 4.13. It can be seen that if total costs are considered, only strategies 1 and 2 are cost effective. Strategy 3 results in negative net benefit due to the high cost of mitigation. If societal economic losses are excluded, none of the hardening measures are cost effective. 
Table 4.13 Cost analysis results for scenario hurricane - Case $2(\$ 1,000 \mathrm{~s})$

\begin{tabular}{lllll}
\hline \hline Cost category & Unhardened system & Strategy 1 & Strategy 2 & Strategy 3 \\
\hline Mitigation cost, $C_{M}$ & - & 424 & 652 & 1,978 \\
Repair cost, $C_{r}$ & 1,625 & 1,425 & 1,312 & 663 \\
Revenue loss, $C_{v}$ & 3 & 2 & 2 & 1 \\
Societal Economic loss, & 922 & 449 & 421 & 414 \\
$C_{e}$ & & & & \\
\hline Total Cost & $\mathbf{2 , 5 5 1}$ & $\mathbf{2 , 3 0 0}$ & $\mathbf{2 , 3 8 7}$ & $\mathbf{3 , 0 5 7}$ \\
Total Cost w/o $C_{\boldsymbol{e}}$ & $\mathbf{1 , 6 2 9}$ & $\mathbf{1 , 8 5 1}$ & $\mathbf{1 , 9 6 6}$ & $\mathbf{2 , 6 4 3}$ \\
\hline Net benefit & - & $\mathbf{2 5 1}$ & $\mathbf{1 6 4}$ & $\mathbf{- 5 0 6}$ \\
Net benefit w/o $C_{\boldsymbol{e}}$ & $\mathbf{-}$ & $\mathbf{- 2 2 3}$ & $\mathbf{- 3 3 7}$ & $\mathbf{- 1 , 0 1 4}$ \\
\hline \hline
\end{tabular}

Table 4.14 shows the total life cycle costs after reductions in economic and revenue losses. For a reduction of $10 \%, 20 \%$, and $30 \%$, strategies 1 and 2 are cost-effective as before. However, for a reduction of $50 \%$, only strategy 1 is cost-effective. Strategy 3 is not costeffective for all reductions.

Table 4.14 Cost analysis results with reductions in revenue and economic losses $(\$ 1,000 \mathrm{~s})$

\begin{tabular}{lllc}
\hline \hline Cost & Strategy 1 & Strategy 2 & Strategy 3 \\
\hline Net benefit with 10\% reduction in $C_{v}$ and $C_{e}$ & 203 & 113 & -557 \\
Net benefit with 20\% reduction in $C_{v}$ and $C_{e}$ & 156 & 63 & -608 \\
Net benefit with 30\% reduction in $C_{v}$ and $C_{e}$ & 108 & 13 & -659 \\
Net benefit with 50\% reduction in $C_{v}$ and $C_{e}$ & 13 & -88 & -761 \\
\hline \hline
\end{tabular}

The impacts of repair time and number of repair crew units on net benefit of mitigation strategies are shown in Figure 4.17 \& Figure 4.18, respectively. From Figure 4.17, it can be seen that the cost-effectiveness of the mitigation strategies depends on the repair time. 
For example, strategy 3 is only cost effective when the average repair time is $\geq 8$ hours. From Figure 4.18, it can be seen that as the number of repair crew units increases, the mitigation strategies become ineffective. This is because increasing repair crew units decrease power outage hours which in turn decreases societal economic losses and revenue losses.

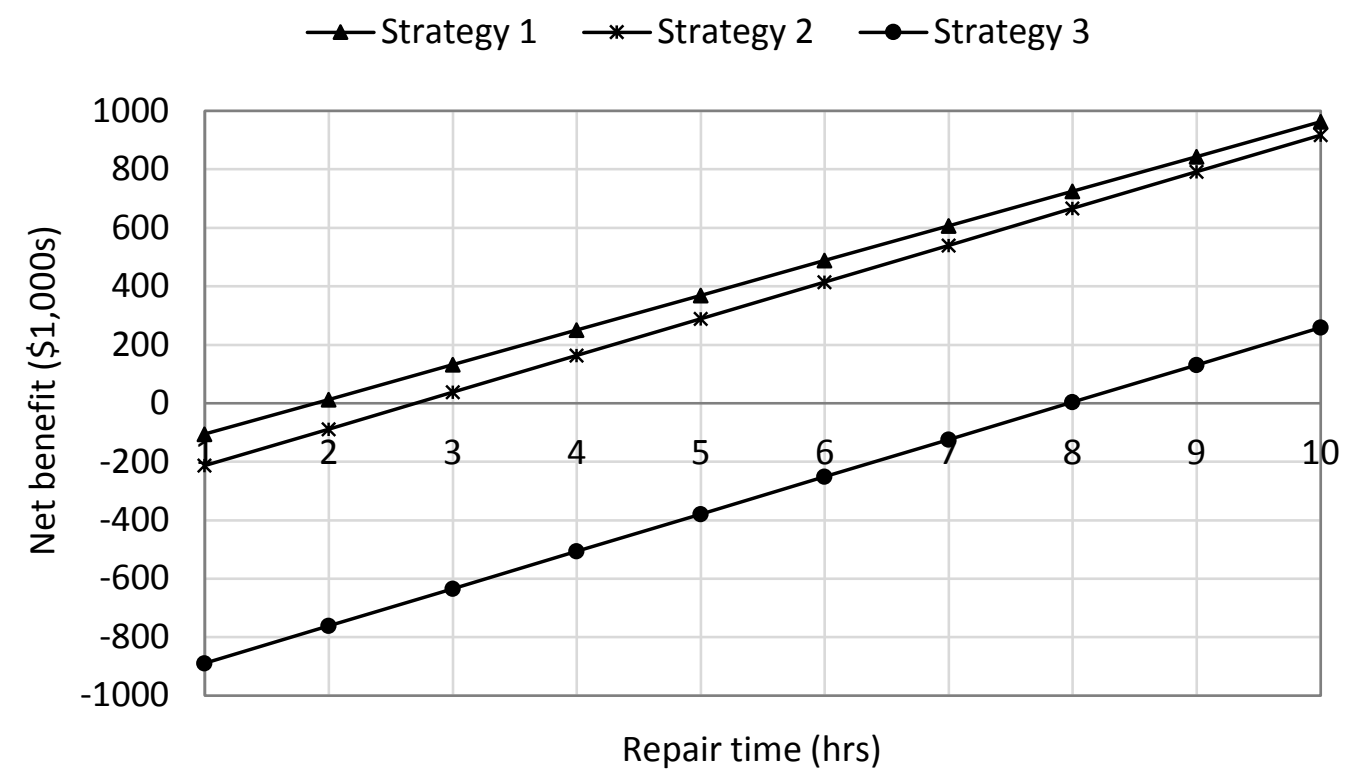

Figure 4.17 Impact of repair time on net benefit of mitigation strategies (Case 2) 


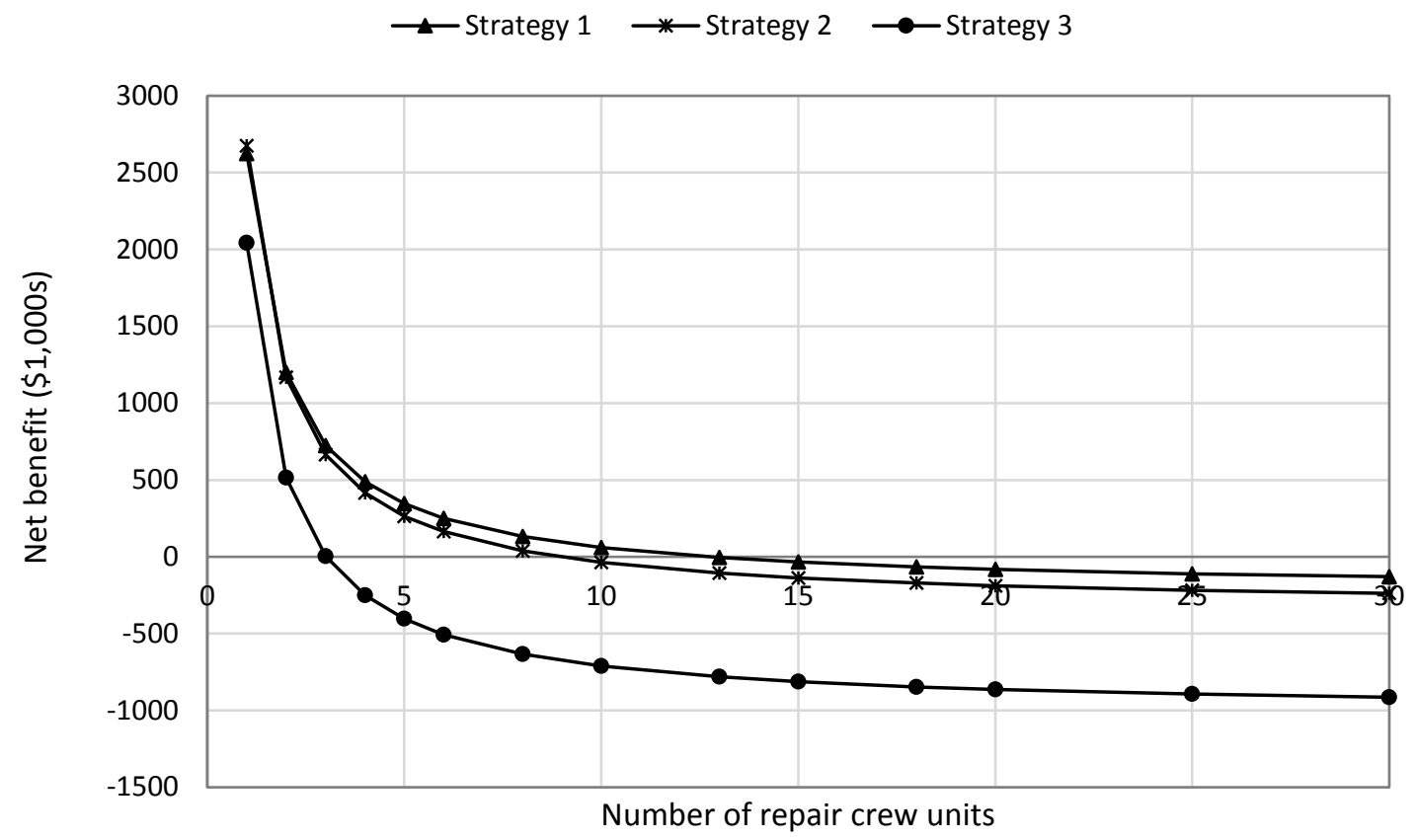

Figure 4.18 Impact of number of repair crew units on net benefit (Case 2)

\subsection{Conclusions}

This chapter presented a framework for evaluating the effectiveness of targeted hardening measures for power distribution systems subjected to hurricanes. The framework incorporated hurricane hazard analysis, system reliability evaluation, component importance measure, cost analysis as well as aging of support structures. A notional power distribution system was used to demonstrate the framework.

The results from the case study considered showed the importance of evaluating system reliability, component importance as well as targeted hardening of distribution systems. It was shown that hardening components or lines that have a greater impact on system reliability can be cost-effective in some cases. 
From the results, it can be concluded that strengthening an entire distribution system is only cost-effective for new systems. Using stronger poles than required by design for the entire system at construction stage of new systems resulted in lower life-cycle cost in all cases. However, for older systems, targeted hardening was shown to be cost-effective relative to hardening an entire system. The case study also showed that mitigation strategies can be cost-effective for distribution systems in less accessible areas or areas where fewer repair crew units are available which usually leads to prolonged repair time.

The results from the considered case study also showed the importance of considering economic losses in evaluating the cost effectiveness of hardening measures. It was shown that some hardening strategies that might not be cost effective for a utility company can be very cost effective if societal economic losses are considered. This is important especially for municipal utilities which are owned by city governments. In such cases, the high cost of mitigation measures or additional cost of constructing a stronger system can be easily compensated by the huge savings in economic losses. 


\section{Potential Impact of Climate Change on Distribution Systems Subjected to Hurricanes ${ }^{2}$}

\subsection{Introduction}

Long-term investment and planning in the power sector need to consider uncertainties in future hazard trends. One such uncertainty is the potential impact of climate change on hurricane hazard. The Fifth Assessment Report of the Intergovernmental Panel on Climate Change (IPCC) noted the variation in weather patterns and projected an increase in the intensity of storms (Zamuda et al., 2013; IPCC, 2013). With the potential increase in the intensity of hurricanes in the long-term due to climate change, the cost of damage to distribution system is expected to rise. Consequently, a report by the members of the electric utilities project of the World Business Council on Sustainable Development (WBCSD) called on all utility companies to build expertise in analyzing climate information to better understand climate change related risks and to develop adaptation and resiliency strategies to cope with such risks (WBCSD, 2014).

The potential impact of climate change is magnified when aging of power distribution infrastructure is considered, which has been determined to be one of the main issues facing the power system in the United States (U.S.) (ASCE, 2013). With the inherent uncertainty in the prediction of variation in weather patterns as well as the time-dependent strength of infrastructure components, a better understanding of the potential varied risks is essential for informed decision making. Therefore, there is a need to investigate the possible impact of climate change on power distribution systems and come up with economically feasible adaptation strategies.

\footnotetext{
${ }^{2}$ A version of this chapter was previously published in Journal of Infrastructure Systems and is re-used herein with permission from ASCE. The permission is presented in Appendix D.
} 
Bjarnadottir et al. (2013) and (2014) presented a probabilistic framework for evaluating the potential impact of climate change on hurricane risk assessment of power distribution poles and proposed various adaptation strategies. The framework incorporated probabilistic hurricane analysis, age-dependent fragility of poles, and life cycle cost analysis. The effect of climate change was modeled by assuming a linear change in wind speed of $-5 \%$ to $25 \%$ over a 100-year period. Francis et al. (2011) presented a hybrid economic input-output life cycle cost analysis method for evaluating the cost-effectiveness of climate change adaptation strategies of distribution systems. Failure of distribution poles, spans, and padmounted transformers was considered in the study. The effect of climate change was modeled through the use of count regression analysis and data mining techniques that describe the relationship between climate variability and North Atlantic tropical cyclone counts in the U.S.

While the above studies constitute great strides in studying the impact of climate change on power distribution systems, they are limited to component-level risk assessment. A more informed decision on the effectiveness of adaptation strategies can be made when the reliability of the entire distribution system is evaluated. This will allow determining the parts of the system with greater impact on reliability which ought to be strengthened.

In this chapter, the framework of Chapter 4 is extended to integrate the potential impact of climate change on hurricane patterns. A hurricane simulation model is adopted to enable the variation in both intensity and frequency to be considered. Adaptation strategies are proposed and their cost-effectiveness investigated. Micropolis power distribution system is used to demonstrate the framework. Class 4 and class 5 southern pine poles as designed in Chapter 4 are used for the three-phase and single-phase lines, respectively. All poles are assumed to be new at the start of the system reliability analysis. 


\subsection{Hurricane Risk Assessment}

Hurricane simulation is the most widely used method of hurricane risk analysis for design and assessment of structures and infrastructure (Vickery et al., 2000b). Hurricane simulation models involve using site-specific statistics of key hurricane parameters and Monte Carlo simulation for assessing hurricane hazard level. Conducting hurricane simulation is the best way to account for the potential effect of climate change on hurricane hazard as it will allow for the modification of parameters such as frequency and intensity within the simulation model.

The distribution and statistical moments of the basic parameters needed for hurricane simulation are obtained from records of historical hurricanes. The most complete and reliable historical data for north Atlantic hurricanes is provided by the North Atlantic Hurricane Data Base (HURDAT) and compiled by the Oceanographic and Meteorological Laboratory at National Oceanic \& Atmospheric Administration (NOAA, 2015a).

The current HURDAT data contains details for hurricanes from 1851 - 2013. Key parameters provided in the HURDAT data include approximate landing position, maximum sustained (1-minute) surface wind speed, Saffir-Simpson category, central pressure, and affected states. The complete hurricane simulation method is explained below.

\subsubsection{Hurricane Simulation Model}

The various parameters, their statistics, and how they are determined are explained below based on the model developed by Xu and Brown (2008a). 


\section{i. Annual hurricane frequency}

The number of hurricanes for any given year can be simulated according to a Poisson distribution (Chen et al., 2004; Huang et al., 2001a; Xu \& Brown, 2008b; Mudd et al., 2014a). The Poisson distribution is modeled as:

$$
f(x)=\frac{\lambda^{x}}{x !} e^{-\lambda} ; x=0,1,2, \ldots
$$

where $x$ is the number of hurricanes per year, $\lambda$ is the average number of hurricanes in a given year computed from historical records, and $f(x)$ is the probability of $x$ hurricanes in a given year.

\section{ii. Landfall position}

The landing position of a simulated hurricane is usually expressed in latitude and longitude. The landing position is assigned based on the distribution of historical hurricanes landing in a specific area by assuming uniform distribution or by dividing the coastline into bins as suggested by Xu and Brown (2008a) and Huang et al. (2001a).

\section{iii. $\quad$ Approach angle}

The approach angle shows the direction a hurricane heads to after making landfall. The approach angle is measured with North as 0 degrees. Based on historical data, the approach angle is modeled with a bi-normal distribution (Xu \& Brown, 2008a; Toth \& Szentimrey, 1990):

$$
f(\theta)=\frac{a_{1}}{\sqrt{2 \pi} \sigma_{1}} \exp \left[-\frac{1}{2}\left(\frac{\theta-\mu_{1}}{\sigma_{1}}\right)^{2}\right]+\frac{\left(1-a_{1}\right)}{\sqrt{2 \pi} \sigma_{2}} \exp \left[-\frac{1}{2}\left(\frac{\theta-\mu_{2}}{\sigma_{2}}\right)^{2}\right]
$$


where $\mu_{1}$ and $\mu_{2}$ are the means, $\sigma_{1}$ and $\sigma_{2}$ are the standard deviations of the approach angle, and $a_{1}$ is the weighting factor. The landing position and approach angle determine the path of a hurricane after landfall. Xu and Brown (2008a) demonstrated that it is reasonable to assume hurricanes travel along a straight path in Florida due to the narrow shape of the state.

\section{iv. Translation velocity}

Translation velocity is the forward speed of the hurricane. It can be modeled as a lognormal distribution as (Vickery \& Twisdale, 1995a; Georgiou et al., 1983; Brown, 2009; Huang et al., 2001a):

$$
f(c)=\frac{1}{\sqrt{2 \pi} c \zeta} \exp \left[-\frac{1}{2}\left(\frac{\ln c-\lambda}{\zeta}\right)\right]
$$

where $c$ is the translation velocity, $\lambda$ is the logarithmic mean, and $\zeta$ is the logarithmic standard deviation. The translation velocity is assumed to be constant after landfall (Xu \& Brown, 2008a).

\section{v. Central pressure difference}

This is the difference between atmospheric pressure at the center and at the periphery of the hurricane. The central pressure difference is modeled from historical data using the Weibull distribution (Georgiou et al., 1983; Vickery \& Twisdale, 1995a; Huang et al., 2001b; Xu \& Brown, 2008a) as:

$$
f_{v}(v)=\frac{\alpha}{u}\left(\frac{\Delta p}{u}\right)^{\alpha-1} \exp \left[-\left(\frac{\Delta p}{u}\right)^{\alpha}\right]
$$

where $\Delta p$ is the central pressure difference, and $u$ and $\alpha$ are the parameters of the Weibull distribution determined from historical data. 
The rise in central pressure (which results in weakening of intensity) of the hurricane after landfall is modeled as (Xu \& Brown, 2008a; Huang et al., 2001a; Vickery \& Twisdale, 1995b):

$$
\Delta p(t)=\Delta p_{o} \exp (-a t)
$$

where $\Delta p(t)$ is the central pressure difference at time $t, \Delta p_{o}$ is the central pressure difference at landfall, $a$ is a decay constant. For Florida, $a$ is given by (Vickery $\&$ Twisdale, 1995b):

$$
a=0.006+0.00046 \cdot \Delta p_{o}+\varepsilon
$$

where $\varepsilon$ is an error term that is normally distributed with a mean of zero and standard deviation of 0.025 .

\section{vii. Maximum wind speed at landfall}

The maximum wind speed at landfall is proportionally assigned based on the simulated central pressure difference as suggested by Xu and Brown (2008a). Table 5.1 shows the relationship between hurricane category, minimum central pressure, and maximum sustained wind speed (NOAA, 2015c). 
Table 5.1 The Saffir-Simpson hurricane scale

\begin{tabular}{ccc}
\hline \hline Hurricane Category & $\begin{array}{c}\text { Saffir-Simpson wind } \\
\text { speed range }(\mathrm{mph})\end{array}$ & $\begin{array}{c}\text { Central pressure range } \\
(\mathrm{mbar})\end{array}$ \\
\hline 1 & $74-95$ & $\geq 980$ \\
2 & $96-110$ & $965-979$ \\
3 & $111-130$ & $945-964$ \\
4 & $131-155$ & $920-944$ \\
5 & $>155$ & $<920$ \\
\hline
\end{tabular}

viii. Maximum wind speed decay

Hurricane wind speed decays after landfall due to friction by land mass and reduction in storm's moisture. The most widely used speed decay model is known as KD95 developed by Kaplan and DeMaria (1995). The model is based on the assumption that hurricane wind speeds decay at a rate proportional to their landfall intensity and decay exponentially over land. The wind speed at any given time is given by (Kaplan \& DeMaria, 1995; DeMaria et al., 2006):

$$
V(t)=V_{b}+\left(R V_{0}-V_{b}\right) e^{-\alpha t}
$$

where $R$ is a sea-land wind speed reduction factor with a value of $0.9, V_{b}=13.75 \mathrm{~m} / \mathrm{s}$ and is a constant "background" intensity, $V_{0}$ is the maximum sustained 1-min wind speed at landfall, and $\alpha=0.095 \mathrm{~h}^{-1}$ which is a decay constant.

\section{ix. $\quad$ Radius to maximum winds}

This describes the range of the most intensive hurricane wind speed. FEMA (2005) developed the equation for the radius of maximum winds $R_{\max }$ as: 


$$
\ln R_{\max }=2.556-0.000050255 \Delta p^{2}+0.042243032 \varphi
$$

where $\varphi$ is the storm latitude and $\Delta p$ is the central pressure difference. A similar model can also be found in Vickery and Twisdale (1995a).

\section{x. Radial wind field model}

The variation of wind speed from hurricane eye to periphery is modeled by a radial wind field model. The gradient wind speed $\left(V_{G}\right)$ at any location at any time instant is given by (Holland, 1980; Vickery et al., 2009a):

$$
V_{G}=\left[\left(\frac{R_{\max }}{r}\right)^{B}\left(\frac{B \Delta p \exp \left[-\left(\frac{R_{\max }}{r}\right)^{B}\right]}{\rho}\right)+\frac{r^{2} f^{2}}{4}\right]^{1 / 2}-\frac{r f}{2}
$$

where $R_{\max }$ is the radius to maximum wind speed, $r$ is the distance from hurricane eye to point of interest, $B$ is the Holland parameter, $\Delta p$ is the central pressure difference, $\rho$ is air density $\left(1.15 \mathrm{~kg} / \mathrm{m}^{3}\right)$, and $f$ is the Coriolis parameter. The Holland parameter, $B$, can be determined from (Holland, 1980):

$$
B=\frac{\rho e\left(V_{m}^{2}\right)}{\Delta p}
$$

where $V_{m}$ is the maximum wind speed, $e$ is the base of natural logarithm, $\Delta p$ is the central pressure difference, $\rho$ is air density.

The gradient wind speed in Equation (5.9) needs to be converted to surface wind speed to assess the performance of infrastructure systems. The conversion factor can range from 0.8 for weaker storms to 0.86 for intense storms (Vickery et al., 2000a; Vickery et al., 2009a). Furthermore, the surface wind speed needs to be converted to 3-sec gust wind speed as 
structural damage is more related to peak gust wind speed which is the maximum instantaneous wind speed. Therefore, a gust factor is needed to convert the surface wind speed. The gust model developed by Engineering Sciences Data Unit (ESDU, 1982, 1983) has been shown to be adequate for modeling gust factors (Xu \& Brown, 2008a). Xu and Brown (2008a) conducted a 1000-year simulation to estimate the 3 -sec gust factor using the ESDU model and found that the distribution of the calculated values of the factor is highly concentrated around 1.287 with a standard deviation of 0.002 . This value has been adopted for use in this research.

The steps for the hurricane simulation are shown in Figure 5.1. The simulation is carried out for 200,000 hurricane seasons. 


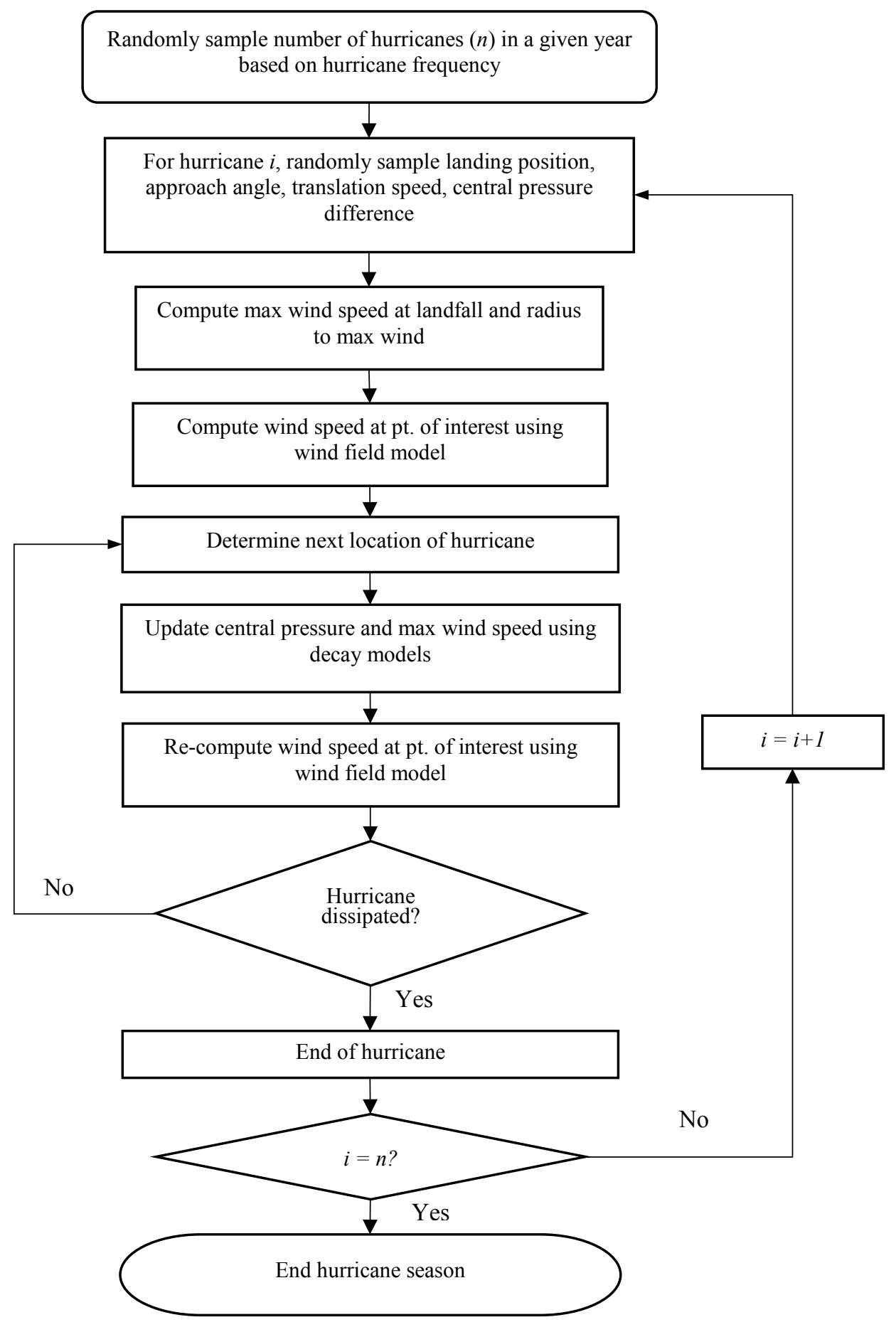

Figure 5.1 Hurricane simulation model flowchart 
The required parameters for the hurricane simulation can be obtained from historical records compiled in HURDAT. For Florida, which is the selected location for this research, historical hurricane record in HURDAT are provided for four different regions namely southeast, southwest, northeast, and northwest. The selected study area is assumed to be located in southeast Florida. Historical hurricanes that made landfall in Florida are summarized in Table 5.2 (NOAA, 2015a; Xu \& Brown, 2008a).

Table 5.2 Hurricane occurrence in different parts of Florida from $1851-2014$

\begin{tabular}{ccccc}
\hline \hline \multirow{2}{*}{$\begin{array}{c}\text { Hurricane } \\
\text { category }\end{array}$} & \multicolumn{4}{c}{ No. of occurrence } \\
\cline { 2 - 5 } & Southeast & Northeast & Southwest & Northwest \\
\hline 1 & 8 & 3 & 12 & 20 \\
2 & 9 & 3 & 7 & 13 \\
3 & 10 & 0 & 6 & 13 \\
4 & 3 & 0 & 3 & 0 \\
5 & 1 & 0 & 1 & 0 \\
Total & $\mathbf{3 1}$ & $\mathbf{6}$ & $\mathbf{2 9}$ & $\mathbf{4 6}$ \\
\hline
\end{tabular}

The statistical parameters of the variables computed from historical records are given in Table 5.3 for different regions of Florida (Xu \& Brown, 2008b; Huang et al., 2001a).

Table 5.3 Statistics of hurricane simulation parameters for different regions of Florida

\begin{tabular}{|c|c|c|c|c|c|}
\hline \multirow[t]{2}{*}{ Variable } & \multirow[t]{2}{*}{ Distribution } & \multicolumn{4}{|c|}{ Distribution parameters } \\
\hline & & Southeast & Northeast & Southwest & Northwest \\
\hline Annual frequency, $\lambda$ & Poisson & 0.2 & 0.039 & 0.1871 & 0.297 \\
\hline $\begin{array}{l}\text { Approach angle, } \theta \\
\text { (degrees) }\end{array}$ & Bi-normal & $\begin{array}{c}\mu_{1}=310 \\
\sigma_{1}=30 \\
\mu_{2}=35 \\
\sigma_{2}=15 \\
a_{1}=0.9\end{array}$ & $\begin{array}{c}\mu_{1}=345 \\
\sigma_{1}=5 \\
\mu_{2}=285 \\
\sigma_{2}=10 \\
a_{1}=0.5\end{array}$ & $\begin{array}{c}\mu_{1}=40 \\
\sigma_{1}=25 \\
\mu_{2}=300 \\
\sigma_{2}=30 \\
a_{1}=0.63\end{array}$ & $\begin{array}{c}\mu_{1}=35 \\
\sigma_{1}=25 \\
\mu_{2}=295 \\
\sigma_{2}=40 \\
a_{1}=0.5\end{array}$ \\
\hline $\begin{array}{l}\text { Central pressure } \\
\text { difference }\end{array}$ & Weibull & \multicolumn{2}{|c|}{$\begin{array}{c}u=64.831 \\
\alpha=3.465\end{array}$} & \multicolumn{2}{|c|}{$\begin{array}{c}u=42.751 \\
\alpha=3.929\end{array}$} \\
\hline Translation velocity & Lognormal & \multicolumn{4}{|c|}{$\begin{array}{c}\lambda=2.3-0.00275 \theta^{*} \\
\zeta=0.3\end{array}$} \\
\hline
\end{tabular}

*Parameters were found to be correlated with approach angle (Xu \& Brown, 2008b, 2008a) 
Figure 5.2 shows the spatial variation of a simulated hurricane at landfall. Note that in the model above, only hurricanes that landed in Florida are considered for evaluating the required parameters and the model start at the point of landfall rather than at locations in the Atlantic Ocean or the Gulf of Mexico. Furthermore, both central pressure filling rate and maximum wind speed decay discussed in the hurricane simulation model above will be used as suggested by $\mathrm{Xu}$ and Brown (2008a) since there is no empirical equation relating the central pressure and wind speed after landfall.

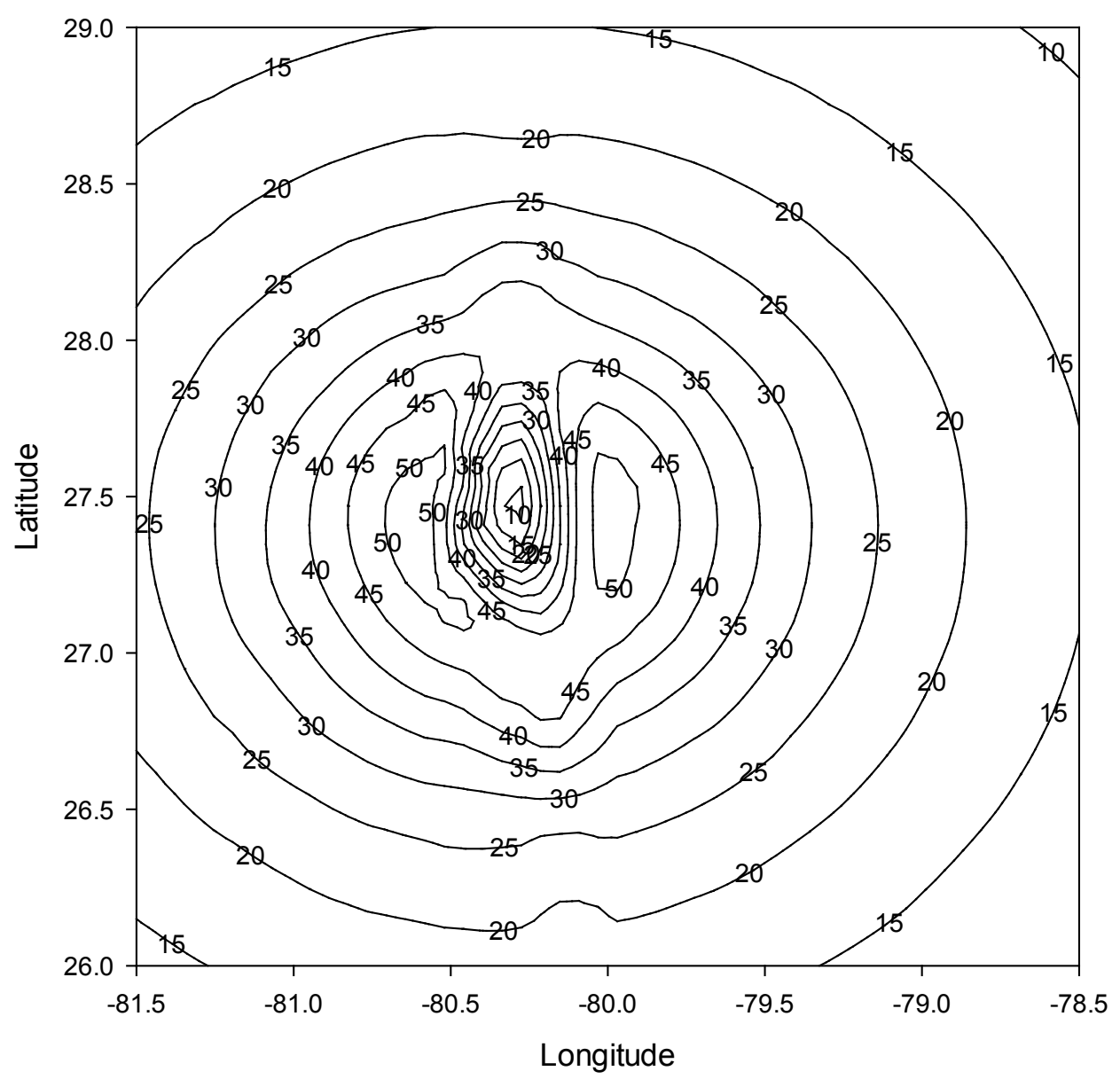

Figure 5.2 Surface wind speed variation for a simulated hurricane 


\subsubsection{Hurricane Simulation Model Validation}

The simulation model described above is used to estimate the annual maximum hurricane wind speed for 200,000 years at a particular location $\left(27.6^{\circ} \mathrm{N}, 80.4^{\circ} \mathrm{W}\right)$ on the east coast of Florida which is the assumed location of the case study to be discussed later. The maximum annual hurricane wind speed can be modeled by an extreme value (EV) distribution (Yeo et al., 2014; Coles \& Simiu, 2003; Jagger et al., 2001; Jagger \& Elsner, 2006). All the three types of EV distributions, namely Gumbel, Fréchet, and reversed Weibull (or simply Weibull), were fitted to the data as shown in

Figure 5.3. It can be seen from the figure that Fréchet distribution provides the best fit for the data followed by Weibull distribution. On the other hand, Gumbel distribution does not seem to fit the data. Hence it is not considered for further analysis.

The scale and shape parameters of the Weibull distribution determined using the maximum likelihood method in MATLAB are 26.24 and 1.88 respectively. The shape, scale, and location parameters of the Fréchet distribution are found to be $0.19,8.66$, and 16.12, respectively. 


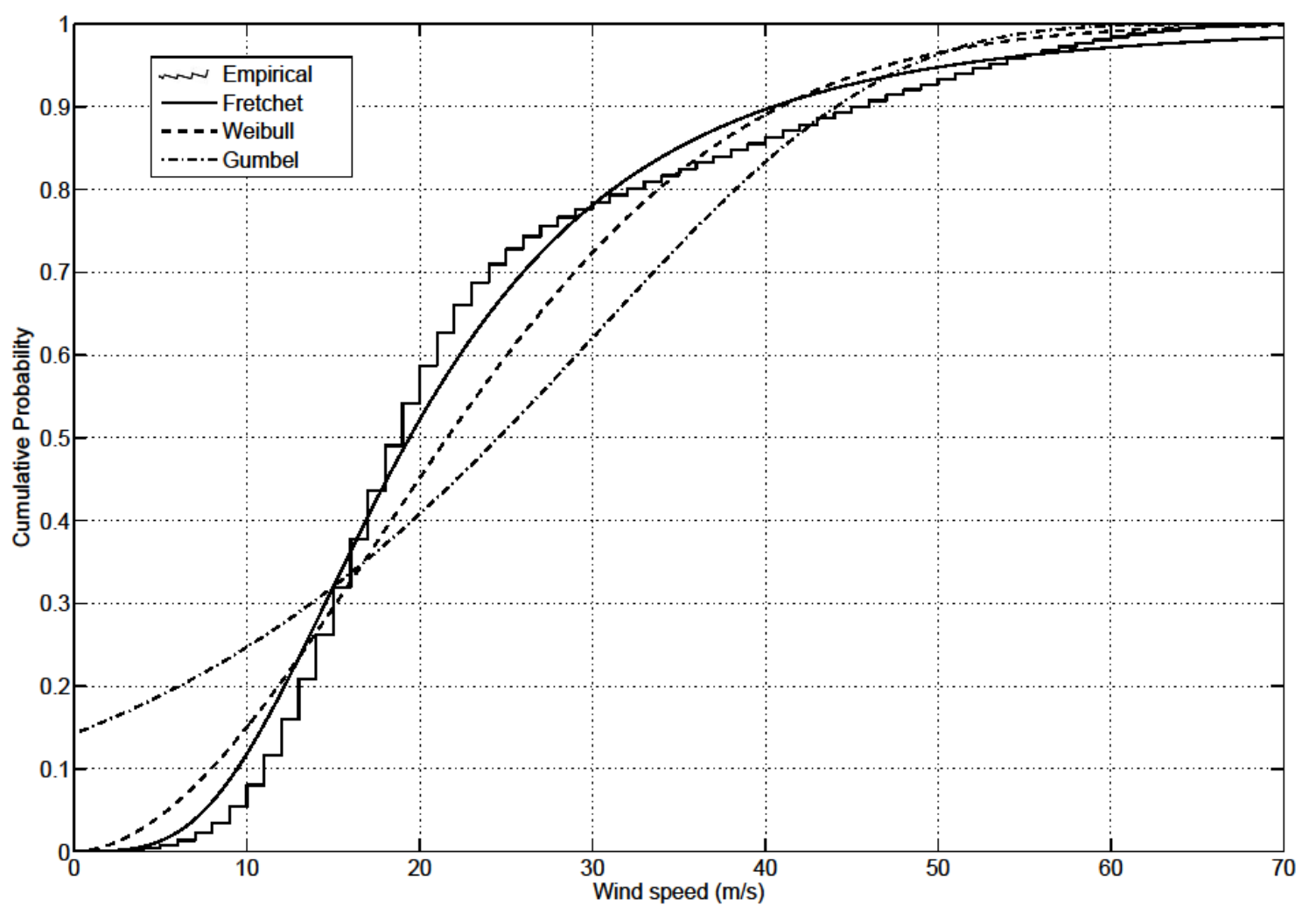

Figure 5.3 CDF of max annual hurricane wind speed with fitted distributions

To validate the hurricane simulation model, wind speeds corresponding to different mean recurrence intervals (MRI) for the chosen location are calculated and compared to values in ASCE-7 (2010) as shown in Table 5.4 and plotted as hazard curves in Figure 5.4. The wind speeds corresponding to different return periods can be obtained from the fact that, assuming independent maximum annual hurricane wind speeds, the probability of exceeding $N$-year MRI wind speed in $t$ years is given by:

$$
F_{t}(v>V)=1-\left[1-\left(\frac{1}{\mathrm{~N}}\right)\right]^{t}
$$

From Table 5.4, it can be seen that the wind speeds for different MRIs predicted by the Weibull distribution are very close to those obtained from ASCE-7 (2010). For the Fréchet distribution, however, even though it is a better fit to the data than Weibull distribution, it 
resulted in unrealistically high wind speeds, especially for larger MRIs. This anomaly has been documented in the literature which is why the Fréchet distribution is not recommended for modeling maximum annual hurricane wind speeds (Yeo et al., 2014). The Weibull distribution is therefore chosen for modeling the wind speed data. Note that the ASCE-7 (2010) wind speeds corresponding to different MRI were extracted from ATC (2015).

Table 5.4 Comparison of wind speeds corresponding to different return periods

\begin{tabular}{llll}
\hline \hline MRI (years) & $\begin{array}{l}\text { ASCE-7 (2010) } \\
\text { values }(\mathrm{m} / \mathrm{s})\end{array}$ & $\begin{array}{l}\text { Values predicted } \\
\text { by Weibull }(\mathrm{m} / \mathrm{s})\end{array}$ & $\begin{array}{l}\text { Values predicted } \\
\text { by Fréchet }(\mathrm{m} / \mathrm{s})\end{array}$ \\
\hline 10 & 39 & 41 & 40 \\
25 & 47 & 49 & 54 \\
50 & 52 & 54 & 66 \\
100 & 58 & 59 & 80 \\
300 & 65 & 67 & 108 \\
700 & 70 & 71 & 129 \\
1700 & 75 & 76 & 157 \\
\hline \hline
\end{tabular}




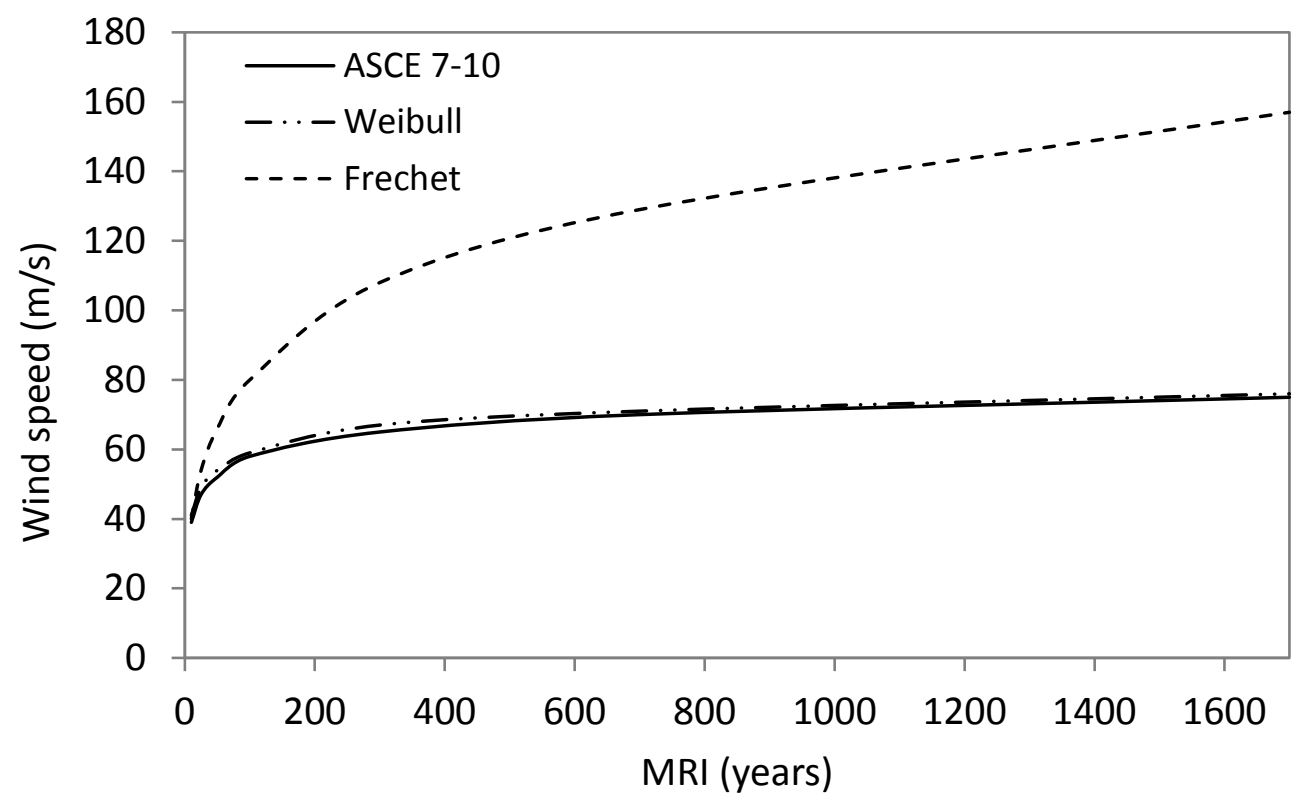

Figure 5.4 Wind speeds comparison

\subsection{Non-stationary Hurricane Wind Model due to Climate Change}

The biggest source of uncertainty for future climate prediction is the level of management of greenhouse gas emissions by society (IPCC, 2013). Consequently, studies on the effect of climate change on infrastructure are approached by considering various future climate change scenarios. Different climate change scenarios are adopted to investigate what might happen in the future under a particular assumption. These scenarios provide starting points for examining an uncertain future and evaluating the effects such scenarios might have on civil infrastructure systems.

The most recent climate change scenarios proposed by IPCC (2013) are based on greenhouse gas concentration pathways ( $\mathrm{CPs}$ ) which are determined by their radiative forcing at the end of the $21^{\text {st }}$ century. Radiative forcing is defined by ASCE (2015) as the "change in the balance between incoming and outgoing radiation caused by changes in greenhouse gas concentrations and other atmospheric constituents, while other aspects of the atmosphere are held constant". Four Representative Concentration Pathways (RCPs) 
were produced by IPCC (2013) that correspond to radiative forcing levels of 8.5, 6.0, 4.5, and 2.6 watts $/ \mathrm{m}^{2}$ and are termed RCP 8.5, RCP 6.0, RCP 4.5, and RCP 2.6, respectively. It might be of interest to note that the forcing level in 2005 was estimated to be 1.6 watts $/ \mathrm{m}^{2}$ (Bernstein et al., 2007). Older climate change scenarios method used by the IPCC is the SRES scenarios details of which can be found in IPCC (2000).

The effect of the above scenarios on the frequency and intensity of hurricanes has been a subject of much discussion. Bender et al. (2010) modeled the effect of one of the SRES climate change scenarios on the frequency of Atlantic hurricanes and concluded that the frequency of the most intense hurricanes (category 3-5) is expected to increase through the year 2100. Knutson et al. (2010) concluded that the global frequency of tropical cyclones will either decrease or remain unchanged with the authors predicting a decrease between 6 to $-34 \%$. Projections for individual basins were reported to be up to $\pm 50 \%$ with very low confidence. It was however reported that the frequency of the most intense storms will "more likely than not increase by a substantially larger percentage in some basins". Knutson et al. (2010) also projected the intensity of tropical cyclones, measured as mean maximum wind speed, to increase between $+2 \%$ to $+11 \%$ globally.

Some studies have also shown a link between an increase in sea surface temperatures (SST) and hurricane frequency (Mann \& Emanuel, 2006; Mann et al., 2007; Elsner et al., 2008). Mudd et al. (2014a) used the Community Earth System Model (CESM) of the National Center for Atmospheric Research (NCAR) to model the increase in sea surface temperature (SST) at the year 2100 under RCP 8.5 scenario. The resulting SST was then used in a hurricane simulation model that takes into account changes in SSTs. The authors concluded that under future climate scenario RCP 8.5 in 2100, the maximum wind speed associated with Atlantic hurricanes is expected to increase.

Staid et al. (2014) reported the bounding range for change in hurricane intensity from existing literature to be between $-20 \%$ to $+40 \%$. Landsea et al. (2010) on the other hand reported the range of future hurricane frequency to be between $-30 \%$ to $+35 \%$. Based on 
these estimates and the above literature review, the following climate change scenarios (CCSs) at the end of the $21^{\text {st }}$ century are assumed for this study:

i. CCS1: no change in frequency, $+20 \%$ change in intensity

ii. CCS2: $+20 \%$ change in frequency, no change in intensity

iii. CCS3: $-20 \%$ change in frequency, $+20 \%$ change in intensity

iv. CCS4: $+20 \%$ change in frequency, $+20 \%$ change in intensity

v. CCS5: $+35 \%$ change in frequency, $+40 \%$ change in intensity

vi. CCS6: $+35 \%$ change in frequency, $-20 \%$ change in intensity

The change in frequency and intensity from the present time to the end of the $21^{\text {st }}$ century is assumed to be linear as suggested by Stewart et al. (2014). It is however noted that considerable interdecadal and intradecadal variations can occur in the trend (Stewart et al., 2014; Mudd et al., 2014b). The above climate change scenarios are incorporated into the hurricane simulation model by altering certain parameters. For frequency variation, the parameter of the Poisson distribution, $\lambda$, is altered. For intensity variation, the randomly sampled central pressure difference at landfall is increased or decreased by a percentage depending on the climate change scenario being considered.

The hazard curves for the chosen location on the east coast of Florida are plotted in Figure 5.5 for the baseline scenario (no change) and the six CCSs above. It can be noted from the figure that changes in intensity have higher effect on wind speeds than changes in frequency. For example, CCS1 (no change in frequency, $+20 \%$ change in intensity) results in higher wind speeds at all return periods than CCS2 $(+20 \%$ change in frequency, no change in intensity). The same conclusion can be drawn by comparing CCS2 and CCS3. Among the six scenarios, only CCS6 $(+35 \%$ change in frequency, $-20 \%$ change in intensity) resulted in a decrease in wind speed at all return periods despite $35 \%$ increase in frequency. 


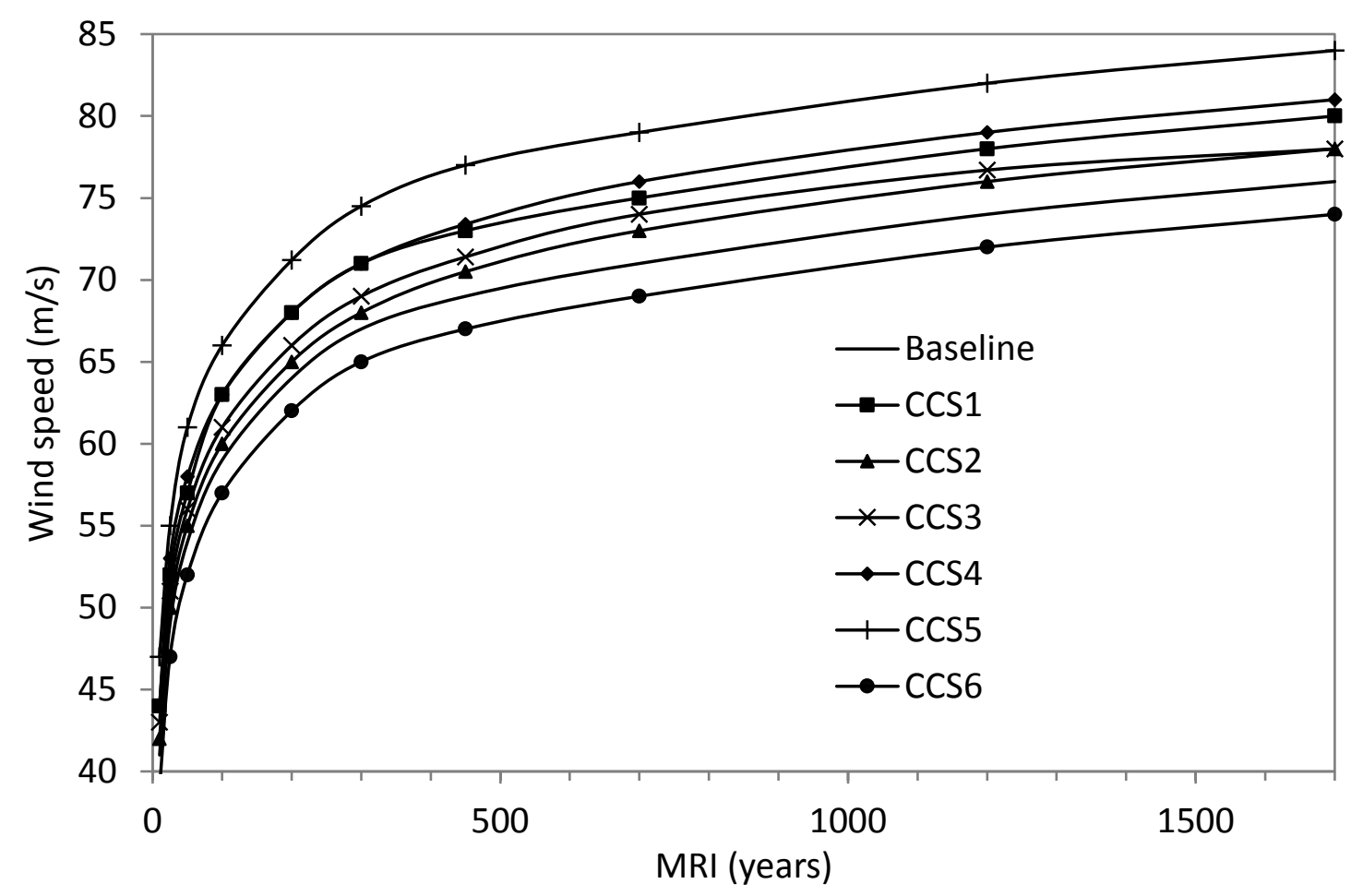

Figure 5.5 Hazard curves for different climate scenarios

\subsection{System Reliability Results}

The system reliability results for the no change scenario (baseline) and the six selected climate scenarios are plotted in Figure 5.6 for the period 2010 to 2100. It can be seen that the pattern of change in system reliability follows the pattern in Figure 5.5 as expected. All scenarios except CCS6 ( $+35 \%$ change in frequency, $-20 \%$ change in intensity) resulted in a decrease in system reliability over the years. CCS2 $(+20 \%$ change in frequency, no change in intensity) and CCS5 ( $+35 \%$ change in frequency, $+40 \%$ change in intensity) resulted in the least and highest decrease in reliability over time, respectively. For example, at 2080 , the system reliability decreased from $67 \%$ for the baseline case to $63 \%$ and $40 \%$ for CCS2 and CCS5, respectively. This implies that climate change can have a significant effect on system reliability over time. 
According to NESC (2002), wood poles should be replaced or reinforced when their strength falls below $67 \%$ of the initial strength. This will happen when the poles are around 70 years old as seen in Figure 4.5 in the previous chapter. Consequently, in this case, the poles in the system that survived from 2010 to 2080 will be replaced as part of a periodic maintenance program which is not considered in Figure 5.6. This will, however, be reflected in the LCCA section when pole replacement due to both periodic maintenance and wind-induced failure is considered.

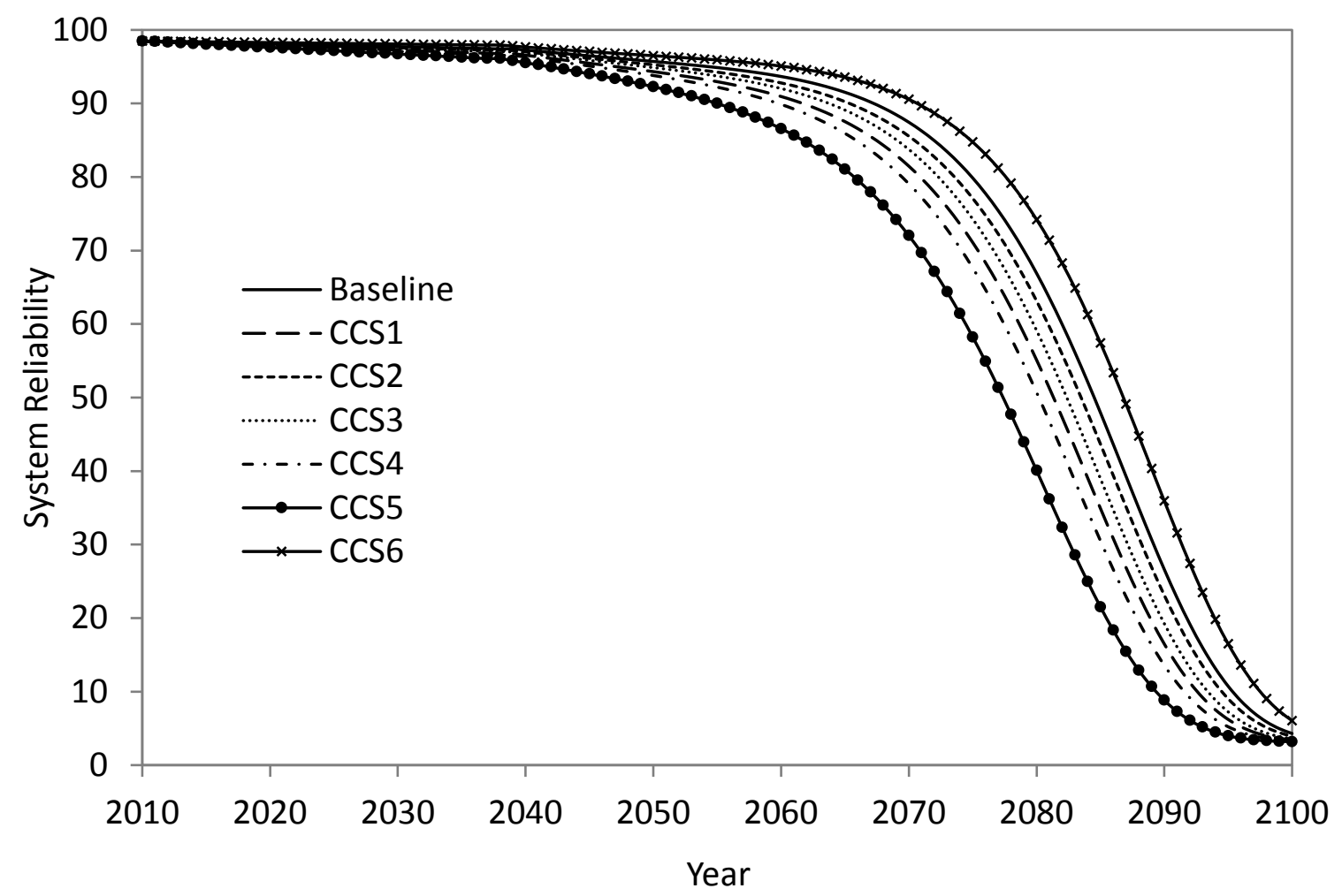

Figure 5.6 System reliability results for different climate scenarios

\subsection{Adaptation Strategies}

Increase in hurricane wind speed due to climate change which consequently causes a decrease in reliability of the system as shown above warrants some form of climate change 
adaptation strategies to be implemented. Three climate change scenarios, CCS2 $(+20 \%$ change in frequency, no change in intensity), CCS4 ( $+20 \%$ change in frequency, $+20 \%$ change in intensity), and CCS5 ( $+35 \%$ change in frequency, $+40 \%$ change in intensity) are selected to demonstrate the life cycle cost analysis procedure for comparing various climate change adaptation strategies. CCS4 and CCS5 are chosen because they resulted in the highest decrease in system reliability over time. CCS2 is chosen because it resulted in only a slight decrease in system reliability. Therefore, it will give an indication of whether adaptation strategies are cost-effective when the level of climate change is very small. Two adaptation strategies are proposed below.

Strategy 1: Strengthening entire system

In this strategy, all new distribution systems are to be constructed with poles that are one class higher than the required pole class determined using the design method recommended in ASCE-111 (2006). The adaptation cost is then the additional cost needed to use stronger poles instead of the poles that have been shown to be sufficient during the design. The distribution system model in this research is assumed to be strengthened during construction in 2010 .

Strategy 2: Strengthening parts of a system

This strategy is similar to Strategy 1 except that here, only the main feeder lines, which deliver electric power to the laterals, are replaced with poles that are one class higher. The improvement in system reliability when the two adaptation strategies are applied to CCS5 is shown in Figure 5.7. Strategy 1 results in higher improvement in system reliability as expected. At 2080 for example, the system reliability is $40 \%, 65 \%$, and $56 \%$ for the unhardened system, strategy 1 , and strategy 2 , respectively. Note that pole replacement is not considered in the plot of Figure 5.7. 


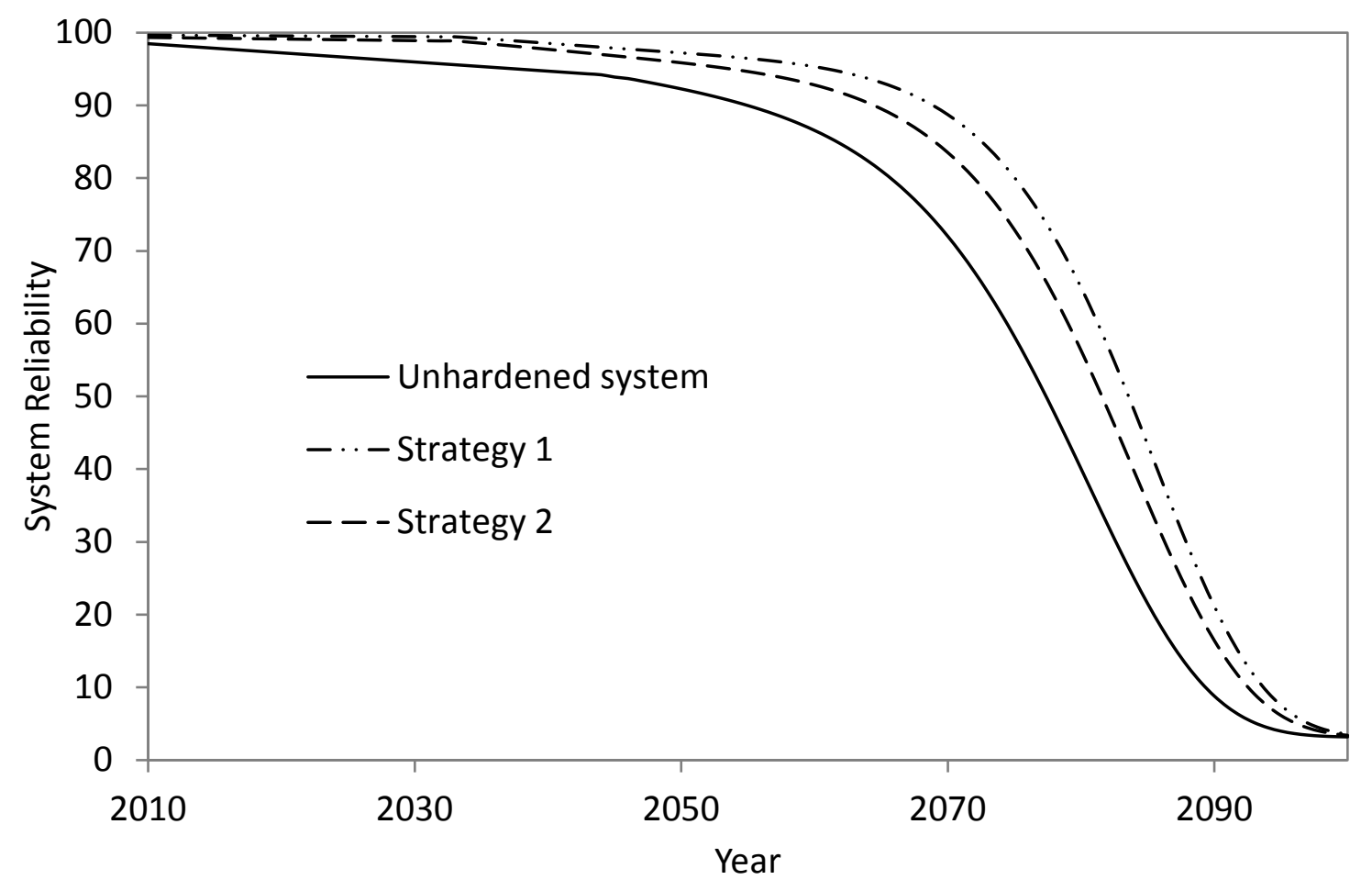

Figure 5.7 System reliability improvement for CCS5

\subsection{Life Cycle Cost Analysis Result}

The LCCA is carried out for the years from 2010 to 2100 . The LCCA is first performed to compare the baseline case and the three selected climate change scenarios and the results are shown in Table 5.5 and Figure 5.8. It can be seen that the LCC increases as the severity of climate change increases. In this case, the LCC increased by $2 \%, 6 \%$, and $12 \%$ for CCS2, CCS4, and CCS5, respectively. 
Table 5.5 LCCA results for different scenarios $(\$ 1,000 \mathrm{~s})$

\begin{tabular}{lllll}
\hline \hline Cost category & $\begin{array}{l}\text { No climate } \\
\text { change }\end{array}$ & CCS2 & CCS4 & CCS5 \\
\hline Maintenance cost, $C_{m}$ & 1,871 & 1,867 & 1,857 & 1,846 \\
Repair cost, $C_{r}$ & 432 & 448 & 489 & 541 \\
Revenue loss, $C_{v}$ & 6 & 6 & 7 & 7 \\
Societal Economic loss, $C_{e}$ & 1,600 & 1,665 & 1,802 & 1,987 \\
\hline Total LCC & $\mathbf{3 , 9 0 9}$ & $\mathbf{3 , 9 8 6}$ & $\mathbf{4 , 1 5 5}$ & $\mathbf{4 , 3 8 1}$ \\
\hline Percentage Increase in LCC & - & $\mathbf{2}$ & $\mathbf{6}$ & $\mathbf{1 2}$ \\
$\mathbf{( \% )}$ & & & & \\
\hline \hline
\end{tabular}

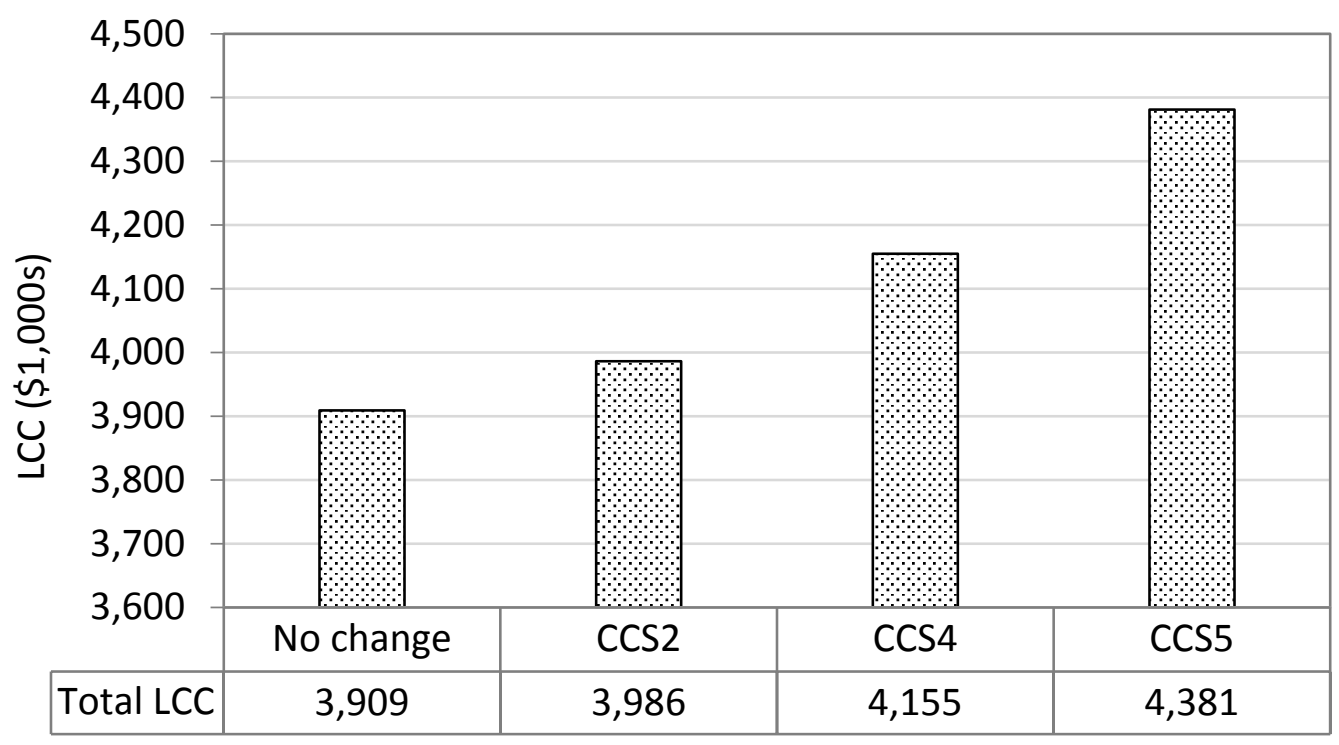

Figure 5.8 LCC results for different climate scenarios

The results of the LCCA for the selected climate change scenarios and the adaptation strategies are shown in Table 5.6, Table 5.7, and Table 5.8. For all the three scenarios, the adaptation strategies lower the repair cost, revenue loss, and societal economic losses. The total LCC for all three cases is also plotted in Figure 5.9 and it can be observed that strategy 1 results in lower LCC in all cases even though it has higher adaptation cost than strategy 2. 
Table 5.6 LCCA results for CCS2 scenario $(\$ 1,000 \mathrm{~s})$

\begin{tabular}{llll}
\hline \hline Cost category & Unhardened system & Strategy 1 & Strategy 2 \\
\hline Adaptation cost, $C_{M}$ & - & 29 & 9 \\
Maintenance cost, $C_{m}$ & 1,867 & 1,897 & 1,874 \\
Repair cost, $C_{r}$ & 448 & 337 & 420 \\
Revenue loss, $C_{v}$ & 6 & 5 & 5 \\
Societal Economic loss, $C_{e}$ & 1,665 & 1,211 & 1,236 \\
\hline Total LCC & $\mathbf{3 , 9 8 6}$ & $\mathbf{3 , 4 7 9}$ & $\mathbf{3 , 5 4 4}$ \\
Total LCC w/o $C_{\boldsymbol{e}}$ & $\mathbf{2 , 3 2 1}$ & $\mathbf{2 , 2 6 8}$ & $\mathbf{2 , 3 0 8}$ \\
\hline Net benefit & - & $\mathbf{5 0 7}$ & $\mathbf{4 4 2}$ \\
Net benefit w/o $C_{\boldsymbol{e}}$ & - & $\mathbf{5 3}$ & $\mathbf{1 3}$ \\
\hline \hline
\end{tabular}

Table 5.7 LCCA results for CCS4 scenario $(\$ 1,000 \mathrm{~s})$

\begin{tabular}{llll}
\hline \hline Cost category & Unhardened system & Strategy 1 & Strategy 2 \\
\hline Adaptation cost, $C_{M}$ & - & 29 & 9 \\
Maintenance cost, $C_{m}$ & 1,857 & 1,889 & 1,864 \\
Repair cost, $C_{r}$ & 489 & 420 & 459 \\
Revenue loss, $C_{v}$ & 7 & 5 & 5 \\
Societal Economic loss, $C_{e}$ & 1,802 & 1,312 & 1,339 \\
\hline Total LCC & $\mathbf{4 , 1 5 5}$ & $\mathbf{3 , 6 5 5}$ & $\mathbf{3 , 6 7 6}$ \\
Total LCC w/o $C_{\boldsymbol{e}}$ & $\mathbf{2 , 3 5 3}$ & $\mathbf{2 , 3 4 3}$ & $\mathbf{2 , 3 3 7}$ \\
\hline Net benefit & - & $\mathbf{5 0 0}$ & $\mathbf{4 7 9}$ \\
Net benefit w/o $C_{\boldsymbol{e}}$ & - & $\mathbf{1 0}$ & $\mathbf{1 6}$ \\
\hline \hline
\end{tabular}

Table 5.8 LCCA results for CCS5 scenario $(\$ 1,000 \mathrm{~s})$

\begin{tabular}{llll}
\hline \hline Cost category & Unhardened system & Strategy 1 & Strategy 2 \\
\hline Adaptation cost, $C_{M}$ & - & 29 & 9 \\
Maintenance cost, $C_{m}$ & 1,846 & 1,880 & 1,854 \\
Repair cost, $C_{r}$ & 541 & 392 & 504 \\
Revenue loss, $C_{v}$ & 7 & 5 & 6 \\
Societal Economic loss, $C_{e}$ & 1,987 & 1,402 & 1,435 \\
\hline Total LCC & $\mathbf{4 , 3 8 1}$ & $\mathbf{3 , 7 0 8}$ & $\mathbf{3 , 8 0 8}$ \\
Total LCC w/o $C_{\boldsymbol{e}}$ & $\mathbf{2 , 3 9 4}$ & $\mathbf{2 , 3 0 6}$ & $\mathbf{2 , 3 7 3}$ \\
\hline Net benefit & - & $\mathbf{6 7 3}$ & $\mathbf{5 7 3}$ \\
Net benefit w/o $C_{\boldsymbol{e}}$ & - & $\mathbf{8 8}$ & $\mathbf{2 1}$ \\
\hline \hline
\end{tabular}




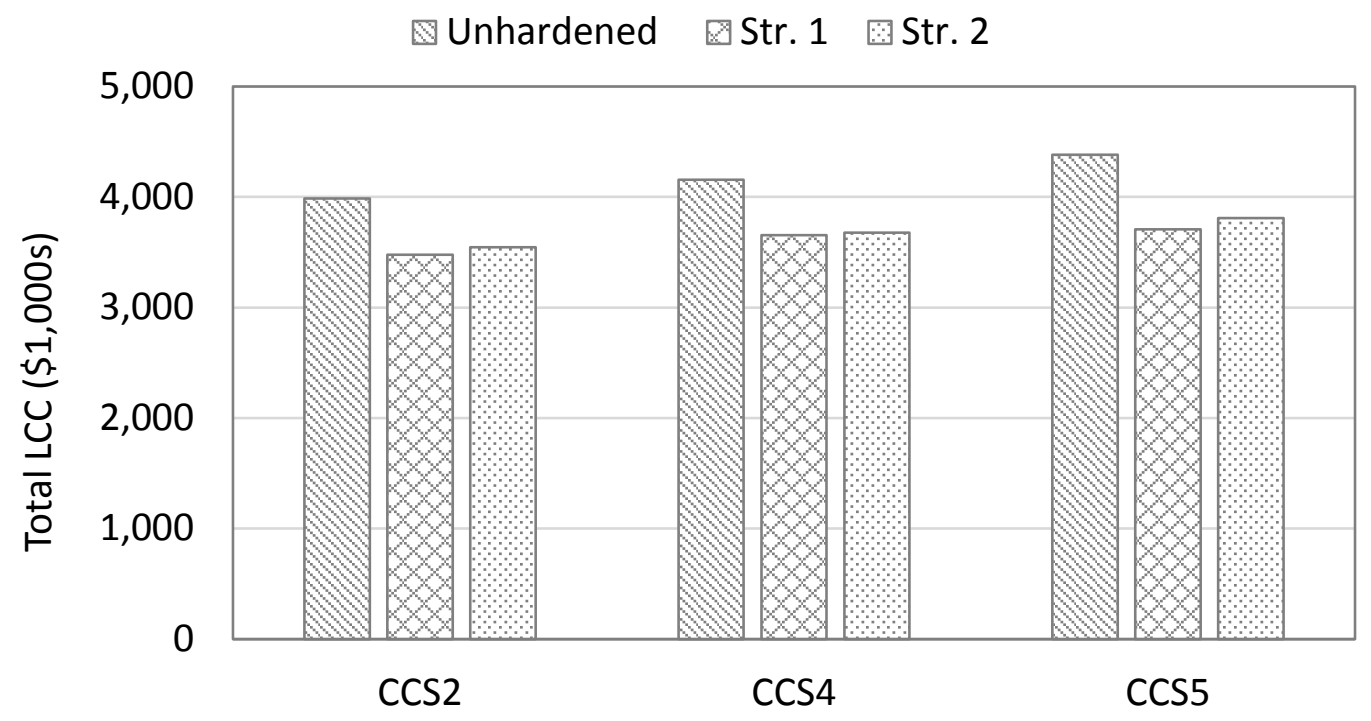

Figure 5.9 Total life cycle cost

From Table 5.6, it can be seen that even for a slight increase in hurricane wind speed (CCS2), both strategies 1 and 2 resulted in a net benefit of nearly $\$ 500,000$. This is mainly because the cost of upgrading to a pole that is one class higher than the required class is very low. For example, class 3 poles cost only $\$ 65$ higher than class 4 poles while class 4 poles cost only $\$ 38$ higher than class 5 poles.

From the results in Table 5.6, Table 5.7, and Table 5.8, the societal economic cost is shown to have a significant impact on the total LCC. In all cases, societal economic losses constitute at least $35 \%$ of the total LCC. The net benefit for all cases dropped considerably if societal economic losses are not considered. For example, in Table 5.6, the net benefit dropped from $\$ 507,000$ to $\$ 53,000$ for strategy 1 if the societal cost is ignored. This is important because considering societal economic losses or otherwise in LCCA depends on the entity carrying out the analysis. Utility companies might not include societal economic losses when making decisions on future investment. However, it might be very important for governments and communities. 
Figure 5.10 shows a plot comparing the net benefit of the adaptation strategies for the three selected climate change scenarios. It can be observed that the net benefit of deploying the adaptation strategies increases with the severity of the climate change scenario especially for strategy 2. For strategy 1, CCS2 and CCS4 showed similar net benefits while the highest net benefit is observed for CCS5 which is the worst climate change scenario.

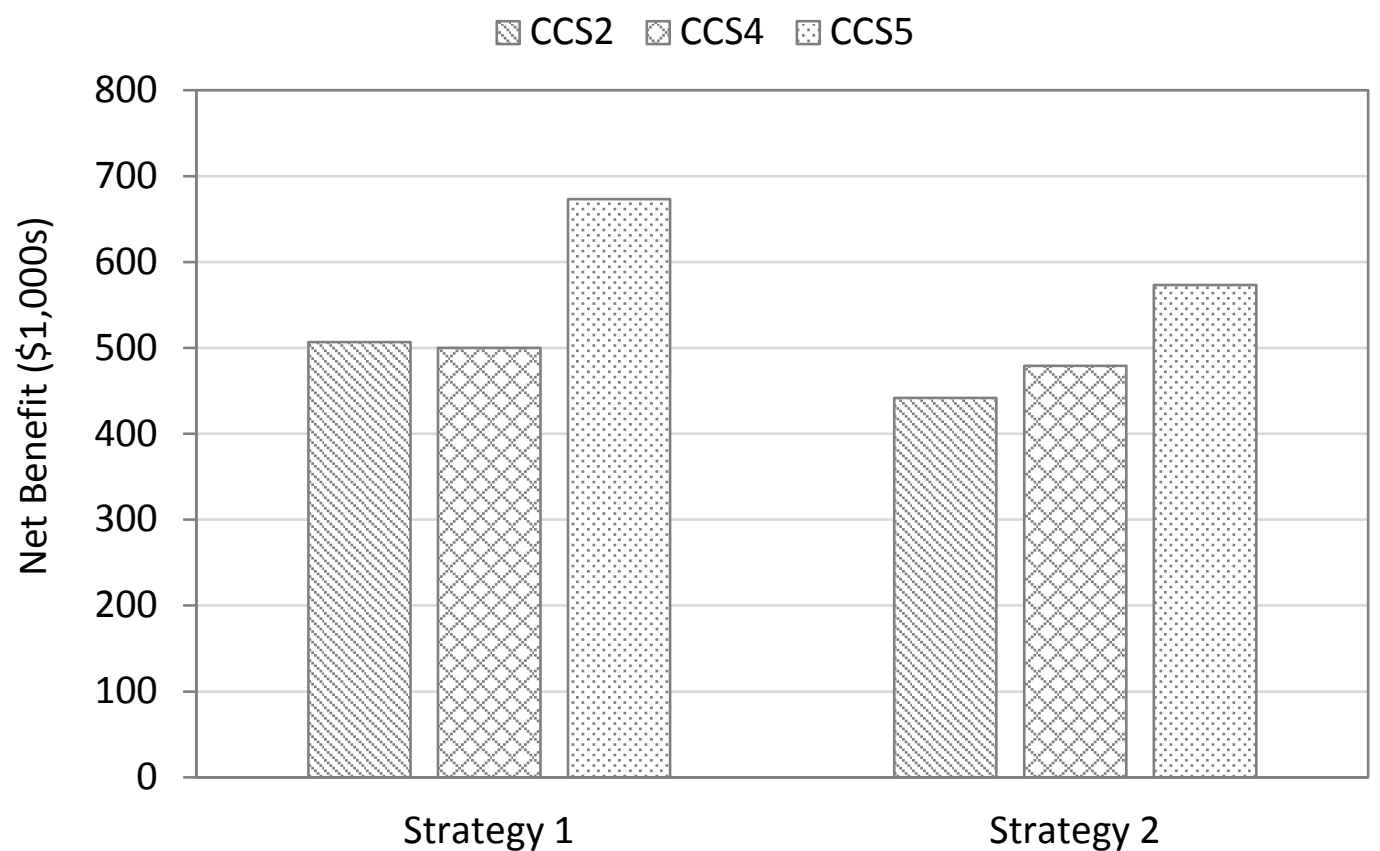

Figure 5.10 Net benefit of adaptation strategies

To account for a reduction in economic activities during disasters, the revenue and economic losses in Table 5.6, Table 5.7, and Table 5.8 are reduced. However, due to lack of literature on the estimate of the reduction in economic activities and power consumption, reduction of 0 to $90 \%$ is considered as shown in Figure 5.11. It can be seen from Figure 5.11 that the net benefit decreases linearly with a reduction in revenue and economic losses. Both adaptation strategies result in positive net benefits for all climate change scenarios even with a reduction of $90 \%$ in revenue and economic losses. 


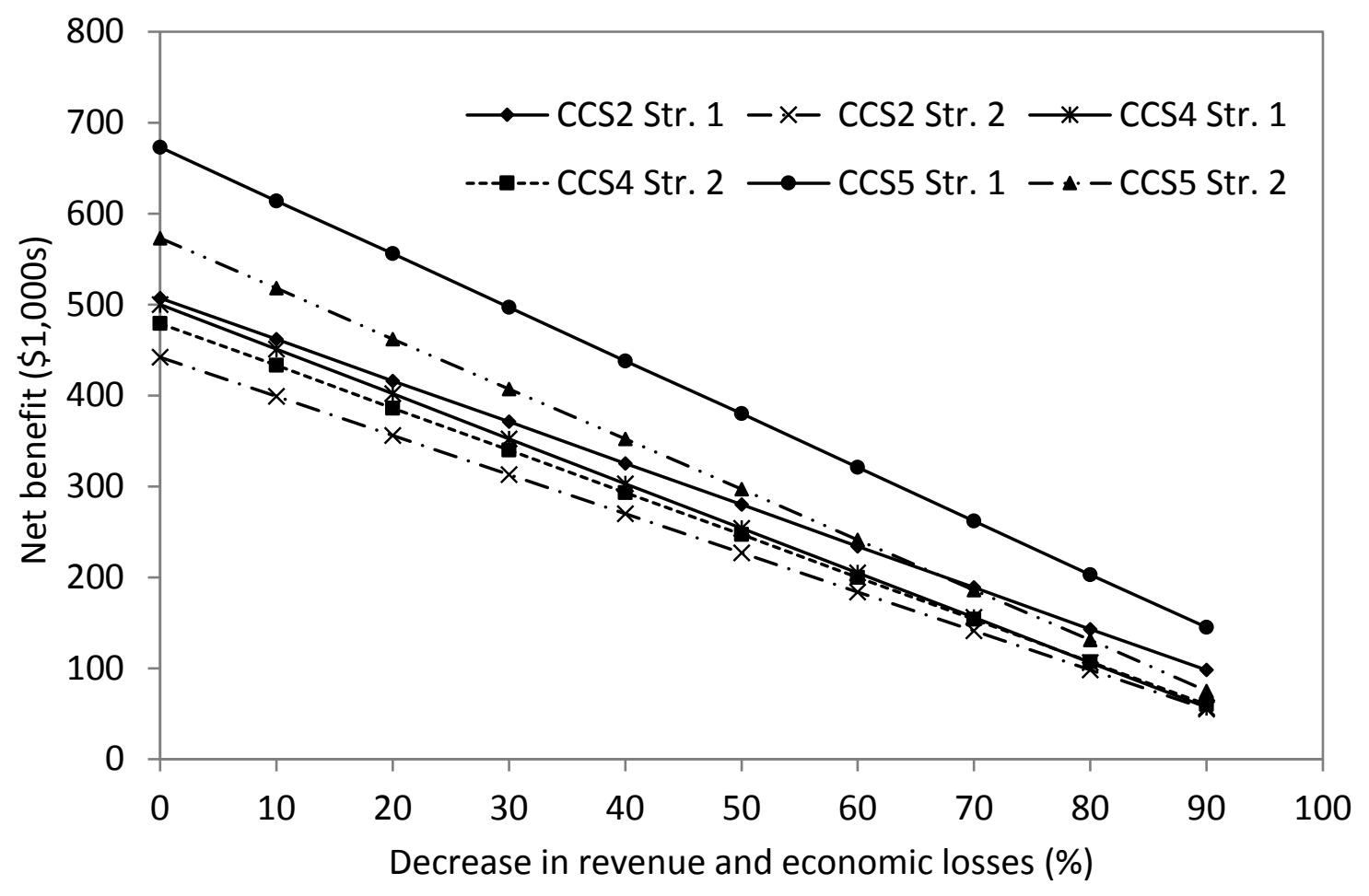

Figure 5.11 Net benefit considering reduction in revenue and economic losses

It should be noted that the results presented in this paper involve a certain degree of uncertainties. For example, there is considerable uncertainty in the prediction of variation of hurricane intensity and frequency. The consideration of six climate change scenarios that cover a range of possible variations is a simple way of taken such uncertainties into account. Uncertainties in hurricane simulation parameters such as approach angle and central pressure difference were accounted for through their corresponding probability distributions as well as by running 200,000 simulations. Uncertainties in the timedependent strength of the poles were considered using the coefficient of variation of the strength of the poles which also varies with time. As the literature on climate change impact on hurricane intensity and frequency as well as the time-dependent strength of poles continues to grow, new findings that can be used to better quantify the uncertainties can be easily incorporated into the proposed framework. 


\subsection{Conclusions}

In this chapter, the potential impact of climate change on distribution systems subjected to hurricanes is evaluated. The effectiveness of various climate change adaptation strategies is also investigated. The framework can be used by utility companies and government agencies for decision making in long-term investment planning and pre-disaster preparedness in power distribution system infrastructure.

The results of the case study show that climate change can have an impact on system reliability and life cycle cost of distribution systems depending on the severity of the potential change. For example, a $35 \%$ increase in frequency and $40 \%$ increase in the intensity of hurricanes will reduce the system reliability by $40 \%$ in 2080 and lead to a $12 \%$ increase in life cycle cost by the end of the century. The results also show that adaptation strategies can be cost-effective in improving system reliability to adapt to the impact of climate change. If the entire system is strengthened at construction stage, the life cycle cost could be reduced by about $15 \%$ by the end of the century for a climate change scenario that resulted in 35\% increase in frequency and $40 \%$ increase in the intensity of hurricanes. The results further demonstrate the importance of considering the cost incurred by society as a whole due to power outages during hurricane events. The framework can be applied directly to an existing system if the age distribution of the poles is known. 


\section{Reconfigurability Enhancement for Distribution Systems Subjected to Hurricanes ${ }^{3}$}

\subsection{Introduction}

Most distribution systems in the U.S. are radial systems (Brown, 2008). In such systems, there is a unique path from the source of power to each component or customer. Consequently, if there is a fault at any point in a line, all customers downstream of the point will lose power. In a region with several cities, independent distribution systems, each with its own substation/substations are used for power supply. In such a case, a potentially costeffective method of storm hardening is rerouting of power from one distribution system to another by constructing additional distribution lines and installing control switches to improve the redundancy of the systems. This will allow customers downstream of failed line to be supplied by a feeder from another substation that is not in the vicinity of a storm. This concept is demonstrated using Figure 6.1 which shows three independent distribution systems.

Constructing additional distribution lines (dotted lines) with normally open (NO) switches to connect feeders from the three independent substations can improve their reliabilities. For example, looking at the hurricane path in Figure 6.1, it is very likely that the upstream of lines 1 and 2, served by substations A and B, will likely fail and cut off power to all customers downstream. In such a case, customers downstream of the affected lines can receive power from feeders 3 and 4 emanating from substation $C$.

\footnotetext{
${ }^{3}$ A version of this chapter has been submitted to Journal of Infrastructure Systems.
} 


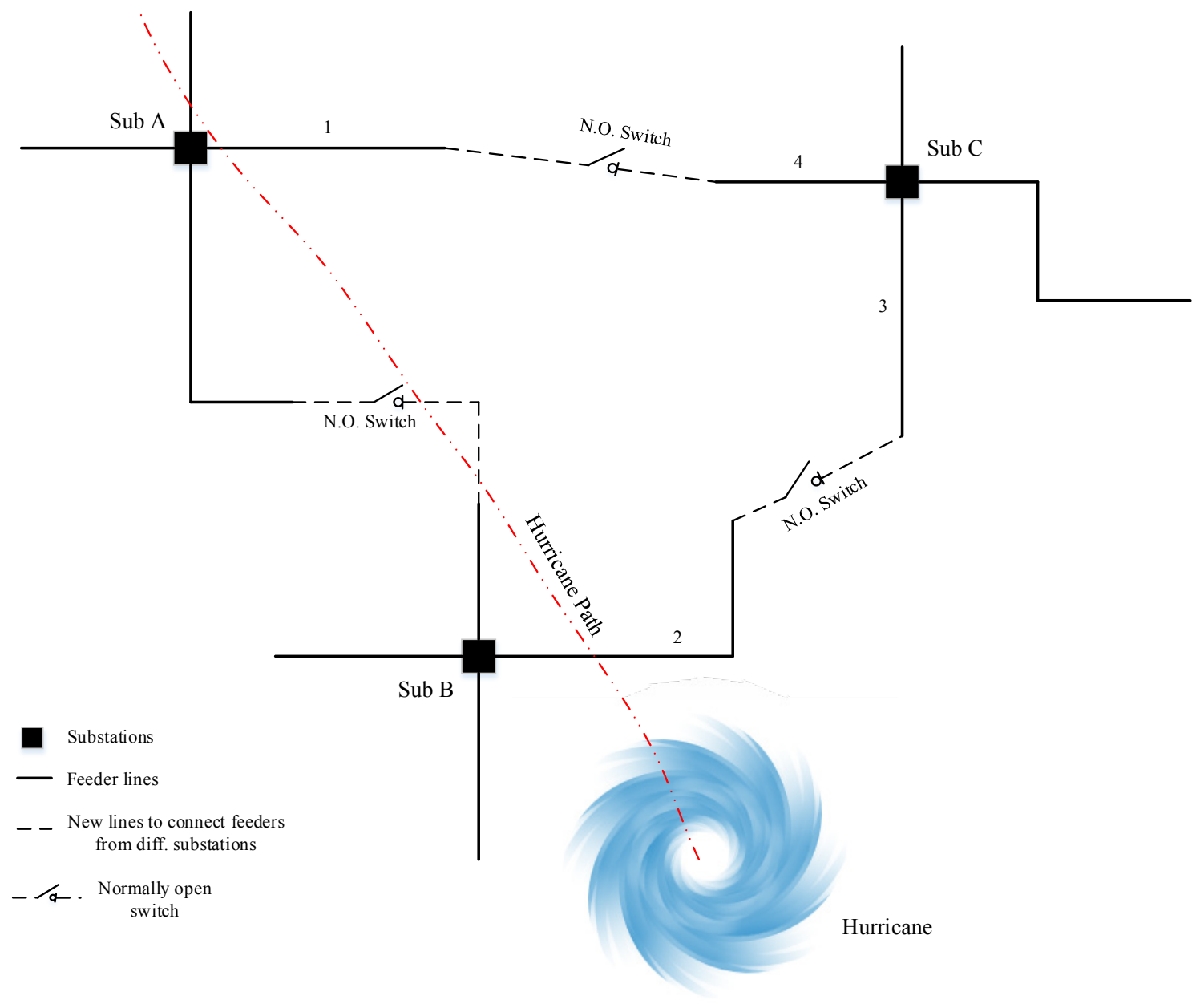

Figure 6.1 Schematic of proposed storm hardening strategy

While rerouting of power within a single distribution system after minor disturbances has been studied over the years (e.g. Viswanadha Raju and Bijwe (2008), Enacheanu et al. (2008), Ramos et al. (2005), Chouhan et al. (2009), Faza et al. (2007)), systematic study of rerouting between independent systems after a catastrophic event such as hurricane that takes into account the topography and the spatial nature of distribution system, as well as hurricane wind has not been carried out. This chapter studies the effectiveness of constructing new distribution lines with Normally Open switches in improving the reliability of radially operated distribution systems subjected to hurricanes. Unlike in 
Chapters 4 and 5 where a single distribution system (one substation and its feeders) was considered, a system of several independent distribution systems is considered here.

\subsection{Power Distribution System Model}

For the purpose of the proposed study, a GIS model of the distribution system of parts of Florida is developed as shown in Figure 6.2. It should be noted that only the 3-phase main feeder lines are shown in Figure 6.2. The region consists of several unconnected networked distribution systems or 'islands'. Each island consists of one or more substation with feeders that are radially operated due to the presence of normally open switches at several locations within the system. The entire study area has 20 substations and 73 feeders. Each feeder is divided into lines (switchable sections) using sectionalizing switches. The distribution system covers an area of approximately 10,000 square miles.

For simplicity, all the laterals lines per feeder mile are lumped as one load point for system reliability evaluation. Based on feeder customer density for the study area reported by the utility company serving the area, the average number of customers is assumed to be 200 per feeder mile. The total length of all the feeder lines in the study area is about 1,080 miles serving approximately 216,800 customers.

Due to lack of data on location of sectionalizing switches in the systems, the following placement points are assumed for the switches in the system:

i. Sectionalizer placement at the beginning of each lateral line

ii. Sectionalizer placement at every branching point of the main feeder lines

iii. Sectionalizer placement at every mile of the main feeder lines 
To enable power reconfiguration in the event of a hurricane, additional lines with tie switches will be constructed to connect any two ends of feeders. This is a simple combinatorial problem where the number of possible combinations is given by $n ! /((n-2) ! 2 !)$. This will result in a very high number of possible combinations as some feeders among the 73 feeders in the area have several ends. To reduce the possible number of combinations, the following constraints are set:

i. New lines will only be constructed to connect feeders from different substations as the aim is to provide additional independent source of power to feeders

ii. Only new lines that connect feeders from different distribution islands will be considered

iii. New lines should not be more 20 miles long

Among the reduced possible number of new lines, the shortest one is chosen for each feeder so as to minimize the cost of construction. In all cases, the radial structure of the system is maintained. Topographical constraints are also considered in the construction of new lines, i.e. new lines are assumed to follow the road network of the study area just like most of the existing distribution lines.

17 new lines are proposed for the study area to demonstrate the framework and are shown in Figure 6.2. Manually-operated Normally Open switches are added to the new lines to maintain the radial nature of the systems. As the aim of this research is to investigate the effectiveness of constructing additional lines with switches to connect feeders between different distribution islands, the optimization of switching operations within each individual island is not considered in the research. 


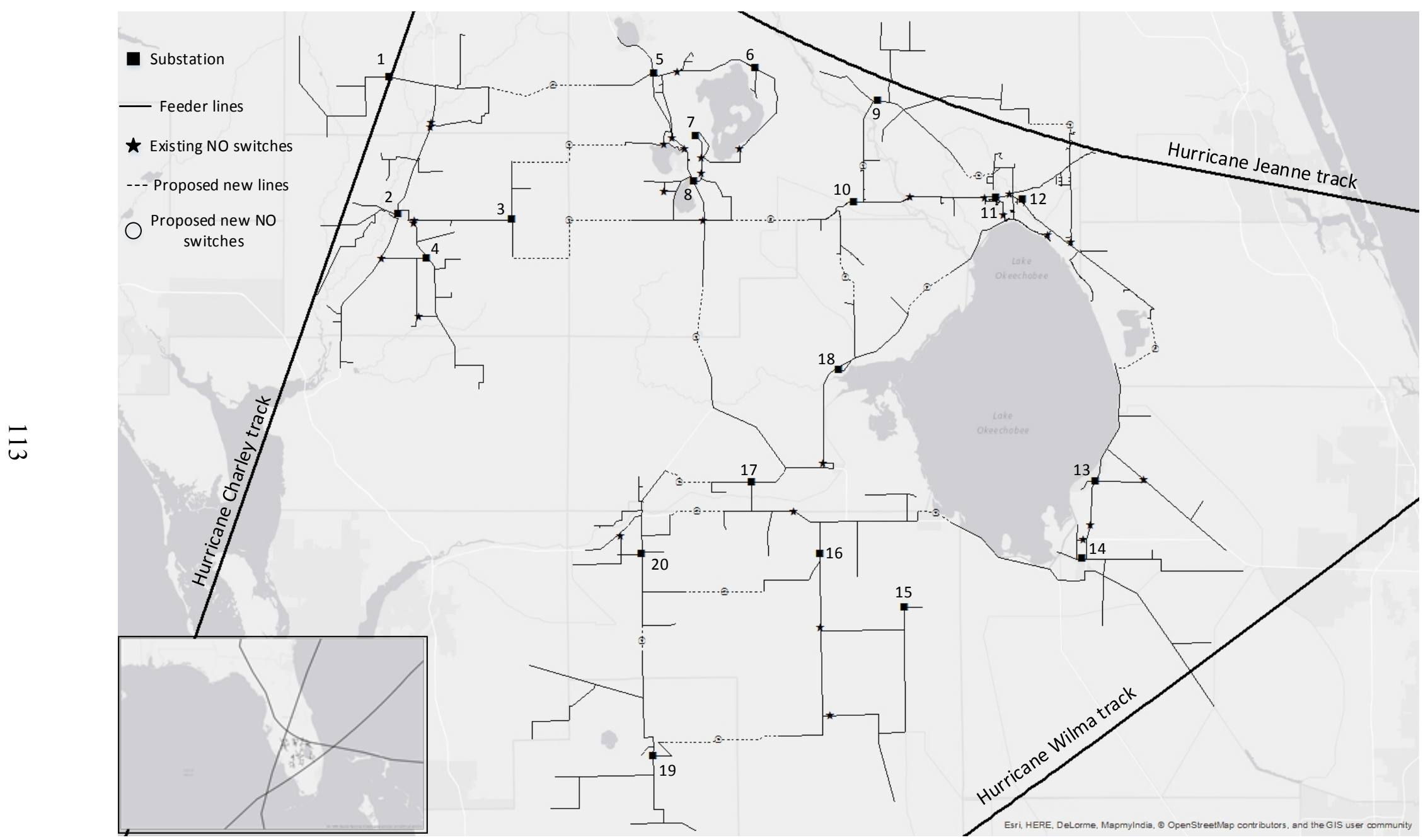

Figure 6.2 Notional power distribution system of parts of Florida (3-phase feeder lines)

[Basemap (C) OpenStreetMap Contributors) 


\subsection{Hurricane Hazard Analysis}

Studying the effectiveness of constructing new distribution lines require consideration of the path and the spatial variation of wind speeds of hurricanes. Hence, for the hurricane hazard analysis, a scenario-based approach is proposed in this framework. This entails selecting historical hurricanes that passed through a selected study area or simulating synthetic hurricanes. Three historical hurricanes are selected for this study. These are Hurricane Jeanne, Hurricane Charley, and Hurricane Wilma. Hurricane Jeanne made landfall on the east coast of Florida on 26 September 2004 as a category 3 hurricane (Lawrence \& Cobb, 2005). Hurricane Charley made landfall on the southwest coast of Florida on 13 August 2004 as a category 4 hurricane (Pasch et al., 2005). Hurricane Wilma made landfall on 24 October 2005 as a category 3 hurricane.

The paths of these hurricanes are shown in Figure 6.2. The variation of gradient wind speed with distance from hurricane eye for the three historical hurricanes as they made landfall is shown in Figure 6.3. The study area is divided into 10 mile x 10-mile grids. For each hurricane, the gradient wind speed at any location at every time instant is calculated and then converted to 3-sec gust wind speed using methodology discussed in Chapter 4. 


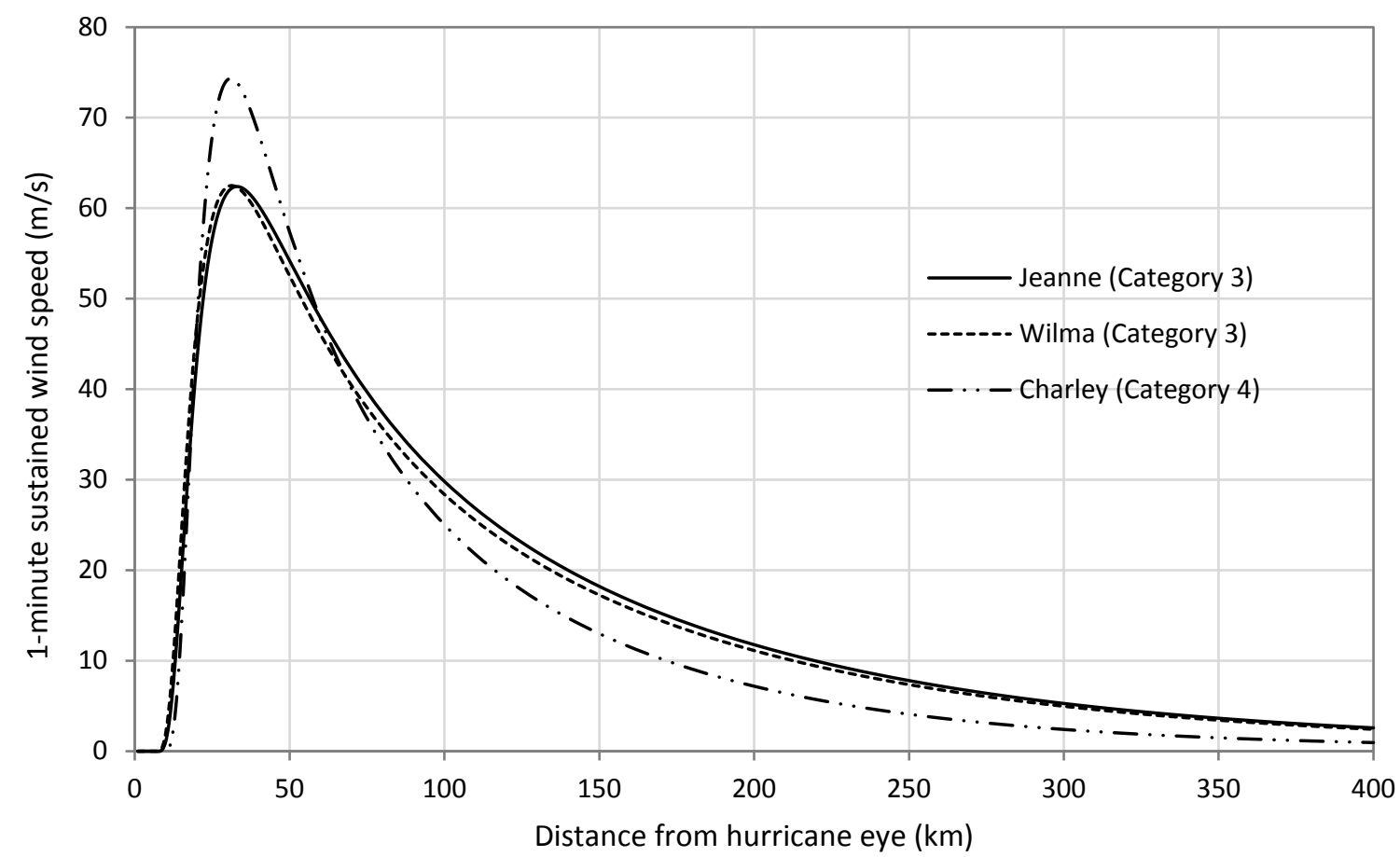

Figure 6.3 Vortex shape of gradient wind speed for the 3 historical hurricanes at landfall

\subsection{Component Vulnerability}

The poles supporting the distribution lines are assumed to be southern pine wood poles as it is the dominant material used in the U.S. (Gustavsen \& Rolfseng, 2000; Wolfe \& Moody, 1997). According to Florida Power \& Light, the company serving the selected study area, class 2 wood poles are used to support main feeder lines as part of their storm hardening plan. It is therefore assumed herein that all the poles supporting the distribution feeder lines are class 2 southern pine wood poles.

A typical distribution pole that is $13.7 \mathrm{~m}$ high is considered. The poles are assumed to support three Aluminum Conductor Steel Reinforced (ACSR) conductor wires with diameters of $18.3 \mathrm{~mm}$ as well as one all-aluminum conductor (AAC) neutral wire with a 
diameter of $11.8 \mathrm{~mm}$. All the poles are assumed to have a span of $46 \mathrm{~m}$ for wind pressure calculations (Short, 2006). It is acknowledged that the span can vary with location and between urban and rural areas. For the purpose of demonstrating the framework, however, the span is assumed to be constant. The fragility curve of the poles is shown in Figure 6.4. Note that in this study, the poles are assumed to be new, i.e. no decay.

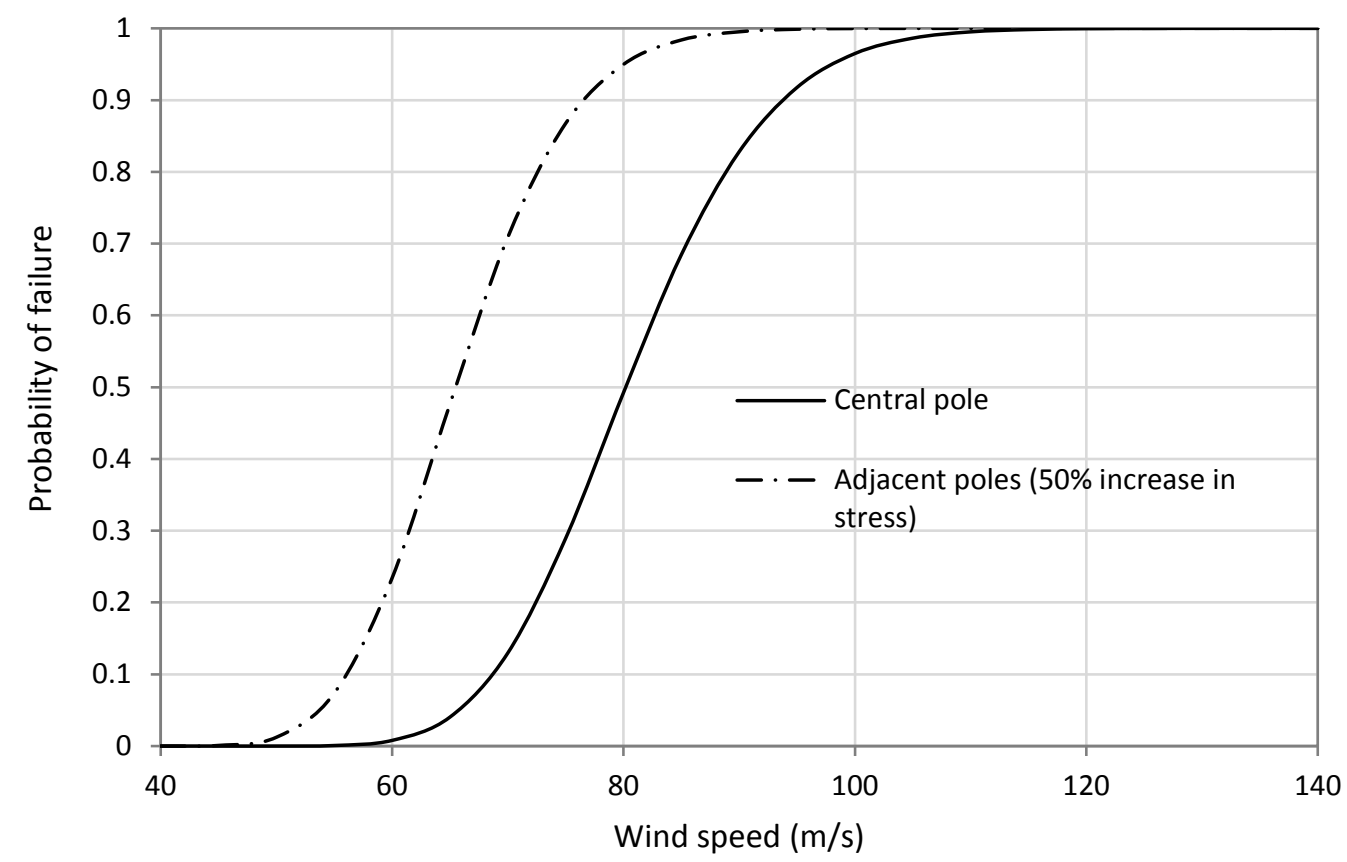

Figure 6.4 Fragility curve of main feeder line poles

\subsection{Cost-Benefit Analysis}

To investigate the cost-effectiveness of constructing additional lines for power rerouting, cost-benefit analysis is performed. The cost is the money spent in constructing the new lines to connect feeders from different substations, while the benefit is the reduction in revenue loss and societal economic losses due to power rerouting. The net benefit is 
calculated by subtracting the cost from the benefit. If the net benefit is positive, then constructing additional lines is considered cost-effective. The various costs considered are discussed below.

i. Mitigation cost: this is the cost of constructing additional distribution lines with NO switches to connect feeders from different substations.

ii. Repair cost: this is the cost of repairing failed distribution lines due to hurricane winds. In this case, it is the cost of replacing failed distribution poles with new poles of the same class. The repair cost is given by Equation (6.1).

$$
C_{r p}=\sum_{i}^{n} P_{f p} \cdot N \cdot C_{r}
$$

where $C_{r p}$ is the repair cost for line $i ; P_{f p}$ is the probability of failure of a pole; $N$ is the number of poles in a line, $C_{r}$ is the unit repair cost of poles; and $n$ is the number of lines in a system.

iii. Revenue loss: this is the cost incurred by the utility company due to the interruption in power supply. The loss due to unmet demand caused by the failure of distribution lines is given by Equation (6.2).

$$
U_{D}=\int_{t=0}^{t_{r}} K_{i}(t) \cdot p(t) d t
$$

where $U_{D}$ is the loss due to unmet demand; $K_{i}(t)$ is the average hourly demand on line $i$ at time $t ; p(t)$ is the unit price of electricity at time $t ; t_{r}$ is the time to restore service to a line. $t_{r}$ depends on the number of failed poles in a line as well as in lines upstream of the line being considered. The total revenue loss is then given by Equation (6.3). 


$$
C_{r v}=\frac{1}{R} \sum_{i}^{n}\left(U_{D} \mid Q_{L i}\right) \cdot \operatorname{Pr}\left(Q_{L i}\right)
$$

where $C_{r v}$ is the total revenue loss for a system; $\left(U_{D} \mid Q_{L i}\right)$ is the loss due to unmet demand given that power is not delivered to line $i ; \operatorname{Pr}\left(Q_{L i}\right)$ is the probability that power is not delivered to line $i ; R$ is the number of repair crew units; and $n$ is the number of lines in a system.

iv. Societal economic losses: this is the direct economic loss to customers due to the interruption in power supply. The economic loss for a line after a hurricane event is given by Equation (6.4).

$$
E_{L}=\int_{t=0}^{t_{r}} L(t) d t
$$

where $L(t)$ is the monetary loss per hour; $t_{r}$ is the time to restore power to a line. The total societal economic loss is then given by Equation (6.5).

$$
C_{E}=\frac{1}{R} \sum_{i}^{n}\left(E_{L} \mid Q_{L i}\right) \cdot \operatorname{Pr}\left(Q_{L i}\right)
$$

where $C_{E}$ is the total societal economic loss for a system; $E_{L}$ is the economic loss for a line; $R$ is the number of repair crew units; and $n$ is the number of lines in a system.

The various costs, restoration times, power consumption, and economic loss for various customer types are given in Table 6.1. Due to lack of data on the number and specific locations of customer types in the study area, it is assumed that 2 customers per feeder mile, or $1 \%$ of all customers per feeder mile, are commercial customers while the rest are residential customers. 
Table 6.1 Cost analysis parameters

\begin{tabular}{l|l|l}
\hline \hline Parameters & Value & Source \\
\hline Cost of constructing new lines (switches included) & $\$ 350,000$ & Gregory et al. (2006) \\
Cost of pole replacement under storm condition & $\$ 4,000 /$ pole & Xu and Brown (2008b) \\
Unit price of electricity & $\$ 0.11 / \mathrm{kWh}$ & Xu and Brown (2008b) \\
Economic loss (residential) & $\$ 2.70 / \mathrm{h}$ & LaCommare and Eto (2006) \\
Economic loss (commercial) & $\$ 886 / \mathrm{h}$ & LaCommare and Eto (2006) \\
Average consumption (residential) & $1.5 \mathrm{~kW} / \mathrm{h}$ & EIA (2013) \\
Average consumption (commercial) & $10.1 \mathrm{~kW} / \mathrm{h}$ & EIA (2013) \\
Number of repair crew units available & 10 & Assumed \\
Restoration time of failed pole & $4 \mathrm{hours}$ & Brown (2009) \\
Discount rate & $4 \%$ & Bastidas-Arteaga \\
\hline \hline
\end{tabular}

\subsection{Results}

\subsubsection{System Reliability Results}

The results of the system reliability analysis are shown in Figure 6.5, Figure 6.6, and Figure 6.7. As mentioned earlier, due to the radial nature of distribution systems, each substation is considered as a separate system. For each line in a system, the maximum wind speed experienced by the line as each hurricane passes through the study area is used in the evaluation of system reliabilities shown in Figure 6.5, Figure 6.6, and Figure 6.7.

It can be observed from Figure 6.5, Figure 6.6, and Figure 6.7 that constructing new lines with NO switches improved the reliabilities of the systems. However, the level of improvement depends on the path of the hurricane, number of new lines connected to a system, and length of the new lines. For example, the reliability of system 9 only improves 
by about $30 \%$ when hurricane Jeanne passes through even though there are 3 new lines supplying the system from 3 different substations. This is because both the alternative sources of energy, as well as the new lines, are in the path of the hurricane. Considering system 10, however, which also has 3 new lines connected to it, its reliability improved by about $79 \%$ because two of the three new lines, as well as the substations feeding them, are further away from the path of hurricane Jeanne. The length of the new lines affects the level of improvement in system reliability because the longer the lines, the higher the probability of failure during hurricanes. In this case, most of the new proposed lines are more than 10 miles long. This drastically limits the effect these lines will have in improving system reliability.

Figure 6.7 shows that hurricane Charley mostly affected systems $1-5$ as can be inferred from the path of the hurricane in Figure 6.2. Among these 5 systems, only systems 1, 3, and 5 have new additional lines. The reliability of system 3 increased from about $1 \%$ to $3 \%$ due to the additional lines. 


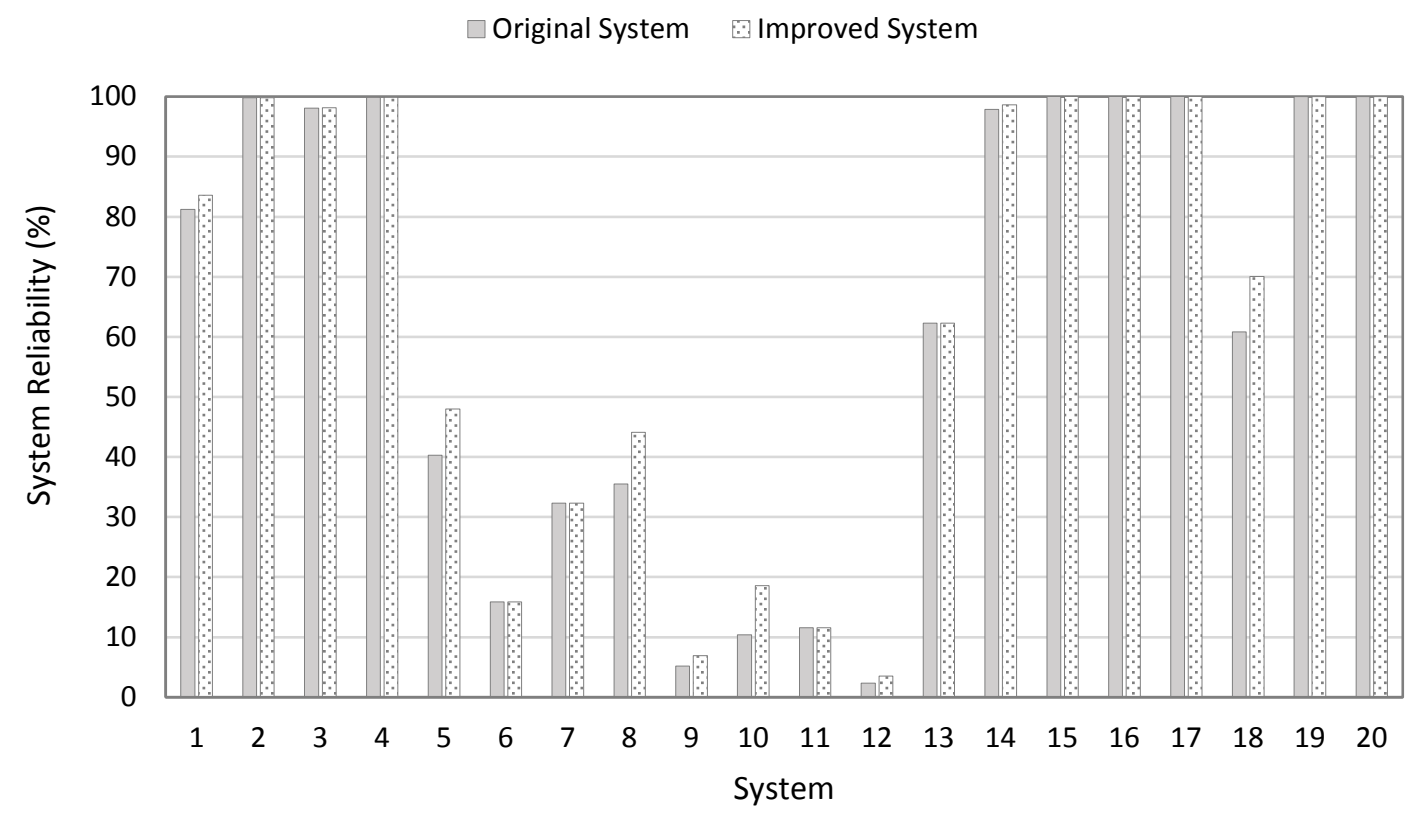

Figure 6.5 System reliability results for Hurricane Jeanne

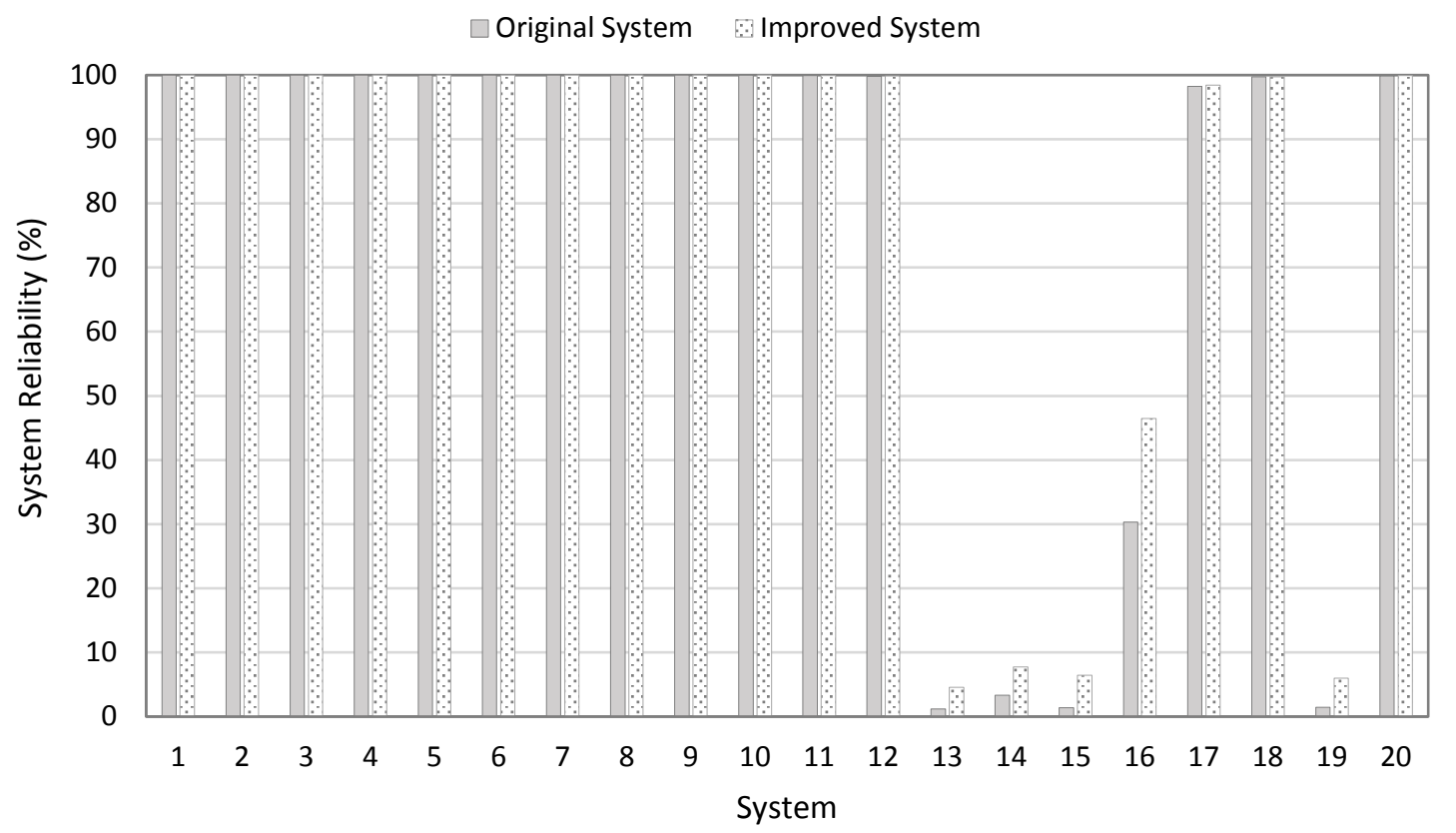

Figure 6.6 System reliability results for Hurricane Wilma 


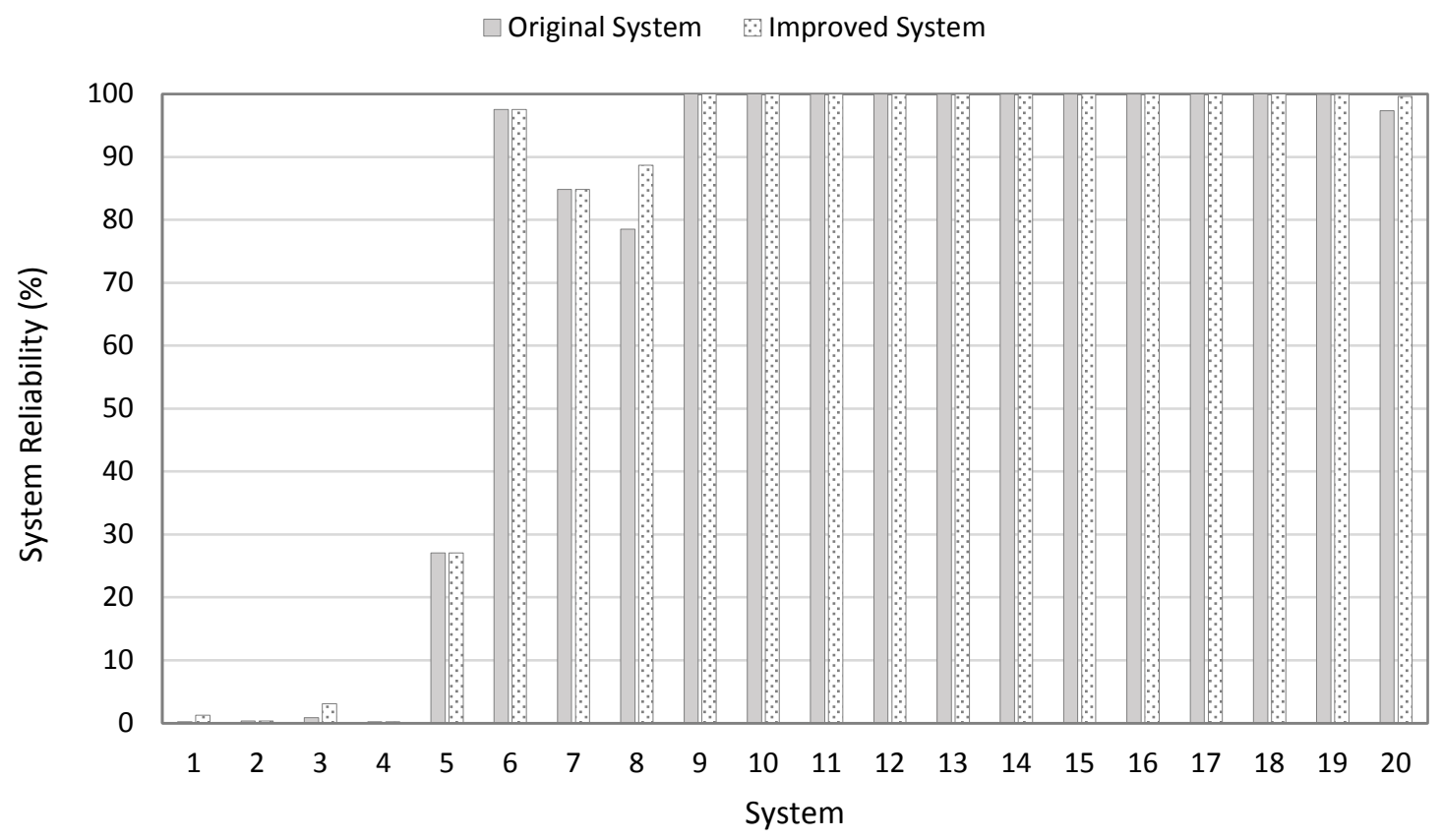

Figure 6.7 System reliability results for Hurricane Charley

\subsubsection{Cost Analysis Results}

The results of the cost analysis are shown in Table 6.2. It can be seen that in general, constructing the new distribution lines for the system considered is not cost-effective. This is due to the high cost of constructing the new lines which, in this case, is over $\$ 50$ million as seen in Table 6.2. This is mainly due to the long distance between the ends of the feeders connected by the new lines. Additionally, some poles in the new lines will fail especially for a new line in the path of the hurricane. This leads to increase in repair cost for the modified system as compared to the original system. The repair cost increased by about $17 \%, 11 \%$, and $11 \%$ for the modified system after Hurricanes Jeanne, Wilma, and Charley, respectively.

The addition of new lines did not significantly reduce revenue loss and societal economic losses. Revenue loss decreased by about $2.6 \%, 1.5 \%$, and $0.4 \%$ for Hurricanes Jeanne, 
Wilma, and Charley, respectively. Societal economic losses decreased by $3.7 \%, 1.4 \%$, and $0.2 \%$, for Hurricanes Jeanne, Wilma, and Charley, respectively. In fact, the increase in repair cost is greater than benefit from the decrease in revenue loss and societal economic losses combined. This implies that in this case, repair cost should be the main concern for utility companies.

Table 6.2 Cost analysis results $(\$ 1,000 \mathrm{~s})$

\begin{tabular}{lllllll}
\hline \hline & \multicolumn{2}{l}{ Hurricane Jeanne } & \multicolumn{2}{l}{ Hurricane Wilma } & \multicolumn{2}{l}{ Hurricane Charley } \\
\cline { 2 - 7 } Cost Category & Original & Modified & Original & Modified & Original & Modified \\
& system & system & system & system & system & system \\
\hline Mitigation & - & 50,190 & - & 50,190 & - & 50,190 \\
Repair & 1,471 & 1,722 & 2,059 & 2,289 & 3,004 & 3,329 \\
Revenue loss & 76 & 74 & 136 & 134 & 232 & 231 \\
Societal economic loss & 5,049 & 4,864 & 8,972 & 8,849 & 15,317 & 15,291 \\
& & & & & & \\
Net benefit (NB) & - & $\mathbf{- 5 0 , 2 5 4}$ & - & $\mathbf{- 5 0 , 2 9 5}$ & - & $\mathbf{- 5 0 , 4 8 9}$ \\
NB w/o societal cost & - & $\mathbf{- 5 0 , 4 3 8}$ & - & $\mathbf{- 5 0 , 4 1 8}$ & - & $\mathbf{- 5 0 , 5 1 5}$ \\
\hline \hline
\end{tabular}

\subsection{Conclusions}

This chapter studies system reliability improvement due to power reconfiguration through the construction of additional lines with normally open (NO) switches to connect systems supplied by different power sources. Cost analysis to study the cost-effectiveness of the proposed risk mitigation strategy is also performed. The case study used indicate that constructing new lines to connect feeders from different substations can improve the reliability of the systems. However, the level of improvement depends largely on hurricane path, the number of new lines constructed as well as the length of the new lines. 
In the case study considered, construction of additional lines is not cost-effective mainly due to the high cost of constructing the lines. It can be concluded that the cost-effectiveness of such strategy will largely depend on the length of the new lines that need to be constructed. While the mitigation strategy might not be attractive economically, the improvement in system reliability can improve customer satisfaction and help maintain power supply to critical facilities during natural disasters. Additionally, increase in repair cost due to the addition of new distribution lines can outweigh savings from both revenue loss and societal economic losses. Consequently, mitigation strategies that lower the repair cost might be more attractive, economically, for utility companies. This includes using stronger poles for the lines or shortening the spans of the lines.

In this study, a topological-based method of evaluating system reliability was used. This method only takes into account the topological constraint in distribution system reconfiguration and does not incorporate the engineering or physical aspects of the system which will require performing complex power flow analysis. To account for electrical constraints such as voltage drop and equipment overload, a complete power flow analysis based on the physical and engineering details of the system is recommended for future research. 


\section{Multi-Hazard Risk Assessment Framework ${ }^{4}$}

\subsection{Introduction}

Natural hazards can be concurrent/non-concurrent and dependent/independent. For example, high winds, waves, and storm surge during hurricanes are dependent and concurrent hazards. Earthquakes can cause tsunamis, landslides, and fires which make them dependent hazards. Seismic and hurricane hazards can be described as independent and non-concurrent hazards. Regardless, within the life span of infrastructure systems located in regions vulnerable to both hazards, there is a possibility of such infrastructure being subjected to such independent hazards that are different in nature. Therefore, effective mitigation of risks to infrastructure due to natural hazards requires understanding, evaluation, and interaction of (a) all hazards that can cause significant threats and, (b) the vulnerability of infrastructure subjected to the hazards. Consequently, the United Nations (UN) in its Johannesburg Plan called for increased effort in integrated, multi-hazard risk assessment as part of a comprehensive disaster management plan (UN, 2002).

Hazard events differ in nature, intensity, return periods, and magnitude measurement method. Therefore, the first challenge of multi-hazard risk analysis is comparability of hazardous events (Carpignano et al., 2009; Li et al., 2012; Marzocchi et al., 2012). Hazards with different probabilities of occurrence, such as earthquakes and hurricanes, are difficult to compare. For example, a low probability/high consequence earthquake can cause as much damage as recurrent high probability/low consequence hurricanes. The second difficulty in multi-hazard risk analysis is the comparison of vulnerabilities of exposed elements (Carpignano et al., 2009). Different hazards can affect different elements in a region or different components of a system. For example, substations and transmission lines can be more vulnerable to different hazards and the parameters used to measure their vulnerabilities are not the same.

\footnotetext{
${ }^{4}$ A version of this chapter has been submitted to ASCE Journal of Structural Engineering.
} 
Multi-hazard risk assessment pertaining dependent concurrent hazards is approached in two ways. One way is to analyze each single hazard independently and then calculate multihazard risk by weighted summation of single-hazard risk results or indices (Bell \& Glade, 2004; Mosquera-Machado \& Dilley, 2009). The other approach is a truly integrated analysis of hazards, exposure, and vulnerability through joint probability distribution of hazards, multi-dimensional vulnerability, and integration of vulnerability surface with joint hazard distribution (Ming et al., 2015). Multi-hazard risk assessment of independent nonconcurrent hazards is usually carried out through comparative approach using a common index. This is feasible because risk is not measured in hazard-specific units but in damage or loss-specific units such as damage to properties or disruption to economic activities (Kappes et al., 2012).

While development of multi-hazard risk analysis framework for buildings and bridges have been ongoing in recent years, risk analysis of spatially-distributed civil infrastructure systems such as electric power and water systems have so far been limited to mostly singlehazard considerations (e.g. Adachi and Ellingwood (2010), Winkler et al. (2010), Song and Ok (2010), Duenas-Osorio and Hernandez-Fajardo (2008), Kim and Kang (2013), Ryan et al. (2014a)). As these systems usually cover large areas and can be subjected to multiple hazards within their lifetime, there is a need to develop a framework to study the impact of multiple hazards on such systems. This is essential for pre-disaster decision making regarding mitigation strategies as certain mitigation strategies for one hazard might be ineffective or even increase the risk for other hazards.

Ouyang et al. (2012) conducted a multi-hazard study of electric power systems subjected to concurrent hazards as part of a multi-stage resilience framework. The concurrent hazards considered are hurricane hazard and random hazards (equipment failure, trees, animals, human errors) whose co-occurrence was modeled by a Poisson process. Multi-hazard effect of concurrent hurricane and random hazards was also studied in the context of cascading failure of interdependent infrastructure systems by Ouyang and Dueñas-Osorio (2011). The 
hazards considered in the above studies differ greatly in terms of frequency and consequence. Whereas hurricanes usually cause large disruptions and widespread damage to infrastructure systems, damage due to random hazards is usually localized and relatively short in duration.

This chapter presents a framework for multi-hazard risk assessment of electric power systems under seismic and hurricane wind hazards. Two multi-hazard risk assessment methods are also presented. The first method is a comparative approach using proposed risk curves due to multi-hazard, while the second method is a cumulative approach based on the annual probability of system failure. The proposed multi-hazard risk assessment models can be used to prioritize investment in mitigation strategies by ranking hazards based on the level of risk they pose in the short- and long-term.

Figure 7.1 shows a flowchart of the proposed framework. In this chapter, the risk assessment part of the framework (items 1 to 4 of the left side of Figure 7.1) is discussed. Multi-hazard risk mitigation strategies and their cost-effectiveness are discussed in Chapter 8. The framework is demonstrated using a notional power system assumed to be located in Charleston, SC, New York, NY, and Seattle, WA. The proposed framework considers the fragilities of transmission lines and substations. The proposed framework can also be extended to carry out multi-hazard risk assessment of distribution systems when data for fragilities of distribution components (lines and poles) subjected to earthquakes, hurricane wind, falling trees, and flying debris is available. 


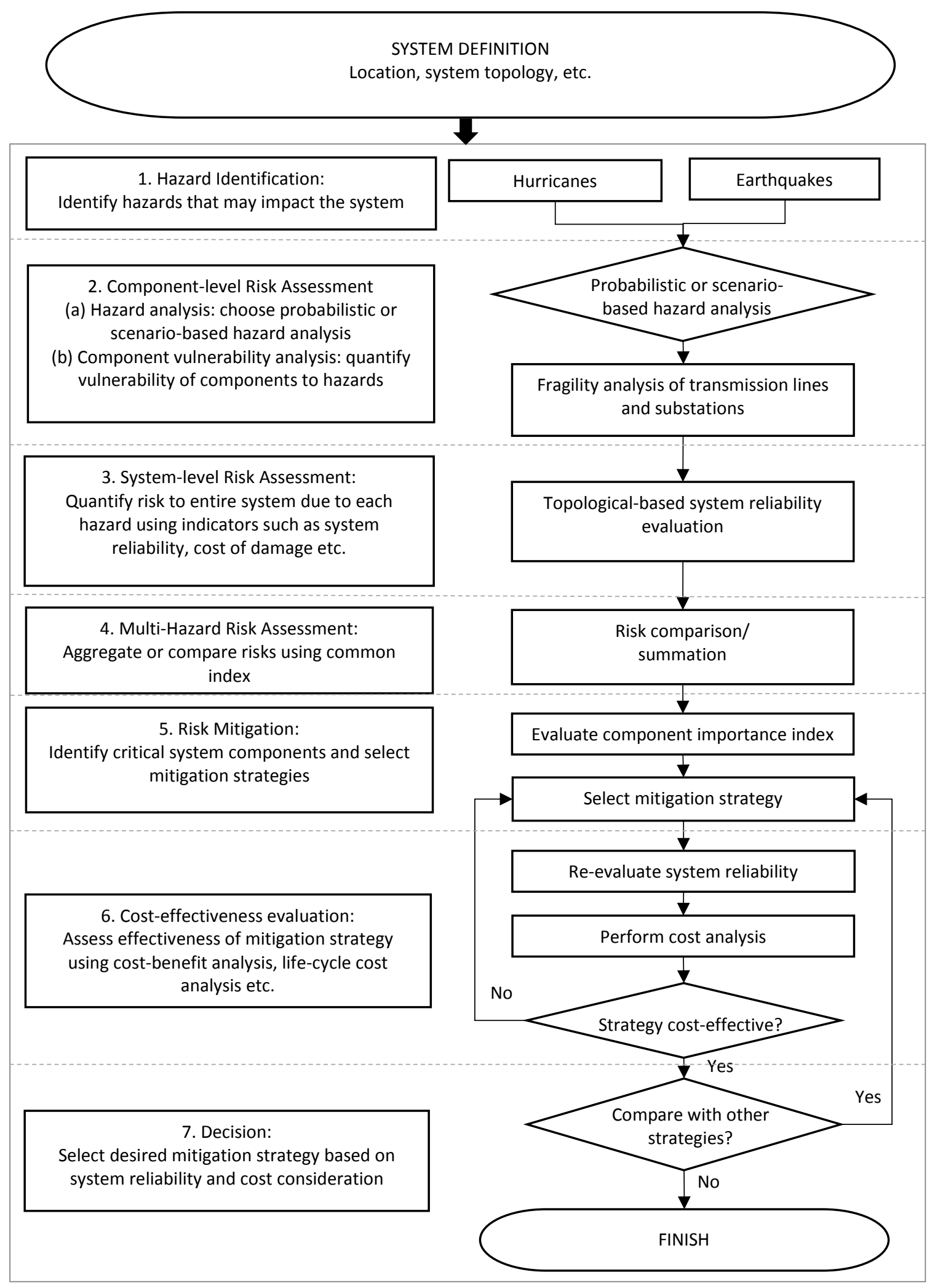

Figure 7.1 Flowchart of proposed multi-hazard risk assessment framework 


\subsection{Electric Power System}

The electric power system adopted to demonstrate the proposed framework is shown in Figure 7.2 and is based on the electric power system of Shelby County, Tennessee modified from Shinozuka et al. (1998). It is assumed herein that the system is located in three cities, namely Charleston, New York, and Seattle. The power system is superimposed on the map of the three locations using the georeferencing tool in ArcGIS. This allows the coordinates of each substation or any point within the system to be determined. 


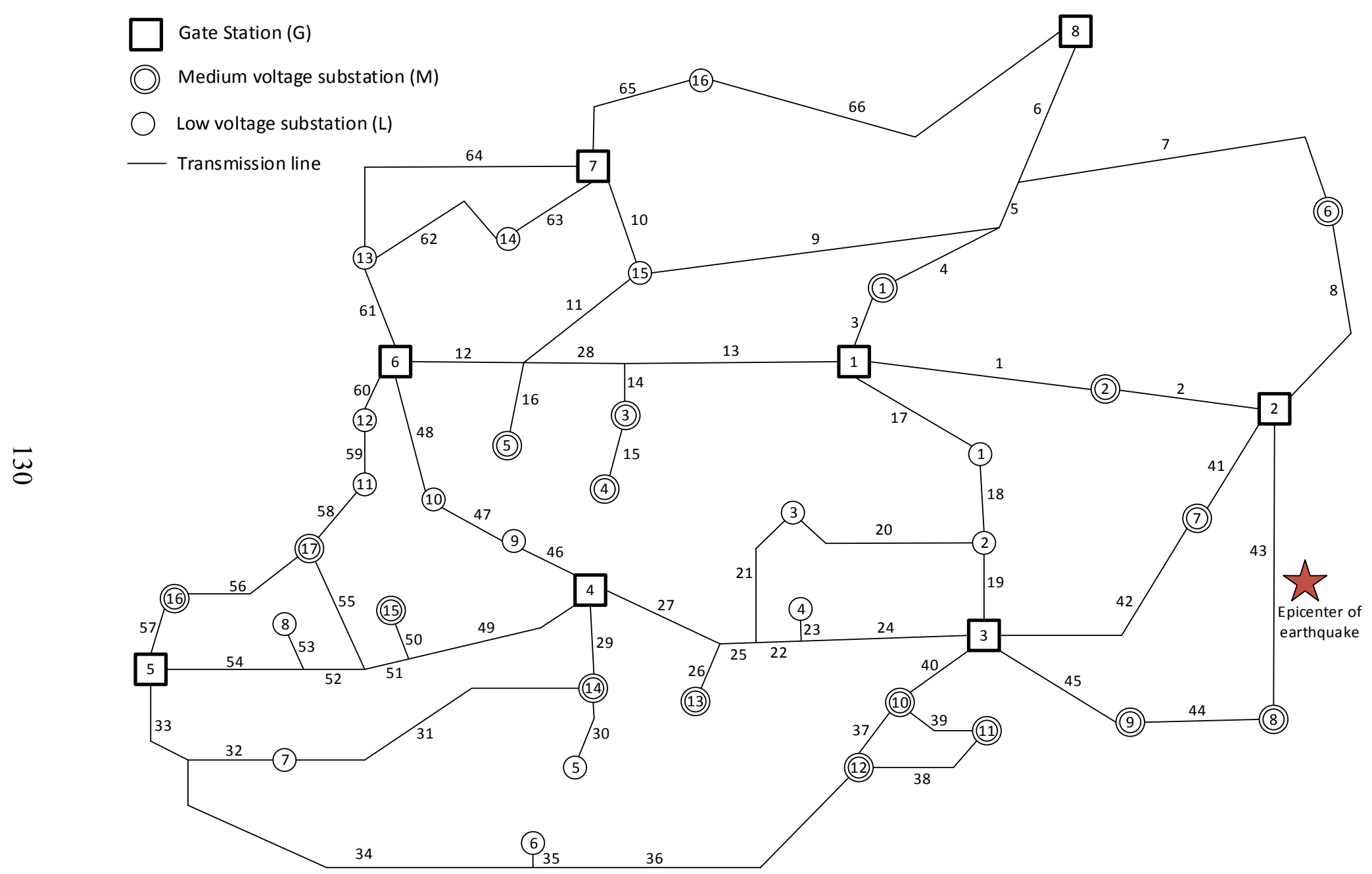

Figure 7.2 Notional electric power system 
The power system consists of high voltage gate stations, medium voltage substations, and low voltage substations. The gate stations are assumed to be the source nodes or supply stations in this case since the system doesn't have generating plants of its own. The medium and low voltage substations are the demand nodes which are distribution substations that directly serve customers. Power flow through the network is modeled so that edges connected to supply nodes are unidirectional while all other edges are bidirectional except those supplying terminal substations such as L5 and M5 in Figure 7.2. To estimate the number of transmission structures in each line, the span is assumed to be $800 \mathrm{ft}$. (Philipson \& Willis, 2006; Davidson et al., 2003).

Number of customers is adopted for use in evaluating system reliability. The number of customers served by the system in its original location in Shelby county, Tennessee is about 400,000 (Shelby-County, 2015). Based on this information and for the purpose of demonstrating the proposed framework, all the low voltage substations are equally assumed to serve 10,000 customers each while the medium voltage substations are assumed to serve 14,000 customers each. The total number of customers served by the system is therefore 398,000. Detailed information on the power system is given in Table 7.1. 
Table 7.1 Notional power system information

\begin{tabular}{ll}
\hline \hline Description & \\
\hline Number of high voltage gate stations (supply nodes) & 8 \\
Number of medium voltage distribution substations (demand nodes) & 17 \\
Number of low voltage distribution substations (demand nodes) & 16 \\
Approximate area covered by system & $2,590 \mathrm{~km}^{2}$ \\
Total number of customers served by system (based on actual numbers & 398,000 \\
from Shelby-County (2015)) & 10,000 \\
Number of customers served by low voltage substations & 14,000 \\
Number of customers served by medium voltage substations & 66 \\
Number of transmission lines & $244 \mathrm{~m}(800 \mathrm{ft})$. \\
Span of transmission line support structures & 1,715 \\
Total number of transmission structures & $33^{\circ} \mathrm{N}, 80.2^{\circ} \mathrm{W}$ \\
Location of G1 in Charleston, SC & $40.76^{\circ} \mathrm{N}, 73.47^{\circ} \mathrm{W}$ \\
Location of G1 in New York, NY & $47.6^{\circ} \mathrm{N}, 122.3^{\circ} \mathrm{W}$ \\
Location of G1 in Seattle, WA & \\
\hline
\end{tabular}

\subsection{Hazard Analysis}

Hazard analysis for structures and infrastructure systems can be carried out in two ways, namely probabilistic analysis and scenario-based analysis (Adachi \& Ellingwood, 2010; $\mathrm{Li}, 2012$ ). Probabilistic analysis considers the aggregated effect of all possible hazard levels. In a probabilistic analysis, hazard levels are weighted by their respective probability of occurrence. In a scenario-based approach, the effect of a specific hazard level is considered (e.g. 200-year return period hurricane or a magnitude 6.5 earthquake).

In the context of multi-hazard analysis where risks due to different hazards are compared using an index, adopting a scenario-based approach such as comparing worst case scenarios of various hazards can be biased (Li \& Ellingwood, 2009). In such a case, all possible 
intensities of hazards should be considered which makes probabilistic hazard analysis more suitable for multi-hazard risk assessment. However, application of probabilistic seismic hazard analysis to spatially distributed infrastructure systems has been shown to be limited by Adachi and Ellingwood (2010). This is because the spatial variation of intensity for a severe earthquake is lost in the aggregation process of probabilistic analysis. The probabilistic approach, however, allows risks to be annualized which is essential in decision making regarding long-term investment in mitigation strategies. This approach also provides a way for risk comparison due to different competing hazards. In this chapter, both probabilistic and scenario-based hazard analysis are considered and discussed. The limitation of both approaches can be overcome by adopting a probabilistically weighted deterministic hazard scenarios approach. This will be discussed in Chapter 8 .

\subsubsection{Seismic Hazard Analysis}

To perform probabilistic seismic hazard analysis (PSHA), seismic-hazard source and attenuation models are used. Seismic-hazard source model describes the location, magnitude and occurrence time of an earthquake while attenuation models describe the decay of seismic intensity from source to a particular site. The end result of PSHA is a seismic hazard curve that gives the annual rate of exceedance of a ground motion intensity measure (e.g., peak ground acceleration or spectral acceleration). The most extensively used hazard curves are developed by the U.S. Geological Survey (USGS) based on the mainshock records of the U.S. (USGS, 2015a; Petersen et al., 2014). The annual rate of exceedance of seismic intensity measure, $I M$, is often modeled by a power law expression such as the one given by Equation (7.1) (Cornell et al., 2002; Sewell et al., 1996; Kameshwar \& Padgett, 2014).

$$
v(I M)=k_{0}(I M)^{-k}
$$


where $v(I M)$ is the annual probability of exceeding intensity measure, $I M$; and $k_{0}$ and $k$ are empirical constants. The power law in Equation (7.1) is linear on a log-log space. Bradley et al. (2007) demonstrated that the above power law overestimates the hazard within the low and high-intensity regions of the hazard curve and underestimates the hazard between the design basis earthquake (DBE) and maximum considered earthquake (MCE) intensity levels (see Figure 7.3). To remedy such anomaly, Bradley et al. (2007) proposed a hyperbolic function in a log-log space as given by Equation (7.2).

$$
\hat{v}=a \cdot \exp \left[b\left\{\ln \left(\frac{P G A}{c}\right)\right\}^{-1}\right]
$$

where $\hat{v}$ is the annual probability of exceeding a certain peak ground acceleration; $a, b$ and $c$ are constants determined by fitting the above equation over a hazard curve such as the one obtained from USGS (2015a).

Figure 7.3 shows the hazard curve plotted using data from USGS (2015a) for a location in a coastal area of South Carolina. The power model in Equation (7.1) is fitted to the USGS hazard curve using the method proposed by Jalayer (2003) while the hyperbolic model in Equation (7.2) is fitted using non-linear least square regression analysis. The constants $k_{0}$ and $k$ in Equation (7.1) are found to be 0.000192 and 1.072, respectively. The constants $a$, $b$, and $c$ in Equation (7.2) are found to be $0.33,30.02$, and 42.36, respectively. It can be seen from Figure 7.3 that the hyperbolic function is more suited to the curve for the entire range of seismic intensities. The hyperbolic function is therefore adopted for use in this research. The steps for the probabilistic seismic hazard analysis are shown in Figure 7.4. 


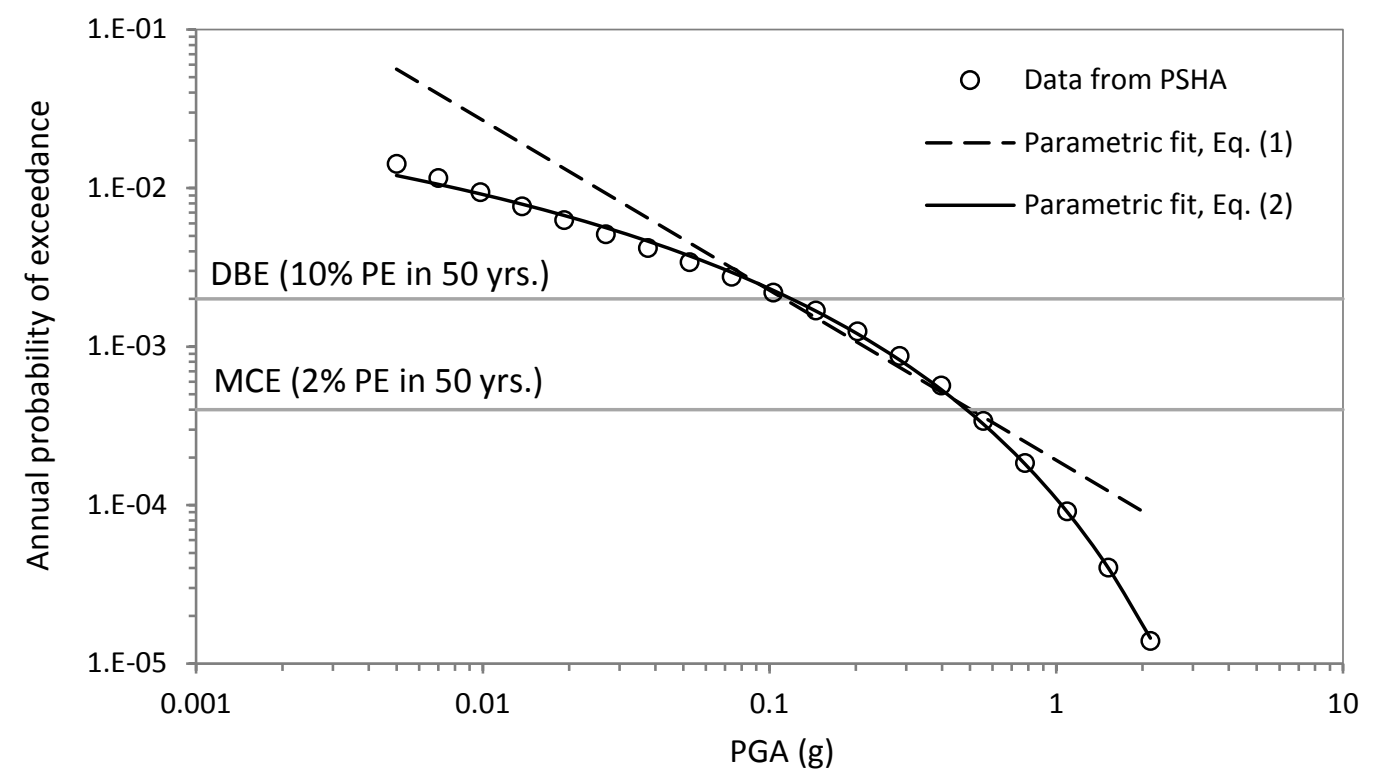

Figure 7.3 Seismic hazard curve for Charleston, SC (32.8oN 79.9oW)

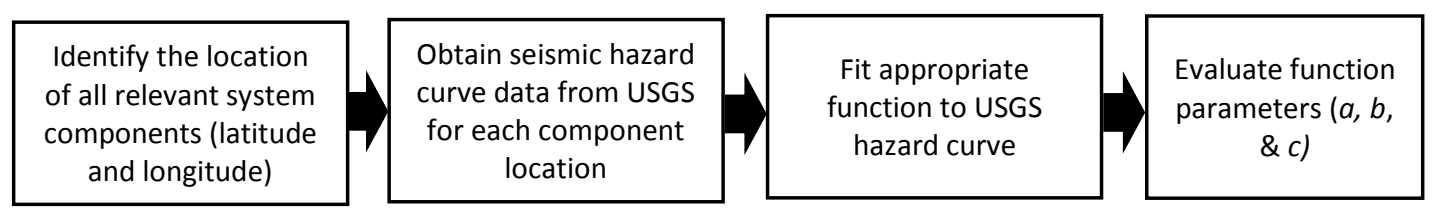

Figure 7.4 Probabilistic seismic hazard analysis

In a scenario-based approach, the seismic intensity at various locations away from the epicenter of a given earthquake is determined using available attenuation models. Toro et al. (1997) developed a stochastic attenuation relationship from extensive analysis of ground motion data for central and eastern North America. The result of the study can be directly applied to hard rock and can be applied to soil sites by using amplification factors. The proposed attenuation equation is:

$$
\begin{aligned}
\ln (P G A)=C_{1} & +C_{2}(M-6)+C_{3}(M-6)^{2}-C_{4} \ln R_{M} \\
& -\left(C_{5}-C_{4}\right) \max \left[\ln \left(\frac{R_{M}}{100}\right), 0\right]-C_{6} R_{M}+\varepsilon_{e}+\varepsilon_{a}
\end{aligned}
$$




$$
R_{M}=\sqrt{R_{j b}^{2}+C_{7}^{2}}
$$

where PGA is the peak ground acceleration in units of g; $C_{1}$ through $C_{7}$ are constants; $M$ is either moment magnitude or body wave magnitude; $R_{j b}$ is the closest horizontal distance to the earthquake rupture in $\mathrm{km}$; and $\varepsilon_{e}$ and $\varepsilon_{a}$ are measures of epistemic and aleatory uncertainties respectively and depend on magnitude and distance. This model is adopted for use in this research.

The 1886 Charleston earthquake which is the strongest earthquake on record to hit South Carolina is selected for demonstrating the framework. The earthquake occurred on August 31,1886 , and had a magnitude of 7.3 and epicenter at $32.9^{\circ} \mathrm{N}$ and $80^{\circ} \mathrm{W}$ shown in Figure 7.2 (USGS, 2015b). The attenuation model given by Equation (7.3) and soil amplification factors from FEMA (2009) are then used to estimate the PGA at the location of each component.

\subsubsection{Hurricane Hazard Analysis}

The probabilistic and scenario hurricane hazard analysis discussed in Chapter 4 is used here. Figure 7.5 shows the steps for the probabilistic approach. The hazard curves for Charleston, New York, and Seattle are shown in Figure 7.6. Hurricane Hugo, the strongest hurricane on record to strike South Carolina, has been selected for the scenario-based hurricane hazard analysis. Hurricane track data and recorded wind speeds are obtained from the National Oceanic and Atmospheric Administration (NOAA, 2015b).

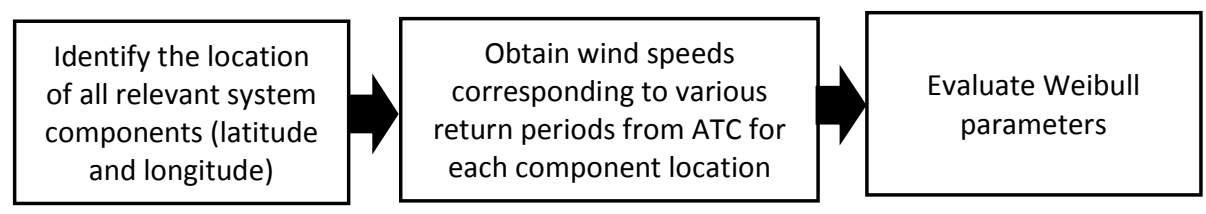

Figure 7.5 Probabilistic hurricane analysis 


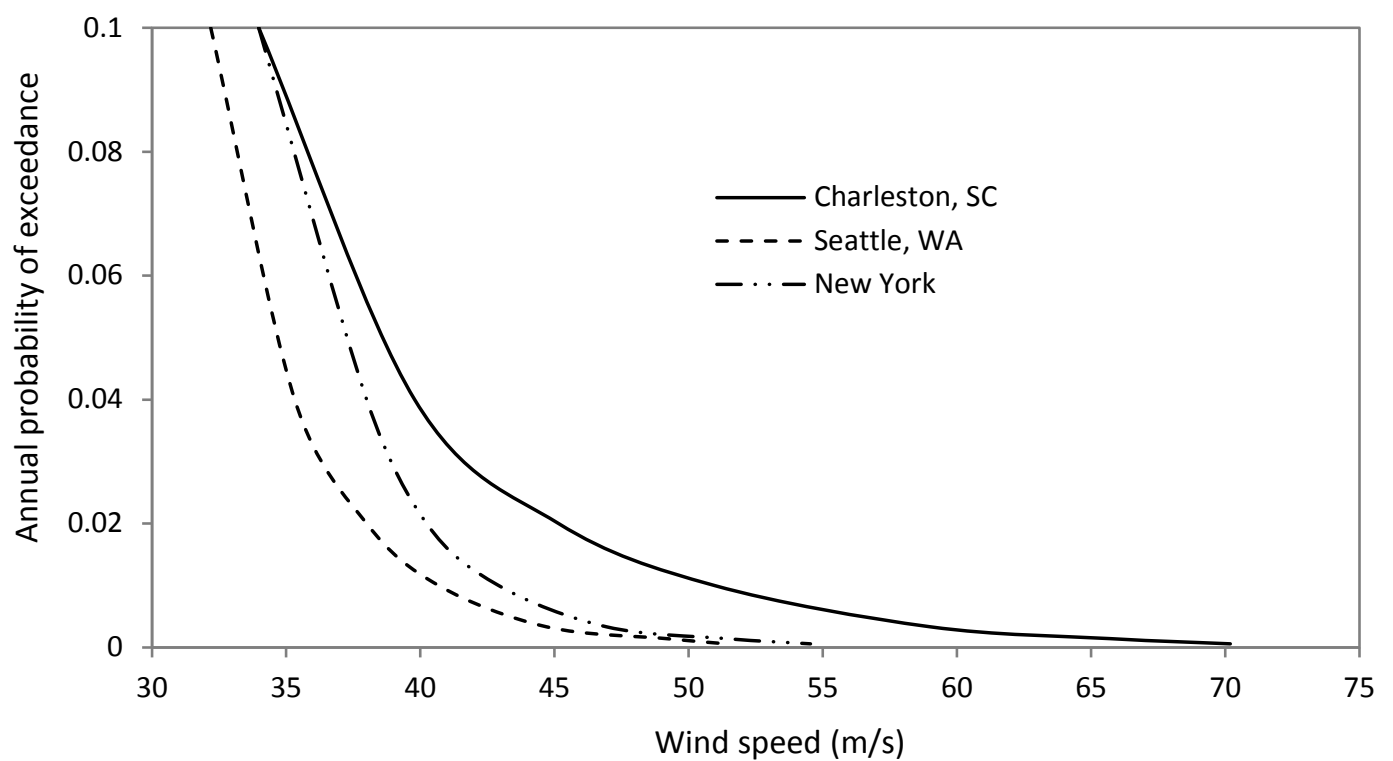

Figure 7.6 Hurricane hazard curves

Charleston $\left(32.8^{\circ} \mathrm{N} 79.9^{\circ} \mathrm{W}\right)$, New York $\left(40.71^{\circ} \mathrm{N} 74^{\circ} \mathrm{W}\right)$, and Seattle $\left(47.6^{\circ} \mathrm{N} 122.3^{\circ} \mathrm{W}\right)$

\subsection{Component Vulnerability Analysis}

The structural components of electric power systems considered in this study are the substations and transmission structures/lines. Since the objective of this study is to model multi-hazard risk assessment, the fragilities of the system components are taken from existing literature.

\subsubsection{Substation Fragility}

Due to the nature and weight of substation components, substations are rarely damaged by hurricane winds. Rather, flooding resulting from storm surge is of greater concern for substations (Brown, 2009). Since only hurricane winds are considered in this research, it 
is assumed that substations are not affected by hurricanes. Substations are however vulnerable to earthquakes due the presence of brittle components that have considerable mass (Vanzi, 1996; Eidinger \& Kempner, 2012). FEMA (2010) modeled the seismic fragility of substations subjected to seismic hazard using lognormal distribution and provided the fragility parameters. The seismic fragility parameters provided are for high voltage, medium voltage, and low voltage substations. The substations are also classified based on whether the subcomponents are anchored to resist seismic loads or not.

FEMA (2010) considered five damage states for the seismic fragility of substations. These are none, slight/minor, moderate, extensive, and complete. These damage states are defined based on the percentage of subcomponents being damaged rather than power flow within the substation. In this study, it is assumed that substations in extensive or complete damage states will lose their functionality and are considered failed (Dueñas-Osorio et al., 2007). Since exceeding extensive damage state is a prelude to exceeding complete damage state, the substations are hence considered failed in extensive damage state. The fragility parameters for extensive damage of the three classes of substations are shown in Table 7.2 (FEMA, 2010). In Chapter 8, all the damage states will be considered for evaluating the cost-effectiveness of mitigation strategies.

Table 7.2 Lognormal parameters for seismic fragility of substations with anchored components

(Damage state: extensive)

\begin{tabular}{lll}
\hline \hline Substation & Median PGA $(\mathrm{g})$ & Dispersion \\
Classification & & \\
\hline Low voltage & 0.45 & 0.45 \\
Medium voltage & 0.35 & 0.40 \\
High voltage & 0.20 & 0.35 \\
\hline
\end{tabular}

Figure 7.7 shows the seismic fragilities of the substations for the damage state of extensive damage based on the lognormal parameters in Table 7.2. The seismic fragilities of the 
substations are calculated with respect to peak ground acceleration (PGA) because facilities such as substations and distribution circuits are mostly vulnerable to PGA unless they are located in liquefiable or landslide zones in which case they will sometimes be vulnerable to peak ground displacement (PGD) (FEMA, 2010).

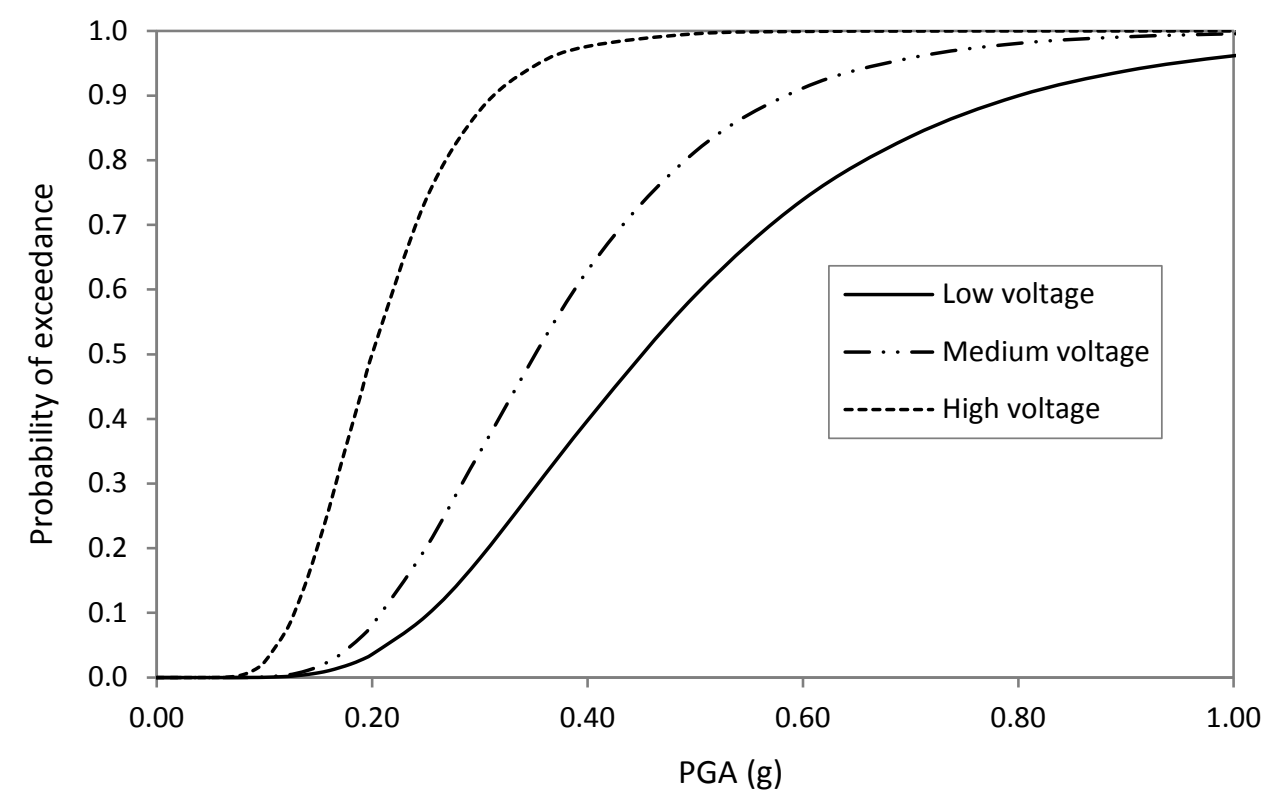

Figure 7.7 Seismic fragility curves of substations for extensive damage state

\subsubsection{Transmission Line Fragility}

Transmission towers are rarely damaged by the actual shaking of the ground during earthquakes as they are designed for severe loads such as combined wind and ice, extra loads due to the collapse of adjacent towers and so on. Instead, the damage is mostly due to foundation failures caused by landslides, ground fracture, and liquefaction. However, there is a lack of data to include such failures in analytical studies (Shinozuka et al., 2005). Therefore, it is assumed in this research that the transmission structures are not affected by earthquakes. 
The fragility of transmission line support structures subjected to wind load depends on several factors such as variability in tower types, load direction, and the potential for cascading failure. These factors make analytical fragility analysis of transmission lines quite complex. Another viable approach is through the use of empirical failure data to plot the fragility curve of transmission structures. One advantage of transmission structure fragility developed from empirical data over fragility curves developed using analytical methods is that in analytical method, it is difficult to combine several failure mechanisms such as flexural and foundation failure together. In most cases, only one failure mechanism is considered at a time in analytical fragility analysis. Fragility curves developed based on empirical data remedy this shortcoming to a greater extent.

Brown (2009) developed a fragility curve for transmission support structures subjected to hurricane winds based on 10-year storm-related damage data provided by four coastal utility companies. According to the data, a total of 1,947 transmission structures were damaged or replaced in the 10-year period. The exponential model fitted to the damage data is given by Equation (7.5). The fragility curve is shown in Figure 7.8. It should be noted that the fragility of transmission structures depends on the type of structure as well as the conductor span. The fragility function given by Equation (7.5) however did not take these factors into account. Rather, it based on general damage data collected by the utility companies. Therefore, the adoption of Equation (7.5) here is for the purpose of demonstrating the proposed framework. Accurate fragility data can be developed by individual utility companies to guide decision making.

$$
P(C<D)=\min \left\{\left[\left(2 \cdot 10^{-7}\right) e^{0.0834 \cdot v}\right], 1\right\}
$$

where $C$ is capacity; $D$ is demand; and $P(C<D)$ is the probability of failure at a given wind speed, $v$. For hurricane hazard analysis, all the structures in a single line are assumed to be subjected to the same wind speed. 


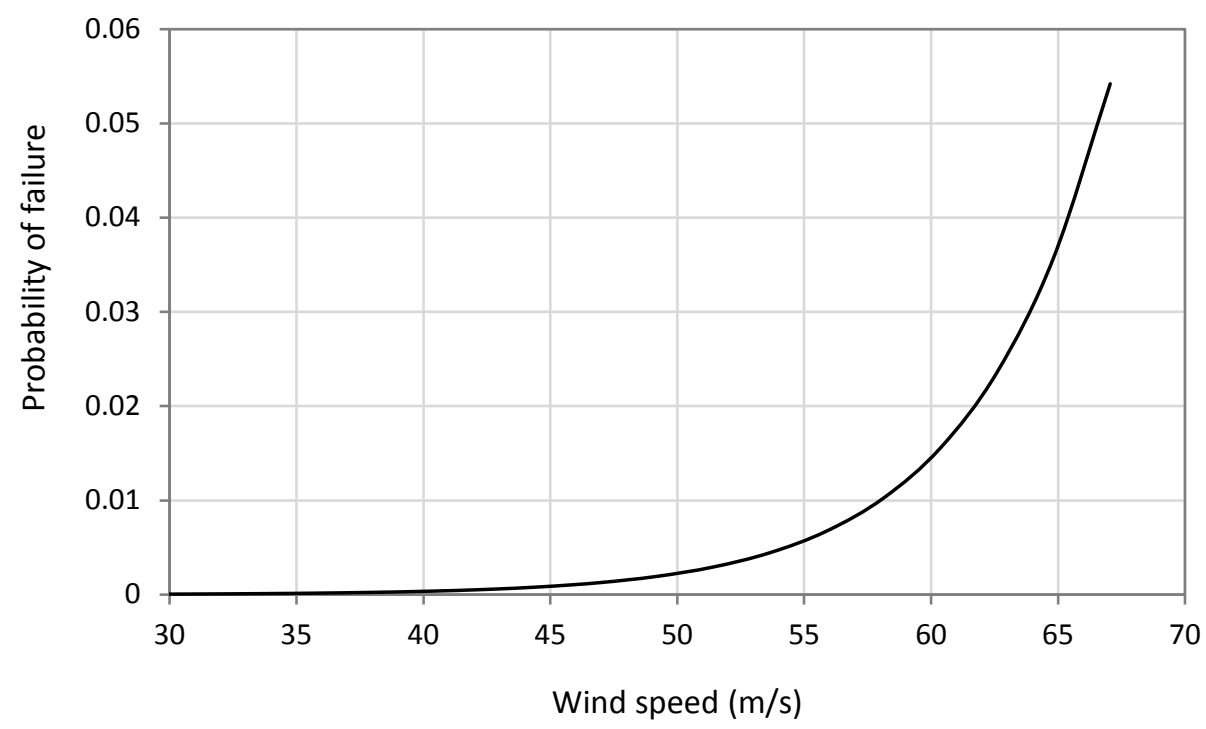

Figure 7.8 Fragility curve of transmission support structures

\subsection{Component Risk Assessment}

The seismic risk to infrastructure components using the probabilistic seismic hazard analysis is performed by convolving component fragility with the seismic hazard curve. The annual probability of exceeding a certain damage state is given by Equation (7.6).

$$
P_{A}=\int_{0}^{\infty} F_{R}(I M) \cdot\left|\frac{d \hat{v}}{d(I M)}\right| d(I M)
$$

where $P_{A}$ is the annual probability of exceeding a specified damage state; $F_{R}(I M)$ is the fragility function given a certain level of intensity measure, $I M$, which is modeled with a lognormal function for substations in this case; and $\hat{v}$ is the seismic hazard function given by Equation (7.2). 
For scenario-based seismic hazard analysis, the risk to components of an infrastructure system is defined as the probability of failure given a specific level of seismic intensity. The risk is evaluated directly from the fragility curves of the components.

Using the probabilistic hurricane analysis, the risk to infrastructure components is quantified using the annual probability of failure which is estimated by convolving the structural fragility with a hurricane wind speed model as:

$$
P_{A}=\int_{0}^{\infty} F_{R}(v) f_{v}(v) d v
$$

where $F_{R}(v)$ is the cumulative distribution function (CDF) of the structural fragility given a wind speed $v$; and $f_{v}(v)$ is the probability density function (PDF) of the hurricane wind speed.

For the scenario-based hurricane analysis, the risk to components is defined as the probability of failure given a specific level of wind speed. The risk is evaluated directly from the fragility curves of the components.

The results of the components risk analysis are shown in Figure 7.9 for Charleston. It can be seen from Figure 7.9(a) that the seismic risk to substations increases with increasing voltage rating. The high voltage gate stations $(\mathrm{G} 1-\mathrm{G} 8)$ are more vulnerable while the low voltage substations are generally the least vulnerable. This can also be inferred from the fragility curves of the substations in Figure 7.7. For the seismic risk result shown in Figure 7.9(b) based on the scenario earthquake, the seismic risk depends on both the substation voltage rating as well as the location of each substation relative to the epicenter of the earthquake. The component fragility results for the transmission lines subjected to hurricane winds are given in Figure 7.9(c) \& Figure 7.9(d). The fragilities of the lines depend both on location and length of lines. 


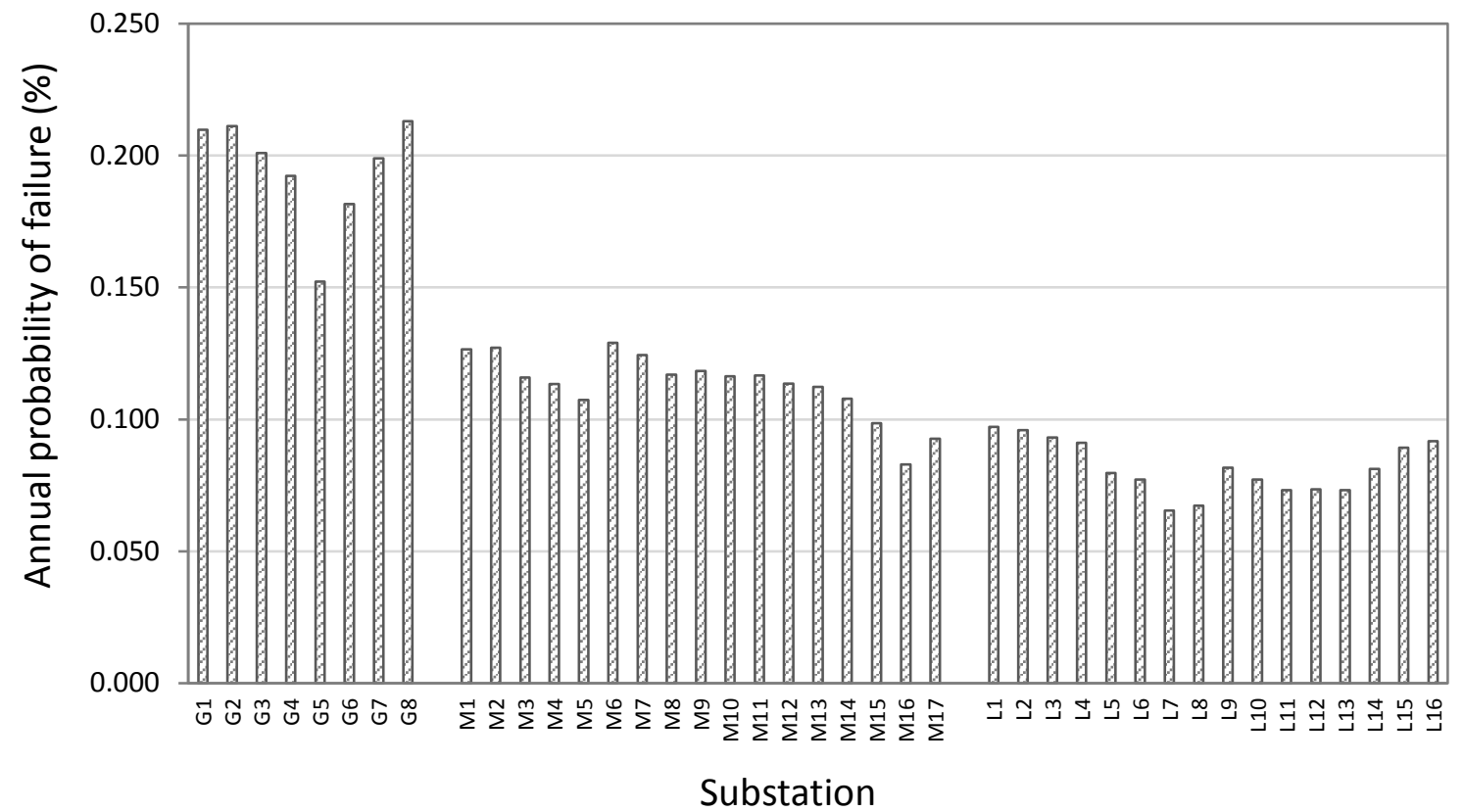

(a)

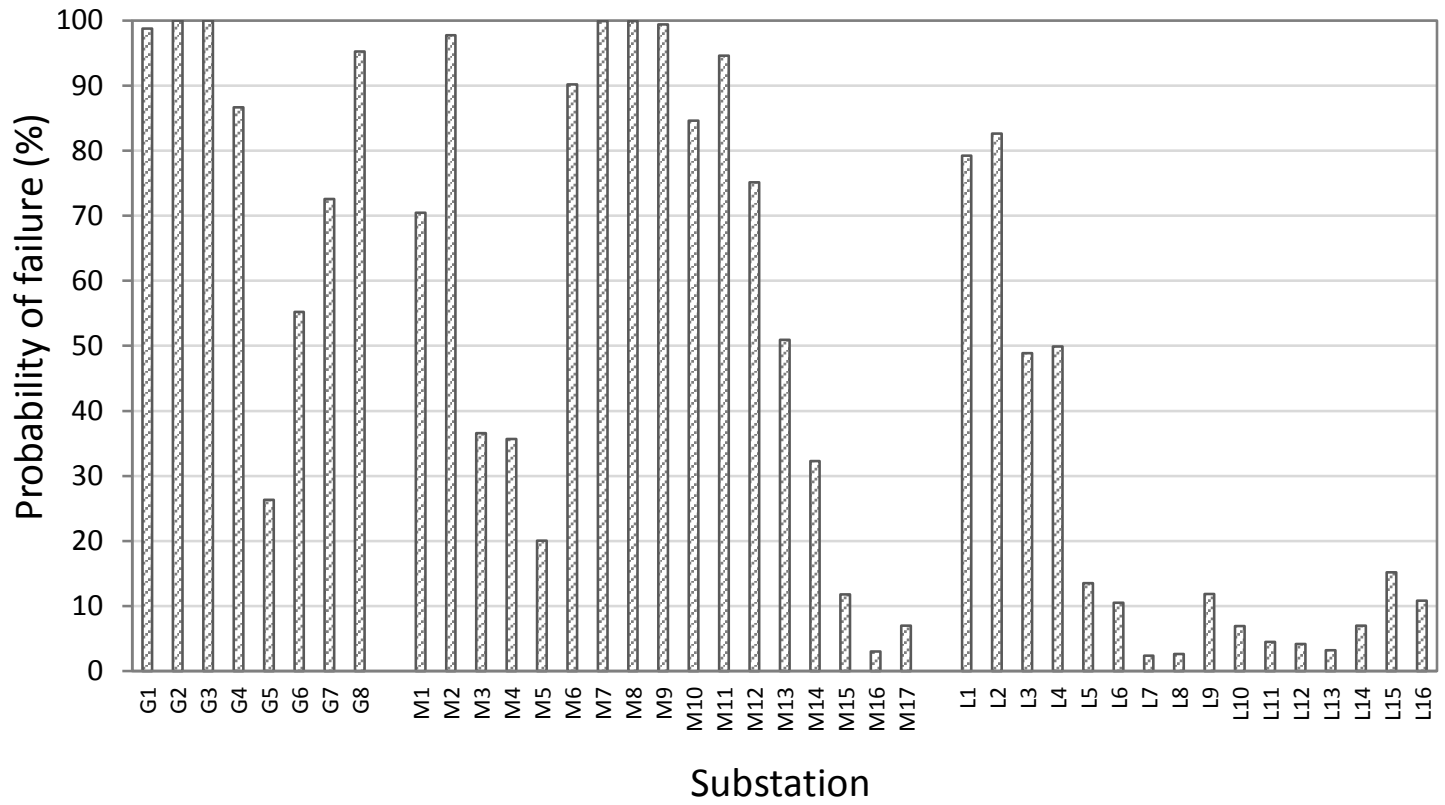

(b) 


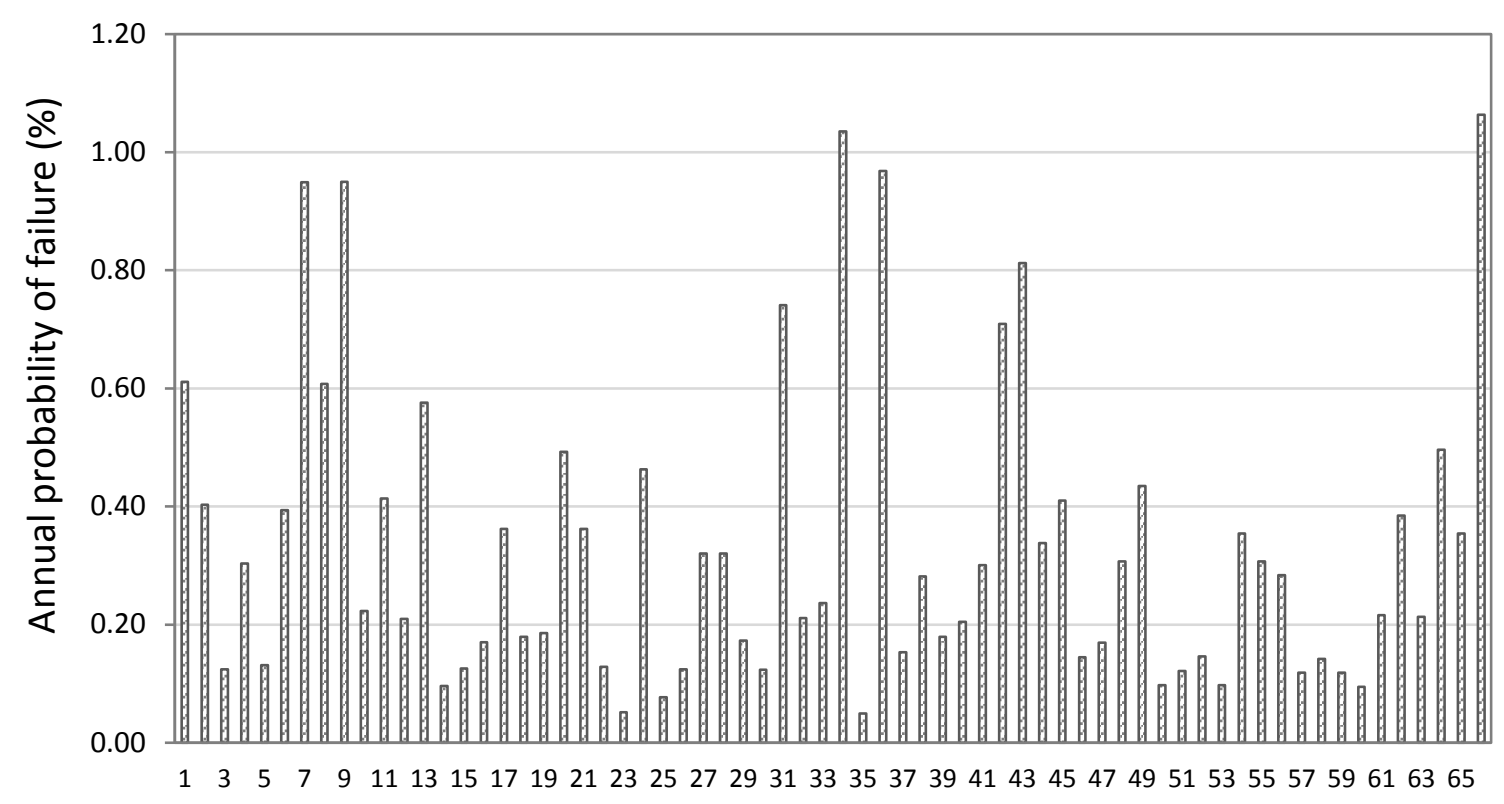

Transmission line

(c)

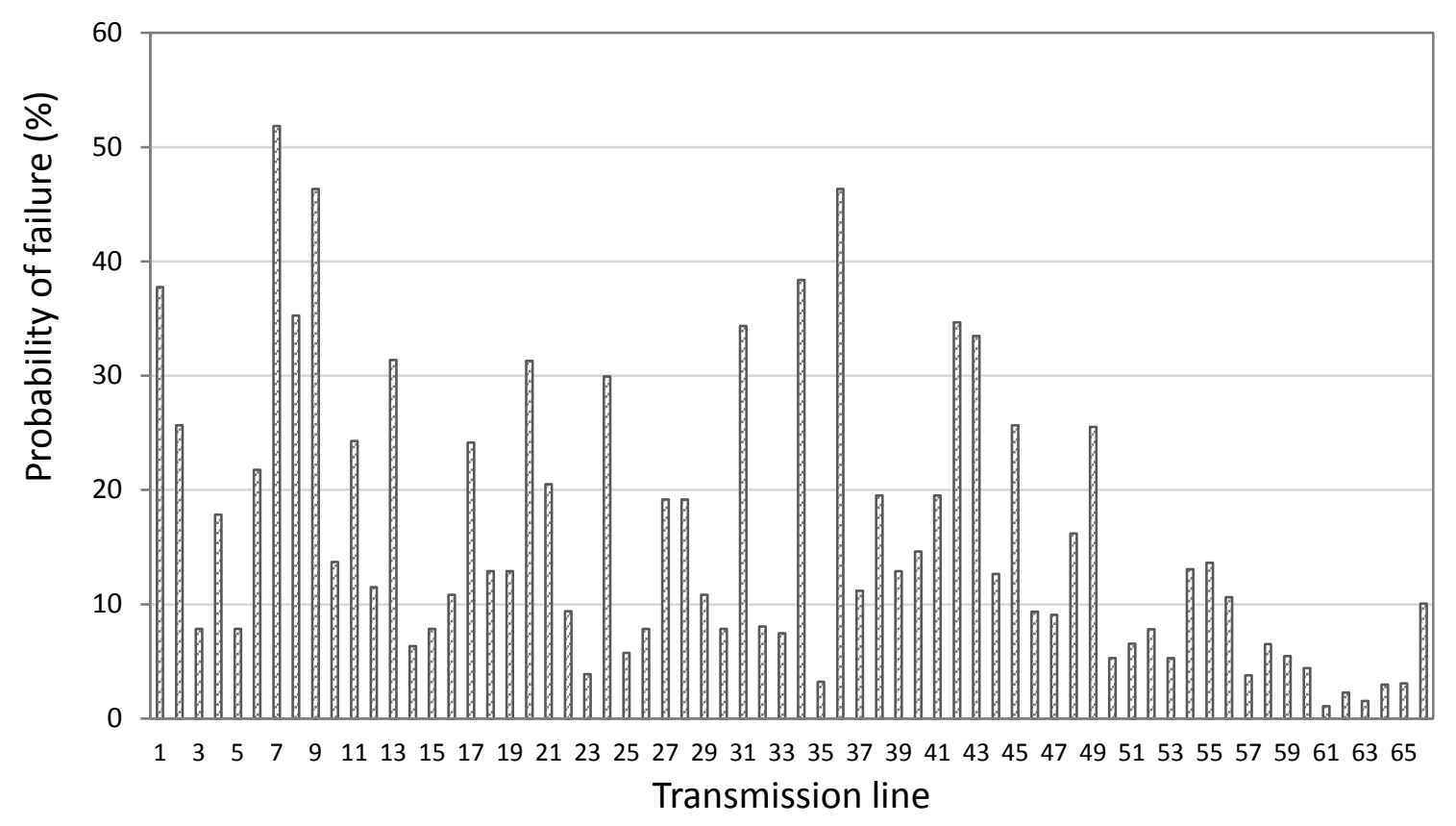

(d)

Figure 7.9 Component risk results for Charleston

(a) PSHA, (b) scenario earthquake, (c) probabilistic hurricane analysis, and (d) scenario hurricane 


\subsection{System Reliability Results}

System reliability depends on the accessibility of each substation from supply gate stations. Accessibility here is modeled as the probability of power not being delivered to a substation which is discussed in Chapter 3. The results of the accessibility of the demand substations are given in Figure 7.10 for Charleston. It can be seen from Figure 7.10(b) that a lot of the substations will not be able to receive power following the scenario earthquake as most of the supply stations (G1 to G8) will likely fail following the earthquake as can be seen from their fragilities in Figure 7.9(b).

From Figure 7.10(c) \& Figure 7.10(d), it can be seen that accessibility of the substations under hurricane hazard depends largely on the topology of the transmission lines supplying power to each substation. For instance, M5 has one of the highest probability of power not delivered in Figure 7.10(c) because, despite the fact that it can be supplied by 5 gate stations through several routes, its accessibility relies largely on the reliability of line 16. 


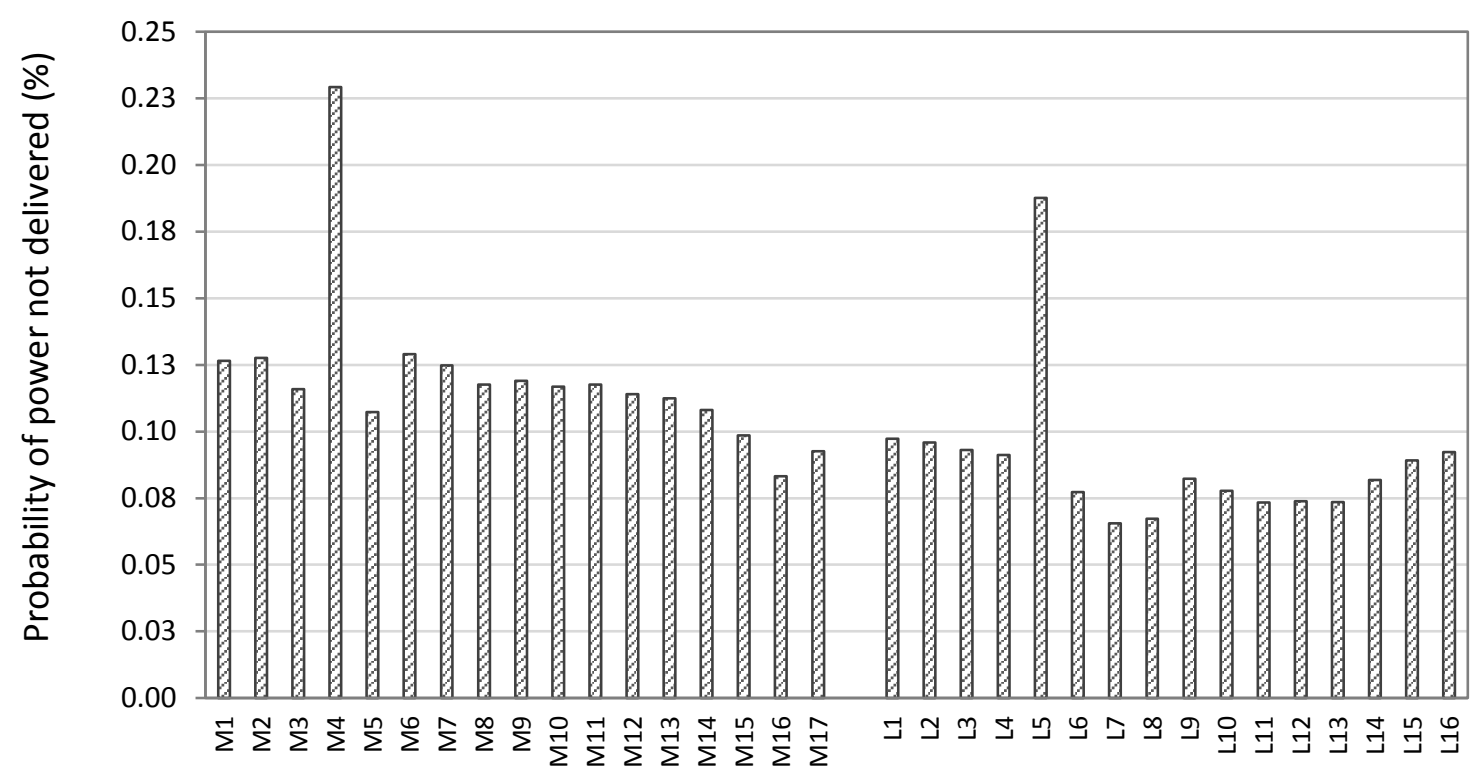

Substation

(a)

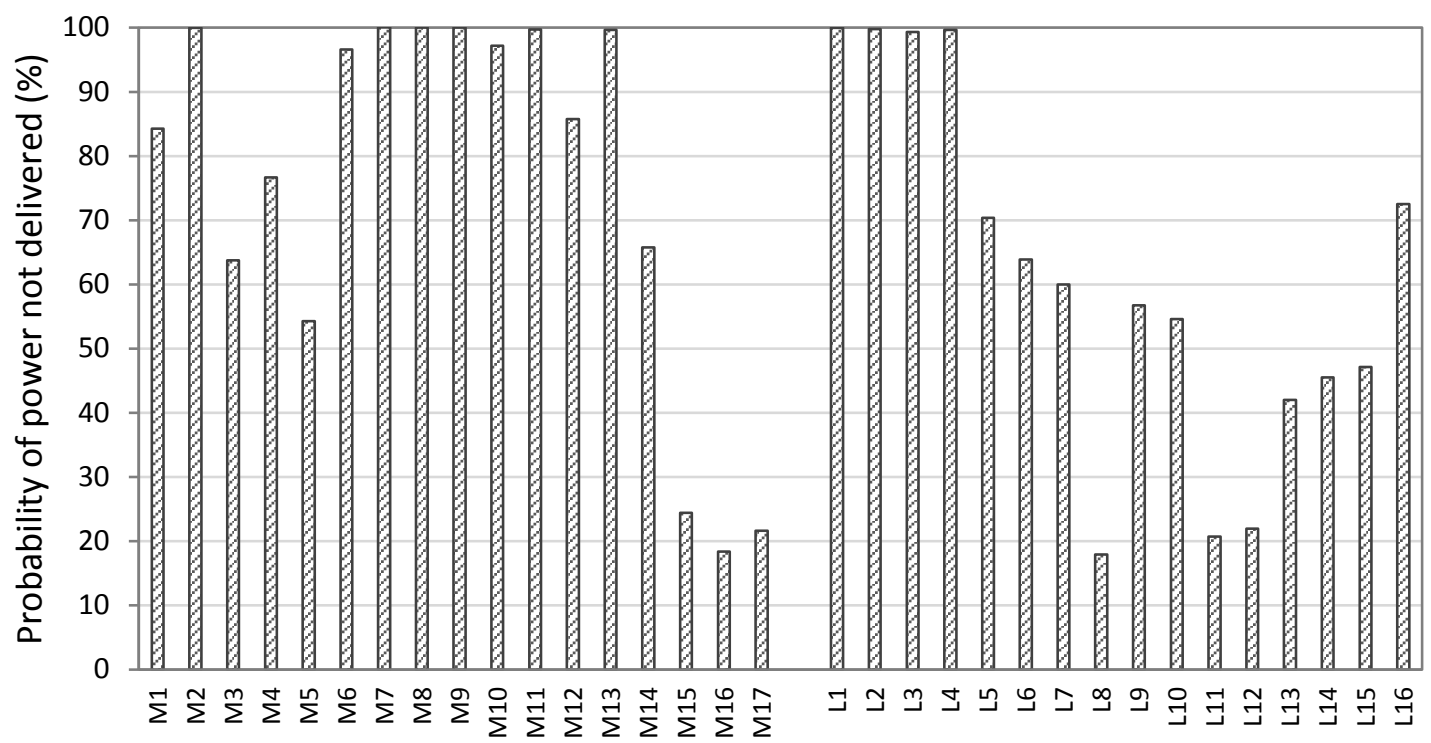

Substation

(b) 


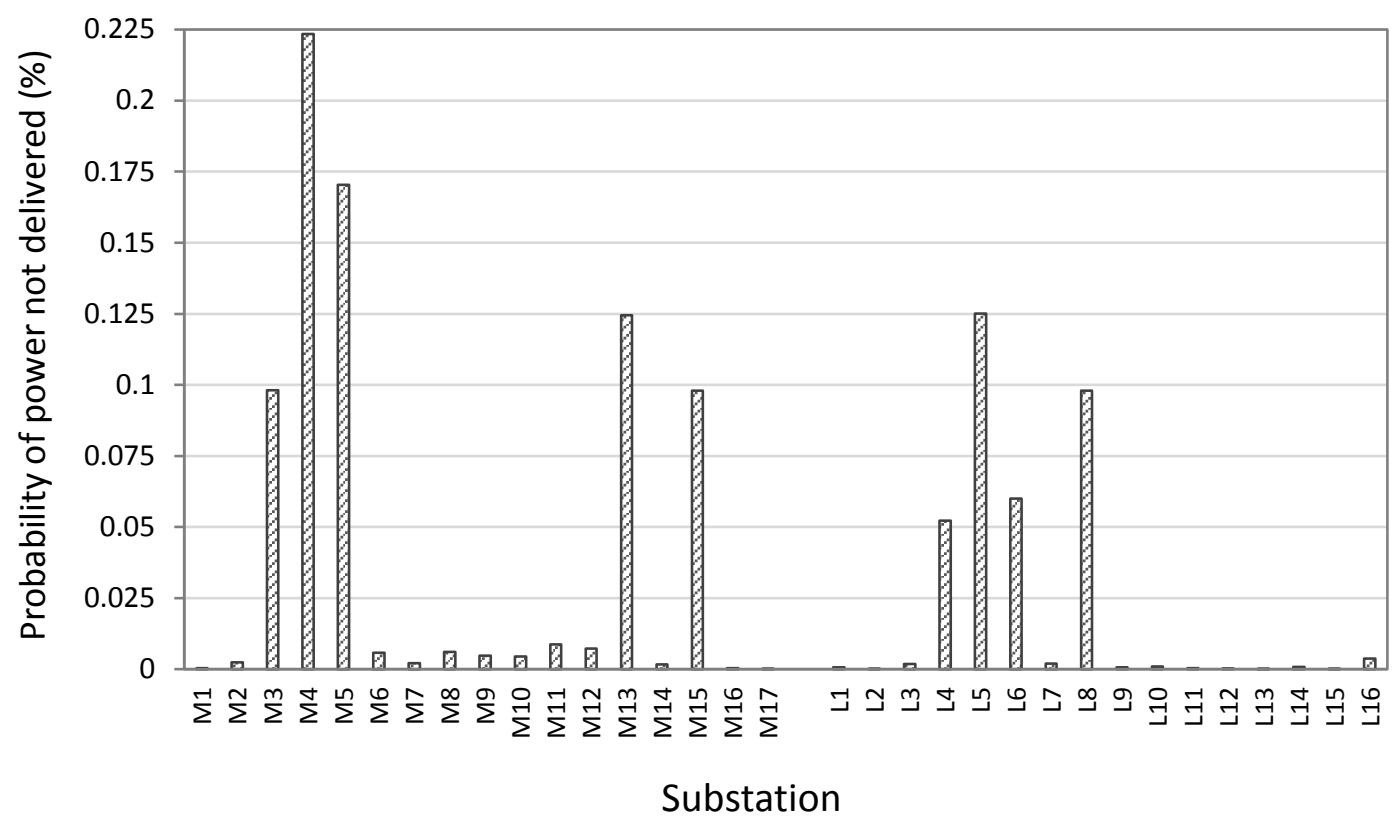

(c)

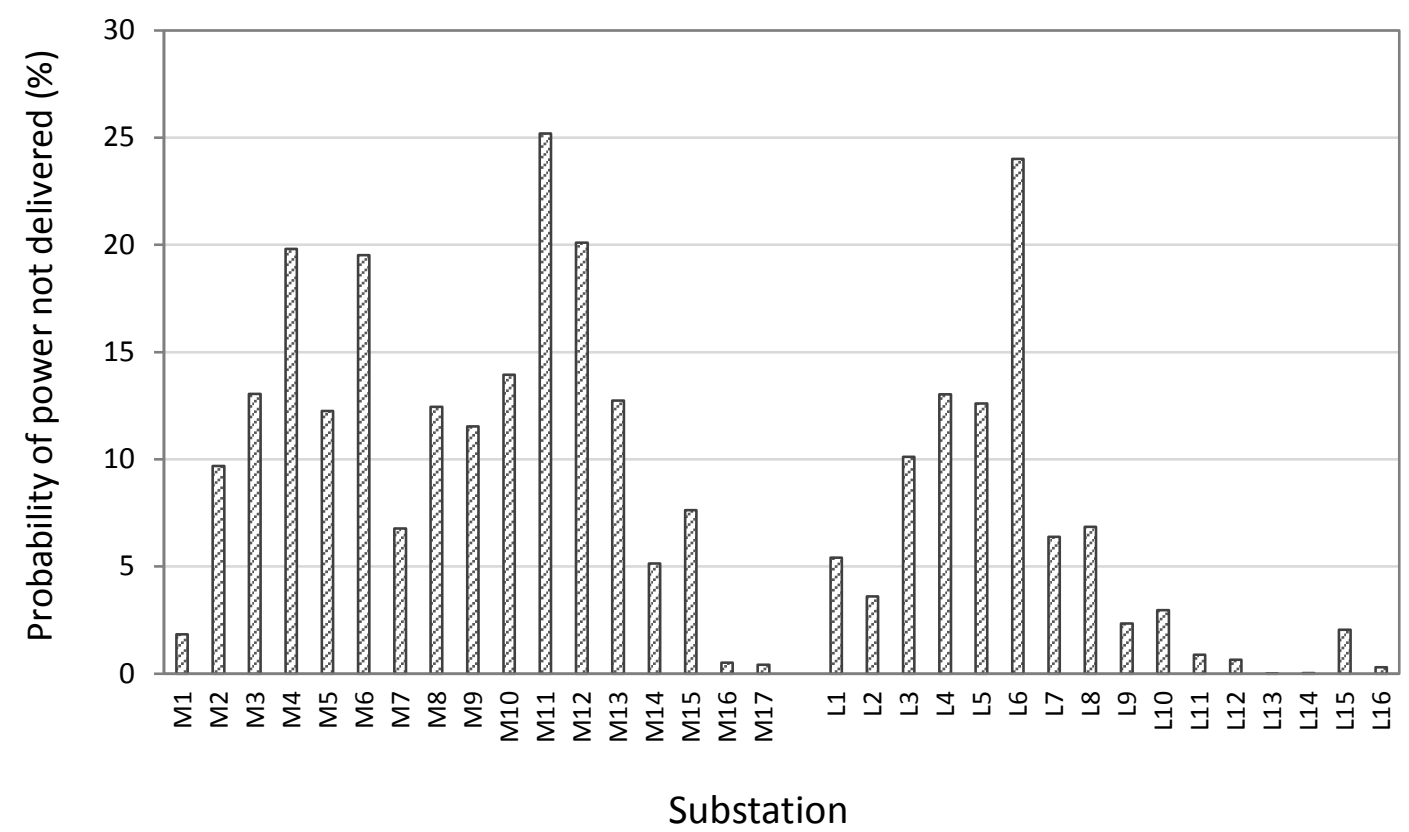

(d)

Figure 7.10 Accessibility of demand substations in Charleston

(a) PSHA, (b) scenario earthquake, (c) probabilistic hurricane analysis, and (d) scenario hurricane 
The result of the system reliability analysis is summarized in Table 7.3 for Charleston. It can be seen from the table that for both the probabilistic seismic and hurricane hazard analysis, the system reliability is similar to probabilistic seismic hazard analysis resulting in a slightly lower reliability. Considering the scenario-based approach, on the other hand, the scenario earthquake has a much more devastating impact on system reliability $(30.27 \%)$ than the scenario hurricane $(90.93 \%)$. This can be attributed to the fragility of system components under both hazards. The gate stations and substations have much higher probabilities of failure under the giving earthquake than the transmission lines have under the giving hurricane as seen in Figure 7.9(b) \& Figure 7.9(d). Another reason has to do with the fact that the failure of a transmission line will have far less impact on system reliability than the failure of a substation or most especially supply gate stations. Further discussion on comparison of the two hazards will follow in the next section.

Table 7.3 Electric power system reliability results for Charleston

\begin{tabular}{lll}
\hline \hline Hazard Analysis Type & Hazard Description & $\begin{array}{l}\text { System } \\
\text { Reliability (\%) }\end{array}$ \\
\hline $\begin{array}{l}\text { Probabilistic seismic hazard } \\
\text { analysis (annual risk) }\end{array}$ & $\begin{array}{l}\text { Aggregated effect of all possible } \\
\text { seismic hazard levels }\end{array}$ & 99.89 \\
$\begin{array}{l}\text { Scenario-based seismic hazard } \\
\text { analysis (risk due to one event) }\end{array}$ & $\begin{array}{l}\text { (.3 magnitude earthquake (strongest } \\
\text { earthquake on record to hit SC) }\end{array}$ & 30.27 \\
$\begin{array}{l}\text { Probabilistic hurricane hazard } \\
\text { analysis (annual risk) }\end{array}$ & $\begin{array}{l}\text { Aggregated effect of all possible } \\
\text { hurricane hazard levels }\end{array}$ & 99.96 \\
$\begin{array}{l}\text { Scenario-based hurricane hazard } \\
\text { analysis (risk due to one event) }\end{array}$ & $\begin{array}{l}\text { Category 4 hurricane Hugo (strongest } \\
\text { hurricane on record to hit SC) }\end{array}$ & 90.93 \\
\hline
\end{tabular}




\subsection{Multi-Hazard Risk Assessment}

\subsubsection{Risk Comparison Based on Risk Curves}

For comparison of seismic and hurricane risks, it is imperative to use some kind of a common risk indicator. In this research, system reliability is used. Here, the concept of a multi-hazard risk curve is introduced. Such a risk curve shows a plot of system reliabilities against corresponding return periods (or exceedance probabilities). This allows direct quantitative comparison of the risks for the range of return periods covered by both hazards. To construct the multi-hazard risk curves, several return periods (or annual probabilities of exceeding various hazard levels) are selected and their corresponding PGA and wind speed at locations of all substations and lines calculated. System reliability corresponding to each hazard level (PGA and wind speed) is then evaluated. The multi-hazard risk curves of the power system in Charleston, New York, and Seattle are shown in Figure 7.11. 


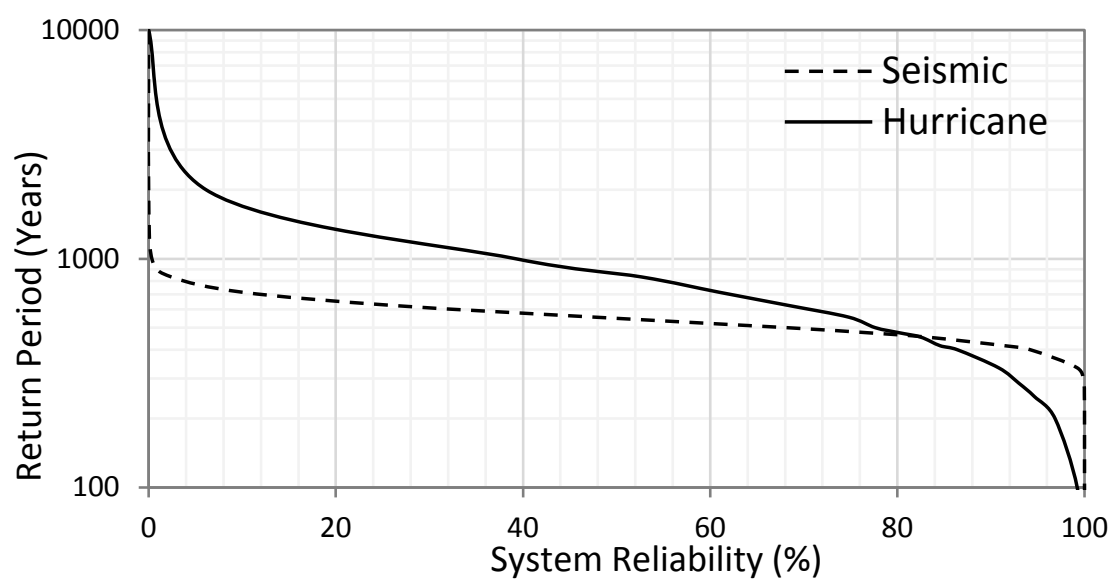

(a)

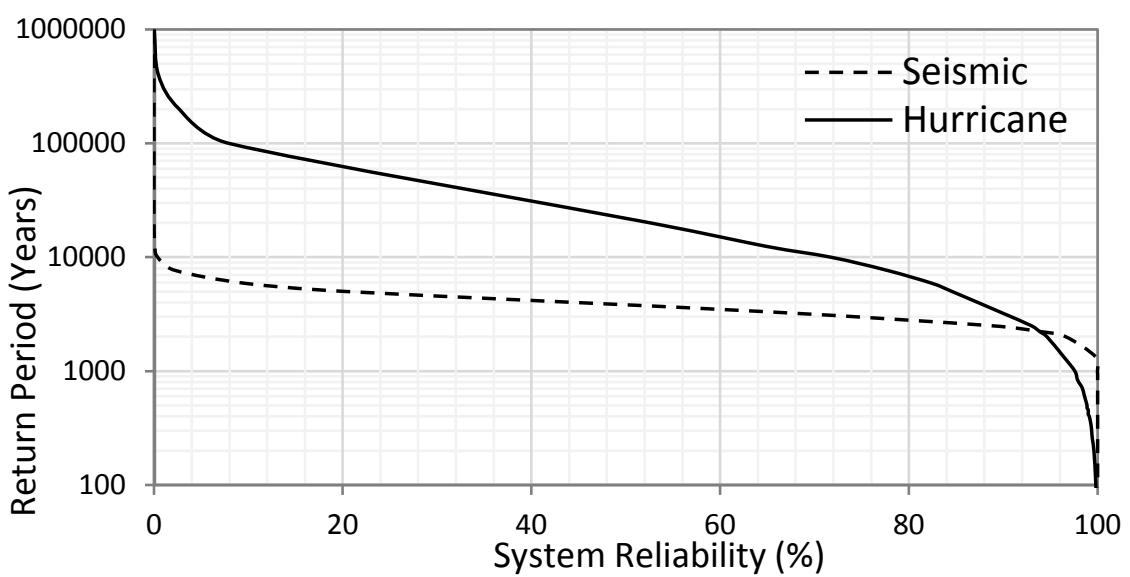

(b)

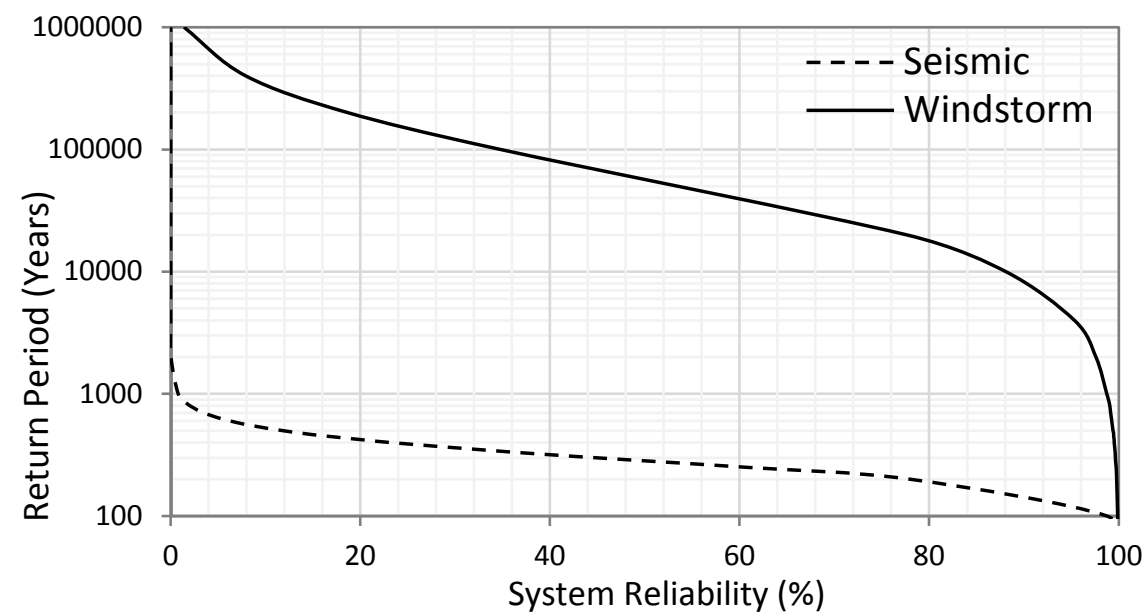

(c)

Figure 7.11 Multi-hazard risk curves

(a) Charleston, (b) New York, (c) Seattle 
From Figure 7.11(a), it can be seen that at higher return periods (lower exceedance probabilities) greater than about 460 years, the risk is clearly dominated by seismic hazard in Charleston. For instance, at a return period of 700 years, the system reliability due to seismic hazard is $12 \%$ compared to $62 \%$ due to hurricane hazard. It can also be seen that a 2000-year return period earthquake will cause a complete shutdown of the system $(0 \%$ reliability) as compared to a 10,000-year return period hurricane that will cause the same impact.

For more frequent events with return periods less than 460 years, it can be seen from Figure 7.11(a) that hurricane hazard has more impact on the system than the seismic hazard. Both hazards have the same effect on system reliability at a return period of about 460 years. From Figure 7.11(b), it can be seen that the pattern in New York is similar to that in Charleston with seismic hazard dominating the risk at return periods higher than 2,200 years. Compared to Charleston, however, it can be seen that both the seismic and hurricane risks are lower in New York.

Looking at the multi-hazard risk curve for Seattle in Figure 7.11(c), it can be seen that earthquake is clearly the dominant hazard at all return periods. Windstorms seem to pose very little risk to the system in this location. For instance, at a return period of 1000 years, the system reliability is about $1 \%$ for the corresponding seismic hazard level while it is $98 \%$ for windstorms. At lower return periods, 200 years, for example, the system reliability is $78 \%$ and $99 \%$ for seismic and storm hazards, respectively.

The information gathered from the multi-hazard risk curves is valuable in decision making regarding risk mitigation investment as it gives information on the impact of both lowprobability high-consequence events as well as frequent events on system reliability (Ellingwood \& Wen, 2005).

The wind speeds used to develop the hurricane curves in Figure 7.11 were obtained from ATC (2015) as mentioned earlier, which were developed based on hurricane simulations 
by Vickery et al. (2000b) in which 20,000 hurricane years were simulated. Therefore, it should be noted that the maximum simulated year of 1,000,000 years shown in Figure 7.11 is for demonstrating the change of system reliability vs return period of seismic and hurricane hazards.

Risk comparison can also be made based on stipulated design hazard level. The design wind speed is the wind speed with a return period of 50 years ( $2 \%$ exceedance annually) as suggested by ASCE-74 (2009). The design PGA is the PGA with traditionally a return period of 475 years (ASCE, 2005; Li \& Ellingwood, 2009). The design hazard levels and the corresponding system reliabilities are shown in Table 7.4. Note that the PGA and wind speeds in Table 4 are those for the locations of G1 and line 13, respectively, and are shown to give an indication of the general variation of the hazards in the three locations.

It can be seen from Table 7.4 that for Charleston, the design seismic hazard level has a higher impact than hurricane hazard with corresponding system reliabilities of $76.8 \%$ and $99.8 \%$, respectively. Both design hazard levels have a similar impact on the system in New York while, in Seattle, design seismic hazard level has far greater impact than hurricane hazard with corresponding system reliabilities of $13.9 \%$ and $99.9 \%$, respectively.

Note that the variation of system reliability between the three locations especially when considering seismic hazard is the fact that while design hazard levels vary (for example, design PGA is $0.303 \mathrm{~g}$ in Seattle as compared to $0.042 \mathrm{~g}$ in New York), the power system, in this case, is exactly the same in all three locations. In practice, structural components of the system will be designed for the appropriate hazard level in each location. 
Table 7.4 Multi-hazard risk comparison based on stipulated design hazard level (PGA and wind speed are for location of G1 and line 13, respectively)

\begin{tabular}{lllll}
\hline \hline \multirow{2}{*}{ Location } & Seismic Hazard & \multicolumn{3}{l}{ Hurricane Hazard } \\
\cline { 2 - 5 } & $\begin{array}{l}\text { PGA }(\mathrm{g})(475-\mathrm{yr} \\
\text { return period) }\end{array}$ & $\begin{array}{l}\text { System } \\
\text { Reliability }\end{array}$ & $\begin{array}{l}\text { Wind }(\mathrm{m} / \mathrm{s})(50-\mathrm{yr} \\
\text { return period) }\end{array}$ & $\begin{array}{l}\text { System } \\
\text { Reliability }\end{array}$ \\
\hline Charleston & 0.199 & 76.8 & 43 & 99.8 \\
New York & 0.042 & 100 & 39 & 99.9 \\
Seattle & 0.303 & 13.9 & 37 & 99.9 \\
\hline
\end{tabular}

\subsubsection{Multi-Hazard Risk Based on Annual Probability of System Failure}

The comparisons based on multi-hazard risk curves in the previous section is conditional, as the system reliability evaluations are based on scenario-based hazard analysis. Here, the comparison is made based on the aggregated effect of all possible hazard levels which is the basis for probabilistic hazard analysis. The system reliability results calculated based on the annual probability of damage to system components in the three locations are given in Table 7.5. The annual probability of system failure, defined as the complement of system reliability is plotted in Figure 7.12. The results show that in Charleston and Seattle, seismic hazard has a greater effect on the annual measure of system reliability, unlike in New York where hurricane hazard has a slightly higher effect. Considering the combined effect of both hazards, the system has a higher annual probability of failure in Seattle followed by Charleston and New York.

Even though hurricanes and earthquakes are independent non-concurrent hazards, annualizing the risk (measured as system unreliability in this case) allows for the summation of the risks due to both hazards. For example, in the case of Charleston, there is a 0.11 annual probability of system failure due to earthquakes and 0.04 annual probability of system failure due to hurricanes. The total annual probability of system 
failure due to both hazards is thus 0.15 . It should be noted that the risks due to both hazards cannot be summed in the case of comparison based on scenario hazard.

Table 7.5 Multi-hazard risk comparison based on annual probability of damage of components

\begin{tabular}{lllllll}
\hline \hline \multirow{2}{*}{ Risk Indicator } & \multicolumn{2}{l}{ Charleston } & New York & \multicolumn{2}{l}{ Seattle } & \\
\cline { 2 - 6 } & Seismic & Hurricane & Seismic & Hurricane & Seismic & Hurricane \\
& & & & & & \\
\hline System reliability & 99.89 & 99.96 & 99.98 & 99.97 & 99.80 & 99.97 \\
$(\%)$ & & & & & & \\
\hline
\end{tabular}

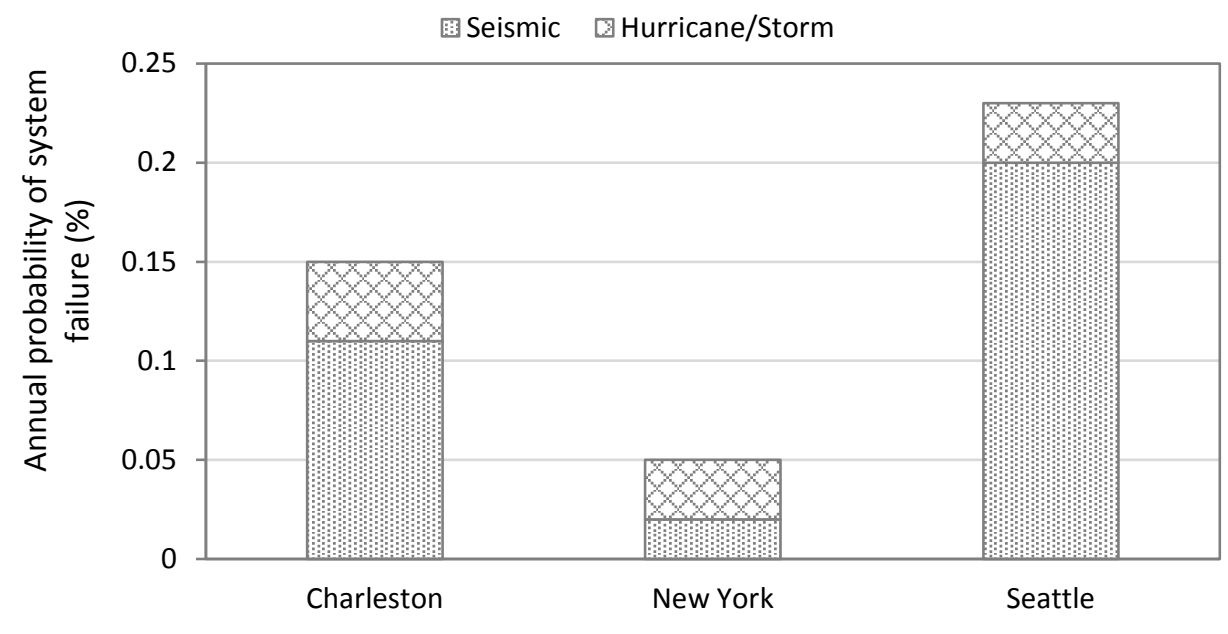

Figure 7.12 Annual probability of system failure

The similarity in the impact of the two hazards on system reliability in Table 7.5 is because all hazard levels are weighted by the probabilities of their occurrence. Consequently, even though a high magnitude earthquake can cause severe damage to the system, for example, the probability of its occurrence is low. Similarly, a low-intensity hurricane with a high probability of occurrence will cause slight damage to the system. Note that the annual 
probability of system failure is "by average", which means a hazard event could dramatically change it as seen in the scenario-based result in Table 7.3.

\subsection{Conclusions}

A multi-hazard risk assessment framework has been presented in this chapter for considering the impact of seismic and hurricane hazards on electric power systems. A more comprehensive risk assessment that takes into account the potential impact of all possible natural hazards on power systems will help to guide pre-disaster preparation as well as decision making regarding cost-effective mitigation strategies. A notional electric power system assumed to be located in Charleston, SC, New York, NY, and Seattle, WA was used to demonstrate the proposed framework. Multi-hazard risk curves developed using a topological-based system reliability indicator were used to compare the two hazards. Furthermore, system reliability evaluated based on stipulated design hazard levels as well as the annual probability of damage to structural components of the system were also used for risk comparison.

The case study considered shows that multi-hazard risk assessment enables the comparison and/or aggregation of different risks to electric power systems and can reveal the contribution of each hazard to the overall risk to the system. The case study also illustrates the importance of considering low-probability high-consequence events in disaster mitigation decisions. Based on the results obtained, mitigation efforts should be considered to reduce the potential impact of such events. However, the cost-effectiveness of deploying such mitigation measures should be evaluated and is the topic for the next chapter. 


\section{Multi-Hazard Risk Mitigation Framework ${ }^{5}$}

\subsection{Introduction}

Decision making regarding mitigation of multiple hazards differs from that of single hazard mitigation in the sense that before the level or type of mitigation strategy is selected, a decision needs to be made on which of the various competing hazards deserves greater attention. This is compounded by the fact that there are limited resources available for mitigation of risks from competing hazards. For example, by 2020, the investment gaps for distribution and transmission infrastructure are estimated to be $\$ 57$ billion and $\$ 37$ billion, respectively (ASCE, 2013). Therefore, both identification and prioritization of risks are essential for decision making regarding investment in mitigation strategies.

Multi-hazard assessment and mitigation were investigated for residential construction (Li \& Ellingwood, 2009), bridges (Kameshwar \& Padgett, 2014), and commercial buildings (Wen \& Kang, 2001). However, there have not been studies on mitigation strategies for electric power systems. Existing studies on mitigation strategies for electric power systems focus on single hazards (e.g. Romero et al. (2015), Chang (2003), Shinozuka et al. (2005), Salman et al. (2015)). However, investment in long-term mitigation of risks needs to take into account all possible hazards that can affect the system over its entire lifespan. Therefore, there is a need to investigate the cost-effectiveness of mitigation strategies in reducing the overall risks to infrastructure that are vulnerable to multiple hazards. This requires a comprehensive multi-hazard risk-based assessment.

As resources for risk mitigation strategies are limited, any framework for multi-hazard risk mitigation should attempt to identify risk-critical parts of a system that when strengthened, will have a greater impact on overall system performance. To determine critical parts of a

\footnotetext{
${ }^{5}$ A version of this chapter has been submitted to Reliability Engineering \& System Safety.
} 
system for resource allocation, some form of component importance measure is required. This, in turn, requires evaluating a measure of system reliability.

This chapter presents the second part of the framework in Chapter 7 to study the effectiveness of multi-hazard risk mitigation strategies for electric power systems subjected to hurricanes and earthquakes. Unlike in Chapter 7, probabilistic and scenario-based hazard analysis are combined into a probabilistically weighted deterministic hazard scenarios model that consider both spatial variation in hazard intensity as well as probabilistic nature of hazard occurrence. A new component importance measure that considers multi-element failure in a networked system is also proposed. Cost-effectiveness of various mitigation strategies is investigated through life cycle cost analysis. The framework is demonstrated using the same notional electric power network in Chapter 7. However, in this case, two locations: Charleston, SC, and New York, NY are used. Seattle is not considered here due to lack of data to perform hurricane simulation which is necessary for the probabilistically weighted deterministic scenarios approach.

\subsection{Hazard Analysis}

As discussed earlier, application of probabilistic hazard analysis to spatially distributed infrastructure systems has been shown to be limited (Adachi \& Ellingwood, 2010). This is because the spatial variation of intensity for a severe hazard event is lost in the aggregation process of probabilistic analysis. Furthermore, in carrying out cost-effectiveness analysis, estimation of revenue loss and direct economic losses to society due to power outage depends on power outage duration following a specific event. Scenario-based approach, on the other hand, cannot capture all possible hazard levels in an area. In such a case, a probabilistically weighted deterministic hazard scenarios approach can be employed. This entails selecting a suite of hazard events under which system performance can be measured. The risk assessment is then carried out by weighing each hazard event with its respective 
probability of occurrence. Consequently, the probabilistic nature of hazard occurrence and spatial variation of hazard intensities are reconciled.

\subsubsection{Seismic Hazard Analysis}

To model the seismic risk in the two chosen locations, a suite of earthquake scenarios with their corresponding annual probabilities of occurrence is required. The aim is to select enough earthquake scenarios to closely replicate the seismic hazard curves obtained from USGS (2015a). The scenario earthquakes, in this case, are selected from a catalog of earthquakes for Central and Eastern United States (CEUS) compiled in the Central and Eastern United States - Seismic Source Characterization (CEUS - SSC) for Nuclear Facilities report (EPRI et al., 2012). The scenarios are selected to represent all seismic source zones in the area, as well as the range of damaging earthquakes that are possible in the area as suggested by Chang et al. (2000). Nine and eight scenario earthquakes from a shortlist are selected for Charleston and New York, respectively, from the CEUS-SSC report. The selection is made so as to cover all possible hazard levels as accurate as possible and also to reduce computational effort.

The selected scenario earthquakes from CEUS-SSC are from historical records and might not represent the entire risk in a given location, i.e., earthquake events of higher magnitude than those recorded are possible. Therefore, the maximum probable earthquake (MPE) from de-aggregation analysis of earthquakes from USGS (2008) at a risk level of $2 \%$ in 50 years is also included in the list of scenario earthquakes for Charleston. The MPE corresponding to a risk level of $2 \%$ in 50 years and $1 \%$ in 200 years are selected for New York. The MPEs account for future events of higher magnitudes as MPE is defined as the largest predicted earthquake a fault is capable of generating (Robert, 2002). This makes a total of 10 scenario earthquakes for both locations as shown in Table 8.1.

For each scenario earthquake, the peak ground acceleration (PGA) at any location within the power network is evaluated using the attenuation relationship developed by Toro et al. 
(1997). An initial annual probability of exceeding the calculated PGA level is assigned so as to closely match the hazard curve from USGS (2015a) at a particular site. The annual probability of exceedance is then revised iteratively to minimize the error between the actual hazard curve from USGS (2015a) and the hazard curve based on the chosen scenario earthquakes.

The locations of the 8 gate stations (G1 - G8) are used as control points to adjust the annual probabilities of exceedance. This is because if only one location is used to assign the probabilities, the resulting scenarios and their corresponding probabilities might not accurately model the hazard curves in other locations. Using 8 control points will ensure that the resulting scenarios and their corresponding probabilities can model the hazard in the entire area covered by the electric power system. Figure 8.1 shows the hazards curves for the locations of G1, G5, and G8 for Charleston and New York. It can be seen that the hazard curves based on the selected scenario earthquakes match the actual hazard curves from USGS (2015a).

Note that the annual probability of exceedance assigned to each seismic event or PGA level is cumulative of the probabilities of occurrence of events that will produce the same level of PGA or higher. Therefore, the probability of occurrence of an event is found by subtracting the appropriate annual probabilities of exceedance as shown in Table 8.1. 
Table 8.1 Selected scenario earthquakes and their annual probabilities of occurrence

\begin{tabular}{|c|c|c|c|c|c|c|c|c|c|}
\hline \multicolumn{5}{|c|}{ Charleston } & \multicolumn{5}{|c|}{ New York } \\
\hline \multicolumn{2}{|c|}{ Epicenter } & \multirow{2}{*}{ Magnitude } & \multirow{2}{*}{$\begin{array}{l}\text { Annual prob. } \\
\text { of exceedance }\end{array}$} & \multirow{2}{*}{$\begin{array}{l}\text { Annual prob. } \\
\text { of occurrence }\end{array}$} & \multicolumn{2}{|c|}{ Epicenter } & \multirow{2}{*}{ Magnitude } & \multirow{2}{*}{$\begin{array}{l}\text { Annual prob. } \\
\text { of exceedance }\end{array}$} & \multirow{2}{*}{$\begin{array}{c}\text { Annual prob. of } \\
\text { occurrence }\end{array}$} \\
\hline Lat. & Long. & & & & Lat. & Long. & & & \\
\hline 33.75 & 81.38 & 4.5 & 0.021 & 0.006 & 40.79 & 74.25 & 3.1 & 0.0225 & 0.0085 \\
\hline 34.01 & 80.8 & 5.2 & 0.015 & 0.006 & 40.8 & 74 & 2.7 & 0.0140 & 0.0010 \\
\hline 32.9 & 80 & 4.0 & 0.009 & 0.003 & 40.1 & 74.5 & 4.4 & 0.0130 & 0.0015 \\
\hline 33.4 & 80.42 & 5.3 & 0.006 & 0.002 & 40.46 & 74.3 & 4.1 & 0.0115 & 0.0024 \\
\hline 32.9 & 80 & 5.5 & 0.004 & 0.0024 & 41 & 74.5 & 4.7 & 0.0091 & 0.0021 \\
\hline 32.9 & 80 & 6.7 & 0.0016 & 0.0001 & 41.11 & 73.85 & 4 & 0.0070 & 0.0006 \\
\hline 32.9 & 80 & 6.8 & 0.0015 & 0.0001 & 40.98 & 73.83 & 3.7 & 0.0064 & 0.0034 \\
\hline 32.9 & 80 & 6.9 & 0.0014 & 0.0003 & 40.8 & 74 & 4.8 & 0.0030 & 0.0020 \\
\hline 32.9 & 80 & 7.3 & 0.0011 & 0.0008 & 40.85 & 73.5 & $4.8^{*}$ & 0.0010 & 0.0003 \\
\hline 33.05 & 80.17 & $7.4^{*}$ & 0.0003 & 0.0003 & 40.85 & 73.5 & $5.4^{*}$ & 0.0007 & 0.0007 \\
\hline
\end{tabular}

* Maximum probable earthquake (MPE) from de-aggregation analysis 

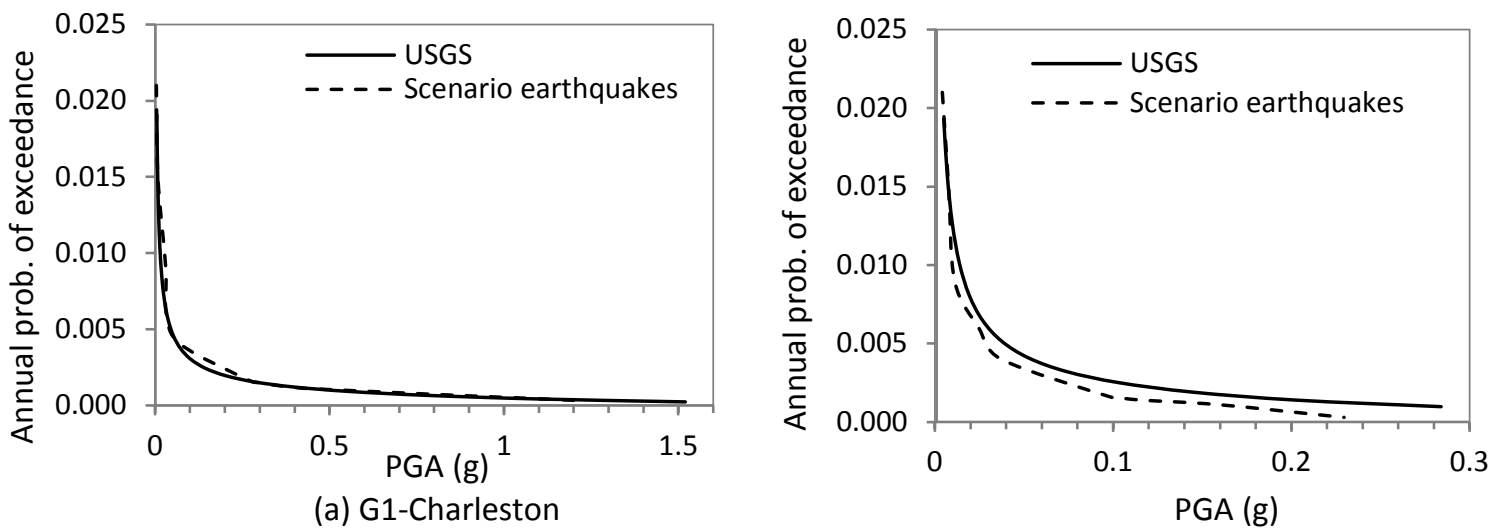

(a) G1-Charleston

(b) G5-Charleston

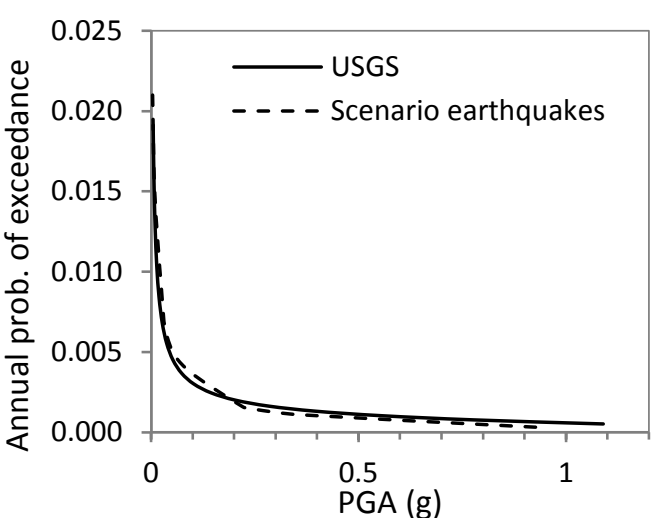

(c) G8-Charleston

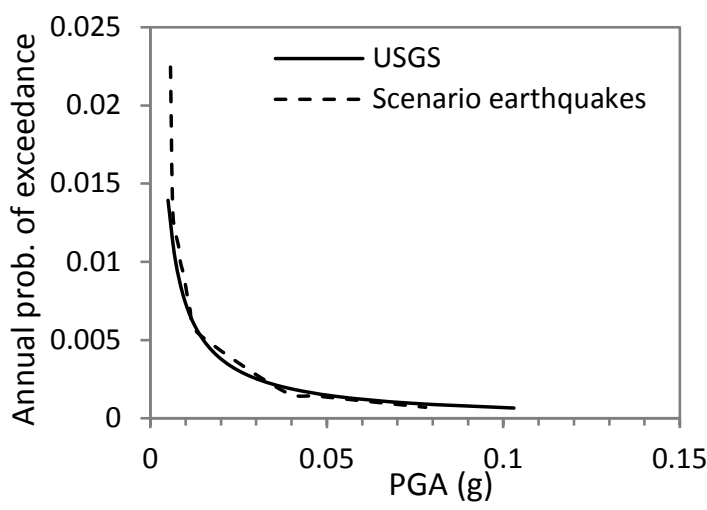

(e) G5-NY

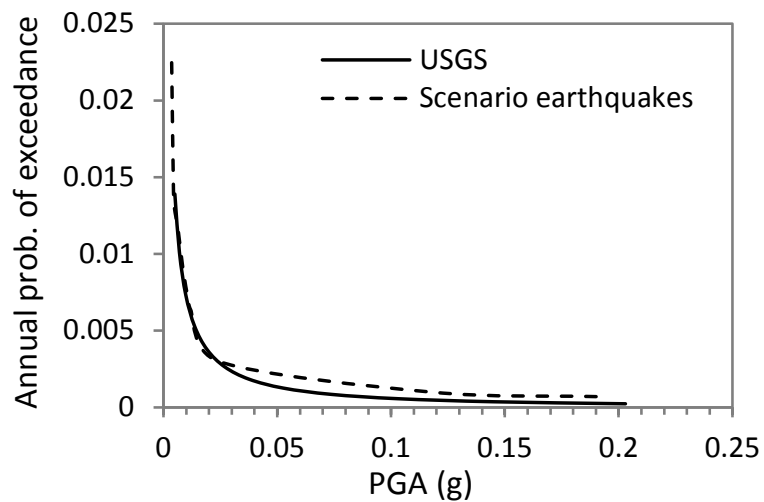

(d) G1-NY

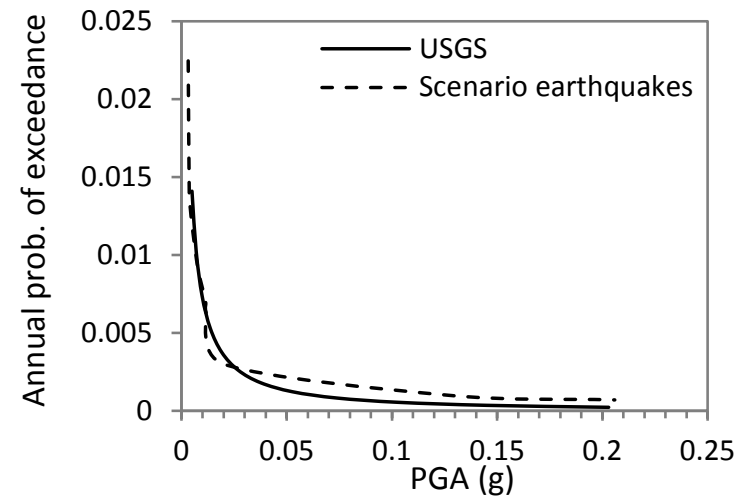

(f) G8-NY

Figure 8.1 Seismic hazard curves

(a) G1-Charleston; (b) G5-Charleston; (c) G8-Charleston; (d) G1-NY; (e) G5-NY; and (f) G8-NY 


\subsubsection{Hurricane Hazard Analysis}

For hurricane hazard, the hurricane simulation model used in Chapter 5 is also used here. The flow chart of the simulation model is shown again in Figure 8.2 for convenience. The required parameters for the hurricane simulation in South Carolina are taken from Huang et al. (2001a) and shown in Table 8.2. The parameters for New York City are found by fitting probability distributions to histograms of the parameters from Lin et al. (2010). It should be noted that while the simulation parameters for South Carolina from Huang et al. (2001a) are obtained from records of historical hurricanes, the parameters for New York from Lin et al. (2010) are based on simulated hurricanes due to lack of adequate historical data for New York.

Wind speed decay after landfall due to friction and reduction in storm's moisture for Charleston and New York is modeled using the model developed by Kaplan and DeMaria (1995) and Kaplan and DeMaria (2001), respectively. As the location of the power system is within approximately 50 miles of the coast in both locations, each hurricane is assumed to travel in a straight line from landfall to when it will pass through the study area. This assumption has been shown to be reasonable for areas within 50 miles of the coast (Brown, 2009). The simulation is carried out for 10,000 hurricane seasons. 


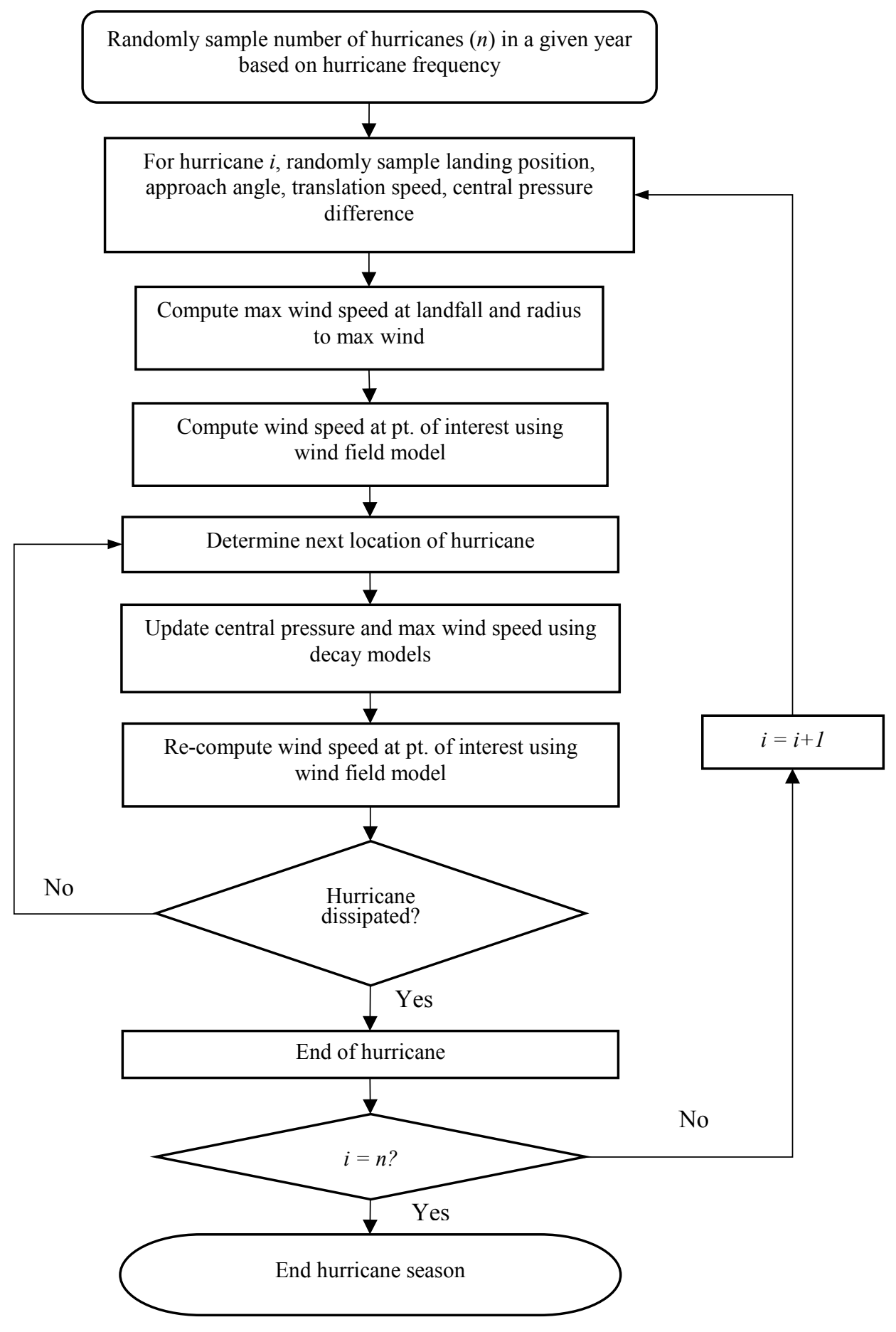

Figure 8.2 Hurricane simulation model flow chart 
Table 8.2 Statistics of hurricane simulation parameters

\begin{tabular}{llll}
\hline \hline \multirow{2}{*}{ Variable } & Distribution & \multicolumn{2}{l}{ Distribution parameters } \\
\cline { 3 - 4 } & & South Carolina & New York \\
\hline Annual frequency, $\lambda$ & Poisson & 0.306 & 0.26 \\
Approach angle, $\theta$ (degrees) & Normal/Uniform & $\mu=2.19$ & $0-75$ \\
& & $\sigma=42.77$ & \\
Central pressure difference & Weibull & $\mathrm{u}=51.12$ & $\mathrm{u}=32.34$ \\
& & $\mathrm{k}=3.155$ & $\mathrm{k}=2.85$ \\
Translation velocity & Lognormal & $\lambda=1.787$ & $\lambda=2.545$ \\
& & $\zeta=0.513$ & $\zeta=0.437$ \\
\hline \hline
\end{tabular}

For each hurricane, the maximum wind speeds at the middle of each transmission line are recorded as the hurricane passes through the study region. The maximum wind speed at the location of G1 is also recorded based on which the annual probability of exceedance is assigned to each recorded wind speed so as to match the resulting hazard curve with that obtained from ASCE 7-10 model which can be accessed from ATC (2015) for any location. Figure 8.3 shows a plot of the hazard curves at the location of G1 using wind speeds from ATC (2015) and from the hurricane simulation model described.

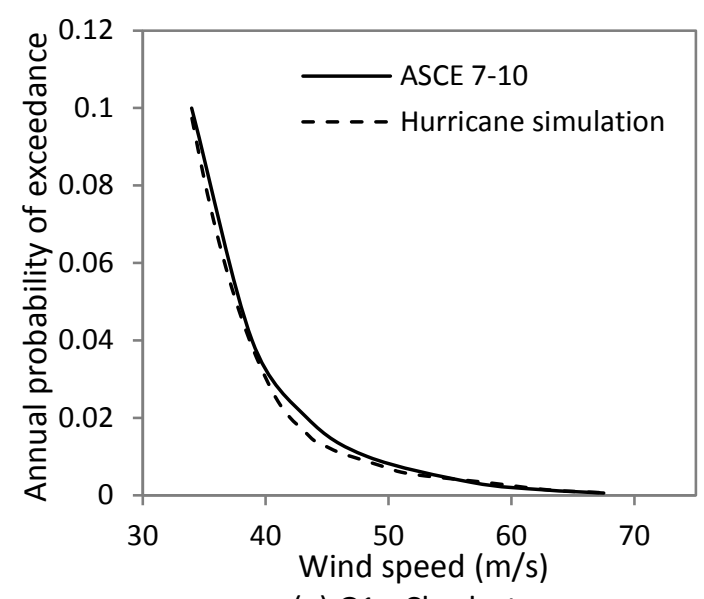

(a) G1 - Charleston

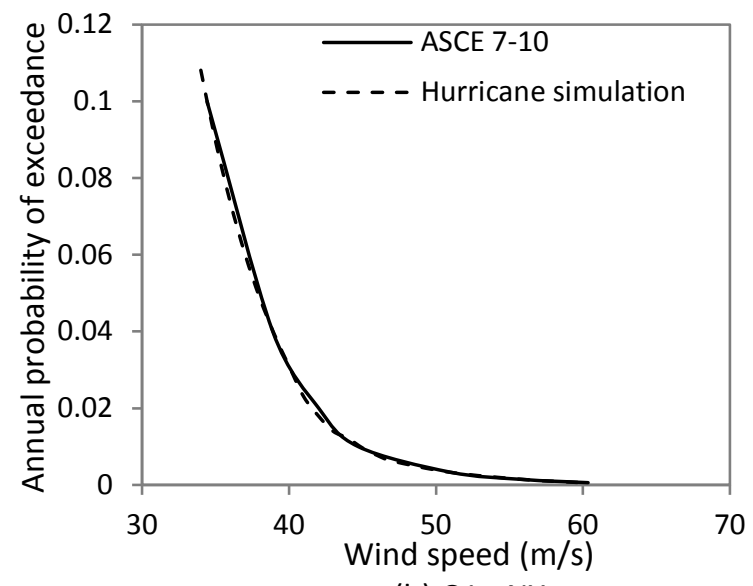

(b) G1 - NY

Figure 8.3 Hurricane hazard curves

(a) G1 - Charleston (b) G1 - NY 
Using the probabilistically weighted deterministic hazard scenarios approach, the annual probability of failure of any system component can be calculated using Equation (8.1).

$$
P_{A}=\sum_{i=1}^{n} \operatorname{Pr}(F \mid \text { Event } i) \cdot \operatorname{Pr}(\text { Event } i)
$$

where $P_{A}$ is the annual probability of failure of a component; $\operatorname{Pr}(F \mid$ Event $i)$ is the probability of failure of the component given the occurrence of hazard event $i ; \operatorname{Pr}($ Event $i)$ is the annual probability of occurrence of hazard event $i$; and $n$ is the total number of hazard events. Note that Equation (8.1) assumes independence between hazard events occurrence.

\subsection{Component Vulnerability}

Fragilities of substations and transmission structures discussed in the previous chapter are used here. However, in the original system, the substations are assumed to have standard (unanchored components). The fragility parameters of the three classes of substations are shown in Table 8.3 (FEMA, 2010). All four damage states will be considered in the cost analysis section. However, for the purpose of demonstrating other parts of the framework, it is assumed that substations in extensive damage state will lose their functionality and are considered failed (Dueñas-Osorio et al., 2007). 
Table 8.3 Lognormal parameters for seismic fragility of substations with standard components

\begin{tabular}{llll}
\hline \hline Substation & Damage State & Median PGA $(\mathrm{g})$ & Dispersion \\
Classification & & & \\
\hline \multirow{3}{*}{ Low voltage } & Slight/minor & 0.13 & 0.65 \\
& Exterate & 0.26 & 0.50 \\
& Complete & 0.34 & 0.40 \\
& Slight/minor & 0.74 & 0.40 \\
Medium voltage & Moderate & 0.20 & 0.60 \\
& Extensive & 0.30 & 0.50 \\
& Complete & 0.50 & 0.40 \\
High voltage & Slight $/$ minor & 0.09 & 0.40 \\
& Moderate & 0.13 & 0.50 \\
& Extensive & 0.17 & 0.40 \\
& Complete & 0.38 & 0.35 \\
\hline
\end{tabular}

\subsection{Multi-Hazard Risk Assessment}

As explained in the previous chapter, multi-hazard risk assessment of independent nonconcurrent hazards such as earthquakes and hurricanes is usually carried out through comparative approach using a common index. This is feasible because risk is not measured in hazard-specific units but in damage or loss-specific units such as damage to properties or disruption to economic activities (Kappes et al., 2012). The common risk indicator adopted here is system reliability (or unreliability). This allows direct quantitative comparison of the risks for the range of return periods (or annual probabilities of exceedance) covered by both hazards. Figure 8.4 shows the multi-hazard risk curves for the power system in the 2 locations for selected hazard levels. 


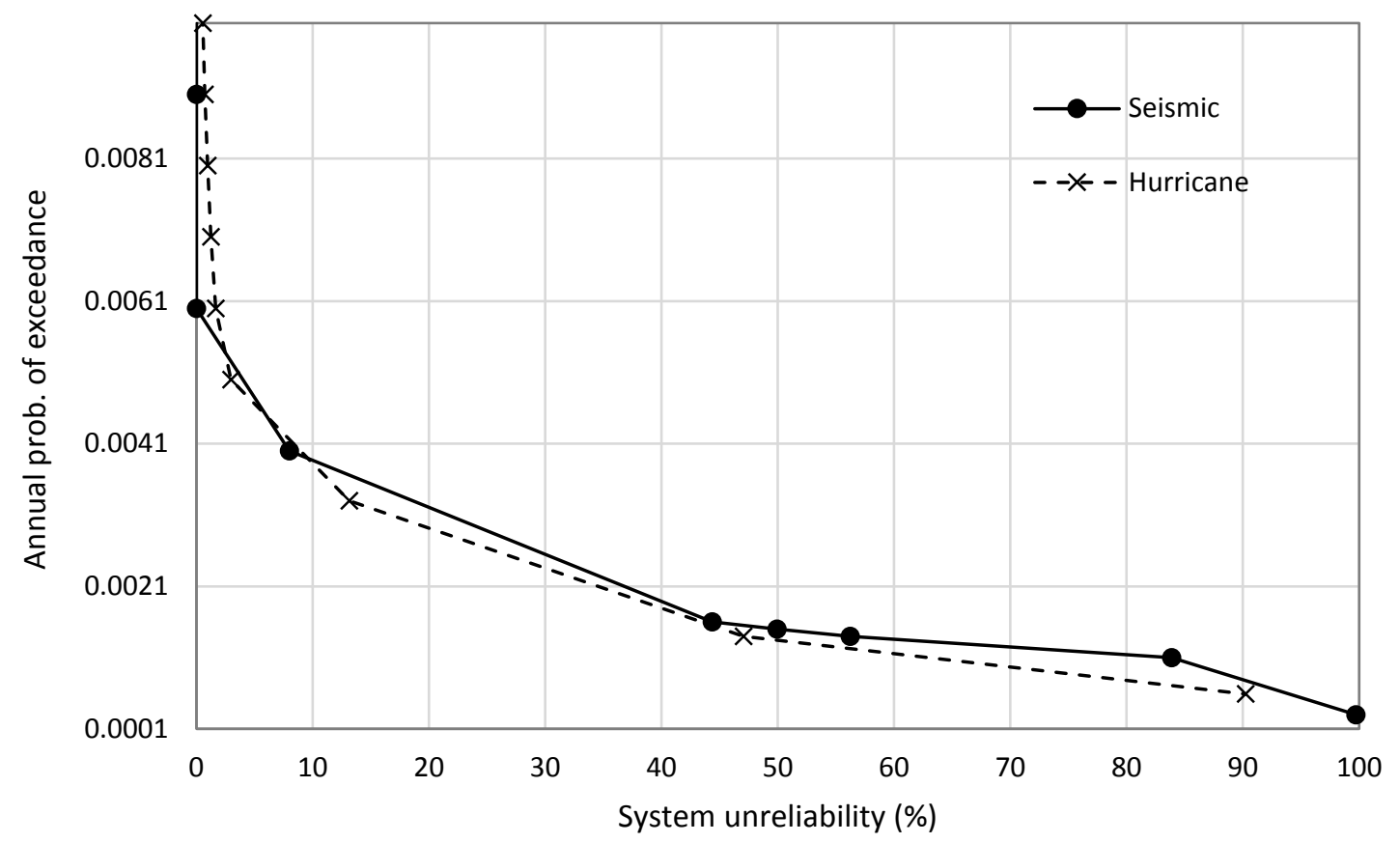

(a)

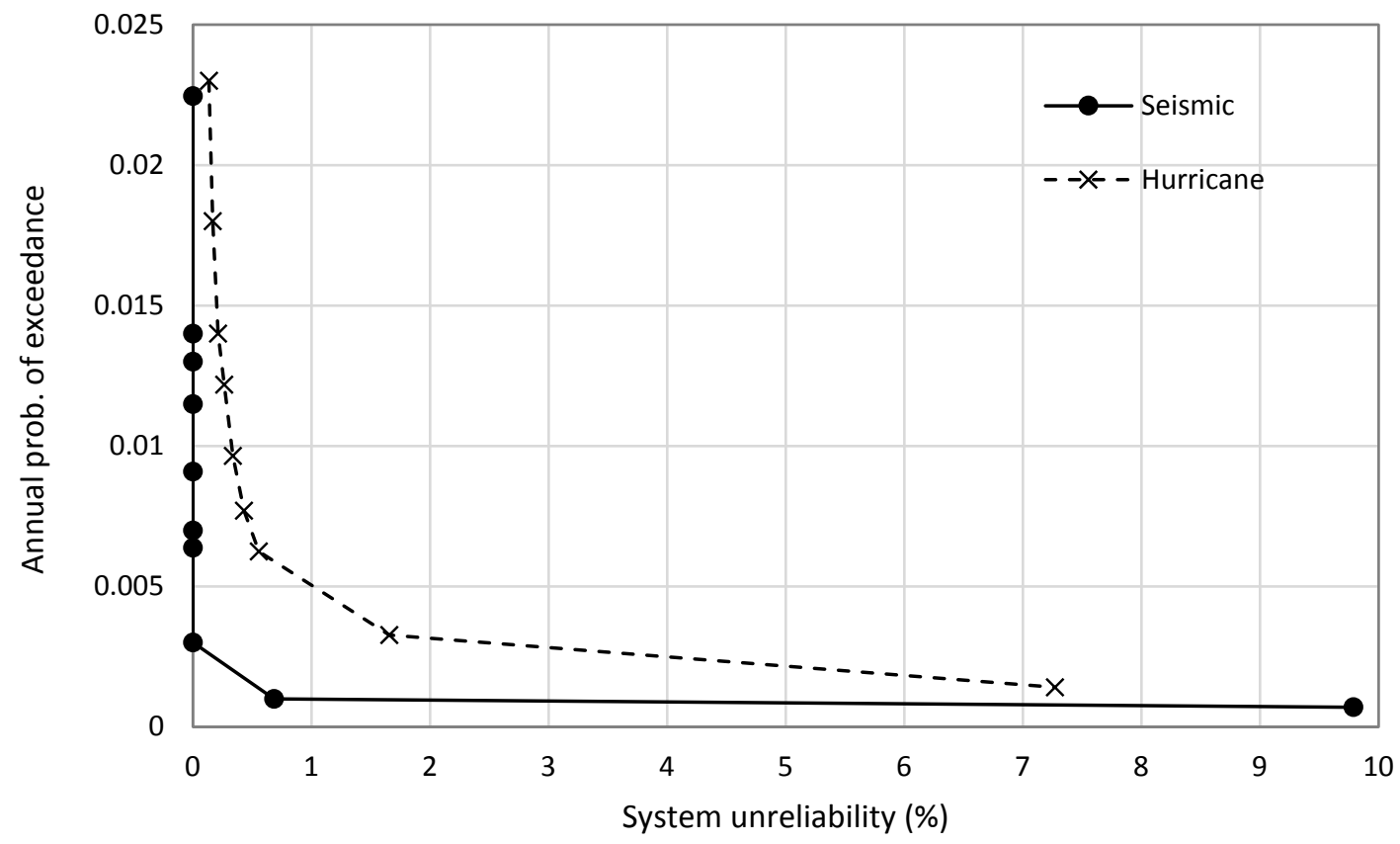

(b)

Figure 8.4 Multi-hazard risk curves

(a) Charleston, SC (b) New York, NY 
It can be seen from Figure 8.4(a) that at lower annual exceedance probabilities in Charleston, the risk due to seismic hazard is higher than that from hurricane hazard. For example, the annual probabilities of exceeding a system unreliability of $80 \%$ are about 0.0012 and 0.0008 for seismic and hurricane hazards, respectively. At higher annual exceedance probabilities, the risk due to hurricane hazard is higher. In other words, there is a higher probability that seismic hazard will cause major disruption to the system than hurricane hazard while there is a higher probability that hurricanes will cause minor disruptions to the system.

In the case of New York, it can be seen from Figure 8.4(b) that risk due to hurricane hazard is higher for the entire range of data plotted. In general, it can be seen that risk to the system due to both hurricane and seismic hazards is higher in Charleston than New York. For example, the annual probabilities of exceeding a system unreliability of $6 \%$ due to hurricane hazard are about 0.0045 and 0.0017 for Charleston and New York, respectively. Similarly, the probabilities due to the seismic hazard are 0.0045 and 0.0009 for Charleston and New York, respectively.

Even though hurricanes and earthquakes are independent non-concurrent hazards, annualizing the risk (measured as system unreliability in this case) can make the summation of risk due to both hazards feasible. Risk can be annualized by weighing each hazard level with its annual probability of occurrence. This is important when it comes to evaluating the cost-effectiveness of mitigation strategies as will be discussed later on.

For the sake of comparison, the probabilistic method used to plot the multi-hazard risk curves in Figure 7.11 in the previous chapter is used to plot the curves for the range of return periods in Figure 8.4. The comparison for Charleston is shown in Figure 8.5. It can be seen that risk curves from the two methods are similar. Relatively, the probabilistically weighted deterministic scenarios approach overestimates the risk in some sections of the curves. The comparison for New York is shown in Figure 8.6 where the probabilistically 
weighted deterministic scenarios approach results in higher risk than the probabilistic analysis.

The advantage of the probabilistic analysis used in the previous chapter is that it considers the entire range of hazard levels. However, the hazard levels corresponding to different return period at different locations does not necessarily occur at the same time or during the same hazard event. This is why the spatial variation of hazard intensity is lost in the aggregation process. The probabilistically weighted deterministic scenarios approach on the other hand models the spatial variation of hazard intensity during each event. However, due to the limitation on computational effort, the number of hazard events that can be considered is limited. Consequently, the approach might not cover the entire range of possible hazard levels. 


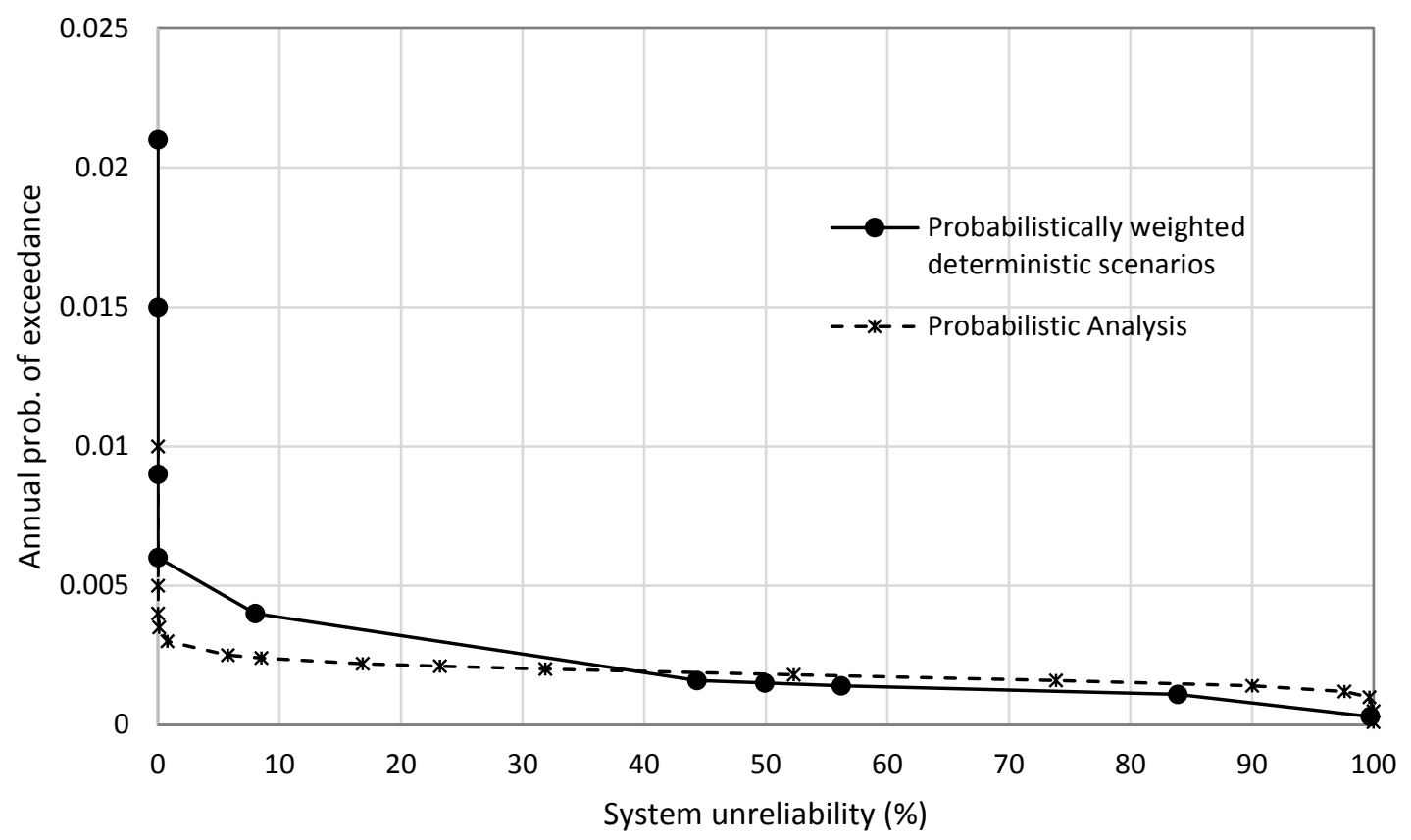

(a)

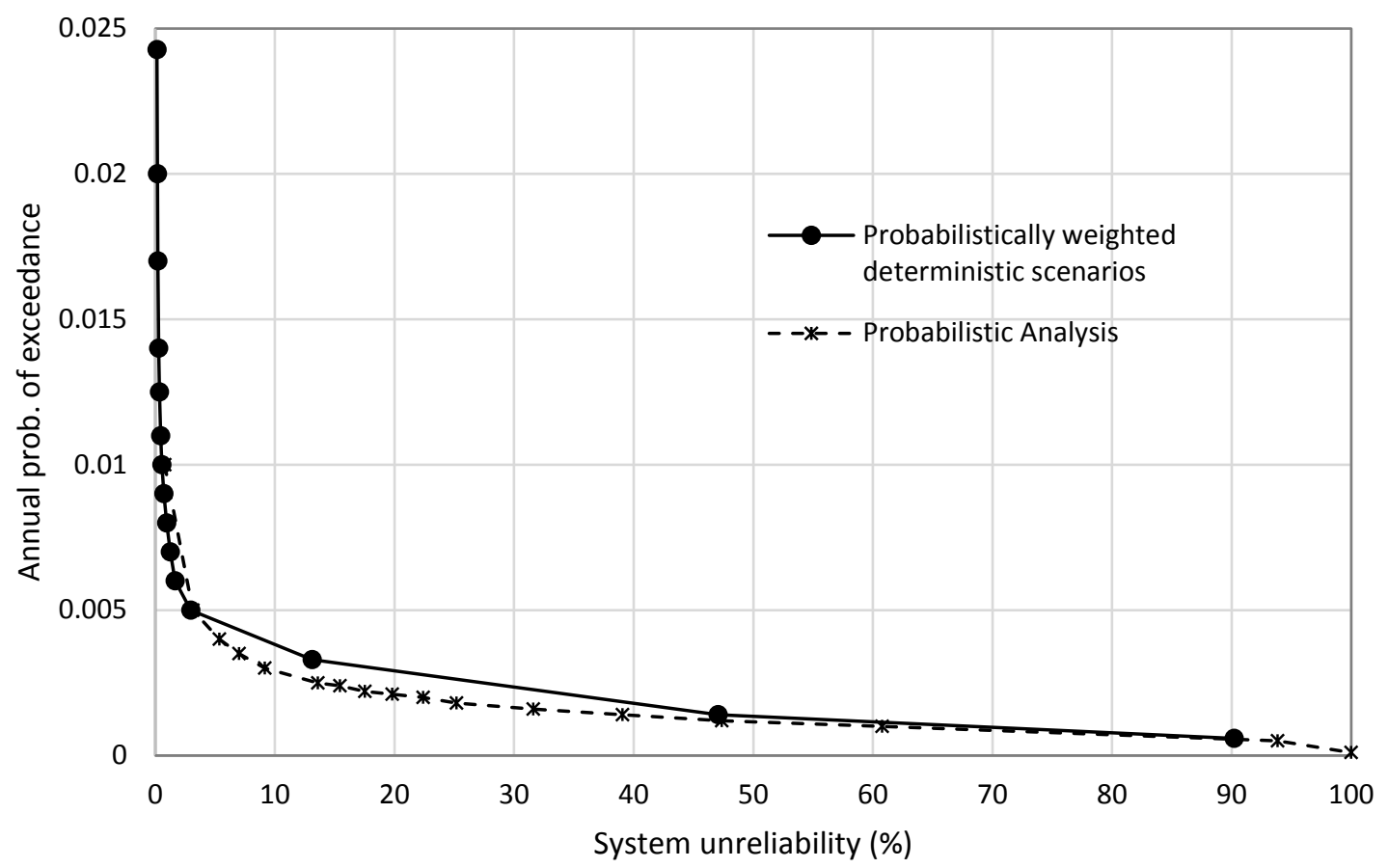

(b)

Figure 8.5 Comparison between probabilistically weighted deterministic scenarios method and probabilistic method for Charleston

(a) Seismic curves (b) Hurricane curves 


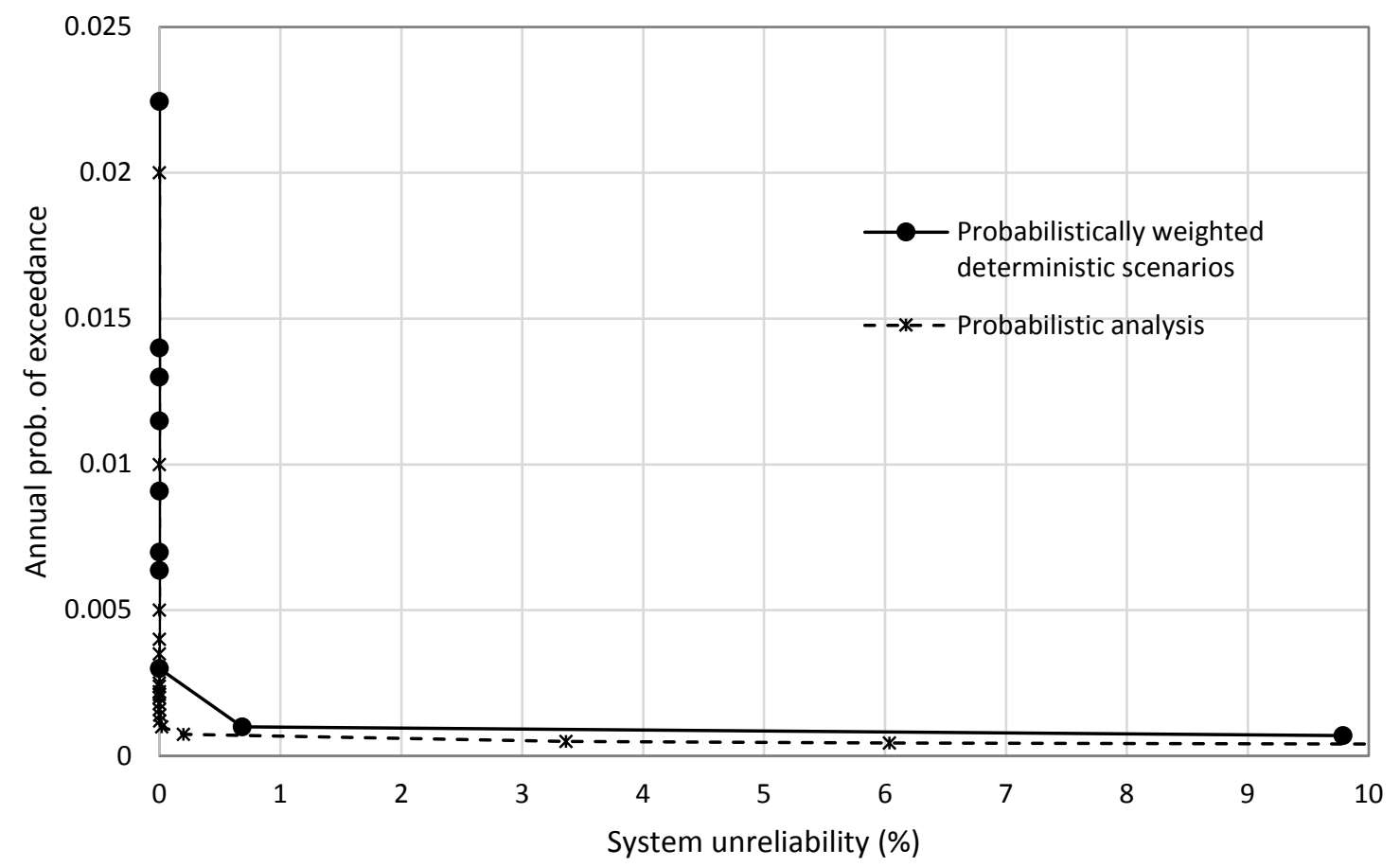

(a)

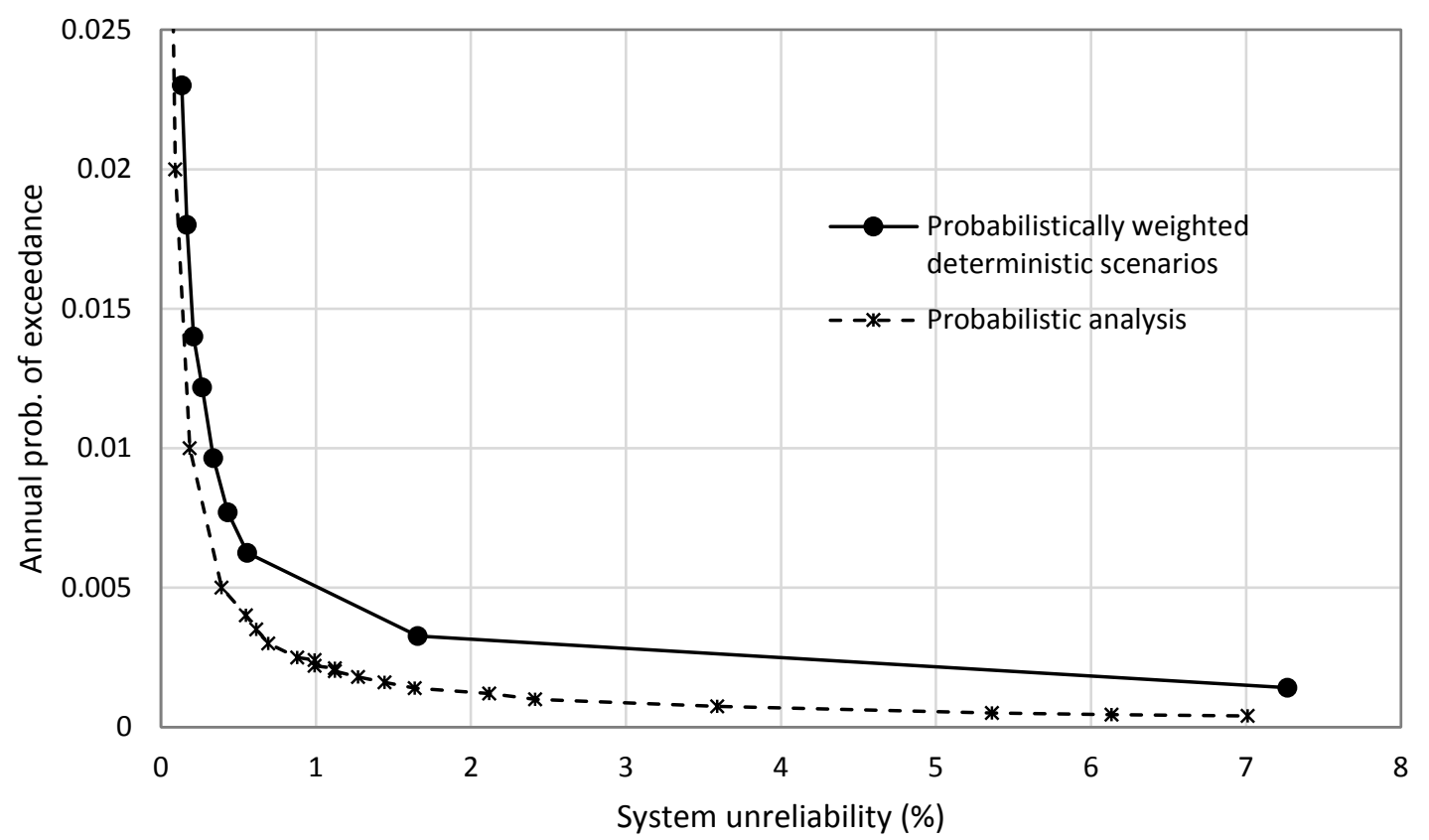

(b)

Figure 8.6 Comparison between probabilistically weighted deterministic scenarios method and probabilistic method for New York

(a) Seismic curves (b) Hurricane curves 


\subsection{Component Importance Measure}

Mitigation strategies for distributed infrastructure systems such as electric power systems invariably involve strengthening system components (substations and transmission line support structures in this case). Strengthening an entire system may, however, not be costeffective as some components have a greater effect on overall system reliability than others. Decisions on investment in mitigation strategies can be better made if the impact of strengthening different components on system reliability can be quantified. This can be achieved using some form of a measure of component importance.

One of the most widely used measures of component importance is Risk Achievement Worth (RAW). RAW is an indicator of two measures, (i) the 'worth' of a component in achieving the current level of system reliability, and (ii) the importance of maintaining or improving the current reliability of a component. RAW of each component, $i$, is given by Equation (8.2) (Rausand \& Høyland, 2004).

$$
R A W(i)=\frac{1-R_{S}\left(Q_{i}=1\right)}{1-R_{S}} \quad \text { for } i=1,2, \ldots, n
$$

where $R_{S}\left(Q_{i}=1\right)$ is the system reliability when component $i$ has failed while $R_{S}$ is the reliability of the original system. The risk achievement worth of all the substations and transmission lines in the system is presented in Figure 8.7 for the system in Charleston using annual probabilities of component failures. 


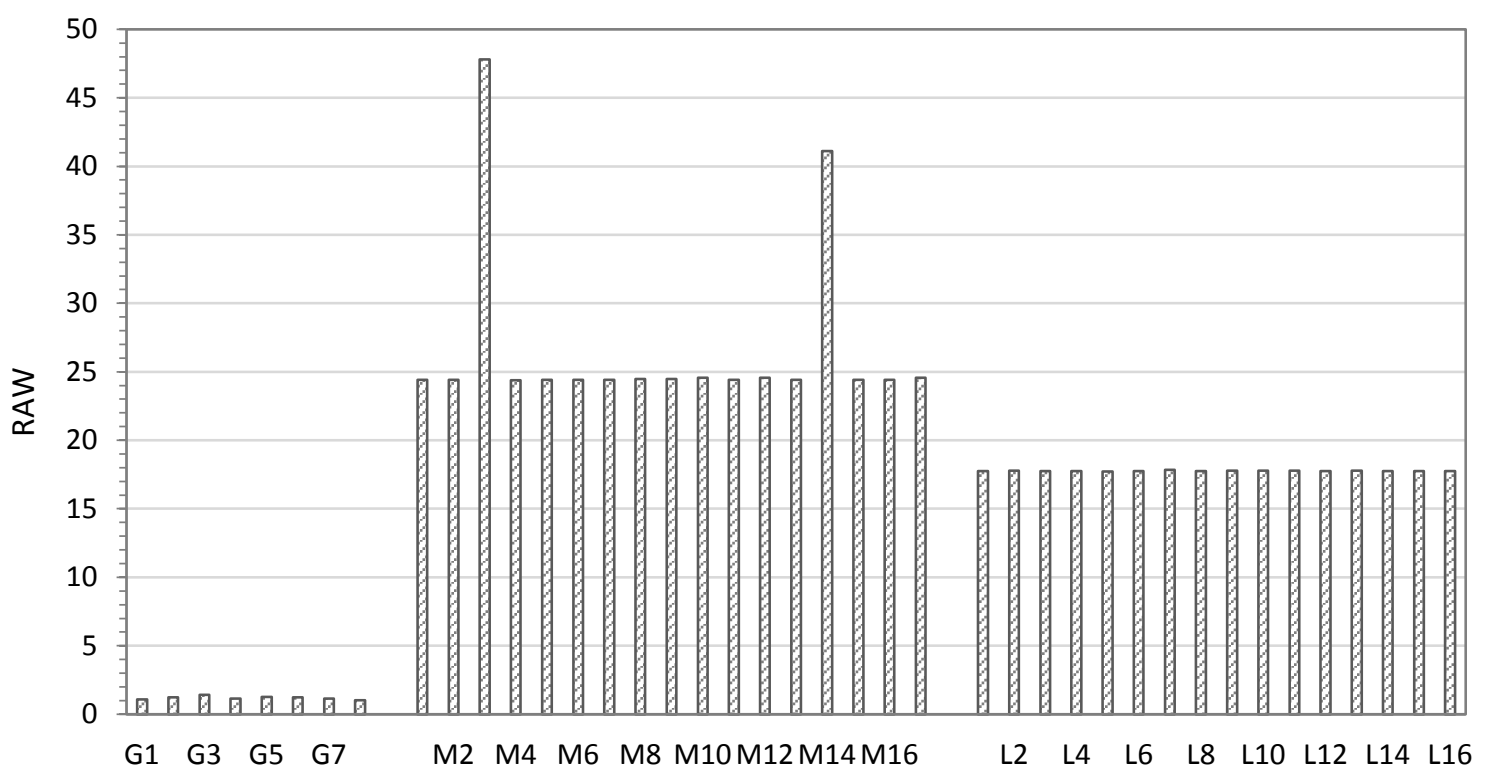

Substation

(a)

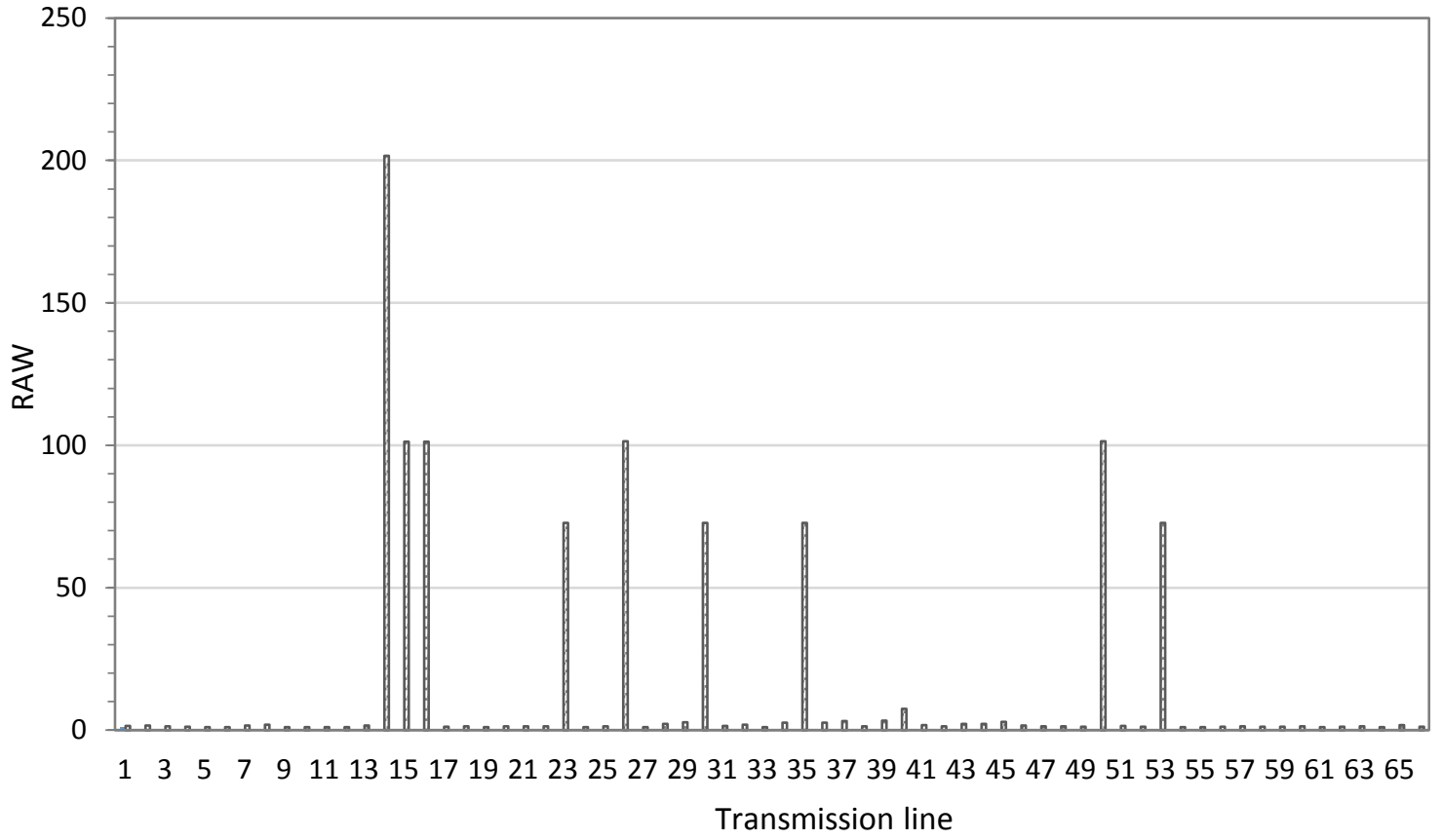

(b)

Figure 8.7 Risk achievement worth of components in Charleston

(a) Substations (b) Transmission lines 
It can be seen from Figure 8.7(a) that the RAW of the substations in each class is very similar. All gate stations (G1 - G8) have RAW of about 1.2, medium voltage substations (M1 - M17) have RAW of about 24.4 (with two exceptions), and low voltage substations (L1 - L16) have RAW of about 17.8. The lack of variation in RAW for each substation class is due to the redundancy in the system. For example, the gate stations should have had the largest RAW considering the fact that they are the supply stations, however, there is always more than one gate station supplying power to each demand substation. Consequently, failure of any one gate station will have a minimal impact on system reliability.

Looking at the demand substations (low and medium voltage), their RAW is very similar for each class because there is always an alternative path for power delivery from gate stations to demand stations even if a substation along a certain path of power delivery fails. Hence, failure of any demand substation will only cut-off power to the customers directly connected to it. The only exception is M3 and M14. This is because looking at Figure 7.2, it can be seen that power delivery to M4 depends on the reliability of M3 because there is no alternative path from any gate station to M4. Hence, failure of M3 will not only cut-off power to customers directly connected to it, but also to customers connected to M4. Similarly, power delivery to L5 depends on M14. A similar observation can be made from Figure 8.7(b) for transmission lines where lines such as 12 and 26 have higher RAW because of lack of redundancy to the substations they serve.

In general, it can be seen that RAW (and other similar component importance measures that consider the failure of single elements such as Risk Reduction Worth (RRW)) is not an effective measure of component importance in a complex system with high redundancy such as the power system considered in this case. This is because failure or removal of a single component will not significantly impact system reliability because the failure of the system or a significant part of the system is caused by combinations of failure of several components which make up the various minimal cut sets of the system. 
A better measure of component importance for redundant systems such as the power system in this study should take into account the number of minimal cuts that a component affects. The Fussel-Vesely importance measure takes this into account by defining component importance as a ratio of the probability of failure of at least one minimal cut containing the component in question at time $t$ to the probability that the system is failed at time $t$ (Fussell, 1975). In other words, the component contributes to system failure when a minimal cut set containing the component failed. This is, however, applicable to binary systems that can be defined as either functioning or not. That is, it applies to systems where the failure of at least one minimal cut set leads to overall system failure. In the case of electric power networks with several load points, failure of power delivery to one substation or one minimal cut set does not imply system failure. This implies that the system has several functional states.

To overcome the above shortcomings, the following component importance index is proposed:

$$
I_{i}=\frac{1-R_{S_{-} a v}}{1-R_{S}} \quad \text { for } i=1,2, \ldots, n
$$

where $I_{i}$ is the importance index of component $i ; R_{S_{-} a v}$ is the average system reliability among all scenarios where a minimal cut set containing component $i$ is failed; and $R_{S}$ is the reliability of the original system. The proposed component importance index models multi-element failure which is required to bring about overall system failure in a networked system. This is important as natural hazards such as earthquakes usually results in the failure of multiple elements at a time. Hence, the proposed index models the likelihood that a combination of failed elements that contain the element in question contributes to overall system failure. $R_{S_{-} a v}$ takes into account the fact that a component can appear in several minimal cut sets that govern power delivery to several subsystems or substations. Figure 8.8(a) \& Figure 8.8(b) show the importance index of substations and transmission lines in the system, respectively, using the proposed measure. 


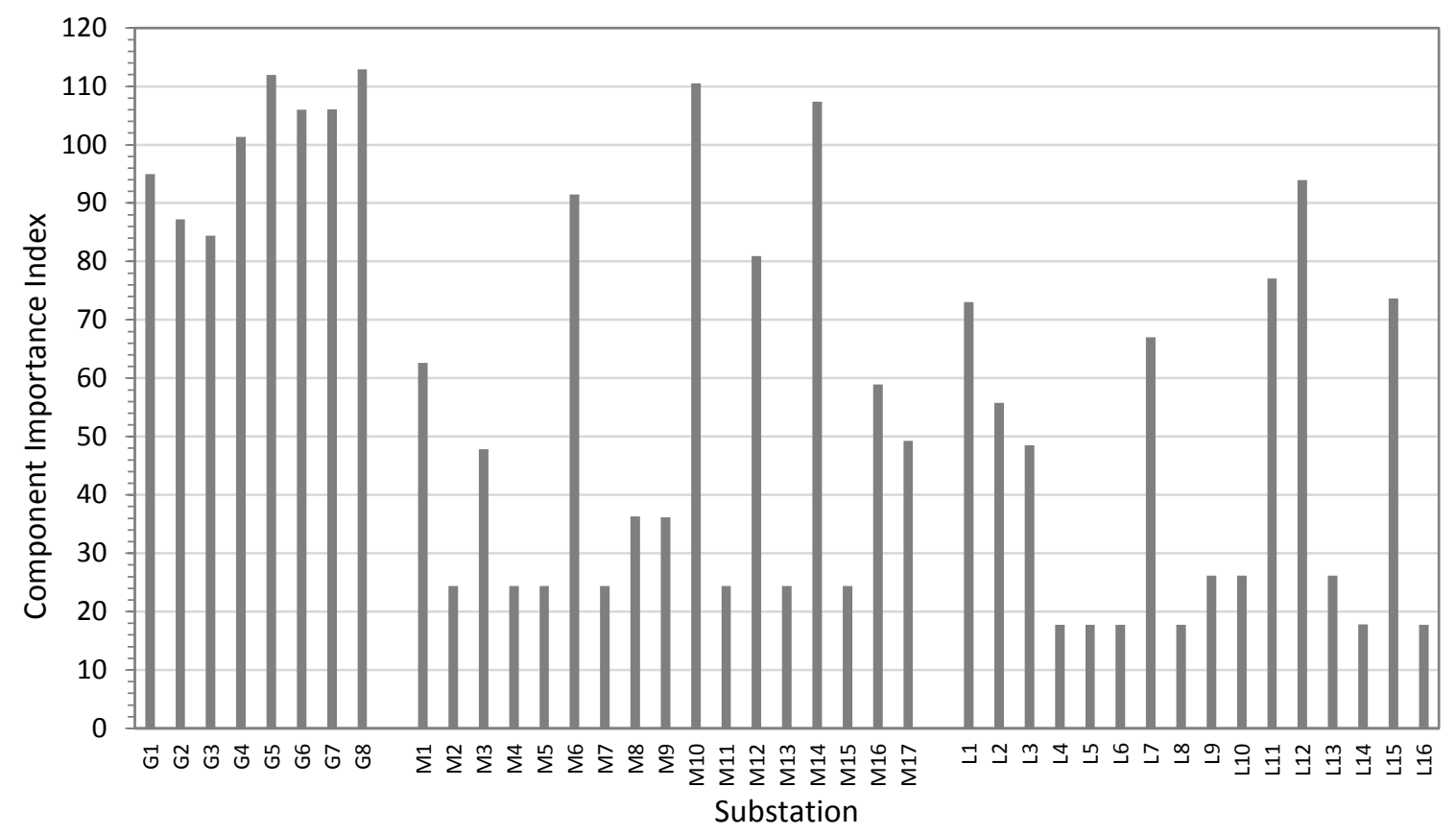

(a)

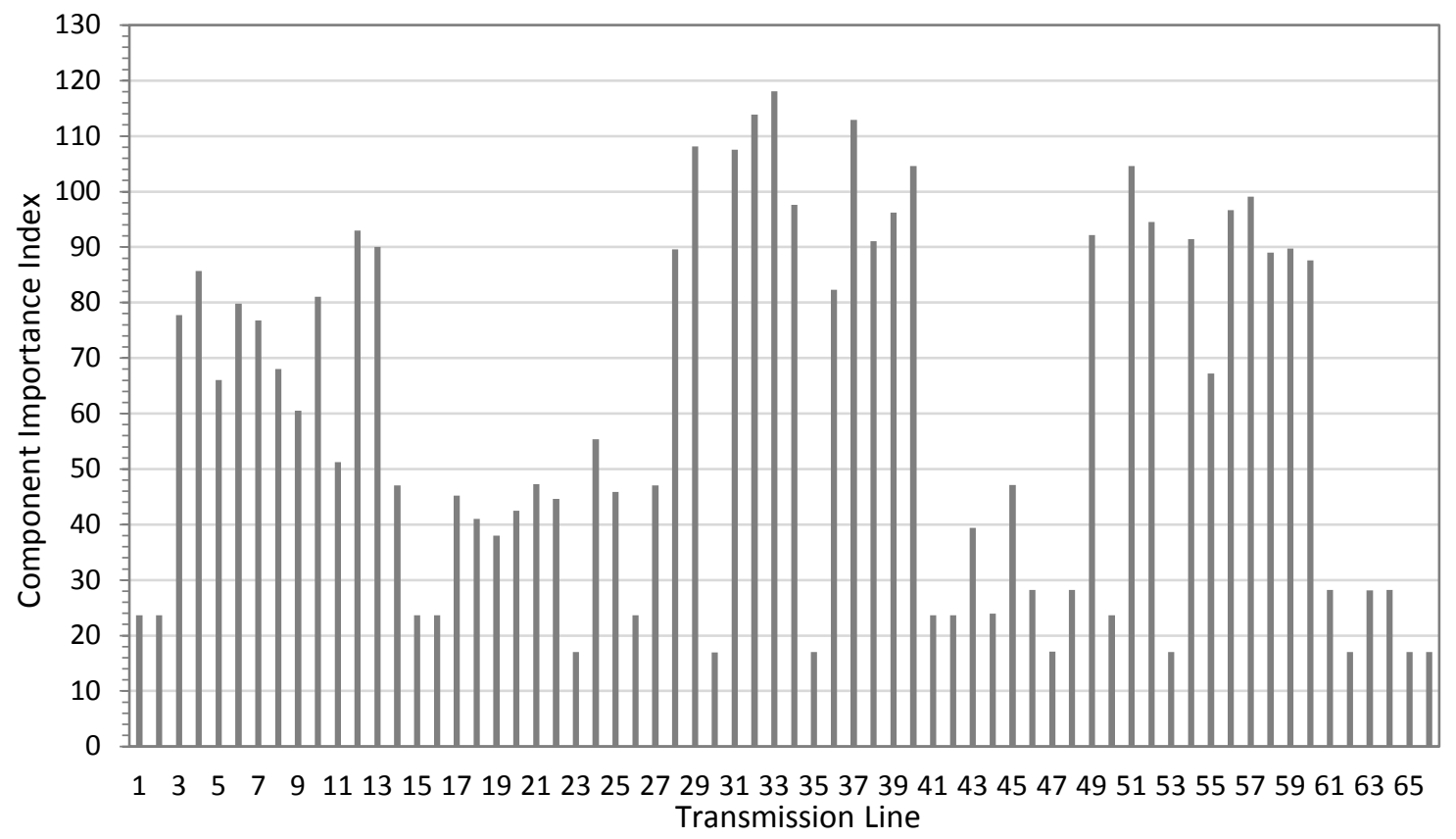

(b)

Figure 8.8 Importance index of components in Charleston

(a) Substations (b) Transmission lines 
From Figure 8.8(a), it can be seen that the substations with the lowest importance index ( $I_{i}$ $<20)$ are L4, L5, L6, L8, L14, and L16. This is because their failure does not affect power delivery to other substations. All the gate stations show relatively high importance index as they are the supply stations and failure of a combination of gate stations will cut power supply to several demand stations. Looking at Figure 8.8(b), it can be seen that transmission lines with $I_{i}<20$ are lines whose failure will not affect power delivery to more than one substation. Transmission lines such as lines 33 and 40 whose failure, especially in combination with other lines, will cut-off power supply to several substations have relatively higher $I_{i}$.

\subsection{Multi-Hazard Mitigation Strategies}

Seven mitigation strategies have been selected to demonstrate the proposed framework, based on the result of component importance measure above. These are:

1. Strengthening substations with $I_{i}>100(7$ substations $=17 \%)$

2. Strengthening substations with $I_{i}>70(16$ substations $=39 \%)$

3. Strengthening transmission lines with $I_{i}>100(7$ lines $=11 \%)$

4. Strengthening transmission lines with $I_{i}>80(24$ lines $=36 \%)$

5. Strengthening substations and transmission lines with $I_{i}>100$

6. Strengthening substations with $I_{i}>70$ and transmission lines with $I_{i}>80$

7. Strengthening entire system (substations and transmission lines)

The first 6 strategies are selected to represent different levels of targeted hardening of the system. The last mitigation strategy is chosen to investigate the effectiveness of targeted hardening as opposed to hardening the entire system. 
Strengthening substations involve substituting standard components with anchored/seismic components. While the current requirement for substation component design called for anchored components, a lot of existing substations are composed of standard unanchored components (Knight \& Kempner Jr, 2009a). The fragility of anchored substations is taken from FEMA (2005) in which it is modeled with lognormal distribution with parameters given in Table 8.4 .

Table 8.4 Lognormal parameters for seismic fragility of substations with anchored components

\begin{tabular}{llll}
\hline \hline Substation & Damage State & Median PGA $(\mathrm{g})$ & Dispersion \\
Classification & Slight/minor & 0.15 & 0.70 \\
& Moderate & 0.29 & 0.55 \\
Low voltage & Extensive & 0.45 & 0.45 \\
& Complete & 0.90 & 0.45 \\
\hline \multirow{3}{*}{ Medium voltage } & Slight/minor & 0.15 & 0.60 \\
& Moderate & 0.25 & 0.50 \\
& Extensive & 0.35 & 0.40 \\
& Complete & 0.70 & 0.40 \\
\hline \multirow{3}{*}{ High voltage } & Slight $/$ minor & 0.11 & 0.50 \\
& Moderate & 0.15 & 0.45 \\
& Extensive & 0.20 & 0.35 \\
& Complete & 0.47 & 0.40 \\
\hline
\end{tabular}

Strengthening transmission lines involve strengthening existing transmission line support structures to conform with the current National Electrical Safety Code (NESC) standard which requires all transmission structures over $60 \mathrm{ft}$. to be designed for extreme wind and ice loadings (IEEE, 2012). The fragility function for upgraded transmission structures 
developed based on damage data from utility companies is given by Equation (8.4) (Brown, 2009).

$$
P_{S}=\min \left\{\left[\left(2 \cdot 10^{-8}\right) e^{0.0834 \cdot v}\right], 1\right\}
$$

where $P_{S}$ is the probability of failure of a transmission support structure at a given wind speed, $v$.

The fragility curves of existing transmission structures using Equation ((7.5) and upgraded transmission structures using Equation ((8.4) are plotted in Figure 8.9.

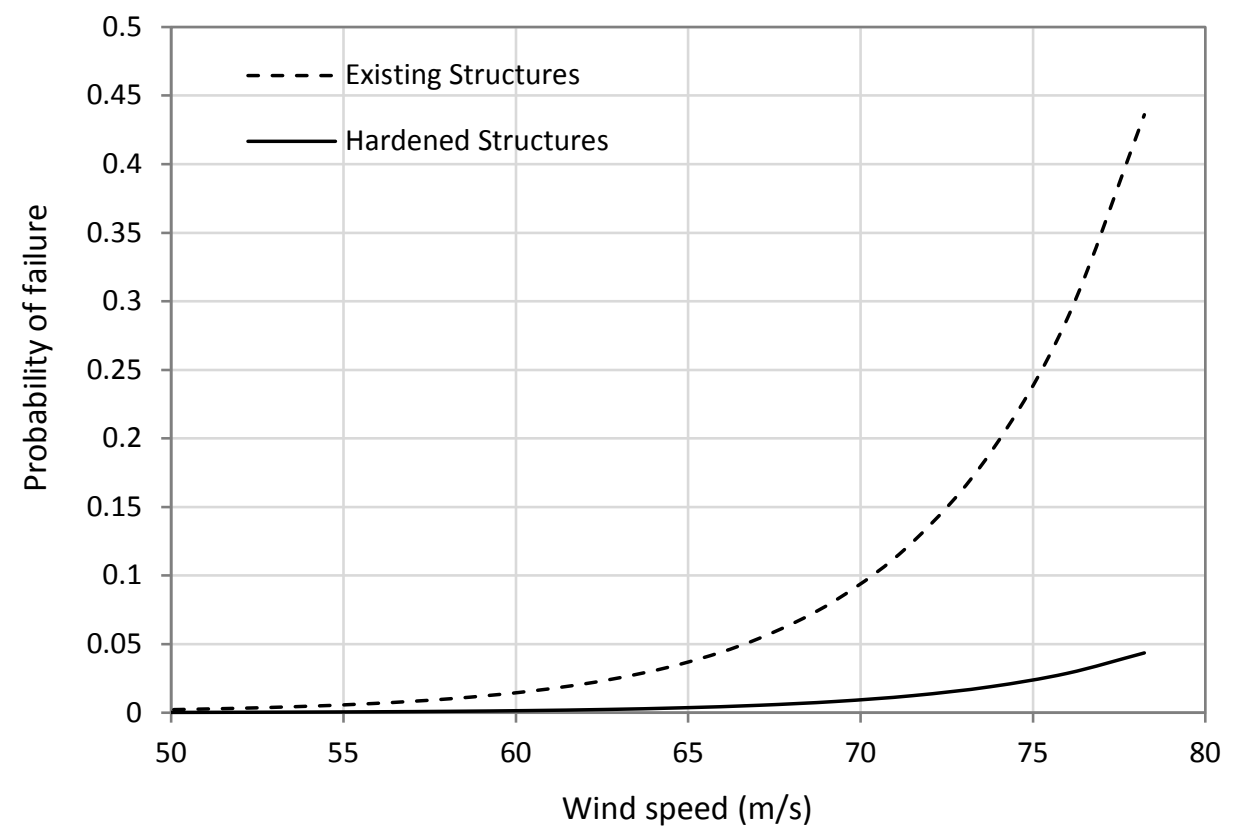

Figure 8.9 Fragilities curves of existing and hardened transmission structures 


\subsection{Evaluation of Cost-Effectiveness through Life-cycle Cost Analysis}

To investigate the effectiveness of mitigation strategies and prioritize investment, a decision-supporting tool is required to estimate costs and benefits, as well as the economic efficiency of mitigation policies. In this study, the net discounted benefit is used as a decision-support tool to estimate the benefit of the proposed mitigation strategies. The net discounted benefit is evaluated by calculating and discounting the costs and benefits arising over time, and the difference taken. The total costs and benefits are calculated through a life-cycle cost analysis over the remaining lifespan of the system. The benefit is the reduction in damages due to mitigation, while the cost is the cost of implementing the mitigation strategy. A fixed discount rate is used to convert costs over time to their equivalent present value. If the net discounted benefit is positive (benefits exceed costs), then the mitigation strategy is considered effective.

The various costs considered in the life cycle cost analysis are discussed below:

i. Mitigation cost: this is the cost of implementing a specific mitigation strategy which is assumed to be carried out in the first year of analysis.

ii. Maintenance cost: this is the cost of periodic maintenance performed by the utility company on the substation and transmission lines. The total maintenance cost for each component for the entire analysis period is given by Equation (8.5).

$$
C_{m}=\sum_{t} \sum_{i} m_{i}(t) \cdot(1+\lambda)^{-t}
$$


where $m_{i}$ is the annual maintenance cost for element $i$ at year $t$; and $\lambda$ is the discount rate.

iii. Repair cost: this is the cost of repairing damage to the system after an earthquake or hurricane. For damage to substations due to earthquakes, the annual repair cost for each substation is given by Equation (8.6) which is based on the theorem of total probability.

$$
C_{r S}=\sum_{t} \sum_{d=1}^{D} \sum_{i=1}^{n} \operatorname{Pr}(d \mid \text { Event } i) \cdot \operatorname{Pr}(\text { Event } i) \cdot C_{d} \cdot(1+\lambda)^{-t}
$$

where $C_{r s}$ is the annual repair cost per substation; $\operatorname{Pr}(d \mid$ Event $i)$ is the probability of occurrence of damage state $d$ given the occurrence of seismic event $i$; $\operatorname{Pr}($ Event $i)$ is the annual probability of occurrence of seismic event $i ; C_{d}$ is the cost of repairing the substation in damage state $d ; D$ is the total number of damage states; and $n$ is the total number of seismic events.

For damage to transmission lines after hurricanes, the repair cost is the cost of replacing failed transmission structures. Failed structures are replaced with new ones of the same class. The repair cost for a line for the entire lifespan of the system is given by Equation (8.7).

$$
C_{r L}=\sum_{t} \sum_{i=1}^{n} \operatorname{Pr}\left(F_{S} \mid \text { Event } i\right) \cdot \operatorname{Pr}(\text { Event } i) \cdot N \cdot C_{r} \cdot(1+\lambda)^{-t}
$$

where $C_{r L}$ is the repair cost of transmission line; $\operatorname{Pr}\left(F_{S} \mid\right.$ Event $\left.i\right)$ is the probability of failure of a structure given hurricane event $i ; \operatorname{Pr}($ Event $i)$ is the annual probability of occurrence of hurricane event $i ; N$ is the total number of structures in a line; and $C_{r}$ is the unit repair cost of structures in a line. Note that unlike substations, 
transmission structures and lines are modeled with only one damage state which is defined as failure.

iv. Revenue loss: this is the cost incurred by the utility company due to the interruption in power supply caused by earthquakes and hurricanes. It is a function of unmet demand, the time to restore the system after an event, and the unit cost of electricity. For failure of substations due to seismic hazard, the unmet demand for a substation is given by Equation (8.8).

$$
U_{D}=\int_{t=0}^{t_{r}} K_{i}(t) \cdot p(t) d t
$$

where $U_{D}$ is the unmet demand; $K_{i}(t)$ is the demand on substation $i$ at time $t ; p(t)$ is the unit price of electricity at time $t ; t_{r}$ is the time to repair the substation. The time to repair substations depends on the type and damage level of the substation. The revenue loss due to the failure of a substation is then given by Equation (8.9).

$$
C_{R s}=\sum_{t} \sum_{d=1}^{D} \sum_{i=1}^{n}\left(U_{D} \mid d \cdot \text { Event } i\right) \cdot \operatorname{Pr}(d \mid \text { Event } i) \cdot \operatorname{Pr}(\text { Event } i) \cdot(1+\lambda)^{-t}
$$

where $C_{R S}$ is the total revenue loss due to failure of substation; $\left(U_{D} \mid d \cdot\right.$ Event $\left.i\right)$ is the unmet demand given damage state $d$ and event $i ; \operatorname{Pr}(d \mid$ Event $i)$ is the probability of occurrence of damage state $d$ given the occurrence of seismic event $i$; $\operatorname{Pr}($ Event $i)$ is the annual probability of occurrence of seismic event $i$.

For transmission line failure due to hurricanes, failure of a single transmission line does not necessarily cut power to customers if there are other routes available for power to reach a particular substation. Hence, revenue loss due to hurricane hazard is calculated by considering the failure of all transmission lines supplying power to a substation. The unmet demand for a substation due to the failure of transmission lines 
is also calculated using Equation (8.8), where $t_{r}$ is the time to repair the transmission lines in this case. The revenue loss for a substation due to the failure of transmission lines is then calculated using Equation (8.10).

$C_{R L}=\sum_{t} \sum_{i=1}^{n}\left(U_{D} \mid F \cdot\right.$ Event $\left.i\right) \cdot \operatorname{Pr}(F \mid$ Event $i) \cdot \operatorname{Pr}($ Event $i) \cdot(1+\lambda)^{-t}$

where $C_{R L}$ is the annual revenue loss due to failure of transmission lines; $\left(U_{D} \mid F\right.$. Event $i)$ is the unmet demand given failure of all lines and event $i ; \operatorname{Pr}(F \mid$ Event $i)$ is the probability of failure of all transmission lines supplying power to a substation given the occurrence of hurricane event $i ; \operatorname{Pr}($ Event $i)$ is the annual probability of occurrence of hurricane event $i$.

$$
\operatorname{Pr}(F \mid \text { Event } i)=\operatorname{Pr}\left(L_{1} \cap L_{2} \cap \ldots \cap L_{k} \mid \text { Event } i\right)
$$

$\operatorname{Pr}\left(L_{1} \cap L_{2} \cap \ldots \cap L_{k}\right)$ is the probability of failure of all transmission lines supplying power to a substation, where the failures are assumed to be independent.

v. Societal economic loss: this is the direct economic loss to customers resulting from the interruption in power supply. For damage to substations due to earthquakes, the economic loss for a substation during a seismic event is given by Equation (8.12).

$$
E_{L}=\int_{t=0}^{t_{r}} L(t) d t
$$

where $L(t)$ is the monetary loss per hour; $t_{r}$ is repair time of the substation. The total societal economic loss for each substation is then given by Equation (8.13). 


$$
C_{E S}=\sum_{t} \sum_{d=1}^{D} \sum_{i=1}^{n}\left(E_{L} \mid d \cdot \text { Event } i\right) \cdot \operatorname{Pr}(d \mid \text { Event } i) \cdot \operatorname{Pr}(\text { Event } i) \cdot(1+\lambda)^{-t}
$$

where $C_{E S}$ is the total societal economic loss due to failure of substation; $\left(E_{L} \mid d\right.$. Event $i$ ) is the expected economic loss given damage state $d$ and event $i$; $\operatorname{Pr}(d \mid$ Event $i)$ is the probability of occurrence of damage state $d$ given the occurrence of seismic event $i ; \operatorname{Pr}($ Event $i)$ is the annual probability of occurrence of seismic event $i$.

As in the case of revenue loss, failure of one transmission line due to hurricane might not necessarily lead to a power outage and economic loss to customers. Hence, the failure of all lines connected to a substation is considered. The economic loss for a substation due to the failure of lines is given by Equation (8.14).

$C_{E L}=\sum_{t} \sum_{i=1}^{n}\left(E_{L} \mid F \cdot\right.$ Event $\left.i\right) \cdot \operatorname{Pr}(F \mid$ Event $i) \cdot \operatorname{Pr}($ Event $i) \cdot(1+\lambda)^{-t}$

where $C_{E L}$ is the annual societal loss due to failure of transmission lines; $\left(E_{L} \mid F\right.$. Event $i$ ) is the economic loss given failure of all lines and event $i ; \operatorname{Pr}(F \mid$ Event $i)$ is the probability of failure of all transmission lines supplying power to a substation given the occurrence of hurricane event $i ; \operatorname{Pr}(E v e n t i)$ is the annual probability of occurrence of hurricane event $i$.

The remaining service life of the system, i.e., the period of time over which the life cycle cost analysis is performed is assumed to be 50 years. Information regarding cost, time to repair failed elements, economic loss per customer, and average power consumptions are given in Table 8.5. It is acknowledged that the relevant data for cost analysis will vary from region to region and utility companies should use their own data when performing the analysis. The data in Table 8.5 is adopted to demonstrate the proposed framework. Low voltage substations are assumed to contain one 25 MVA transformer, medium voltage 
substations are assumed to contain two 25 MVA transformers, while high voltage substation are assumed to contain four 75 MVA transformers. It is also assumed that $1 \%$ of all customers served are commercial customers, $0.01 \%$ are industrial customers while the rest are residential customers. 
Table 8.5 Life cycle cost analysis parameters

\begin{tabular}{|c|c|c|}
\hline Parameters & Value & Source \\
\hline Mitigation cost for transmission lines & $\$ 60,000 /$ structure & Brown (2009) \\
\hline $\begin{array}{l}\text { Cost of transmission structure replacement } \\
\text { under storm condition }\end{array}$ & $\$ 120,000 /$ structure & Brown (2009) \\
\hline Annual maintenance cost (transmission lines) & $\$ 40 /$ mile & Brown (2009) \\
\hline Unit price of electricity, $p$ & $\$ 0.11 / \mathrm{kWh}$ & $\mathrm{Xu}$ and Brown (2008b) \\
\hline Economic loss (residential) & $\$ 2.70 / \mathrm{h}$ & LaCommare and Eto (2006) \\
\hline Economic loss (commercial) & $\$ 886 / \mathrm{h}$ & LaCommare and Eto (2006) \\
\hline Economic loss (industrial) & $\$ 3,253 / \mathrm{h}$ & LaCommare and Eto (2006) \\
\hline Average consumption (residential) & $1.5 \mathrm{~kW} / \mathrm{h}$ & EIA (2013) \\
\hline Average consumption (commercial) & $10.1 \mathrm{~kW} / \mathrm{h}$ & EIA (2013) \\
\hline Average consumption (industrial) & $39.4 \mathrm{~kW} / \mathrm{h}$ & EIA (2013) \\
\hline Transmission line repair time & 3 days & Romero et al. (2015) \\
\hline Substation repair time (minor/slight damage) & 1 day & FEMA (2010) \\
\hline Substation repair time (moderate damage) & 3 days & FEMA (2010) \\
\hline Substation repair time (extensive damage) & 7 days & FEMA (2010) \\
\hline Substation repair time (complete damage) & 30 days & FEMA (2010) \\
\hline Cost of low voltage substation & $\$ 2.8$ million & Balducci et al. (2006) \\
\hline Cost of medium voltage substation & $\$ 5.6$ million & Balducci et al. (2006) \\
\hline Cost of high voltage substation & $\$ 33.7$ million & Balducci et al. (2006) \\
\hline Mitigation cost for substations & $2 \%$ of cost & Assumed \\
\hline $\begin{array}{l}\text { Substation repair cost (damage state: } \\
\text { slight/minor) }\end{array}$ & $5 \%$ of cost & $\begin{array}{l}\text { Assumed based on definition of } \\
\text { damage states from FEMA } \\
(2010)\end{array}$ \\
\hline Substation repair cost (damage state: moderate) & $40 \%$ of cost & Same as above \\
\hline Substation repair cost (damage state: extensive) & $70 \%$ of cost & Same as above \\
\hline Substation repair cost (damage state: complete) & $100 \%$ of cost & Same as above \\
\hline Annual maintenance cost (low voltage) & $\$ 30,000$ & Assumed \\
\hline Annual maintenance cost (medium voltage) & $\$ 60,000$ & Assumed \\
\hline Annual maintenance cost (high voltage) & $\$ 90,000$ & Assumed \\
\hline Discount rate & $4 \%$ & $\begin{array}{l}\text { Bastidas-Arteaga and Stewart } \\
\text { (2015) }\end{array}$ \\
\hline
\end{tabular}


The results of the life cycle cost analysis are presented in Table $8.6 \&$ Table 8.7. The net benefit, as well as the net benefit excluding societal economic losses, are plotted in Figure 8.10 \& Figure 8.11. It can be seen that the mitigation strategies reduce the repair cost, revenue loss, and the societal economic losses. Looking at the results for Charleston in Table 8.6 and Figure 8.10, it can be seen that only strategies 1 and 2, which entails hardening only substations, resulted in positive net benefit when societal economic losses are considered. Recall that strategy 1 entails strengthening 7 substations or $17 \%$ of the total substations while strategy 2 entails strengthening 16 substations or $39 \%$ of all substations. It can be seen from Table 8.6 and Figure 8.10 that strategy 2 yields more benefit than strategy 1 . Strategy 7 , which entails hardening the entire system, resulted in the highest reduction in losses. For example, the societal economic losses reduced from about $\$ 93$ million for the unhardened system to about $\$ 59$ million for strategy 7 . However, the high cost of implementing the mitigation strategy outweighs the total benefit which renders the strategy the most cost ineffective. 
Table 8.6 Cost analysis results for Charleston, SC (\$1000s)

\begin{tabular}{lcrrrrrrr}
\hline \hline Cost Category & $\begin{array}{c}\text { Unhardened } \\
\text { system }\end{array}$ & Strategy 1 & Strategy 2 & Strategy 3 & Strategy 4 & Strategy 5 & Strategy 6 & Strategy 7 \\
\hline Mitigation & - & 3,594 & 6,064 & 8,940 & 36,780 & 12,534 & 42,844 & 111,092 \\
Maintenance & 49,830 & 49,830 & 49,830 & 49,830 & 49,830 & 49,830 & 49,830 & 49,830 \\
Repair & 24,703 & 23,083 & 21,495 & 24,655 & 24,505 & 23,035 & 21,297 & 19,881 \\
Revenue loss & 1,127 & 1,062 & 1,007 & 1,120 & 1,112 & 1,055 & 993 & 714 \\
Societal economic loss & 93,282 & 87,900 & 83,376 & 92,718 & 92,051 & 87,336 & 82,145 & 59,050 \\
& & & & & & & & \\
LCC & $\mathbf{1 6 8 , 9 4 2}$ & $\mathbf{1 6 5 , 4 7 0}$ & $\mathbf{1 6 1 , 7 7 3}$ & $\mathbf{1 7 7 , 2 6 3}$ & $\mathbf{2 0 4 , 2 7 9}$ & $\mathbf{1 7 3 , 7 9 1}$ & $\mathbf{1 9 7 , 1 0 9}$ & $\mathbf{2 4 0 , 5 6 6}$ \\
LCC w/o societal loss & $\mathbf{7 5 , 6 6 1}$ & $\mathbf{7 7 , 5 6 9}$ & $\mathbf{7 8 , 3 9 7}$ & $\mathbf{8 4 , 5 4 6}$ & $\mathbf{1 1 2 , 2 2 8}$ & $\mathbf{8 6 , 4 5 4}$ & $\mathbf{1 1 4 , 9 6 4}$ & $\mathbf{1 8 1 , 5 1 6}$ \\
Net benefit (NB) & - & $\mathbf{3 , 4 7 3}$ & $\mathbf{7 , 1 6 9}$ & $\mathbf{- 8 , 3 2 1}$ & $\mathbf{- 3 5 , 3 3 6}$ & $\mathbf{- 4 , 8 4 8}$ & $\mathbf{- 2 8 , 1 6 7}$ & $\mathbf{- 7 1 , 6 2 4}$ \\
NB w/o societal loss & - & $\mathbf{- 1 , 9 0 9}$ & $\mathbf{- 2 , 7 3 7}$ & $\mathbf{- 8 , 8 8 5}$ & $\mathbf{- 3 6 , 5 6 7}$ & $\mathbf{- 1 0 , 7 9 4}$ & $\mathbf{- 3 9 , 3 0 4}$ & $\mathbf{- 1 0 5 , 8 5 6}$ \\
\hline
\end{tabular}

Table 8.7 Cost analysis results for New York, NY (\$1000s)

\begin{tabular}{|c|c|c|c|c|c|c|c|c|}
\hline Cost Category & $\begin{array}{l}\text { Unhardened } \\
\text { system }\end{array}$ & Strategy 1 & Strategy 2 & Strategy 3 & Strategy 4 & Strategy 5 & Strategy 6 & Strategy 7 \\
\hline Mitigation & - & 3,594 & 6,064 & 8,940 & 36,780 & 12,534 & 42,844 & 111,092 \\
\hline Maintenance & 49,830 & 49,830 & 49,830 & 49,830 & 49,830 & 49,830 & 49,830 & 49,830 \\
\hline Repair & 3,322 & 2,862 & 2,617 & 3,297 & 3,219 & 2,837 & 2,514 & 2,232 \\
\hline Revenue loss & 67 & 66 & 64 & 67 & 66 & 65 & 63 & 27 \\
\hline Societal economic loss & 5,582 & 5,496 & 5,315 & 5,505 & 5,444 & 5,418 & 5,177 & 2,236 \\
\hline $\mathbf{L C C}$ & 58,802 & 61,848 & 63,890 & 67,639 & 95,340 & 70,685 & 100,428 & 165,418 \\
\hline LCC w/o societal loss & 53,220 & 56,353 & 58,576 & 62,134 & $\mathbf{8 9 , 8 9 6}$ & 65,267 & 95,251 & 163,181 \\
\hline Net benefit (NB) & - & $-3,046$ & $-5,089$ & $-\mathbf{8 , 8 3 7}$ & $-36,538$ & $-11,883$ & $-41,627$ & $-106,616$ \\
\hline NB w/o societal loss & - & $-3,133$ & $-5,356$ & $-8,914$ & $-36,675$ & $-12,047$ & $-42,031$ & $-109,961$ \\
\hline
\end{tabular}




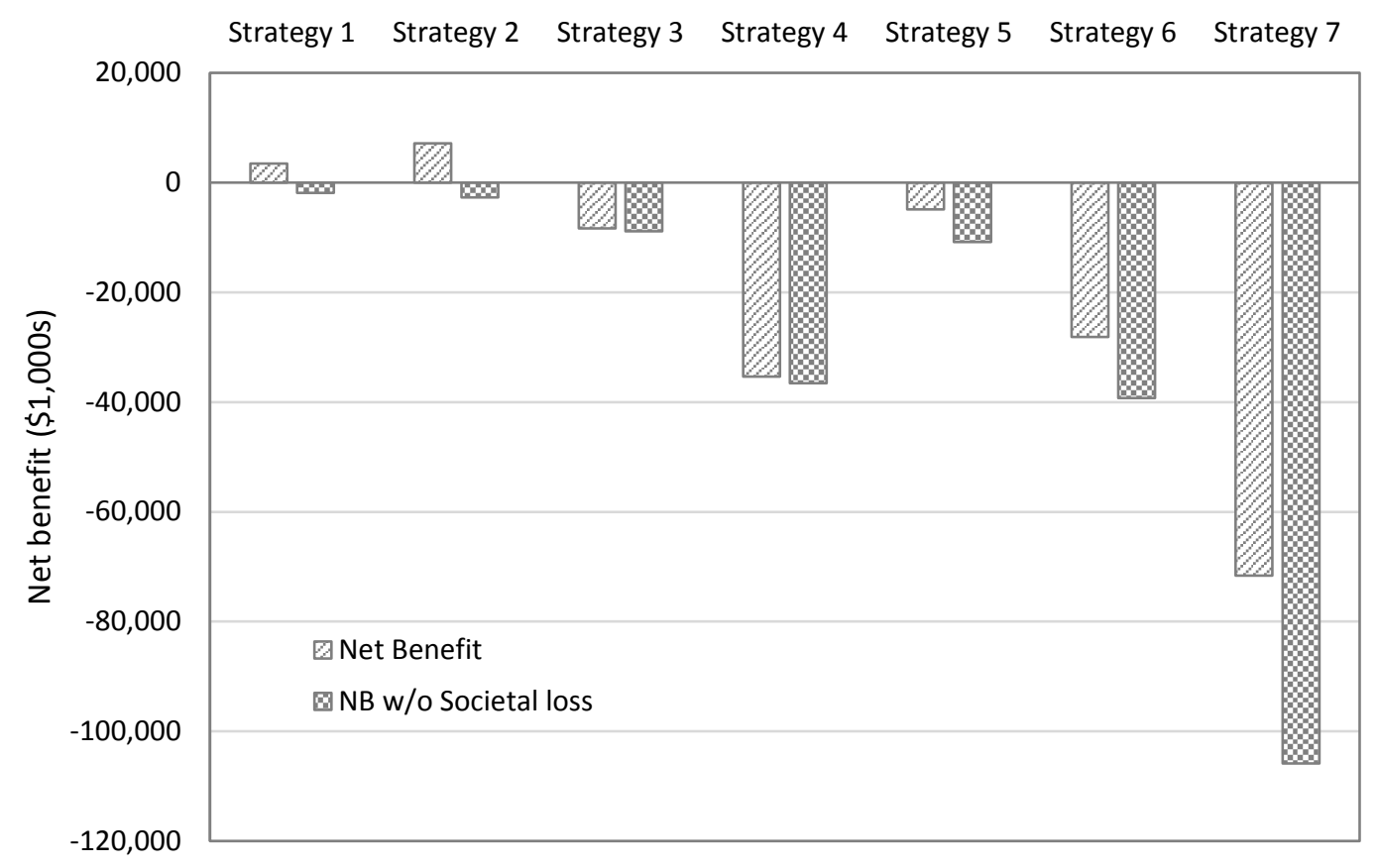

Figure 8.10 Net benefit of mitigation strategies (Charleston, SC)

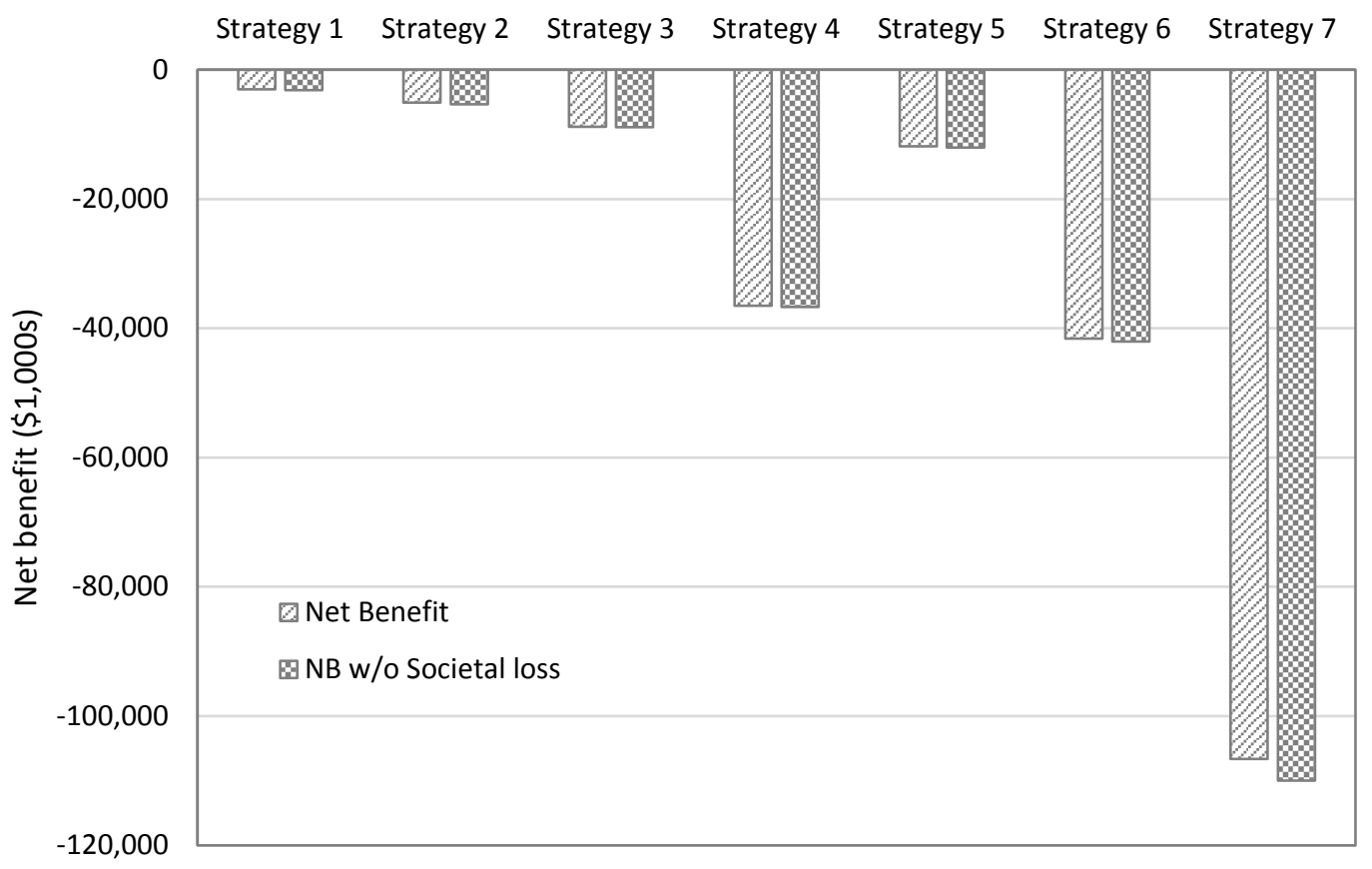

Figure 8.11 Net benefit of mitigation strategies (New York, NY) 
On the other hand, strategies 3 and 4, which entails hardening only transmission lines, are not cost effective in Charleston. This is because, based on the costs in Table 8.5, it is far more expensive to harden transmission lines than substations. With resources for mitigation strategies usually limited, it will be far more rewarding, economically, to invest in strengthening substations against seismic hazard in this case than strengthening transmission lines against hurricane hazard.

It can also be seen from Table 8.6 and Figure 8.10 that if the societal economic losses are not included in the analysis, none of the mitigation strategies is cost effective in Charleston, with the highest net benefit being $-\$ 1.9$ million for strategy 1 . The decision to include societal economic losses will depend on the entity carrying out the cost analysis. Privately owned utility companies might not consider societal economic losses in their analysis. Municipal utilities owned by city governments, on the other hand, will likely consider all aspects of the costs.

Comparing Charleston and New York, it can be seen that while some of the mitigation strategies are cost effective in Charleston, none is cost effective in New York. This is because both the seismic and hurricane hazard risks are lower in New York than Charleston. This is evident from the total life cycle cost (LCC) of the system in the two locations. For example, the LCC in Charleston for the unhardened system is about \$169 million, while the corresponding LCC in New York is about a third of that (about \$59 million). This implies that the system will be subjected to considerably higher risk from earthquakes and hurricanes in Charleston.

The mitigation strategies are largely ineffective, economically, due to the high cost of the mitigation strategies. As stated earlier, all the costs adopted in Table 8.5 are for demonstrating the proposed framework and might not be reflective of the actual costs in a particular location. 
If the discount rate of $4 \%$ in Table 8.5 is reduced by half to $2 \%$, i.e., the present value of future cost is increased, then mitigation strategies 1 and 2 are cost effective in Charleston with higher net benefit than using a discount rate of $4 \%$ as seen in Figure 8.12. For example, the net benefit of strategy 1 increased from about $\$ 3.5$ million to about $\$ 6.5$ million for discount rates of $4 \%$ and $2 \%$, respectively. Even with a discount rate of $2 \%$, none of the mitigation strategies is cost effective in New York as seen in Figure 8.13.

It is interesting to note that if the cost of mitigation for substations, which is assumed as $2 \%$ of substation cost in Table 8.5 , is changed to $5 \%$ of substation cost, none of the mitigation strategies is cost effective in both locations with a discount rate of $4 \%$.

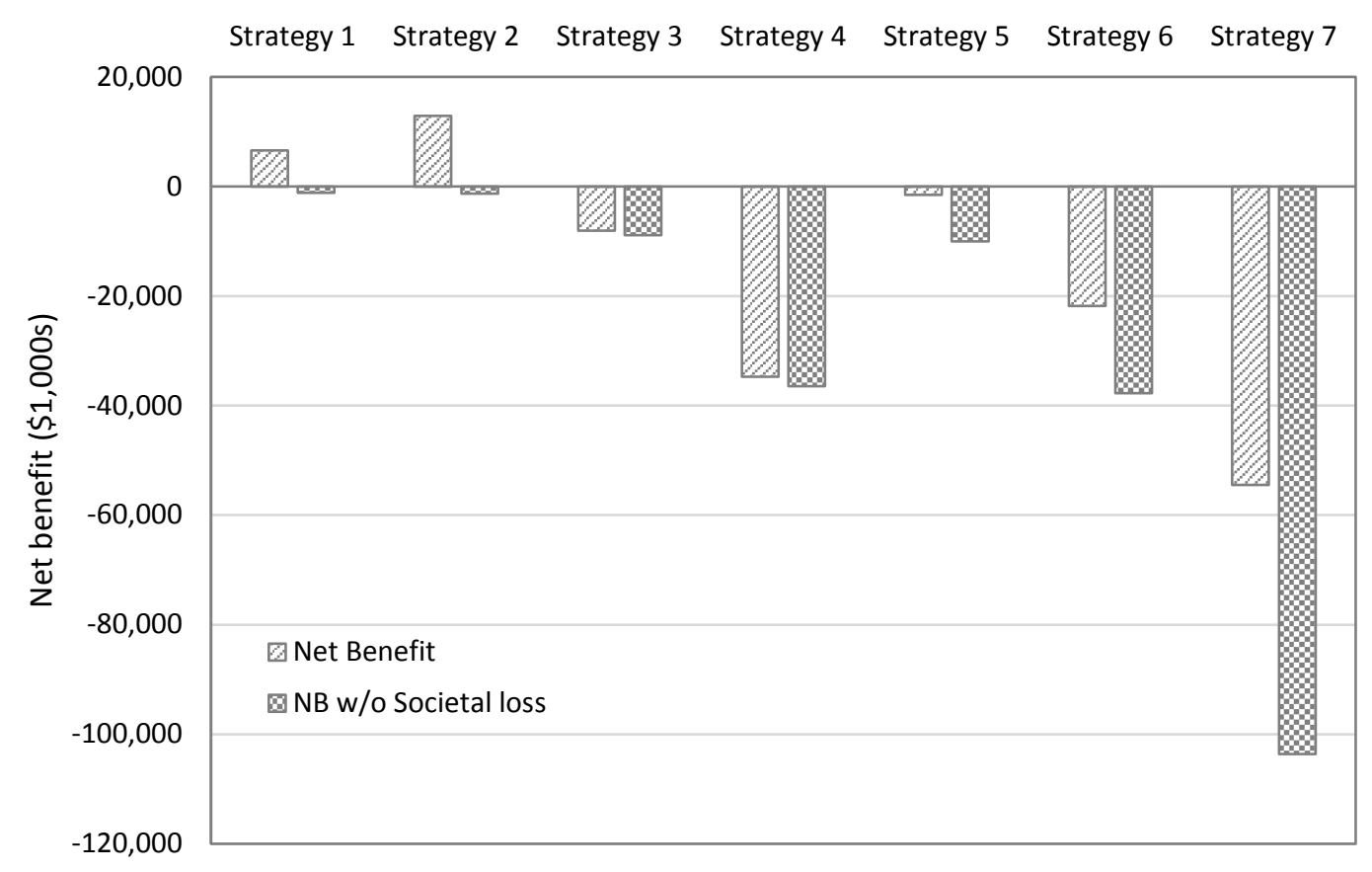

Figure 8.12 Net benefit of mitigation strategies with $2 \%$ discount rate (Charleston, SC) 


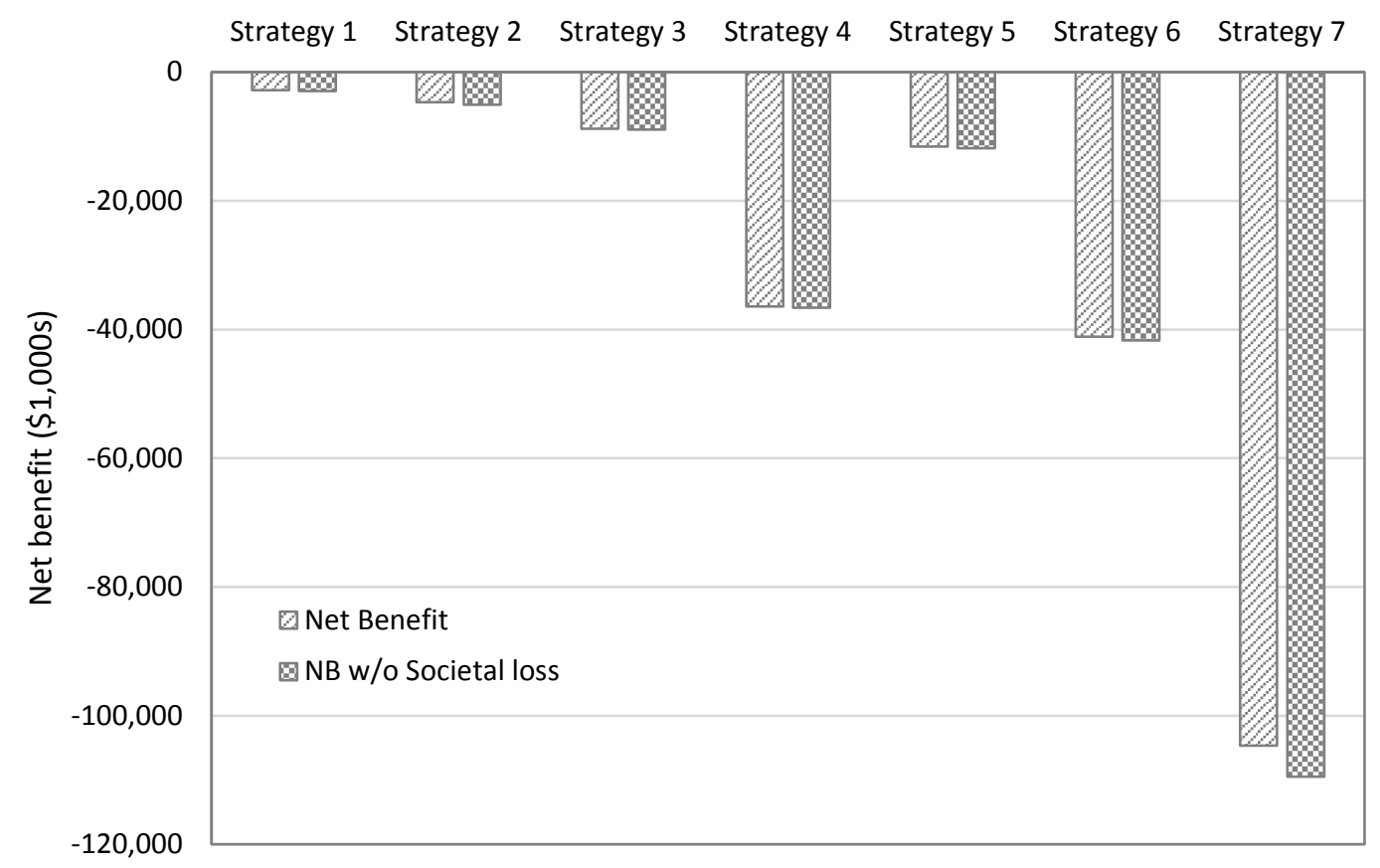

Figure 8.13 Net benefit of mitigation strategies with 2\% discount rate (New York, NY)

\subsection{Conclusions}

A framework for evaluating the effectiveness of multi-hazard risk mitigation strategies for electric power systems is presented. The framework consists of hazard analysis modeling through probabilistically weighted deterministic scenarios approach, component importance measure that considers multi-element failure, as well as a probabilistic lifecycle cost analysis model. The comprehensive framework can be used to take into account multiple hazards that can impact a system during its lifetime. The framework is demonstrated using a notional electric power network assumed to be located in Charleston, SC, and New York, NY.

The developed framework can be used by decision makers to prioritize investment on mitigation strategies in a case of competing hazards and limited resources. For example, it 
was shown based on the case study considered that it will be more cost effective to invest in seismic risk mitigation of substations in Charleston than it is to invest in hurricane risk mitigation of transmission lines.

It can be concluded that the cost effectiveness of multi-hazard risk mitigation strategies depends on the level of risk on the system in any given location. For regions with relatively moderate seismic and hurricane risks such as Charleston, certain mitigation strategies can be cost effective. For regions such as New York where both the seismic and hurricane hazard risks are relatively low, mitigation strategies might not be attractive, economically. However, mitigation strategies can lead to improvement in system reliability which can positively impact customer satisfaction and reduce power outage to critical facilities during disasters.

It can also be concluded based on the case study that whole scale hardening of a system is not cost effective. Rather, targeted hardening that entails identifying critical components of the system should be considered by utility companies. Critical components can be identified based on their impact on overall system reliability using the proposed component importance measure, or criticality can be evaluated based on facilities connected or served by a component. 


\section{Summary, Conclusions, Applications, and Future Work}

\subsection{Summary}

This research developed a framework for system-level risk assessment and management for electric power systems subjected to hurricanes and earthquakes. Parts of the framework include a topological-based system reliability model, probabilistic and scenario-based hazard analysis, climate change modeling, component vulnerability, component importance measure, multi-hazard risk assessment method, and cost analysis. Notional electric power systems assumed to be located in various regions of the U.S. were used to demonstrate the proposed framework. Several risk mitigation strategies were also proposed and their efficiency and cost-effectiveness studied. The research and its contributions are summarized below.

1. A topological-based system reliability method was developed for both radial topology typical for distribution systems as well as networked topology typical for transmission systems. This was done by formulating the accessibility of system components using reliability block diagrams, fault tree diagrams, and enumeration of minimal cut sets in a networked system.

2. A framework for targeted hardening of distribution systems subjected to hurricanes was presented. The framework can be used to identify critical parts of the systems for risk mitigation strategies. The framework was demonstrated using a distribution system assumed to be located in Florida.

3. The potential impact of climate change on hurricane hazard was modeled through a hurricane simulation model that allows the variation in both intensity and frequency to be considered. 
4. A multi-hazard risk assessment approach for electric power systems subjected to hurricanes and earthquakes was presented. A comparative approach using risk curves as well as a cumulative approach based on annualized risk were proposed.

5. A component importance measure appropriate for networked systems was proposed. The proposed measure considers multi-element failure to take into account redundancy in transmission networks as well as widespread damage observed during natural hazards.

6. A probabilistic-based cost analysis approach for multi-hazard risk assessment was presented to take into account the uncertainties in both hazard occurrence and damage estimation.

7. Several risk mitigation strategies were proposed and their efficiency in improving system reliability as well as their cost-effectiveness was evaluated.

\subsection{Conclusions}

Major conclusions and findings from the proposed framework and the case studies considered are summarized below.

1. The limitation of the topological-based system reliability method depends on the type of risk mitigation strategy being considered. For example, in the case of risk mitigation using stronger support structures for distribution and transmission systems, or anchoring substations, the system exist as it is and its engineering properties such as voltage are not changed. Consequently, the topological-based approach can appropriately model the damage in the system which is basically related to structural component strength. In other mitigation strategies, however, such as constructing new distribution lines to connect feeders from different 
substations, the applicability of the topological-based model is limited. Power flow analysis is required to determine whether a given substation can supply power to additional customers not in its primary area as well as check other factors such as voltage drop which can be prohibiting for the mitigation strategy.

2. Implementing risk mitigation strategies to entire systems is usually not costeffective. Identifying and strengthening critical components of a system can be cost-effective and should be considered by utility companies.

3. Designing and building stronger systems at the construction stage can be more costeffective than strengthening existing systems. As such, natural hazard risk mitigation strategies should be considered at the design stage of new systems.

4. Societal economic losses can constitute a huge percentage of total losses after a hazard event. Results from the risk assessment of all levels of power systems and all types of mitigation strategies considered point to the considerable impact of power outages to the economy. Policy makers and especially municipal utility companies owned by governments should consider such losses in any risk mitigation studies.

5. Considerable uncertainty exists in climate modeling and the impact of climate variation on hurricane hazard. As such, a scenario-based approach should be adopted by utility companies to study what might happen under different climate scenarios.

6. Multi-hazard risk assessment and management for electric power systems can be carried out using probabilistically weighted deterministic hazard scenarios approach. While this approach might not consider the entire range of possible hazard levels, it does allow the modeling of spatial variation of hazard intensity for distributed infrastructure systems. 
7. Finally, it can be noted from the formulations and results that considerable uncertainties exist in all stages of risk assessment of infrastructure systems subjected to natural hazards. From hazard occurrence, to damage quantification, to consequence analysis, aleatory and epistemic uncertainties exist. The aim, therefore, as summarized astutely by the quote below, is to find a basis for damage reduction.

"Only if we accept that complete prevention is ultimately unattainable, our rethinking of disasters leads us towards a policy of long-term loss reduction. And for this type of mitigation policy, the precise measure of risks (prediction of damages; assessment of uncertainty or complexity) might not be necessary, or even important. The crucial point is to provide the basis for damage reduction, i.e., to identify which areas are subject to different levels of potential damage and which factors determine such damage. This, in turn, can be used to identify the actions that must be taken to reduce future damage, even if we cannot quantify them exactly." - Weichselgartner (2001)

\subsection{Applications and Recommendations for Future Study}

In general, the proposed framework can be used for pre-disaster preparation, mitigation, and post-disaster response planning. The specific ways in which the framework can help achieve these are summarized below.

1. The proposed framework incorporates uncertainties inherent in risk due to natural hazards by adopting a probabilistic-based approach in hazard analysis, component vulnerable, and quantification of risk through a probabilistic system reliability measure. As such, the framework can be used as a tool for risk assessment to 
evaluate existing power systems or proposed new systems considering various sources of uncertainties.

2. The framework can also be used at the design stage to investigate the costeffectiveness of building a more reliable system than otherwise required.

3. The framework can be used to determine critical parts of the system to strengthen, compare the cost-effectiveness of various mitigation strategies, and prioritize risk from competing hazards.

4. Identification of critical systems components based on system topology can help decision making regarding post-disaster repairs which can lead to improvement in resilience.

5. The framework can also be used to make post-disaster decisions regarding whether to restore the system to its original state or to replace failed components with stronger, more reliable ones.

The present research has also helped to identify the following areas for future studies:

1. Electric power systems are critical to the operation of many infrastructure systems. Therefore, risk mitigation strategies employed for electric power systems will inevitably improve the performance of other infrastructure systems during natural hazard events. As such, cost savings from such improvement can be incorporated in future studies of the cost-effectiveness of mitigation strategies.

2. While the proposed framework can help with post-disaster response planning, a comprehensive method for systematic restoration of electric power systems is needed to improve resilience. Such method should consider not only component importance but restoration times and repair crew allocations. 
3. While the current study focused on hurricanes and earthquakes which are independent and non-concurrent hazards, there is a need to develop a framework for risk assessment of concurrent and/or dependent hazards such as earthquakes and tsunamis, and hurricanes and storm surge.

4. In this study, aging of wood distribution poles was considered. However, aging and deterioration of other system components need to be considered in a long-term risk assessment approach. This requires testing and data collection to be able to quantify deterioration in component strength and how it affects system functionality as well as costs.

5. Other natural hazards such as ice storms, floods, tornados etc. also pose a great threat to electric power systems across the world. Modeling of such hazards and the risk they pose needs to be explored.

6. Finally, a methodology for community resilience can be developed through an integrated risk assessment that considers the complex interdependent nature of modern infrastructure systems. 


\section{References}

Adachi, T., \& Ellingwood, B. R. (2010). Comparative assessment of civil infrastructure network performance under probabilistic and scenario earthquakes. Journal of Infrastructure Systems, 16(1), 1-10.

Albert, R., Albert, I., \& Nakarado, G. L. (2004). Structural vulnerability of the North American power grid. Physical review E, 69(2), 025103.

ANSI-O5.1. (2002). Timber Poles Specifications and Dimensions: American National Standards Institute.

ASCE-7. (2010). Minimum design loads for building and other structures: American Society of Civil Engineers, ASCE Reston Virginia.

ASCE-74. (1991). Guidelines for Electrical Transmission Line Structural Loading. Reston, VA: American Society of Civil Engineers.

ASCE-74. (2009). Guidelines for Electrical Transmission Line Structural Loading. Reston, VA: American Society of Civil Engineers.

ASCE-111. (2006). Reliability-Based Design of Utility Pole Structures: American Society of Civil Engineers.

ASCE. (2005). Minimum design loads for buildings and other structures. Reston, VA: American Society of Civil Engineers.

ASCE. (2013). 2013 report card for America's infrastructure. Reston, VA: American Society of Civil engineers

ASCE. (2015). Adapting Infrastructure and Civil Engineering Practice to a Changing Climate (R. Olsen Ed.). Reston, VA: American Society of Civil Engineers Committee on Adaptation to a Changing Climate.

ATC. (2015). Windspeed by Location. Retrieved 6/25, 2015, from http://windspeed.atcouncil.org/

ATS. (2014). Products and Pricing. Retrieved 11/30/2014, 2014, from http://www.americantimberandsteel.com/poles-pilings-utility-poles-unframedcca.html

Ayyub, B. M., Foster, J., \& McGill, W. L. (2009). Risk analysis of a protected hurricaneprone region. I: Model development. Natural Hazards Review, 10(2), 38-53. 
Bagchi, A. (2009). Modeling the power distribution network of a virtual city and studying the impact of fire on the electrical infrastructure. Texas A\&M University.

Bagchi, A., Sprintson, A., \& Singh, C. (2009, 4-6 Oct.). Modeling the impact of fire spread on the electrical distribution network of a virtual city. Paper presented at the North American Power Symposium (NAPS), 2009, Starkville, MS.

Balducci, P. J., Schienbein, L. A., Nguyen, T. B., Brown, D. R., \& Fatherlrahman, E. (2006). An examination of the costs and critical characteristics of electric utility distribution system capacity enhancement projects. Paper presented at the Transmission and Distribution Conference and Exhibition, 2005/2006 IEEE PES.

Bastidas-Arteaga, E., \& Stewart, M. G. (2015). Damage risks and economic assessment of climate adaptation strategies for design of new concrete structures subject to chloride-induced corrosion. Structural Safety, 52, Part A(0), 40-53. doi: http://dx.doi.org/10.1016/j.strusafe.2014.10.005

Bell, R., \& Glade, T. (2004). Multi-hazard analysis in natural risk assessments: WIT Press, Boston.

Bender, M. A., Knutson, T. R., Tuleya, R. E., Sirutis, J. J., Vecchi, G. A., Garner, S. T., \& Held, I. M. (2010). Modeled impact of anthropogenic warming on the frequency of intense Atlantic hurricanes. Science, 327(5964), 454-458.

Bernstein, L., Bosch, P., Canziani, O., Chen, Z., Christ, R., Davidson, O., . . Kattsov, V. (2007). IPCC, 2007: climate change 2007: synthesis report. Contribution of working groups I. II and III to the Fourth Assessment Report of the Intergovernmental Panel on Climate Change. Intergovernmental Panel on Climate Change, Geneva. $<$ http://www. ipcc. ch/ipccreports/ar4-syr. htm.

Bjarnadottir, S., Li, Y., \& Stewart, M. (2013). Hurricane Risk Assessment of Power Distribution Poles Considering Impacts of a Changing Climate. Journal of Infrastructure Systems, 19(1), 12-24.

Bjarnadottir, S., Li, Y., \& Stewart, M. G. (2014). Risk-based economic assessment of mitigation strategies for power distribution poles subjected to hurricanes. Structure and Infrastructure Engineering, 10(6), 740-752.

Blake, E. S., Kimberlain, T. B., Berg, R. J., Cangialosi, J. P., \& Beven, J. L. (2013). Tropical Cyclone Report - Hurricane Sandy. (AL182012).

Bradley, B. A., Dhakal, R. P., Cubrinovski, M., Mander, J. B., \& MacRae, G. A. (2007). Improved seismic hazard model with application to probabilistic seismic demand analysis. Earthquake engineering \& structural dynamics, 36(14), 2211-2225.

Brown, R. E. (2008). Electric power distribution reliability: CRC press. 
Brown, R. E. (2009). Cost-benefit analysis of the deployment of utility infrastructure upgrades and storm hardening programs. Raleigh: Quanta Technology.

Brumbelow, K., Torres, J., Guikema, S., Bristow, E., \& Kanta, L. (2007). Virtual cities for water distribution and infrastructure system research. Paper presented at the World Environmental and Water Resources Congress.

Cardona, O. D., Ordaz, M., Reinoso, E., Yamín, L., \& Barbat, A. (2012). CAPRAcomprehensive approach to probabilistic risk assessment: international initiative for risk management effectiveness. Paper presented at the Proceedings of the 15th world conference in earthquake engineering. Lisbon, Portugal.

Carpignano, A., Golia, E., Di Mauro, C., Bouchon, S., \& Nordvik, J. P. (2009). A methodological approach for the definition of multi-risk maps at regional level: first application. Journal of Risk Research, 12(3-4), 513-534.

Cavalieri, F., Franchin, P., Buriticá Cortés, J. A., \& Tesfamariam, S. (2014). Models for seismic vulnerability analysis of power networks: comparative assessment. Computer-Aided Civil and Infrastructure Engineering, 29(8), 590-607.

Chang, S. E. (2003). Evaluating disaster mitigations: methodology for urban infrastructure systems. Natural Hazards Review, 4(4), 186-196.

Chang, S. E., Shinozuka, M., \& Moore, J. E. (2000). Probabilistic earthquake scenarios: extending risk analysis methodologies to spatially distributed systems. Earthquake Spectra, 16(3), 557-572.

Chen, S. C., Gulati, S., Hamid, S., Huang, X., Luo, L., Morisseau-Leroy, N., . . Zhang, C. (2004). A Web-based distributed system for hurricane occurrence projection. Software: Practice and Experience, 34(6), 549-571.

Chouhan, S., Wan, H., Lai, H., Feliachi, A., \& Choudhry, M. (2009). Intelligent reconfiguration of smart distribution network using multi-agent technology. Paper presented at the Power \& Energy Society General Meeting, 2009. PES'09. IEEE.

Coles, S., \& Simiu, E. (2003). Estimating uncertainty in the extreme value analysis of data generated by a hurricane simulation model. Journal of engineering mechanics, 129(11), 1288-1294.

Cornell, C. A., Jalayer, F., Hamburger, R. O., \& Foutch, D. A. (2002). Probabilistic basis for 2000 SAC federal emergency management agency steel moment frame guidelines. Journal of Structural Engineering, 128(4), 526-533.

Creager, K., Crosson, R., Pratt, T., Weaver, C., Kramer, S., MacRae, G., . . . Chang, S. (2001). The Nisqually Earthquake of 28 February 2001: Preliminary Reconnaissance Report: University of Washington. 
CVSCC. (2004). Placement of utility distribution lines underground, Report to the Governor and the General Assembly of Virginia. Richmond, VA: Commonwealth of Virginia State Corporation Commission.

Datla, S., \& Pandey, M. (2006). Estimation of life expectancy of wood poles in electrical distribution networks. Structural Safety, 28(3), 304-319.

Davidson, R. A., Liu, H., Sarpong, K., Sparks, P., \& Rosowsky, D. V. (2003). Electric power distribution system performance in Carolina Hurricanes. Natural Hazards Review, 4(1), 36-45. doi: 10.1061/(ASCE)1527-6988(2003)4:1(36)

Davis, M., \& Clemmer, S. (2014). Power Failure: How climate change puts our electricity at risk - and what we can do about it: Union of Concerned Scientists.

DeMaria, M., Knaff, J. A., \& Kaplan, J. (2006). On the decay of tropical cyclone winds crossing narrow landmasses. Journal of applied meteorology and climatology, 45(3), 491-499.

DOE. (2016). Electric Disturbance Events (OE-417) Annual Summaries. from U.S. Department of Energy https://www.oe.netl.doe.gov/OE417_annual_summary.aspx

Dong, X., Shinozuka, M., \& Chang, S. (2004). Utility power network systems. Paper presented at the Proc. of 13th World Conference on Earthquake Engineering.

Duenas-Osorio, L., \& Hernandez-Fajardo, I. (2008). Flow-based reliability assessment of infrastructure systems. Paper presented at the 14th World Conference on Earthquake Engineering (14WCEE).

Dueñas-Osorio, L., Craig, J. I., \& Goodno, B. J. (2007). Seismic response of critical interdependent networks. Earthquake engineering \& structural dynamics, 36(2), $285-306$.

EIA. (2013). 2012 Utility Bundled Retail Sales. Retrieved 11/28/2014, from U.S. Energy Information Administration http://www.eia.gov/electricity/data.cfm

EIA. (2016a). Annual Energy Review. from U.S. Energy Information Administration http://www.eia.gov/totalenergy/data/annual/\#consumption

EIA. (2016b). Electric Sales, Revenue, and Average Price. from U.S. Energy Information Administration http://www.eia.gov/electricity/sales_revenue price/

Eidinger, J. (2009). Wenchuan earthquake impact to power systems. TCLEE 2009, Lifeline Earthquake Engineering in a Multihazard Environment, 1359-1370.

Eidinger, J., \& Kempner, J., L. (2012). Risk Assessment of Transmission System under Earthquake Loading. Paper presented at the Electrical Transmission and Substation Structures 2012@sSolutions to Building the Grid of Tomorrow. 
Ellingwood, B. R., \& Tekie, P. B. (1999). Wind load statistics for probability-based structural design. Journal of Structural Engineering, 125(4), 453-463.

Ellingwood, B. R., \& Wen, Y. K. (2005). Risk-benefit-based design decisions for lowprobability/high consequence earthquake events in Mid-America. Progress in Structural Engineering and Materials, 7(2), 56-70.

Elsner, J. B., Kossin, J. P., \& Jagger, T. H. (2008). The increasing intensity of the strongest tropical cyclones. Nature, 455(7209), 92-95.

Enacheanu, B., Raison, B., Caire, R., Devaux, O., Bienia, W., \& Hadjsaid, N. (2008). Radial network reconfiguration using genetic algorithm based on the matroid theory. Power Systems, IEEE Transactions on, 23(1), 186-195.

EPRI, DOE, \& NRC. (2012). Technical Report: Central and Eastern United States Seismic Source Characterization for Nuclear Facilities. Palo Alto, CA: Electric Power Research Institute (EPRI), U.S. Department of Energy (U.S. DOE), U.S. Nuclear Regulatory Commission (U.S. NRC).

Esary, J., \& Proschan, F. (1963). Coherent structures of non-identical components. Technometrics, 5(2), 191-209.

ESDU. (1982). Strong winds in the atmospheric boundary layer, Part 1: Mean hourly wind speed. London: Engineering Sciences Data Unit.

ESDU. (1983). Strong winds in the atmospheric boundary layer, Part 1: Discrete gust speeds. London: Engineering Sciences Data Unit.

Ezell, B. C., Farr, J. V., \& Wiese, I. (2000). Infrastructure risk analysis model. Journal of Infrastructure Systems, 6(3), 114-117.

Faza, A. Z., Sedigh, S., \& McMillin, B. M. (2007). Reliability modeling for the advanced electric power grid: Springer.

FEMA. (2005). Multi-Hazard Loss Estimation Methodology, Flood Model: Hazus-MH MR2 Technical Manual: FEMA Washington, DC.

FEMA. (2009). NEHRP Recommended Seismic Provisions for New Buildings and Other Structures: FEMA P-750/2009 Edition. Washington, DC: Federal Emergency Management Agency.

FEMA. (2010). Multi-Hazard Loss Estimation Methodology, Earthquake Model: HazusMH 2.1 Technical Manual. Washington, DC: FEMA.

FEMA. (2011). Multi-Hazard Loss Estimation Methodology, Hurricane Model: HazusMH 2.1 Technical Manual. Washington, DC: FEMA. 
FPSC. (2005). Preliminary analysis of placing investor-owned electric utility transmission and distribution facilities underground in Florida. Tallahassee, FL: Florida Public Service Commission.

Francis, R. A., Falconi, S. M., Nateghi, R., \& Guikema, S. D. (2011). Probabilistic life cycle analysis model for evaluating electric power infrastructure risk mitigation investments. Climatic change, 106(1), 31-55.

Fussell, J. (1975). How to hand-calculate system reliability and safety characteristics. IEEE Transactions on Reliability, 3, 169-174.

Georgiou, P. N., Davenport, A. G., \& Vickery, B. (1983). Design wind speeds in regions dominated by tropical cyclones. Journal of Wind Engineering and Industrial Aerodynamics, 13(1), 139-152.

Gere, J. M., \& Carter, W. O. (1962). Critical buckling loads for tapered columns. ASCE -Proceedings -- Journal of the Structural Division, 88(ST1, Part 1), 1-11.

Gonen, T. (2014). Electric power distribution engineering: CRC press.

Gregory, L. B., Willoughby, R. L., Hodgin, D. S., Buckner, H. G., Watson, W., Taylor, H. M., \& Rant, P. J. (2006). Cost-effectiveness of undergrounding electric distribution facilities in Florida: Municipal Underground Utilities Consortium.

Gustavsen, B., \& Rolfseng, L. (2000). Simulation of wood pole replacement rate and its application to life cycle economy studies. IEEE Transactions on Power Delivery, 15(1), 300-306. doi: $10.1109 / 61.847266$

Haimes, Y. Y. (2015). Risk modeling, assessment, and management: John Wiley \& Sons.

Han, S.-R., Rosowsky, D., \& Guikema, S. (2013). Integrating Models and Data to Estimate the Structural Reliability of Utility Poles During Hurricanes. doi: 10.1111/risa.12102

Hines, P., Apt, J., \& Talukdar, S. (2009). Large blackouts in North America: Historical trends and policy implications. Energy Policy, 37(12), 5249-5259.

Hoffman, P., Bryan, W., \& Lippert, A. (2009). Comparing the Impacts of the 2005 and 2008 Hurricanes on US Energy Infrastructure. US Department of Energy.

Holland, G. J. (1980). An analytic model of the wind and pressure profiles in hurricanes. Monthly weather review, 108(8), 1212-1218.

Huang, Z., Rosowsky, D., \& Sparks, P. (2001a). Hurricane simulation techniques for the evaluation of wind-speeds and expected insurance losses. Journal of Wind Engineering and Industrial Aerodynamics, 89(7), 605-617. 
Huang, Z., Rosowsky, D. V., \& Sparks, P. R. (2001b). Long-term hurricane risk assessment and expected damage to residential structures. Reliability Engineering \& System Safety, 74(3), 239-249. doi: http://dx.doi.org/10.1016/S0951-8320(01)00086-2

IEEE. (2012). Natinal Electrical Safety Code: IEEE.

IPCC. (2000). Special report on emissions scenarios. Special Report on Emissions Scenarios, Edited by Nebojsa Nakicenovic and Robert Swart, pp. 612. ISBN 0521804930. Cambridge, UK: Cambridge University Press, July 2000.

IPCC. (2013). Summary for Policymakers. In: Climate Change 2013: The Physical Science Basis. Contribution of Working Group I to the Fifth Assessment Report of the Intergovernmental Panel on Climate Change [Stocker, T.F., D. Qin, G.-K. Plattner, M. Tignor, S.K. Allen, J. Boschung, A. Nauels, Y. Xia, V. Bex and P.M. Midgley (eds.)]. Cambridge University Press, Cambridge, United Kingdom and New York, NY, USA.

Jagger, T., Elsner, J. B., \& Niu, X. (2001). A dynamic probability model of hurricane winds in coastal counties of the United States. Journal of applied meteorology, 40(5), 853863.

Jagger, T. H., \& Elsner, J. B. (2006). Climatology models for extreme hurricane winds near the United States. Journal of Climate, 19(13), 3220-3236.

Jalayer, F. (2003). Direct probabilistic seismic anaysis: implementing non-linear dynamic assessments. Stanford University.

Jarrell, J. D., Mayfield, M., Rappaport, E. N., Landsea, C. W., Jarrell, J., Mayfield, M., . . . Landsea, C. (2001). The deadliest, costliest, and most intense United States hurricanes from 1900 to 2000 (and other frequently requested hurricane facts). NOAA, Technical Memorandum NWS-TPC-3.

Jayaram, N., \& Baker, J. (2010). Probabilistic seismic lifeline risk assessment using efficient sampling and data reduction techniques. Stanford University.

Johansson, J., Jonsson, H., \& Johansson, H. (2007). Analysing the vulnerability of electric distribution systems: a step towards incorporating the societal consequences of disruptions. International Journal of Emergency Management, 4(1), 4-17.

Kameshwar, S., \& Padgett, J. E. (2014). Multi-hazard risk assessment of highway bridges subjected to earthquake and hurricane hazards. Engineering Structures, 78, 154166.

Kaplan, J., \& DeMaria, M. (1995). A simple empirical model for predicting the decay of tropical cyclone winds after landfall. Journal of applied meteorology, 34(11), 24992512. 
Kaplan, J., \& DeMaria, M. (2001). On the decay of tropical cyclone winds after landfall in the New England area. Journal of applied meteorology, 40(2), 280-286.

Kaplan, S. (1997). The words of risk analysis. Risk Analysis, 17(4), 407-417.

Kappes, M. S., Keiler, M., von Elverfeldt, K., \& Glade, T. (2012). Challenges of analyzing multi-hazard risk: a review. Natural Hazards, 64(2), 1925-1958.

Kassakian, J. G., Schmalensee, R., Desgroseilliers, G., Heidel, T. D., Afridi, K., Farid, A., . . Kirtley, J. (2011). The future of the electric grid. Massachusetts Institute of Technology, Tech. Rep.

Kersting, W. H. (2012). Distribution system modeling and analysis: CRC press.

Kim, Y., \& Kang, W.-H. (2013). Network reliability analysis of complex systems using a non-simulation-based method. Reliability Engineering \& System Safety, 110, 8088.

Knabb, R. D., Rhome, J. R., \& Brown, D. P. (2005). Tropical Cyclone Report - Hurricane Katrina: National Hurricane Center.

Knight, B., \& Kempner Jr, L. (2009a). Seismic vulnerabilities and retrofit of high-voltage electrical substation facilities. Paper presented at the Proceedings of the 2009 Technical Council on Lifeline Earthquake Engineering conference. TCLEE. Oakland, California.

Knight, B. T., \& Kempner Jr, L. (2009b). Seismic Vulnerabilities and Retrofit of HighVoltage Electrical Substation Facilities. LifelineEarthquake Engineering in a Multihazard Environment, California, United States, 232-243.

Knutson, T. R., McBride, J. L., Chan, J., Emanuel, K., Holland, G., Landsea, C., . . Sugi, M. (2010). Tropical cyclones and climate change. Nature Geoscience, 3(3), 157163.

LaCommare, K. H., \& Eto, J. H. (2006). Cost of power interruptions to electricity consumers in the United States (US). Energy, 31(12), 1845-1855.

Landsea, C. W., Vecchi, G. A., Bengtsson, L., \& Knutson, T. R. (2010). Impact of Duration Thresholds on Atlantic Tropical Cyclone Counts*. Journal of Climate, 23(10), 2508-2519.

LaRocca, S., Johansson, J., Hassel, H., \& Guikema, S. (2014). Topological performance measures as surrogates for physical flow models for risk and vulnerability analysis for electric power systems. Risk Analysis, 35(4), 608-623. doi: 10.1111/risa.12281 
Lawrence, M. B., \& Cobb, H. D. (2005). Tropical Cyclone Report Hurricane Jeanne. National Oceanic and Atmospheric Association, http://www. nhc. noaa. gov/2004jeanne. shtml.

Li, Y. (2012). Assessment of Damage Risks to Residential Buildings and Cost-Benefit of Mitigation Strategies Considering Hurricane and Earthquake Hazards. Journal of Performance of Constructed Facilities, 26(1), 7-16.

Li, Y., Ahuja, A., \& Padgett, J. E. (2012). Review of methods to assess, design for, and mitigate multiple hazards. Journal of Performance of Constructed Facilities, 26(1), 104-117.

Li, Y., \& Ellingwood, B. R. (2006). Hurricane damage to residential construction in the US: Importance of uncertainty modeling in risk assessment. Engineering Structures, 28(7), 1009-1018.

Li, Y., \& Ellingwood, B. R. (2009). Framework for multihazard risk assessment and mitigation for wood-frame residential construction. Journal of Structural Engineering, 135(2), 159-168.

Li, Y., Yeddanapudi, S., McCalley, J. D., Chowdhury, A. A., \& Moorehead, M. (2005, 2325 Oct. 2005). Degradation-path model for wood pole asset management. Paper presented at the Proceedings of the 37th Annual North American Power Symposium, 2005. .

Lin, N., Emanuel, K., Smith, J., \& Vanmarcke, E. (2010). Risk assessment of hurricane storm surge for New York City. Journal of Geophysical Research: Atmospheres, 115(D18).

LIPA. (2005). A review of electric utility undergrounding policies and practices. Long Island, NY: Long Island Power Authority.

Lowrance, W. W. (1976). Of Acceptable Risk: Science and the Determination of Safety.

Mankowski, M., Hansen, E., \& Morrell, J. (2002). Wood pole purchasing, inspection, and maintenance: A survey of utility practices. Forest Products Journal, 52(11/12), 4350 .

Mann, M. E., \& Emanuel, K. A. (2006). Atlantic hurricane trends linked to climate change. Eos, 87(24), 233-244.

Mann, M. E., Sabbatelli, T. A., \& Neu, U. (2007). Evidence for a modest undercount bias in early historical Atlantic tropical cyclone counts. Geophysical Research Letters, $34(22)$. 
Marzocchi, W., Garcia-Aristizabal, A., Gasparini, P., Mastellone, M. L., \& Di Ruocco, A. (2012). Basic principles of multi-risk assessment: a case study in Italy. Natural Hazards, 62(2), 551-573.

Massie, A., \& Watson, N. R. (2011). Impact of the Christchurch earthquakes on the electrical power system infrastructure. Bulletin of the New Zealand Society for Earthquake Engineering, 44(4), 425-430.

Mendiluce, M. (2014). Risky Business: Building a Resilient Power Sector. Power and Energy Magazine, IEEE, 12(5), 34-41. doi: 10.1109/MPE.2014.2331892

Ming, X., Xu, W., Li, Y., Du, J., Liu, B., \& Shi, P. (2015). Quantitative multi-hazard risk assessment with vulnerability surface and hazard joint return period. Stochastic Environmental Research and Risk Assessment, 29(1), 35-44.

Morrell, J. J. (2008). Estimated service life of wood poles. Technical Bulletin, North American Wood Pole Council, http://www. woodpoles. org/documents/TechBulletin_EstimatedServiceLifeofWoodPole_12-08. pdf (Last accessed 5 April 2013).

Mosquera-Machado, S., \& Dilley, M. (2009). A comparison of selected global disaster risk assessment results. Natural Hazards, 48(3), 439-456.

Mudd, L., Wang, Y., Letchford, C., \& Rosowsky, D. (2014a). Assessing climate change impact on the US East Coast hurricane hazard: temperature, frequency, and track. Natural Hazards Review, 15(3).

Mudd, L., Wang, Y., Letchford, C., \& Rosowsky, D. (2014b). Hurricane wind hazard assessment for a rapidly warming climate scenario. Journal of Wind Engineering and Industrial Aerodynamics, 133(0), 242-249. doi: http://dx.doi.org/10.1016/j.jweia.2014.07.005

NERC. (2015). Electricity supply and demand: North American Electric Reliability Corporation.

NESC. (2002). National Electric Safety Code: Institute of Electrical and Electronics Engineers.

NOAA. (1990). Natural Disaster Survey Report: Hurricane Hugo. Silver Springs, MD: National Oceanic and Atmospheric Agency.

NOAA. (2005). Hurricane Rita: National Oceanic and Atmospheric Administration.

NOAA. (2015a). Chronological List of All Hurricanes: 1851 - 2014. Retrieved from: http://www.aoml.noaa.gov/hrd/hurdat/All U.S. Hurricanes.html?print=yes 
NOAA. (2015b). Continental U.S. Hurricanes. Retrieved 05/10/2015, 2015, from http://www.aoml.noaa.gov/hrd/hurdat/UShurrs detailed.html

NOAA. (2015c). The Saffir/Simpson Hurricane Scale. Retrieved 6/18/2015, 2015, from http://www.ncdc.noaa.gov/extremeevents/specialreports/saffir-simpson-hurricanescale.pdf

NOAA. (2016a). Defining a Hurricane. Retrieved 6/19, 2016, from http://www.aoml.noaa.gov/general/lib/defining.html

NOAA. (2016b). Hurricane direct hits on the mainland U.S. coastline and for individual states by Saffir/Simpson category. Retrieved 6/19, 2016, from http://www.aoml.noaa.gov/hrd/tcfaq/E19.html

NOAA. (2016c). Saffir-Simpson Hurricane Wind Scale. Retrieved 6/19, 2016, from http://www.nhc.noaa.gov/aboutsshws.php

Noda, M. (2001a). Disaster and restoration of electricity supply system by Hanshin-Awaji earthquake. Paper presented at the APEC Seminar on Earthquake Disaster Management of Energy Supply Systems, Taipei, China.

Noda, M. (2001b). Disaster and restoration of electricity supply system by Hanshin-Awaji Earthquake. Paper presented at the Proceedings, Seminar on Earthquake Disaster Management of Energy Supply System. Chinese Taipei: Earthquake Response Cooperation Program for Energy Supply Systems.

NRC. (1994). PRACTICAL LESSONS FROM THE LOMA PRIETA EARTHQUAKE. Washington, DC: National Research Council.

Ouyang, M., \& Dueñas-Osorio, L. (2011). An approach to design interface topologies across interdependent urban infrastructure systems. Reliability Engineering \& System Safety, 96(11), 1462-1473.

Ouyang, M., \& Dueñas-Osorio, L. (2014). Multi-dimensional hurricane resilience assessment of electric power systems. Structural Safety, 48(0), 15-24.

Ouyang, M., Dueñas-Osorio, L., \& Min, X. (2012). A three-stage resilience analysis framework for urban infrastructure systems. Structural Safety, 36, 23-31.

Pasch, R. J., Blake, E. S., III, H. D. C., \& Roberts, D. P. (2006). Tropical Cyclone Report - Hurricane Wilma: National Hurricane Center.

Pasch, R. J., Brown, D. P., \& Blake, E. S. (2005). Tropical cyclone report: Hurricane charley National Hurricane Center. 
Petersen, M. D., Moschetti, M. P., Powers, P. M., Mueller, C. S., Haller, K. M., Frankel, A. D., . . . Boyd, O. S. (2014). Documentation for the 2014 update of the United States national seismic hazard maps: US Geological Survey.

Philipson, L., \& Willis, H. L. (2006). Understanding electric utilities and de-regulation (2nd ed.). Boca Raton, FL: CRC Press.

Powell, M., Soukup, G., Cocke, S., Gulati, S., Morisseau-Leroy, N., Hamid, S., . . Axe, L. (2005). State of Florida hurricane loss projection model: Atmospheric science component. Journal of Wind Engineering and Industrial Aerodynamics, 93(8), 651674.

Ramos, E. R., Expósito, A. G., Santos, J. R., \& Iborra, F. L. (2005). Path-based distribution network modeling: application to reconfiguration for loss reduction. Power Systems, IEEE Transactions on, 20(2), 556-564.

Rausand, M., \& Høyland, A. (2004). System reliability theory: models, statistical methods, and applications (Vol. 396): John Wiley \& Sons.

Robert, W. D. (2002). Geotechnical Earthquake Engineering Handbook. NY: McGrawHill.

Romero, N., Nozick, L. K., Dobson, I., Xu, N., \& Jones, D. A. (2015). Seismic retrofit for electric power systems. Earthquake Spectra, 31(2), 1157-1176.

Ryan, P. C., Stewart, M. G., Spencer, N., \& Li, Y. (2014a). Reliability assessment of power pole infrastructure incorporating deterioration and network maintenance. Reliability Engineering \& System Safety, 132, 261-273.

Ryan, P. C., Stewart, M. G., Spencer, N., \& Li, Y. (2014b). Reliability assessment of power pole infrastructure incorporating deterioration and network maintenance. Reliability Engineering \& System Safety, 132(0), 261-273. doi: http://dx.doi.org/10.1016/j.ress.2014.07.019

Salman, A. M., \& Li, Y. (2015). Age-dependent fragility and life-cycle cost analysis of wood and steel power distribution poles subjected to hurricanes. Structure and Infrastructure Engineering, 1-14. doi: 10.1080/15732479.2015.1053949

Salman, A. M., \& Li, Y. (2016). Assessing Climate Change Impact on System Reliability of Power Distribution Systems Subjected to Hurricanes. Journal of Infrastructure Systems. doi: 10.1061/(ASCE)IS.1943-555X.0000316

Salman, A. M., Li, Y., \& Stewart, M. G. (2015). Evaluating system reliability and targeted hardening strategies of power distribution systems subjected to hurricanes. Reliability Engineering \& System Safety, 144, 319-333. 
Schiff, A., Tognazzini, R., \& Ostrom, D. (1995). Power Systems" in Northridge Earthquake-Lifeline Performance and Post-Earthquake Response: Technical Council on Lifeline Earthquake Engineering, American Society of Civil Engineers.

Sewell, R. T., Toro, G. R., \& McGuire, R. K. (1996). Impact of ground motion characterization on conservatism and variability in seismic risk estimates: Division of Engineering Technology, Office of Nuclear Regulatory Research, US Nuclear Regulatory Commission.

Shafieezadeh, A., Onyewuchi, P. U., Begovic, M. M., \& DesRoches, R. (2014a). Fragility Assessment of Wood Poles in Power Distribution Networks against Extreme Wind Hazards. Bridges, 10, 9780784412626.9780784412074.

Shafieezadeh, A., Onyewuchi, U. P., Begovic, M. M., \& DesRoches, R. (2014b). AgeDependent Fragility Models of Utility Wood Poles in Power Distribution Networks Against Extreme Wind Hazards. Power Delivery, IEEE Transactions on, 29(1), 131-139.

Shelby-County. (2015). Electricity, Gas \& Water. Retrieved 10/13, 2015, from https://www.shelbycountytn.gov/index.aspx?NID=503

Shinozuka, M., Dong, X., Jin, X., \& Cheng, T. (2005). Seismic performance analysis for the ladwp power system. Paper presented at the Transmission and Distribution Conference and Exhibition: Asia and Pacific, 2005 IEEE/PES.

Shinozuka, M., Rose, A., \& Eguchi, R. (1998). Engineering and socioeconomic impacts of earthquakes. Buffalo: MCEER.

Short, T. A. (2006). Electric Power Distribution Equipment and Systems. Boca Raton, FL: CRC Press.

Song, J., \& Ok, S. Y. (2010). Multi-scale system reliability analysis of lifeline networks under earthquake hazards. Earthquake engineering \& structural dynamics, 39(3), 259-279.

Staid, A., Guikema, S., Nateghi, R., Quiring, S., \& Gao, M. (2014). Simulation of tropical cyclone impacts to the U.S. power system under climate change scenarios. Climatic change, 1-12. doi: 10.1007/s10584-014-1272-3

Stewart, A. J. (1996, March 25-27). Utility poles: how long do they last. Paper presented at the Proceeding of the Conference on Poles and Piles, Fort Collins, CO.

Stewart, M., Wang, X., \& Willgoose, G. (2014). Direct and Indirect Cost-and-Benefit Assessment of Climate Adaptation Strategies for Housing for Extreme Wind Events in Queensland. Natural Hazards Review, 15(4), 04014008. 
Stewart, S. R. (2004). Tropical Cyclone Report: Hurricane Ivan: National Hurricane Center.

Taras, A., Ratel, G., \& Chouinard, L. (2004). A life-cycle cost approach to the maintenance of overhead line supports. Paper presented at the Reliability and Optimization of Structural Systems: Proceedings of the 11th IFIP WG7. 5 Working Conference, Banff, Canada, 2-5 November 2003.

TDA. (2006). Undergrounding feasibility study, final report. Truckee, CA: Tahoe Donner Association.

Tierney, K. J., Chang, S. E., Eguchi, R. T., \& Shinozuka, M. (1999). Improving earthquake loss estimation: Review, assessment and extension of loss estimation methodologies. Research Progress and, 12.

Toro, G. R., Abrahamson, N. A., \& Schneider, J. F. (1997). Model of strong ground motions from earthquakes in central and eastern North America: best estimates and uncertainties. Seismological research letters, 68(1), 41-57.

Toth, Z., \& Szentimrey, T. (1990). The binormal distribution: A distribution for representing asymmetrical but normal-like weather elements. Journal of Climate, $3(1), 128-136$.

U.S. Congress, O. o. T. A. (1990). Office of Technology Assessment. Physical vulnerability of electric system to natural disasters and sabotage: OTA-E-453, Washington, DC: US Government Printing Office.

UN. (2002). Johannesburg plan of implementation of the world summit on sustainable development: United Nations.

USDOE. (2005). Florida State's Energy Emergency Response to the 2004 Hurricanes: US Department of Energy - Office of Electricity Delivery and Energy Reliability.

USGS. (2008). 2008 Interactive Deaggregations. Retrieved from http://geohazards.usgs.gov/deaggint/2008/

USGS. (2015a). Hazard Curve Application. Retrieved 09/25, 2015, from http://geohazards.usgs.gov/hazardtool/application.php

USGS. (2015b). Historic Earthquakes. Retrieved 10/13, 2015, from http://earthquake.usgs.gov/earthquakes/states/events/1886 09_01.php

USGS. (2016). National Seismic Hazard Maps. Retrieved 6/19, 2016, from http://earthquake.usgs.gov/hazards/products/conterminous/index.php\#2008 
Valencia, N., Simone, S., \& Morrison, G. (2010). 6.5 earthquake strikes off California coast, $\quad C N N$. Retrieved from http://edition.cnn.com/2010/US/01/09/california.earthquake/index.html

Vanzi, I. (1996). Seismic reliability of electric power networks: methodology and application. Structural Safety, 18(4), 311-327.

Vickery, P. J., Masters, F. J., Powell, M. D., \& Wadhera, D. (2009a). Hurricane hazard modeling: The past, present, and future. Journal of Wind Engineering and Industrial Aerodynamics, 97(7), 392-405.

Vickery, P. J., Skerlj, P., Steckley, A., \& Twisdale, L. (2000a). Hurricane wind field model for use in hurricane simulations. Journal of Structural Engineering, 126(10), 12031221.

Vickery, P. J., Skerlj, P., \& Twisdale, L. (2000b). Simulation of Hurricane Risk in the U.S. Using Empirical Track Model. Journal of Structural Engineering, 126(10), 12221237.

Vickery, P. J., \& Twisdale, L. A. (1995a). Prediction of hurricane wind speeds in the United States. Journal of Structural Engineering, 121(11), 1691-1699.

Vickery, P. J., \& Twisdale, L. A. (1995b). Wind-field and filling models for hurricane wind-speed predictions. Journal of Structural Engineering, 121(11), 1700-1709.

Vickery, P. J., Wadhera, D., Twisdale Jr, L. A., \& Lavelle, F. M. (2009b). US hurricane wind speed risk and uncertainty. Journal of Structural Engineering, 135(3), 301320.

Viswanadha Raju, G., \& Bijwe, P. (2008). An efficient algorithm for minimum loss reconfiguration of distribution system based on sensitivity and heuristics. Power Systems, IEEE Transactions on, 23(3), 1280-1287.

Volkanovski, A., Čepin, M., \& Mavko, B. (2009). Application of the fault tree analysis for assessment of power system reliability. Reliability Engineering \& System Safety, 94(6), 1116-1127.

Walsh, J., D. , Wuebbles, K., Hayhoe, J., Kossin, K., Kunkel, G., Stephens, P., . . R. R. Somerville. (2014). Our Changing Climate. Climate Change Impacts in the United States: The Third National Climate Assessment. In J. M. Melillo, Terese (T.C.) Richmond \& G. W. Yohe (Eds.), (pp. 19-67): U.S. Global Change Research Program.

Wang, Y., \& Rosowsky, D. V. (2012). Joint distribution model for prediction of hurricane wind speed and size. Structural Safety, 35(0), 40-51. 
WBCSD. (2014). Building a Resilient Power Sector: World Business Council For Sustainable Development - Electric Utilities Project.

Weichselgartner, J. (2001). Disaster mitigation: the concept of vulnerability revisited. Disaster Prevention and Management: An International Journal, 10(2), 85-95. doi: $10.1108 / 09653560110388609$

Wen, Y., \& Kang, Y. (2001). Minimum Building Life-Cycle Cost Design Criteria. II: Applications. Journal of Structural Engineering, 127(3), 338-346. doi: doi:10.1061/(ASCE)0733-9445(2001)127:3(338)

Winkler, J., Dueñas-Osorio, L., Stein, R., \& Subramanian, D. (2010). Performance assessment of topologically diverse power systems subjected to hurricane events. Reliability Engineering \& System Safety, 95(4), 323-336.

Wolfe, R., \& Moody, R. (1997). Standard specifications for wood poles. Paper presented at the Utility Pole Structures Conf and Trade Show; Nov. 6-7, 1997; Reno/Sparks, Nevada.

Xu, L., \& Brown, R. E. (2008a). A hurricane simulation method for Florida utility damage and risk assessment. Paper presented at the Power and Energy Society General Meeting-Conversion and Delivery of Electrical Energy in the 21st Century, 2008 IEEE.

Xu, L., \& Brown, R. E. (2008b). Undergrounding assessment Phase 3 Report: Ex Ante Cost and Benefit Modeling. Raleigh: Quanta Technology.

Yeo, D., Lin, N., \& Simiu, E. (2014). Estimation of Hurricane Wind Speed Probabilities: Application to New York City and Other Coastal Locations. Journal of Structural Engineering, 140(6), 04014017. doi: doi:10.1061/(ASCE)ST.1943-541X.0000892

Zamuda, C., Mignone, B., Bilello, D., Hallett, K., Lee, C., Macknick, J., . . Steinberg, D. (2013). US Energy Sector Vulnerabilities to Climate Change and Extreme Weather: DTIC Document. 


\section{Appendix A: Document showing that Fig. 2.2 is in the public domain}




\section{U.S. Geological Survey}

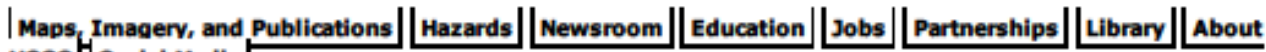
USGS Social Media

\section{Information Policies and Instructions}

The USGS serves the Nation as an independent fact-finding agency that collects, monitors, analyzes, and provides scientific understanding about natural resource and natural hazard conditions, issues, and problems. The value of the USGS to the Nation rests on its ability to carry out studies on a national scale and to sustain long-term monitoring and assessment of natural resources and hazards. Because it has no regulatory or management mandate, the USGS provides impartial science that serves the needs of our changing world. It is the policy of the USGS to conduct its activities and to make the results of its scientific investigations available in a manner that will best serve the whole public, rather than the interest or benefit of any special group, corporation, or individual. The reputation of USGS science for excellence and objectivity is our most important asset. It brings authority to our data and findings and creates long-term credibility.

\section{Information Quality}

In Section 515(a) of the Treasury and General Government Appropriations Act for Fiscal Year 2001, Congress directed the Office of Management and Budget (OMB) to issue governmentwide guidelines that "provide policy and procedural guidance to Federal agencies for ensuring and maximizing the quality, objectivity, utility, and integrity of information (including statistical information) disseminated by Federal agencies."

The USGS guidelines and complaint process will apply to all information disseminated on or after October 1, 2002. Information disseminated prior to October 1, 2002, but not archived and still being used in a decisionmaking process is not exempt from these guidelines. Information records disseminated from studies completed and archived before October 1, 2002, are exempt from the guidelines.

Contact - If you have questions on USGS data and information accuracy, contact:

USGS Information
Mail Stop 807
National Center
USGS
Reston, VA 20192

\section{Public Release of Information}

The USGS, as the science arm of the Department of the Interior and the earth and natural science agency for the Nation, has an ongoing obligation to keep the broadest spectrum of the public advised and engaged in its scientific research, investigations, and ongoing information releases. The USGS Home Paoe, its links to science information of the various programs and activities of the USGS, the resources of the USGS Newsroom, and the USGS search function all provide prompt and easy public access to the results of USGS scientific research and investigations.

Section 207 (f) (2) of the E-Government Act of 2002 requires Government agencies to develop an inventory of information to be published on their Web site. The purpose of Section 207 ( $f$ ) (2) is to improve the methods by which Government information, including information on the Internet, is organized, preserved, and made accessible to the public. More information on this requirement can be found on the National Archives and Records Administration Web site at httro//www_archives,oov/about us/basic_laws and_authorities/eoov act section 207.html. 
Policy on Release of Information - As an integral part of its mission, the USGS has a continuing responsibility to keep the public informed of its various programs, activities, and research. It is the policy of the USGS to conduct its activities and to make the results of its scientific investigations available in a manner that will best serve the whole public, rather than the interest or benefit of any special group, corporation, or individual. The Internet affords the USGS a mechanism to achieve that broad distribution, impartially and simultaneously, in accordance with its mission and information dissemination policy.

Schedule and Priorities for Release of Information - The diversity of scientific expertise enables the USGS to carry out large-scale, multidisciplinary investigations that build the base of knowledge about the Earth. In turn, decisionmakers at all levels of government-and citizens in all walks of life-have the information tools they need to address pressing societal issues. As such, there is no set schedule on which the USGS releases its information; rather, the readiness of the science for release determines the schedule. Priority of release is consistent with the Department of the Interior Schedule of Content.

News releases are issued and information is posted on the USGS Home Pane whenever a significant earthquake or other natural disaster occurs, a major personnel appointment is announced, budget information is available, or a science program has results to announce.

The USGS is a scientific publisher and regularly and routinely presents the results of its scientific research and investigations in printed and/or online format in publication series. Bibliographic citations and information on accessing and obtaining USGS information products are available in the USGS Publications Warehouse.

Contact - If you have questions concerning release of USGS information, contact the USGS Web.

\section{Copyrights and Credits}

USGS-authored or produced data and information are considered to be in the U.S. public domain. While the content of most USGS Web pages is in the U.S. public domain, not al information, illustrations, or photographs on our site are. Some non USGS photographs, images, and/or graphics that appear on USGS Web sites are used by the USGS with permission from the copyright holder. These materials are generally marked as being copyrighted. To use these copyrighted materials, you must obtain permission from the copyright holder under the copyright law.

When using information from USGS information products, publications, or Web sites, we ask that proper credit be given. Credit can be provided by including a citation such as the following:

Credit: U.S. Geological Survey

Department of the Interior/USGS

U.S. Geological Survey/photo by Jane Doe (if the photographer/artist is known)

Additional information on Acknowledoing or Crediting USGS as Information Source is available.

Policy - For relevant USGS policy on copyrights and trademarks, refer to the Survey Manual chapter entitled, "Use of Copvriahted Material_in USGS Information_Products."

Contact - If you have questions concerning the use of USGS information, contact the USGS Web.

\section{USGS Visual Identity System}

To reinforce visually who we are and what we do, the USGS uses a visual identity system that provides planning and design solutions for USGS information products. A key component of the visual identity system is the USGS identifier. This identifier is trademarked and must be used in accordance with the rules outlined at Visual_dentity Svstem. Information on who may use the USGS identifier and how to obtain a copy of it is available at Use-of the-Trademarked USGS Identifier by Non-USGS Oroanizations. 
Policy - For relevant USGS policy on the Visual Identity System, refer to the Survey Manual chapter entitled, "USGS Visual_identity System."

Contact - If you have questions on the USGS Visual Identity System, contact the USGS Visual Identity System Helo Desk.

\section{Liability}

Neither the U.S. Government, the Department of the Interior, nor the USGS, nor any of their employees, contractors, or subcontractors, make any warranty, express or implied, nor assume any legal liability or responsibility for the accuracy, completeness, or usefulness of any information, apparatus, product, or process disclosed, nor represent that its use would not infringe on privately owned rights.

Links to non-USGS sites - Links and pointers to non-USGS sites are provided for information only and do not constitute endorsement by the USGS, U.S. Department of the Interior, or U.S. Government, of the referenced organizations, their suitability, content, products, or services, whether they are governmental, educational, or commercial. Some of the documents on this server may contain live references (or pointers) to information created and maintained by other organizations. Please note that the USGS does not control and cannot guarantee the relevance, timeliness, or accuracy of these outside materials.

Non-endorsement of non-USGS products and services - Hypertext links and other references to non-USGS products and services are provided for information only and do not constitute endorsement or warranty by the USGS, U.S. Department of the Interior, or U.S. Government, as to their suitability, content, usefulness, functioning, completeness, or accuracy.

Public domain software - Links are provided to public-domain software developed by or for the USGS. Although these programs have been used by the USGS, no warranty is made by the USGS as to the accuracy and functioning of the programs and related program material. Distribution of these materials also does not constitute any warranty, and no responsibility is assumed by the USGS in connection with any distribution activity.

Contact - If you have general questions about USGS Web sites, contact the USGS Web.

U.S. Department of the Interior I U.S. Geological Survey

URL: http://www.usgs.gov/laws/info_policies.html

Page Contact Information: Ask USGS

Page Last Modified: 14-Dec-2012@11:47 
Appendix B: Permission to publish Chapter 4 
This Agreement between Abdullahi M Salman ("You") and Elsevier ("Elsevier") consists of your license details and the terms and conditions provided by Elsevier and Copyright Clearance Center.

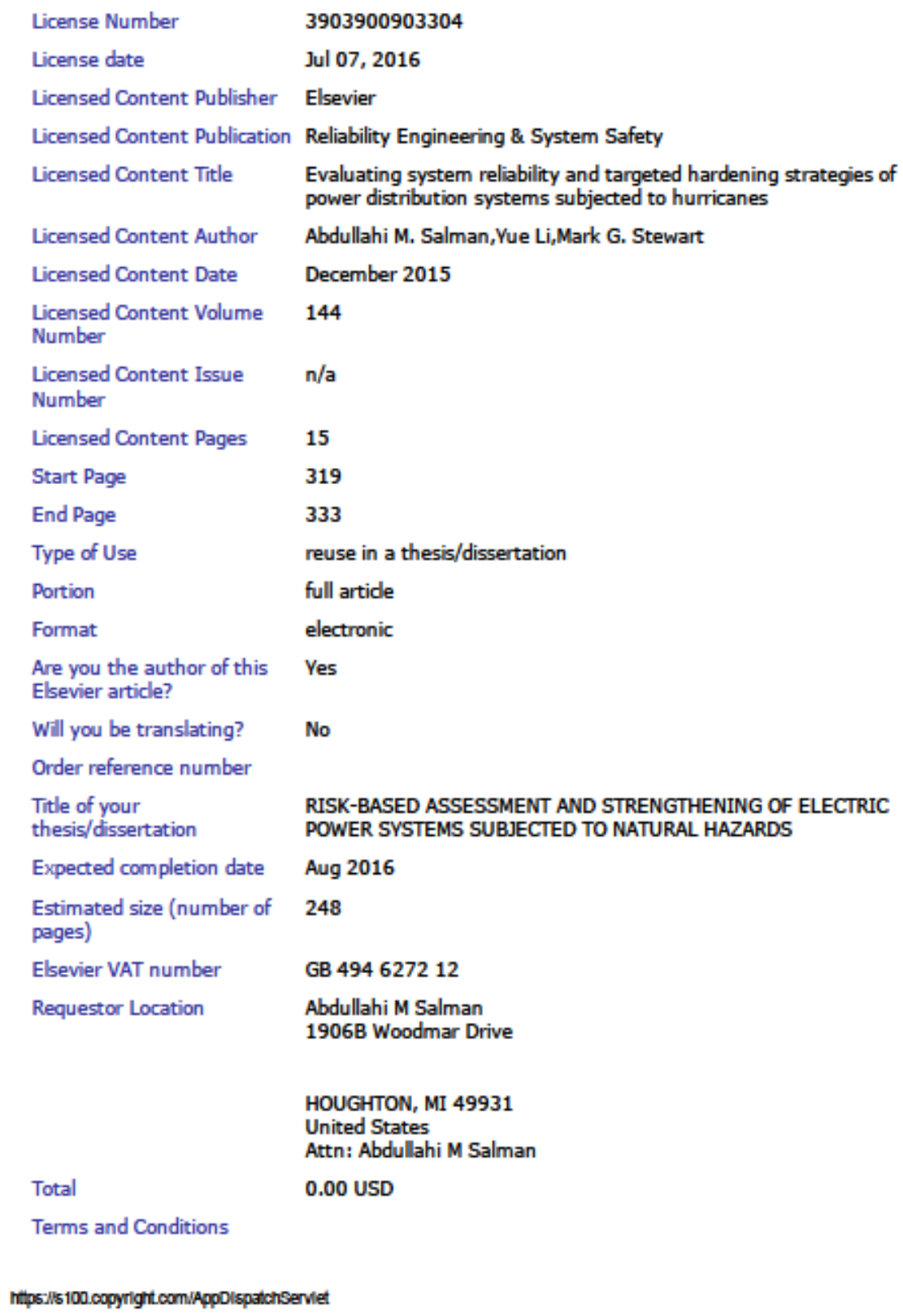




\section{INTRODUCTION}

1. The publisher for this copyrighted material is Elsevier. By clicking "accept" in connection with completing this licensing transaction, you agree that the following terms and conditions apply to this transaction (along with the Billing and Payment terms and conditions established by Copyright Clearance Center, Inc. ("CCC"), at the time that you opened your Rightslink account and that are available at any time at htto://myaccount.copyright.com).

\section{GENERAL TERMS}

2. Elsevier hereby grants you permission to reproduce the aforementioned material subject to the terms and conditions indicated.

3. Acknowledgement: If any part of the material to be used (for example, figures) has appeared in our publication with credit or acknowledgement to another source, permission must also be sought from that source. If such permission is not obtained then that material may not be included in your publication/copies. Suitable acknowledgement to the source must be made, either as a footnote or in a reference list at the end of your publication, as follows:

"Reprinted from Publication title, Vol /edition number, Author(s), Title of article / title of chapter, Pages No., Copyright (Year), with permission from Elsevier [OR APPLICABLE SOCIETY COPYRIGHT OWNER]." Also Lancet special credit - "Reprinted from The Lancet, Vol. number, Author(s), Title of article, Pages No., Copyright (Year), with permission from Elsevier."

4. Reproduction of this material is confined to the purpose and/or media for which permission is hereby given.

5. Altering/Modifying Material: Not Permitted. However figures and illustrations may be altered/adapted minimally to serve your work. Any other abbreviations, additions, deletions and/or any other alterations shall be made only with prior written authorization of Elsevier Ltd. (Please contact Elsevier at permissions@elsevier.com)

6. If the permission fee for the requested use of our material is waived in this instance, please be advised that your future requests for Elsevier materials may attract a fee. 7. Reservation of Rights: Publisher reserves all rights not specifically granted in the combination of (i) the license details provided by you and accepted in the course of this licensing transaction, (ii) these terms and conditions and (iii) CCC's Billing and Payment terms and conditions.

8. License Contingent Upon Payment: While you may exercise the rights licensed immediately upon issuance of the license at the end of the licensing process for the transaction, provided that you have disclosed complete and accurate details of your proposed use, no license is finally effective unless and until full payment is received from you (either by publisher or by $\mathrm{CCC}$ ) as provided in CCC's Billing and Payment terms and conditions. If full payment is not received on a timely basis, then any license preliminarily granted shall be deemed automatically revoked and shall be void as if never granted. Further, in the event that you breach any of these terms and conditions or any of CCC's Billing and Payment terms and conditions, the license is automatically revoked and shall be void as if never granted. Use of materials as described in a revoked license, as well as any use of the materials beyond the scope of an unrevoked license, may constitute copyright infringement and publisher reserves the right to take any and all action to protect its copyright in the materials.

9. Warranties: Publisher makes no representations or warranties with respect to the licensed material.

10. Indemnity: You hereby indemnify and agree to hold harmless publisher and $\mathrm{CCC}$, and their respective officers, directors, employees and agents, from and against any and all claims arising out of your use of the licensed material other than as specifically authorized pursuant to this license.

11. No Transfer of License: This license is personal to you and may not be sublicensed, assigned, or transferred by you to any other person without publisher's written permission. 12. No Amendment Except in Writing: This license may not be amended except in a writing 
signed by both parties (or, in the case of publisher, by CCC on publisher's behalf). 13. Objection to Contrary Terms: Publisher hereby objects to any terms contained in any purchase order, acknowledgment, check endorsement or other writing prepared by you, which terms are inconsistent with these terms and conditions or CCC's Billing and Payment terms and conditions. These terms and conditions, together with CCC's Billing and Payment terms and conditions (which are incorporated herein), comprise the entire agreement between you and publisher (and CCC) concerning this licensing transaction. In the event of any conflict between your obligations established by these terms and conditions and those established by $\mathrm{CCC}$ 's Billing and Payment terms and conditions, these terms and conditions shall control.

14. Revocation: Elsevier or Copyright Clearance Center may deny the permissions described in this License at their sole discretion, for any reason or no reason, with a full refund payable to you. Notice of such denial will be made using the contact information provided by you. Failure to receive such notice will not alter or invalidate the denial. In no event will Elsevier or Copyright Clearance Center be responsible or liable for any costs, expenses or damage incurred by you as a result of a denial of your permission request, other than a refind of the amount(s) paid by you to Elsevier and/or Copyright Clearance Center for denied permissions.

\section{LIMITED LICENSE}

The following terms and conditions apply only to specific license types:

15. Translation: This permission is granted for non-exclusive world English rights only unless your license was granted for translation rights. If you licensed translation rights you may only translate this content into the languages you requested. A professional translator must perform all translations and reproduce the content word for word preserving the integrity of the article.

16. Posting licensed content on any Website: The following terms and conditions apply as follows: Licensing material from an Elsevier journal: All content posted to the web site must maintain the copyright information line on the bottom of each image; A hyper-text must be included to the Homepage of the journal from which you are licensing at $\mathrm{http}$ ///www.sciencedirect.com/science/ioumal/sxxxx or the Elsevier homepage for books at http://wwwelsevier.com; Central Storage: This license does not include permission for a scanned version of the material to be stored in a central repository such as that provided by Heron/XanEdu.

Licensing material from an Elsevier book: A hyper-text link must be included to the Elsevier homepage at http://www elsevier.com. All content posted to the web site must maintain the copyright information line on the bottom of each image.

Posting licensed content on Electronic reserve: In addition to the above the following clauses are applicable: The web site must be password-protected and made available only to bona fide students registered on a relevant course. This permission is granted for 1 year only. You may obtain a new license for future website posting.

17. For journal authors: the following clauses are applicable in addition to the above: Preprints:

A preprint is an author's own write-up of research results and analysis, it has not been peerreviewed, nor has it had any other value added to it by a publisher (such as formatting, copyright, technical enhancement etc.).

Authors can share their preprints anywhere at any time. Preprints should not be added to or enhanced in any way in order to appear more like, or to substitute for, the final versions of articles however authors can update their preprints on arXiv or RePEc with their Accepted Author Manuscript (see below).

If accepted for publication, we encourage authors to link from the preprint to their formal publication via its DOI. Millions of researchers have access to the formal publications on ScienceDirect, and so links will help users to find, access, cite and use the best available version. Please note that Cell Press, The Lancet and some society-owned have different 
preprint policies. Information on these policies is available on the journal homepage. Accepted Author Manuscripts: An accepted author manuscript is the manuscript of an article that has been accepted for publication and which typically includes authorincorporated changes suggested during submission, peer review and editor-author communications.

Authors can share their accepted author manuscript:

- immediately

- via their non-commercial person homepage or blog

- by updating a preprint in arXiv or RePEc with the accepted manuscript

- via their research institute or institutional repository for internal institutional uses or as part of an invitation-only research collaboration work-group

- directly by providing copies to their students or to research collaborators for their personal use

- for private scholarly sharing as part of an invitation-only work group on commercial sites with which Elsevier has an agreement

- after the embargo period

- via non-commercial hosting platforms such as their institutional repository

- via commercial sites with which Elsevier has an agreement

In all cases accepted manuscripts should:

- link to the formal publication via its DOI

- bear a CC-BY-NC-ND license - this is easy to do

- if aggregated with other manuscripts, for example in a repository or other site, be shared in alignment with our hosting policy not be added to or enhanced in any way to appear more like, or to substitute for, the published journal article.

Published journal article (JPA): A published journal article (PJA) is the definitive final record of published research that appears or will appear in the journal and embodies all value-adding publishing activities including peer review co-ordination, copy-editing, formatting, (if relevant) pagination and online enrichment.

Policies for sharing publishing journal articles differ for subscription and gold open access articles:

Subscription Articles: If you are an author, please share a link to your article rather than the full-text. Millions of researchers have access to the formal publications on ScienceDirect, and so links will help your users to find, access, cite, and use the best available version. Theses and dissertations which contain embedded PJAs as part of the formal submission can be posted publicly by the awarding institution with DOI links back to the formal publications on ScienceDirect.

If you are affiliated with a library that subscribes to ScienceDirect you have additional private sharing rights for others' research accessed under that agreement. This includes use for classroom teaching and internal training at the institution (including use in course packs and courseware programs), and inclusion of the article for grant funding purposes.

Gold Open Access Articles: May be shared according to the author-selected end-user license and should contain a CrossMark logo, the end user license, and a DOI link to the formal publication on ScienceDirect.

Please refer to Elsevier's posting policy for further information.

18. For book authors the following clauses are applicable in addition to the above: Authors are permitted to place a brief summary of their work online only. You are not allowed to download and post the published electronic version of your chapter, nor may you scan the printed edition to create an electronic version. Posting to a repository: Authors are permitted to post a summary of their chapter only in their institution's repository.

19. Thesis/Dissertation: If your license is for use in a thesis/dissertation your thesis may be 
submitted to your institution in either print or electronic form. Should your thesis be published commercially, please reapply for permission. These requirements include permission for the Library and Archives of Canada to supply single copies, on demand, of the complete thesis and include permission for Proquest/UMI to supply single copies, on demand, of the complete thesis. Should your thesis be published commercially, please reapply for permission. Theses and dissertations which contain embedded PJAs as part of the formal submission can be posted publicly by the awarding institution with DOI links back to the formal publications on ScienceDirect.

\section{Elsevier Open Access Terms and Conditions}

You can publish open access with Elsevier in hundreds of open access journals or in nearly 2000 established subscription journals that support open access publishing. Permitted third party re-use of these open access articles is defined by the author's choice of Creative Commons user license. See our open access license policy for more information.

Terms \& Conditions applicable to all Open Access articles published with Elsevier: Any reuse of the article must not represent the author as endorsing the adaptation of the article nor should the article be modified in such a way as to damage the author's honour or reputation. If any changes have been made, such changes must be clearly indicated. The author(s) must be appropriately credited and we ask that you include the end user license and a DOI link to the formal publication on ScienceDirect.

If any part of the material to be used (for example, figures) has appeared in our publication with credit or acknowledgement to another source it is the responsibility of the user to ensure their reuse complies with the terms and conditions determined by the rights holder. Additional Terms \& Conditions applicable to each Creative Commons user license: CC BY: The CC-BY license allows users to copy, to create extracts, abstracts and new works from the Article, to alter and revise the Article and to make commercial use of the Article (including reuse and/or resale of the Article by commercial entities), provided the user gives appropriate credit (with a link to the formal publication through the relevant DOI), provides a link to the license, indicates if changes were made and the licensor is not represented as endorsing the use made of the work. The full details of the license are available at http://creativecommons.org/licenses/by/4.0.

CC BY NC SA: The CC BY-NC-SA license allows users to copy, to create extracts, abstracts and new works from the Article, to alter and revise the Article, provided this is not done for commercial purposes, and that the user gives appropriate credit (with a link to the formal publication through the relevant DOI), provides a link to the license, indicates if changes were made and the licensor is not represented as endorsing the use made of the work. Further, any new works must be made available on the same conditions. The full details of the license are available at $h \mathrm{hth} / / /$ creativecommons.org/licenses/bv-nc-sa/4.0. CC BY NC ND: The CC BY-NC-ND license allows users to copy and distribute the Article, provided this is not done for commercial purposes and further does not permit distribution of the Article if it is changed or edited in any way, and provided the user gives appropriate credit (with a link to the formal publication through the relevant DOI), provides a link to the license, and that the licensor is not represented as endorsing the use made of the work. The full details of the license are available at http://creativecommons_org/licenses/by-nc-nd/4.0. Any commercial reuse of Open Access articles published with a CC BY NC SÁ or CC BY NC ND license requires permission from Elsevier and will be subject to a fee.

Commercial reuse includes:

- Associating advertising with the full text of the Article

- Charging fees for document delivery or access

- Article aggregation

- Systematic distribution via e-mail lists or share buttons

Posting or linking by commercial companies for use by customers of those companies. 


\section{Other Conditions:}

v1.8

Questions? customercare@copyriahtccom or +1-855-239-3415 (toll free in the US) or +1-978-646-2777. 
Appendix C: Permission to use Fig. $4.2 \& 4.3$ 


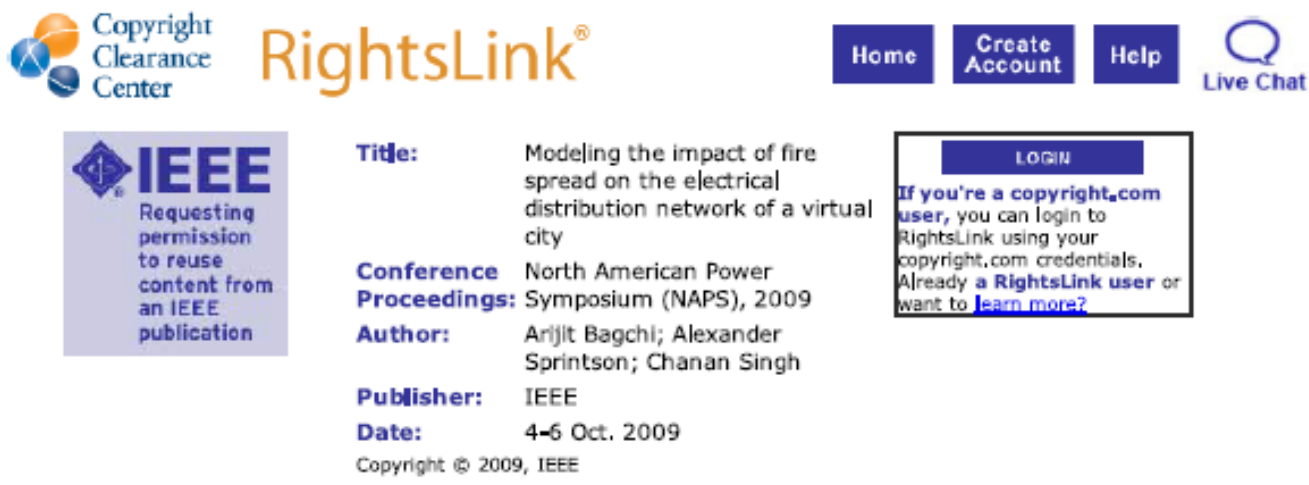

Thesis / Dissertation Reuse

The IEEE does not require individuals working on a thesis to obtain a formal reuse license, however, you may print out this statement to be used as a permission grant:

Requirements to be followed when using any portion (e.g., figure, graph, table, or textual material) of an IEEE copyrighted paper in a thesis:

1) In the case of textual material (e.g., using short quotes or referring to the work within these papers) users must give full credit to the original source (author, paper, publication) followed by the IEEE copyright line 2011 IEEE.

2) In the case of illustrations or tabular material, we require that the copyright line [Year of origina publication] IEEE appear prominently with each reprinted figure and/or table,

3) If a substantial portion of the original paper is to be used, and if you are not the senior author, also obtain the senior author approval.

Requirements to be followed when using an entire IEEE copyrighted paper in a thesis:

1) The following IEEE copyright/ credit notice should be placed prominently in the references: [year of original publication] IEEE. Reprinted, with permission, from [author names, paper title, IEEE publication title, and month/year of publication]

2) Only the accepted version of an IEEE copyrighted paper can be used when posting the paper or your thesis on-line,

3) In placing the thesis on the author's university website, please display the following message in a prominent place on the website: In reference to IEEE copyrighted material which is used with permission in this thesis, the IEEE does not endorse any of [university/educational entity's name goes here]'s products or services, Internal or personal use of this material is permitted, If interested in

reprinting/republishing IEEE copyrighted material for advertising or promotional purposes or for creating new collective works for resale or redistribution, please go to

htto://www deeenora/oublications_standards/oublications/riahts/riahts_link.html to learn how to obtain a License from RightsLink,

If applicable, University Microfilms and/or ProQuest Library, or the Archives of Canada may supply single copies of the dissertation.

\section{BACK CLOSE WINDOW}

Copyright (2) 2016 Copsriaht Clearance Center. Inc. All Rights Reserved. Privacy statement. Ierms and Conditions.

Comments? We would like to hear from you, E-mail us at oustomercareapconvrioht.com 
Appendix D: Permission to publish Chapter 5 


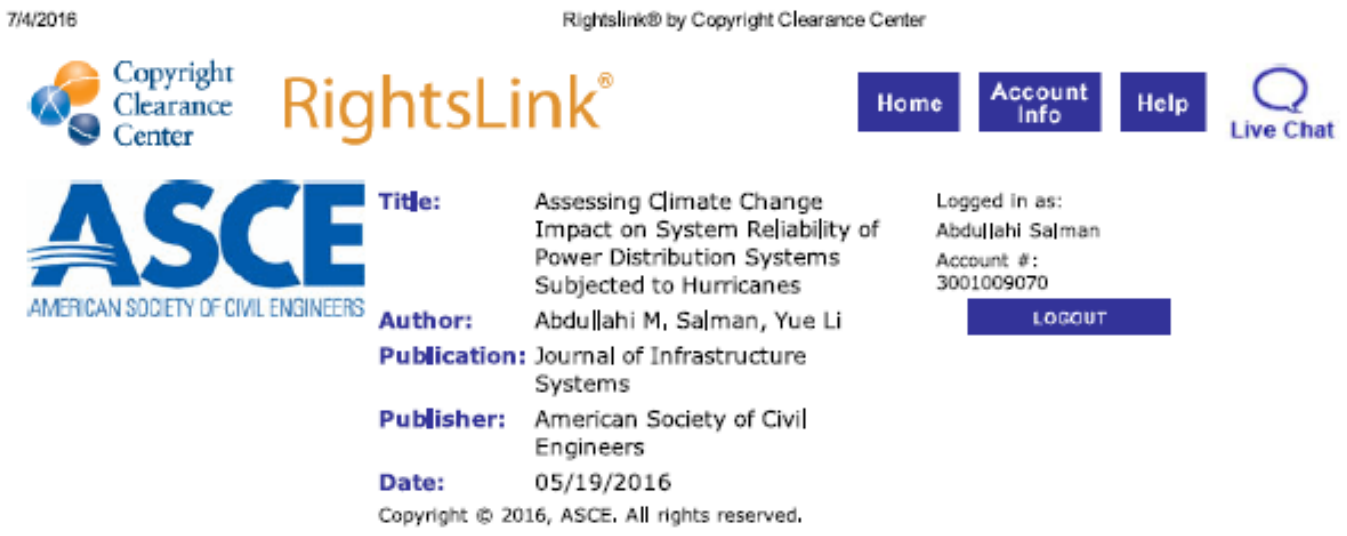

Permissions Request

As an author of an ASCE journal artide, you are permitted to reuse the accepted manuscript version of your article for your thesis or dissertation.

\section{BACK CLOSE WINDOW}

Copyright if 2016 Ceovriaht Glearance Center, Inc. All Rights Reserved. Privacy statement. Ierms and Conditions, Comments? We would like to hear from you, E-mail us at customercarefincopyricht.com 
Appendix E: Permission to use base map of Figure 6.2 


\section{Copyright and License}

OpenStreetMap is open data, licensed under the Open Data Commons Open Database License (ODbL) by the OpenStreetMap Foundation (OSMF).

You are free to copy, distribute, transmit and adapt our data, as long as you credit OpenStreetMap and its contributors. If you alter or build upon our data, you may distribute the result only under the same licence. The full legal code explains your rights and responsibilities.

The cartography in our map tiles, and our documentation, are licensed under the Creative Commons AttributionShareAlike 2.0 license (CC BY-SA).

\section{How to credit OpenStreetMap}

We require that you use the credit "(8) OpenStreetMap contributors".

You must also make it clear that the data is available under the Open Database License, and if using our map tiles, that the cartography is licensed as CC BY-SA. You may do this by linking to this copyright page.

Alternatively, and as a requirement if you are distributing OSM in a data form, you can name and link directly to the license(s). In media where links are not possible (e.g. printed works), we suggest you direct your readers to openstreetmap.org (perhaps by expanding 'OpenStreetMap' to this full address), to opendatacommons.org, and if relevant, to creativecommons.org.

For a browsable electronic map, the credit should appear in the corner of the map. For example:

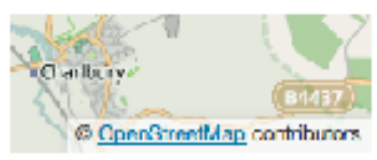

\section{Finding out more}

Read more about using our data, and how to credit us, at the OSMF Licence page and the community Legal EAO

Although OpenStreetMap is open data, we cannot provide a free-of-charge map API for third-party developers. See our API Usage Policy. Tile Usage Policy and Nominatim Usage Policy.

\section{Our contributors}

Our contributors are thousands of individuals. We also include openly-licensed data from national mapping agencies and other sources, among them:

- Austria: Contains data from Stadt Wien (under $\mathrm{CC} B \mathrm{~B}$ ), Land Vorarlberg and Land Tirol (under CC BY AT with amendments).

- Canada: Contains data from GeoBase@, GeoGratis (@ Department of Natural Resources Canada), CanVec (8 Department of Natural Resources Canada), and StatCan (Geography Division, Statistics Canada).

- Finland: Contains data from the National Land Survey of Finland's Topographic Database and other datasets, under the NLSELLicense.

- France: Contains data sourced from Direction Générale des Impôts. 
- Netherlands: Contains @ AND data, 2007 (wwwwand.com)

- New Zealand: Contains data sourced from Land Information New Zealand. Crown Copyright reserved.

- Slovenia: Contains data from the Surveying andMapping Authority and Ministry of Aqriculture. Forestry and Food (public information of Slovenia).

- South Africa: Contains data sourced from ChiefDirectorate:National Geo-Spatial Information, State copyright reserved.

- United Kingdom: Contains Ordnance Survey data @ Crown copyright and database right 2010-12.

For further details of these, and other sources that have been used to help improve OpenStreetMap, please see the Contributors page on the OpenStreetMap Wiki.

Inclusion of data in OpenStreetMap does not imply that the original data provider endorses OpenStreetMap, provides any warranty, or accepts any liability.

\section{Copyright infringement}

OSM contributors are reminded never to add data from any copyrighted sources (e.g. Google Maps or printed maps) without explicit permission from the copyright holders.

If you believe that copyrighted material has been inappropriately added to the OpenStreetMap database or this site, please refer to our takedown procedure or file directly at our on-line filing page.

\section{Trademarks}

OpenStreetMap, the magnifying glass logo and State of the Map are registered trademarks of the OpenStreetMap Foundation. If you have questions about your use of the marks, please send your questions to the Licence Working Group. 